SLAC-R-770

\title{
D-Branes in Curved Space
}

\author{
John Austen McGreevy
}




\title{
D-BRANES IN CURVED SPACE
}

\author{
A DISSERTATION \\ SUBMITTED TO THE DEPARTMENT OF PHYSICS \\ AND THE COMMITTEE ON GRADUATE STUDIES \\ OF STANFORD UNIVERSITY \\ IN PARTIAL FULFILLMENT OF THE REQUIREMENTS \\ FOR THE DEGREE OF \\ DOCTOR OF PHILOSOPHY
}

John McGreevy

June 2002 


\title{
D-BRANES IN CURVED SPACE
}

\author{
John McGreevy, Ph.D. \\ Stanford University, 2002 \\ Advisor: Shamit Kachru
}

This thesis is a study of D-branes in string compactifications. In this context, Dbranes are relevant as an important component of the nonperturbative spectrum, as an incisive probe of these backgrounds, and as a natural stringy tool for localizing gauge interactions.

In the first part of the thesis, we discuss half-BPS D-branes in compactifications of type II string theory on Calabi-Yau threefolds. The results we describe for these objects are pertinent both in their role as stringy brane-worlds, and in their role as solitonic objects. In particular, we determine couplings of these branes to the moduli determining the closedstring geometry, both perturbatively and non-perturbatively in the worldsheet expansion. We provide a local model for transitions in moduli space where the BPS spectrum jumps, and discuss the extension of mirror symmetry between Calabi-Yau manifolds to the case when D-branes are present.

The next section is an interlude which provides some applications of D-branes to other curved backgrounds of string theory. In particular, we discuss a surprising phenomenon in which fundamental strings moving through background Ramond-Ramond fields dissolve into large spherical D3-branes. This mechanism is used to explain a previously-mysterious fact discovered via the $A d S$-CFT correspondence. Next, we make a connection between type IIA string vacua of the type discussed in the first section and M-theory compactifications on manifolds of $G_{2}$ holonomy. Finally we discuss constructions of string vacua which do not have large radius limits.

In the final part of the thesis, we develop techniques for studying the worldsheets of open strings ending on the curved D-branes studied in the first section. More precisely, we formulate a large class of massive two-dimensional gauge theories coupled to boundary matter, which flow in the infrared to the relevant boundary conformal field theories. Along with many other applications, these techniques are used to describe worldvolume theories of pointlike D-probes of various Calabi-Yau threefolds.

Approved for publication:

By:

For the Department of Physics 


\section{Acknowledgements}

I would like to express my gratitude to the members of the string theory groups

at Berkeley and Stanford for teaching me physics and for providing a stimulating

environment in which to work. I am very grateful to all of my collaborators for

sharing their time and ideas with me, and for not minding that I include our work

in this document. Thank you to my advisor, Shamit Kachru, for guidance, per-

spective and inspiration. Thank you to Simeon Hellerman for his patience and

fortitude. Thank you to Michael Schulz for a million favors and amazing attention

to administrative detail. Finally, I must thank my parents for decades of support

and encouragement, and Kirthi Reddy for all kinds of things.

I acknowledge the support of an NDSEG Graduate Fellowship and National

Science Foundation grant PHY-0097915. 


\section{Contents}

1. Friendly Introduction . . . . . . . . . . . . . . . . . . . . 1

Part I . . . . . . . . . . . . . . 7

2. Supersymmetric Three-cycles and (Super)symmetry breaking . . 7

2.1. Introduction . . . . . . . . . . . . . . . . . . . . 7

2.2. Splitting Supersymmetric Cycles . . . . . . . . . . . . . . . . . 8

2.2.1. Definitions . . . . . . . . . . . . . . . . . . . . . . . 8

2.2.2. Transitions . . . . . . . . . . . . . . . . . . . . . . . . . 9

2.3. Formerly BPS States in IIB String Theory . . . . . . . . . . . . . 11

2.4. D6-Branes and the Fayet Model . . . . . . . . . . . . . . . . . 13

2.5. Discussion . . . . . . . . . . . . . . . . . . . . . . . . . . . 14

3. Open String Instantons and Superpotentials _. . . . . . . . . 16

3.1. Introduction . . . . . . . . . . . . . . . . . . . . . . . . . . 16

3.2. Classical geometry of D-branes on $C Y_{3}$. . . . . . . . . . . . . . . 19

3.2.1. A-type branes . . . . . . . . . . . . . . . . . . . . . . . 19

3.2.2. B-type branes . . . . . . . . . . . . . . . . . . . . . . 23

3.3. Disc instantons . . . . . . . . . . . . . . . . . . . . . . . . 29

3.4. Discussion . . . . . . . . . . . . . . . . . . . . . . . . . . . 35

4. Mirror Symmetry for Open Strings _ . . . . . . . . . . . . . . 37

4.1. Introduction . . . . . . . . . . . . . . . . . . . . . . . . . . 37

4.2. Superpotentials from D-branes . . . . . . . . . . . . . . . . . . 39

4.2.1. A-type and B-type branes . . . . . . . . . . . . . . . . . . 39

4.2.2. Superpotentials for B-type branes . . . . . . . . . . . . . . . 40

4.2.3. Qualitative features of superpotentials for A-type branes . . . . . 45

4.3. Constructing the mirror three-cycles . . . . . . . . . . . . . . . 48

4.3.1. Strategy for identifying mirror cycles . . . . . . . . . . . . . 48

4.3.2. Example III . . . . . . . . . . . . . . . . . . . . . . . . 50

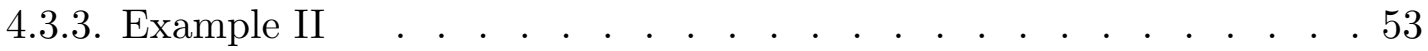

4.3.4. Example I . . . . . . . . . . . . . . . . . . . . . . . . . 55

4.4. Mirror symmetry and the superpotential . . . . . . . . . . . 56

4.4.1. The superpotential near the toric locus . . . . . . . . . . . . 57

4.4.2. The mirror map . . . . . . . . . . . . . . . . . . . . . . . 59

4.5. Discussion . . . . . . . . . . . . . . . . . . . . . . . . . . . 64

Appendix 4.A. Some Details Concerning Example I . . . . . . . . . . 66

Part II . . . . . . . . . . . . . . . . 69

5. Invasion of the Giant Gravitons from Anti de Sitter Space . . . 69

5.1. Introduction . . . . . . . . . . . . . . . . . . . . . . . . 70

5.2. Dipoles in Magnetic Fields . . . . . . . . . . . . . . . . . 71

5.3. $A d S \times S$. . . . . . . . . . . . . . . . . . . . . . . . . . . 73

5.3.1. $A d S_{7} \times S^{4}$. . . . . . . . . . . . . . . . . . . . . . . . . 73

5.3.2. $A d S_{5} \times S^{5}$. . . . . . . . . . . . . . . . . . . . . . . . . 79

5.3.3. $A d S_{4} \times S^{7}$. . . . . . . . . . . . . . . . . . . . . . 81

5.3.4. Remarks about $A d S_{3}$. . . . . . . . . . . . . . . . . . . . 82

5.4. Conclusions . . . . . . . . . . . . . . . . . . . . . . . . . . 82 
5.5. Acknowledgments . . . . . . . . . . . . . . . . . . . . . . . 83

6. M-theory on Manifolds of $G_{2}$ Holonomy . . . . . . . . . . . . . 84

6.1. Introduction . . . . . . . . . . . . . . . . . . . . . . . . . . 84

6.2. The Manifold $X$.

6.3. Orientifold Limits of $X$.

6.3.1. Orientifold A . . . . . . . . . . . . . . . . . . . . . . . . . . 87

6.3.2. Orientifold B . . . . . . . . . . . . . . . . . . . . . . . . 88

6.3.3. Orientifold C . . . . . . . . . . . . . . . . . . . . . . . . 88

6.4. Generalization to Other Models _. . . . . . . . . . . . . . . . . . . 89

6.4.1. Other Classes of $G_{2}$ Manifolds _. . . . . . . . . . . . . . . . 90

6.4.2. "All" Orientifolds of type IIA on CY have a $G_{2}$ Limit . . . . . . 91

6.5. The Case of the Disappearing Orientifold . . . . . . . . . . . . . 93

6.6. Discussion . . . . . . . . . . . . . . . . . . . . . . . . . . 95

7. D(NA)-Branes . . . . . . . . . . . . . . . . . . . . . . . . . 98

7.1. Introduction . . . . . . . . . . . . . . . . . . . . . . . . . . 98

7.2. The quantum Hall soliton and its DNA dual . . . . . . . . . . . . 99

7.3. Probe analysis and low-energy dynamics . . . . . . . . . . . . 105

7.4. Low-energy theory on the strings . . . . . . . . . . . . . . . . 108

7.4.1. The full potential . . . . . . . . . . . . . . . . . . . . 109

7.5. Conclusions . . . . . . . . . . . . . . . . . . . . . . . . . 111

7.6. What we have to say about biophysics . . . . . . . . . . . . . 112

8. Geometric Constructions of Non-geometric String Theories . . 113

8.1. The Undiscover'd Country: weakly-coupled supersymmetric string vacua without geometry . . . . . . . . . . . . . . . . . . . . . . 114

8.1.1. The setup . . . . . . . . . . . . . . . . . . . . . . . . 115

8.2. Stringy cosmic fivebranes . . . . . . . . . . . . . . . . . . . 116

8.2.1. Type IIA string theory on $T^{2} \quad \ldots \quad$. . . . . . . . . . . . . . . 117

8.2.2. Killing spinors . . . . . . . . . . . . . . . . . . . . . . 118

8.2.3. $T^{2}$ over $\mathcal{B}_{2}$. . . . . . . . . . . . . . . . . . . . . . . . 120

8.2.4. Stringy cosmic fivebranes . . . . . . . . . . . . . . . . . 122

8.2.5. Putting things on top of other things . . . . . . . . . . . . 124

8.3. Compact models in six dimensions . . . . . . . . . . . . . . . 125

8.3.1. The spectrum of the $12+12^{\prime}$ model . . . . . . . . . . . . . 127

8.3.2. Other descriptions of these models . . . . . . . . . . . . 132

8.4. $T^{2}$ over $\mathcal{B}_{4} \quad$. . . . . . . . . . . . . . . . . . . . . . . . . 133

8.4.1. Spectrum . . . . . . . . . . . . . . . . . 133

8.5. Discussion . . . . . . . . . . . . . . . . . . . . . . . . . . 134

Appendix 8.A. Conventions about coordinates and indices . . . . . . 134

Appendix 8.B. Conventions for type IIA SUGRA . . . . . . . . . . 135

8.B.1. Corrections to $[177]$. . . . . . . . . . . . . . . . . . . . . . 135

8.B.2. Dictionary . . . . . . . . . . . . . . . . . . . . . . . 135

Appendix 8.C. Geometry of $T^{2}$ fibrations . . . . . . . . . . . . . . 137

Appendix 8.D. Derivation of the BPS Equations . . . . . . . . . . . 138

8.D.1. The other half of the Killing spinor equations . . . . . . . . 140 
Appendix 8.E. Vector modes in the base . . . . . . . . . . . . . . 141

Part III . . . . . . . . . . . . . . . . . . . . 144

9. Linear Sigma Models for Open Strings . . . . . . . . . . . . 144

9.1. Introduction . . . . . . . . . . . . . . . . . . . . . . . . . 144

9.2. Physical interpretation and phase structure . . . . . . . . . . . 146

9.2.1. Boundary fields . . . . . . . . . . . . . . . . . . . . . . 148

9.2.2. Review of bulk phase structure . . . . . . . . . . . . . . . 149

9.2.3. The Calabi-Yau phase . . . . . . . . . . . . . . . . . . . 150

9.2.4. The Landau-Ginzburg phase . . . . . . . . . . . . . . . . 151

9.2.5. A few words about quantum corrections . . . . . . . . . . . 151

9.3. Ingredients and Details . . . . . . . . . . . . . . . . . . . 152

9.3.1. B-type supersymmetric bulk terms . . . . . . . . . . . . . 153

9.3.2. Boundary multiplets . . . . . . . . . . . . . . . . . . . 155

9.3.3. Boundary terms in the action . . . . . . . . . . . . . 156

9.3.4. Boundary conditions . . . . . . . . . . . . . . . . . . . 157

9.3.5. Bulk multiplets on the boundary . . . . . . . . . . . . . . 160

9.3.6. Gauging the special symmetry . . . . . . . . . . . . . . . 161

9.3.7. The Warner problem . . . . . . . . . . . . . . . . . . . 162

9.3.8. Symmetries of bulk and boundary fields . . . . . . . . . . . 163

9.4. Sheafy variables . . . . . . . . . . . . . . . . . . . . . . . 164

9.4.1. Unions and intersections . . . . . . . . . . . . . . . . . . 167

9.5. Applications and consequences . . . . . . . . . . . . . . . . . . . 169

9.5.1. Monodromy in closed-string moduli space . . . . . . . . . . 169

9.5.2. Degenerations and Singularities . . . . . . . . . . . . . . . 171

9.5.3. Marginal stability transitions: A local worldsheet model . . . . 178

9.6. Massless worldvolume fields . . . . . . . . . . . . . . . . . . 181

9.6.1. Generalities . . . . . . . . . . . . . . . . . . . . . . . 181

9.6.2. Example: A bundle on $K 3$. . . . . . . . . . . . . . . . . . . . . 183

9.7. Future Directions . . . . . . . . . . . . . . . . . . . . . . 187

Appendix 9.A. Transformation properties of bulk supermultiplets . . . 188

Appendix 9.B. Boundary superspace . . . . . . . . . . . . . . . . 190

Appendix 9.C. The tensor formula . . . . . . . . . . . . . . . . . . 192

10. Linear Sigma Model Toolshed . . . . . . . . . . . . . . . . 194

10.1. Introduction _. . . . . . . . . . . . . . . . . . . . . . . 194

10.2. Brief review of the construction for one step . . . . . . . . . . 196

10.2.1. About kinetic terms . . . . . . . . . . . . . . . . . . . 197

10.2.2. The model for a monad . . . . . . . . . . . . . . . . . . 198

10.3. Any resolution . . . . . . . . . . . . . . . . . . . . . . . . . 199

10.3.1. Large-radius analysis . . . . . . . . . . . . . . . 203

10.3.2. Examples . . . . . . . . . . . . . . . . . 204

10.4. Bundles which do not extend to the ambient space . . . . . . 206

10.5. Discussion . . . . . . . . . . . . . . . . . . . . . . . . . 208

10.5.1. Relation to heterotic models . . . . . . . . . . . . 208

10.5.2. Other phases and singularities . . . . . . . . . . . . 209 
10.5.3. Other classes of models . . . . . . . . . . . . . . . . . . 210

Appendix 10.A. Full supersymmetry transformations and lagrangian . . 211

Appendix 10.B. Formulation with shift symmetries . . . . . . . . . . 213

11. Matrix Theory on Projective Space . . . . . . . . . . . . . 215

11.1. Introduction . . . . . . . . . . . . . . . . . . . . . . . . 215

11.2. Branes on $\mathbb{C P}^{1}$ (not really) . . . . . . . . . . . . . . . . 219

11.2.1. Techniques . . . . . . . . . . . . . . . . . . . . . . . 219

11.2.2. Worldvolume theory . . . . . . . . . . . . . . . 220

11.3. Branes on $\mathbb{C P}^{2}$ (not really) . . . . . . . . . . . . . . . . . . 222

11.3.1. Moduli space . . . . . . . . . . . . . . . . . . 223

11.3.2. Matricization . . . . . . . . . . . . . . . . . . . . 224

11.3.3. Branes on a compact CY! . . . . . . . . . . . . . . . . . 226

11.4. Branes on other toric surfaces . . . . . . . . . . . . . . . 226

11.4.1. Branes on $\mathbb{P}^{1} \times \mathbb{P}^{1}$. . . . . . . . . . . . . . . . . . . 226

11.4.2. Branes on $\mathbb{F}_{n}$. . . . . . . . . . . . . . . . . . . . . . . 227

11.4.3. Gauged shift symmetry . . . . . . . . . . . . . . . . . . 228

11.5. Reappearance of brane-antibrane constituents . . . . . . . . . . 229

11.6. Regrading . . . . . . . . . . . . . . . . . . . . . . . . . 230

11.7. Seiberg duality and its generalizations . . . . . . . . . . . . . 230

11.7.1. Tachyon condensation and the open string worldsheet . . . . 231

11.8. Conclusions . . . . . . . . . . . . . . . . . . . . . . . . 231 


\section{Chapter 1: Friendly Introduction}

String theory is a framework which reconciles the two main advances of twentieth century physics - quantum mechanics and Einstein's theory of gravity. It is a beautiful theory which has no ambiguity in its formulations, and which passes many non-trivial consistency tests. It has natural solutions which look very much like the world we observe; in addition to gravity, these solutions produce gauge theories with chiral matter, such as the Standard Model of particle physics.

In spite of all these laurels, we find our basic understanding of string theory at a rather primitive level. For example, just a few years ago, a large set of degrees of freedom were discovered in the theory whose significance had not been appreciated. These are extended objects, called D-branes, which are sources for the many tensor fields present in string theory (in the same way that an electron is a source for the electromagnetic field). Simply put, they are dynamical pieces of space on which open strings can end. An important feature of D-branes is that their motions are described by gauge theories (like the Standard Model mentioned above) which live on their worldvolume.

Our current understanding of string theory starts in ten dimensions with an enormous number of unbroken supersymmetries. Supersymmetry pairs particles with complementary partners of opposite statistics but the same mass. This constrains the behavior of the theory, and the number of such symmetries present in ten dimensions forbids many interesting stringy effects. The solutions of string theory which look something like our world generally involve curling up six of these dimensions into some very small shape which solves the equations of motion of the theory, leaving macroscopic the four dimensions which we observe. Furthermore, the shape of this "internal" space (known as a Calabi-Yau (CY) manifold) breaks most of the supersymmetries, which means that the dynamics become less constrained and we can see more inherently stringy phenomena. Besides providing a natural and tractable mechanism for breaking some of the supersymmetries, these curved backgrounds exhibit intriguing and fruitful connections between string theory and modern mathematics.

As mentioned above, (in flat space and at weak coupling) the dynamics of Dbranes have a simple description in terms of the sectors of free open strings which end on them. However, when a D-brane is embedded in curved space - such as 
one finds in some solutions of string theory which could be relevant to nature - or in the presence of other strong background fields, these open strings are strongly interacting. My work has focused on the fascinating behavior of D-branes in such environments. As I will explain below, D-branes provide many realizations of quasirealistic string vacua which can be studied using techniques which were previously unavailable. Furthermore, the dynamics of these objects in curved backgrounds have many remarkable qualitative consequences.

The class of examples of quasi-realistic string solutions alluded to above involves D-branes wrapping subspaces of a Calabi-Yau manifold and filling the noncompact dimensions we observe. The fluctuations of the branes could plausibly mediate the interactions of nature. Vacua of the heterotic string which look quite a bit like the real world have been known for some time. However, having at our disposal more ways of obtaining potentially phenomenological solutions gives new perspective on old problems, such as the relative strengths of the gravitiational and particle-physics forces, and the doublet-triplet splitting problem of grand-unified theories. These new vacua are in many cases dual to the old solutions, in the sense that the description in terms of branes is good in one regime of parameters, while a description via the heterotic string is valid in another, continuously connected regime. The brane description has some advantages. The realization in terms of Dbranes clearly delineates bundle moduli from moduli of the CY. Further, there exist in these backgrounds classical mechanisms for breaking supersymmetry which give the same desired scalings of masses as one finds from nonperturbative mechanisms used for quasi-realistic vacua of the (strongly coupled) heterotic string.

In the first part of this thesis, we discuss couplings between closed-string and open-string moduli in these backgrounds. In chapter two, we study an example where changing the closed-string moduli results in a jump in the spectrum of supersymmetric D-branes. We provide a local field theory model for this behavior.

In chapters three and four, we attempt to understand and use the duality between IIA with D6-branes wrapping a special Lagrangian (sL) three-cycle of a CY, $M$, and IIB with D5-branes wrapping the mirror holomorphic curve in the mirror manifold, $W$. On the both sides, one obtains massless scalars living in the four-dimensional spacetime from infinitesimal deformations of the supersymmetric 
cycles. On the IIB side, these deformations can classically be obstructed at some higher order; this can be summarized by a superpotential for the corresponding fields in the effective $4 \mathrm{~d}$ action. The result from classical geometry is exact in the sigma model (but we work at tree level in the string coupling). On the IIA side, however, a non-renormalization theorem shows that no potential for the deformation fields can be generated at any order of sigma-model perturbation theory. The classical superpotential on the IIB side must be reproduced instead by disc instantons - maps from the Euclidean string worldsheet to the CY whose images are holomorphic curves with boundary on the sL submanifold. We have shown that such configurations have the right properties to contribute to the superpotential.

We study in detail a particular class of examples of special Lagrangian 3-cycles and their mirror holomorphic curves. On the type IIA side, these involve CY manifolds which have genus $g$ curves of $A_{N}$ singularities. When these are resolved, one obtains families of rational curves. There exist deformations of the complex structure of the CY which break up these families into $N(2 g-2)$ isolated curves. The superpotential which encapsulates this effect is generated on the mirror by nonperturbative worldsheet effects. We have identified a mechanism by which the disc instanton sum cancels on the mirror of the locus where the nontrivial moduli space of curves exists. We analyzed the topology of the sL three-cycles and observed that their homology can vary as a function of closed-string moduli.

Part III describes techniques which can be applied to the study of these backgrounds. With S. Hellerman, S. Kachru and A. Lawrence, we formulated a new description of open strings ending on D-branes in CY manifolds. This description, known as a linear sigma model, is a two-dimensional field theory with tractable interactions which at long distances has the properties of the non-linear theory describing the strings' dynamics. Such an approach was used by E. Witten to great effect in describing closed-string interactions on CY manifolds. As with the closed string models, this approach allows one to gain quantitative understanding of the behavior of strings in regimes where the geometric description breaks down, such as when the CY space is very small or singular. Using this technology, one can determine the effective theory on the worldvolume of the branes, and in particular diagnose whether the this theory has chiral matter representations of interesting gauge groups. The linear model is an ideal tool for mapping out the space of vacua 
of the open strings, and for following the behavior of D-branes as the background geometry is deformed. In summary, the linear sigma models we have developed provide a powerful new technique for studying D-brane physics.

In the strong-coupling limit, these theories have a description in terms of an 11-dimensional theory, known as M-theory, compactified on a (possibly singular) manifold of $G_{2}$ holonomy. This fact allows a complementary approach to understanding these backgrounds via geometry and the (partly known) behavior of Mtheory at singularities. With S. Kachru, we examined this correspondence for some of the few known examples of compact $G_{2}$ manifolds. Our progress is described in chapter 5 .

Remarkably, some of the techniques which we have developed for describing the string worldsheet via linear sigma models are useful for making an effective theory of the fluctuations of branes - the supersymmetric structure of the interactions is similar. This has allowed us (with S. Hellerman) to develop field theories with finitely many fields describing probes of compact spaces. These studies could lead to nonperturbative definitions of string theory in these backgrounds, along the lines of the matrix model for M-theory. Our progress in this direction is described in chapter 10 .

One of the main obstacles to making further contact between string theory and observation is the apparent overabundance of solutions of the theory. Because of this embarassment of riches, it is important for string theorists to attempt to understand more basic aspects of the theory, even in regimes which are not directly interesting for phenomenology. A class of solutions of string theory about which much has been learned in the past few years involves a maximally-symmetric negatively curved space - Anti-de Sitter space, or $A d S$ for short - times a maximally-symmetric positively curved space - a sphere. The curvature of the sphere is supported against collapse by some tensor flux. It turns out that an understanding of certain simple aspects of the physics of these backgrounds follows directly from the behavior of D-branes in curved space and background fields. The main advance in this area was the realization that information about string theory in such backgrounds is contained in field theories which live on the boundary of the $A d S$ space. Using this duality, it was noticed that certain sets of states, in particular those with angular momentum larger than a cutoff set by the size of the space, were simply absent 
from the theory. Translating to a statement about string theory in $A d S$ times a sphere, this "Stringy Exclusion Principle" says that in certain vibrational modes, a string moving on the sphere has a maximum angular momentum. We (with L. Susskind and N. Toumbas) proposed a simple explanation of this phenomenon, as we will describe in more detail in chapter four.

The effect we discovered is similar to the behavior of an electric dipole in a magnetic field. Using earlier work by R. Myers, we showed that when a string moves through the background flux on the $p$-sphere, it becomes polarized and in fact expands into a spherical $D(p-2)$-brane. The key fact for understanding the missing states is that the size of this "giant graviton" grows with its angular momentum. The Exclusion Principle is simply understood as the fact that there is a biggest $(p-2)$-sphere inside a $p$-sphere.

The giant graviton effect is one way we can see that these objects which we have been studying (strings and D-branes) should have a more fundamental description than the perturbative one - in fact, they are made from the same stuff.

Further, our explanation of the exclusion principle is a manifestation of a new paradigm which has emerged for quantum gravity. In traditional particle physics, high energies were linked with short-distance phenomena. A quantum theory containing black holes problematizes this correspondence because a black hole grows as it gains energy. In quantum gravity it has become clear that the highest-energy objects are actually macroscopically large.

Above, we mentioned that realistic string vacua were generally of the form of a compactification of the ten-dimensional theory on a small Calabi-Yau manifold. Such vacua have the generic problem that the volume of the manifold is undetermined. The fact that classically there is no energy cost for changing this value leads to many phenenological problems. In chapter seven, we discuss a class of potentially realistic models which do not have that feature, because they cannot be described as a ten-dimensional geometry. We construct these vacua as two-torus fibrations where the monodromy group includes stringy symmetries. These theories are nevertheless weakly-coupled. They have a lower-dimensional local description as compactifications with various kinds of branes wrapping parts of the space.

The work we have been doing on strings and branes in curved backgrounds has many applications in string theory and M-theory. For example, the linear sigma 
model allows a very intuitive approach to off-shell string theory, in the following sense. The equations of motion of string theory are the vanishing equations for the beta functions of the worldsheet field theory; a massive theory such as the linear sigma model represents a configuration on the slope of a string theory potential hill. Very little is known about this off-shell configuration space. It is by developing a better understanding of such inner workings of the theory itself that we will learn to tackle the big questions which string theory must eventually address, such as vacuum selection, supersymmetry breaking, and the value and origin of the cosmological constant. 


\section{Chapter 2: Supersymmetric Three-cycles and (Super)Symmetry Breaking}

We describe physical phenomena associated with a class of transitions that occur in the study of supersymmetric three-cycles in Calabi-Yau threefolds. The transitions in question occur at real codimension one in the complex structure moduli space of the Calabi-Yau manifold. In type IIB string theory, these transitions can be used to describe the evolution of a BPS state as one moves through a locus of marginal stability: at the transition point the BPS particle becomes degenerate with a supersymmetric two particle state, and after the transition the lowest energy state carrying the same charges is a non-supersymmetric two particle state. In the IIA theory, wrapping the cycles in question with D6-branes leads to a simple realization of the Fayet model: for some values of the CY modulus gauge symmetry is spontaneously broken, while for other values supersymmetry is spontaneously broken.

\section{Introduction}

In the study of string compactifications on manifolds of reduced holonomy, odd-dimensional supersymmetric cycles play an important part (see for instance $[2,3,4,5,6,7,8,9,10,11]$ and references therein). In type IIB string theory, a supersymmetric three-cycle can be wrapped by a D3-brane to yield a BPS state whose properties are amenable to exact study; in the IIA theory or in M theory, Euclidean membranes can wrap the three-cycle and contribute to "holomorphic" terms in the low energy effective action of the spacetime theory (that is, terms that are integrated over only a subset of the fermionic superspace coordinates).

Of particular interest, partially due to their role in mirror symmetry $[6,8]$, have been special Lagrangian submanifolds in Calabi-Yau threefolds. In an interesting

The material in Chapter 1 appeared in "Supersymmetric Three-cycles and (Super)symmetry Breaking" with Shamit Kachru [1] and is reprinted with permission of Phys. Rev. D. 
recent paper by Joyce [11], various transitions which these cycles undergo as one moves in the complex structure or Kähler moduli space of the underlying CY manifold were described. In this note, we study some of the physics associated with the simplest such transitions discussed in $\S 6$ and $\S 7$ of [11]. These transitions are reviewed in $\S 2$. The physical picture which one obtains by wrapping D3-branes on the relevant cycles in IIB string theory is described in $\S 3$, while the physics of wrapped D6-branes in type IIA string theory occupies $\S 4$. Our discussion is purely local (in both the moduli space and the Calabi-Yau manifold), as was the analysis performed in [11]; we close with some speculations about more global aspects in $\S 5$.

At all points in this chapter, we will be concerned with rigid special Lagrangian three cycles. Since the moduli space of a special Lagrangian three cycle $N$ (including Wilson lines of a wrapped D-brane) is a complex Kähler manifold of dimension $b_{1}(N)[12,7]$, this means we have to focus on so-called "rational homology three spheres" with $H_{1}(N, \mathbb{Z})$ at most a discrete group. We will further assume that $H_{1}(N, \mathbb{Z})$ is trivial.

\section{Splitting Supersymmetric Cycles}

\subsection{Definitions}

Let $M$ be a Calabi-Yau threefold equipped with a choice of complex structure and Kähler structure. Let $\omega$ be the Kähler form on $M$, and let $\Omega$ be the holomorphic three-form, normalized to satisfy

$$
\frac{\omega^{3}}{3 !}=\frac{i}{8} \Omega \wedge \bar{\Omega}
$$

This also allows us to define two real, closed three forms on $M, \operatorname{Re}(\Omega)$ and $\operatorname{Im}(\Omega)$.

Let $N$ be an oriented real three-dimensional submanifold of $M$. We call $N$ special Lagrangian with phase $e^{i \theta}$ iff

a) $\left.\omega\right|_{N}=0$

b) $\left.(\sin (\theta) \operatorname{Re}(\Omega)-\cos (\theta) \operatorname{Im}(\Omega))\right|_{N}=0$

(a) and (b) together imply that

$$
\int_{N}(\cos (\theta) \operatorname{Re}(\Omega)+\sin (\theta) \operatorname{Im}(\Omega))=\operatorname{vol}(N)
$$

where $\operatorname{vol}(N)$ is the volume of $N$. 
Physically, the relevance of $\theta$ for us will be the following. Let $N$ and $N^{\prime}$ be threecycles which are special Lagrangian with different phases $\theta$ and $\theta^{\prime}$. Compactifying, say, IIB string theory on $M$, we can obtain BPS states which preserve half of the $\mathcal{N}=2$ spacetime supersymmetry by wrapping three-branes on $N$ or $N^{\prime}$. In the notation of [2], the surviving supersymmetries in the presence of a D3-brane on $N$, for example, are generated by

$$
\epsilon_{\delta}=e^{i \delta} \epsilon_{+}+e^{-i \delta} \epsilon_{-}
$$

with $\delta=-\frac{\theta}{2}-\frac{\pi}{4}$. For generic $\theta \neq \theta^{\prime}$, however, $N$ and $N^{\prime}$ preserve different $\mathcal{N}=1$ supersymmetries and the state with both wrapped three-branes would break all of the supersymmetry.

\subsection{Transitions}

The following supersymmetric three-cycle transitions are conjectured by Joyce to occur in compact Calabi-Yau threefolds $M$.

Choose two homology classes $\chi^{ \pm} \in H_{3}(M, \mathbb{Z})$ which are linearly independent in $H_{3}(M, \mathbb{R})$. For any $\Phi \in H^{3}(M, \mathbb{C})$, define

$$
\Phi \cdot \chi^{ \pm}=\int_{\chi^{ \pm}} \Phi
$$

Thus $\Phi \cdot \chi^{ \pm}$are complex numbers. Following Joyce, define a subset $W\left(\chi^{+}, \chi^{-}\right)$in $H^{3}(M, \mathbb{C})$ by

$$
W\left(\chi^{+}, \chi^{-}\right)=\left\{\Phi \in H^{3}(M, \mathbb{C}):\left(\Phi \cdot \chi^{+}\right)\left(\bar{\Phi} \cdot \chi^{-}\right) \in(0, \infty)\right\}
$$

So $W\left(\chi^{+}, \chi^{-}\right)$is a real hypersurface in $H^{3}(M, \mathbb{C})$.

Fix some small, positive angle $\epsilon$. For $\Phi \in H^{3}(M, \mathbb{C})$ write

$$
\left(\Phi \cdot \chi^{+}\right)\left(\bar{\Phi} \cdot \chi^{-}\right)=R e^{i \theta}
$$

where $R \geq 0$ and $\theta \in(-\pi, \pi]$. Then we say $\Phi$ lies in $W\left(\chi^{+}, \chi^{-}\right)$if $R>0$ and $\theta=0$. We say that $\Phi$ lies on the positive side of $W\left(\chi^{+}, \chi^{-}\right)$if $R>0$ and $0<\theta<\epsilon$. We say that $\Phi$ lies on the negative side of $W\left(\chi^{+}, \chi^{-}\right)$if $R>0$ and $-\epsilon<\theta<0$. Then, Joyce argues that the following kinds of transitions should occur. We are given a Calabi-Yau $M$ with compact, nonsingular three cycles $N^{ \pm}$in homology classes 
$\left[N^{ \pm}\right]=\chi^{ \pm} . N^{ \pm}$are taken to be special Lagrangian with phases $\theta^{ \pm}$. We assume $N^{ \pm}$intersect at one point $p \in M$, with $N^{+} \cap N^{-}$a positive intersection. As we deform the complex structure of $M$, the holomorphic three form moves around in $H^{3}(M, \mathbb{C})$ and therefore the phases $\theta^{ \pm}$of $N^{ \pm}$change.

When $[\Omega]$ is on the positive side of $W\left(\chi^{+}, \chi^{-}\right)$there exists a special Lagrangian threefold $N$ which is diffeomorphic to the connected sum $N^{+} \# N^{-}$, with $[N]=$ $\left[N^{+}\right]+\left[N^{-}\right]$in $H_{3}(M, \mathbb{Z}) . \quad N$ can be taken to be special Lagrangian with phase $\theta=0$ (this fixes the phase of $\Omega$ for us). As we deform $[\Omega]$ through $W\left(\chi^{+}, \chi^{-}\right), N$ converges to the singular union $N^{+} \cup N^{-}$. When $[\Omega]$ is in $W\left(\chi^{+}, \chi^{-}\right)$, the phases $\theta^{ \pm}$align with $\theta=0$. On the negative side of $W\left(\chi^{+}, \chi^{-}\right), N$ ceases to exist as a special Lagrangian submanifold of $M$ (while $\theta^{ \pm}$again become distinct).

For completeness and to establish some notation we will find useful, we briefly mention some motivation for the existence of these transitions [11]. In Joyce's model of the transition, there exists a manifold, $D$, with boundary $S \subset N$, which is special Lagrangian with phase $i$. If we call its volume $A$, this means that $i A=\int_{D} \Omega$. $S$ defines a 2-chain in $N$; since we are assuming that $H_{1}(N, \mathbb{Z})$ is trivial, by Poincaré duality, $S$ must be trivial in homology. Because $S$ is real codimension one in $N$, it actually splits $N$ into two parts:

$$
N=C^{+} \cup C^{-}, \partial C^{+}=-S, \partial C^{-}=S .
$$

So $C^{ \pm} \pm D$ define 3 -chains and in fact it turns out that

$$
\left[C^{ \pm} \pm D\right]=\chi^{ \pm}=\left[N^{ \pm}\right]
$$

We see that we can determine the volume of $D$ just from knowledge of $\chi^{ \pm}$:

$$
A=\frac{1}{i} \int_{D} \Omega=\int_{D} \operatorname{Im}(\Omega)=\int_{\chi^{+}} \operatorname{Im}(\Omega)
$$

using $\left.\operatorname{Re}(\Omega)\right|_{D}=0$ and $\left.\operatorname{Im}(\Omega)\right|_{N}=0$. But when $[\Omega]$ goes through $W\left(\chi^{+}, \chi^{-}\right)$, we see from (2.6) and from the definition of $W\left(\chi^{+}, \chi^{-}\right)$that $A$ becomes negative; at least in the local model in $\mathbb{C}^{3}$, this means that $N$ does not exist. 


\section{Formerly BPS States in IIB String Theory}

Now, consider Type IIB string theory compactified on $M$. When the complex structure is such that $[\Omega]$ is on the positive side of $W\left(\chi^{+}, \chi^{-}\right)$, one can obtain a BPS hypermultiplet by wrapping a D3-brane on $N$. One can also obtain BPS hypermultiplets by wrapping D3-branes on $N^{+}$or $N^{-}$.

Because

$$
[N]=\left[N^{+}\right]+\left[N^{-}\right]
$$

one can make a state carrying the same charges as the BPS brane wrapping $N$ by considering the two particle state with D3-branes wrapping both $N^{+}$and $N^{-}$. How does the energy of the two states compare?

Recall that the disc $D$ with boundary on $N$ splits $N$ into two components, $C^{ \pm}$. Define

$$
B^{ \pm}=\int_{C^{ \pm}} \Omega
$$

Then if we let $V$ denote the volume of $N$ and $V^{ \pm}$denote the volumes of $N^{ \pm}$, we recall:

$$
\begin{gathered}
V=B^{+}+B^{-} \\
V^{ \pm} e^{i \theta^{ \pm}}=B^{ \pm} \pm i A
\end{gathered}
$$

where $A$ is the volume of $D$. Since on this side of the transition $A$ is positive, $\theta^{+}$ is small and positive while $\theta^{-}$is small and negative. In fact, reality of the volumes $V^{ \pm}$lets us solve for $\theta^{ \pm}$in terms of $B^{ \pm}$yielding

$$
\theta^{ \pm}= \pm \frac{A}{B^{ \pm}}
$$

The energy of the single particle state obtained by wrapping a D3-brane on $N$ is $T_{D 3} \times V$ where $T_{D 3}$ is the D3 brane tension. The energy of the (nonsupersymmetric) state obtained by wrapping D3-branes on both $N^{ \pm}$can be approximated by $T_{D 3} \times$ $\left(V^{+}+V^{-}\right)$. Expanding (3.3) for small $\theta^{ \pm}$, we find:

$$
V^{+}+V^{-}=V+A\left(\theta^{+}-\theta^{-}\right)=V+A^{2}\left(\frac{1}{B^{+}}+\frac{1}{B^{-}}\right)
$$

So since $A>0$ and $\pm \theta^{ \pm}>0$ on this side of the transition, we see that the single wrapped brane on $N$ is energetically preferred. 
Therefore, when the complex structure is on the positive side of $W\left(\chi^{+}, \chi^{-}\right)$, the BPS state indeed has lower energy than the nonsupersymmetric two particle state carrying the same charges, by roughly $T_{D 3} \times A\left(\theta^{+}-\theta^{-}\right)$.

Now as one moves in the complex structure moduli space of $M$ through a point where $[\Omega]$ lies in $W\left(\chi^{+}, \chi^{-}\right), A$ and $\theta^{ \pm}$vanish. Therefore, (3.5) shows that that mass of the two particle state becomes equal to that of the single particle state: we are passing through a locus of marginal stability. On this locus, the two particle state consisting of branes wrapping $N^{ \pm}$is supersymmetric, since $N^{ \pm}$are special Lagrangian with the same phase.

Finally, move through to the region where $[\Omega]$ lies on the negative side of $W\left(\chi^{+}, \chi^{-}\right)$. Here, $\pm \theta^{ \pm}<0$. Since $N$ ceases to exist as a supersymmetric cycle, the two particle state with D3-branes wrapping $N^{ \pm}$is the lowest energy state carrying its charges. ${ }^{1}$ Note that the two particle state is nonsupersymmetric, since $N^{ \pm}$are special Lagrangian with different phases. Here, we are making the conservative assumption that there is no stable, nonsupersymmetric bound state of these two particles - such a bound state would be reflected in the existence of a (nonsupersymmetric) cycle in the homology class $\left[N^{+}\right]+\left[N^{-}\right]$with lower volume than $V^{+}+V^{-}$. This is tantamount to assuming that the force between the two particles is repulsive for slightly negative $A$. This is reasonable since for $A$ positive there is an attractive force and a (supersymmetric) bound state, and as $A$ decreases to zero the magnitude of the force and the binding energy decrease until they vanish when $A=0$.

This phenomenon is an interesting variant on the examples of [13]. There, a stable nonsupersymmetric state passes through a locus of marginal stability and becomes unstable to decay to a pair of BPS particles (which together break all of the supersymmetries). In the present example, a BPS particle becomes, as we move in complex structure moduli space, unstable to decay to a pair of BPS particles. Moving slightly further in moduli space, we see that the two BPS particles together break all of the supersymmetries.

1 In a global model, even if there do exist other supersymmetric cycles in the same class, there will be some region in moduli space close to the transition where the energy cost for moving to them in the Calabi-Yau will be larger than the energy gained. 


\section{D6-Branes and the Fayet Model}

Now, consider type IIA string theory on the Calabi-Yau $M$ in which the phenomena of $\S 2$ are taking place. Instead of studying particles in the resulting $\mathcal{N}=2$ supersymmetric theory, we wrap the three-cycle $N$ with a space-filling D6-brane (i.e., $3+1$ of the dimensions of the D6-brane fill the non-compact space). This yields an $\mathcal{N}=1$ supersymmetric theory in the non-compact dimensions. For simplicity (since all our considerations are local), we can assume $M$ is non-compact so we do not have to worry about cancelling the D6 Ramond-Ramond charge. Alternatively, we could imagine the model discussed below arising as part of a larger system of branes and/or orientifolds on $M$.

First, let's discuss the physics when $[\Omega]$ is on the positive side of $W\left(\chi^{+}, \chi^{-}\right)$. Since $b_{1}(N)=0, N$ has no moduli in $M$. Therefore, there are no moduli in the effective $3+1$ dimensional field theory on the wrapped D6-brane. The $U(1)$ gauge field on the brane survives reduction on $N$, so the $3+1$ dimensional low energy effective theory has a $U(1)$ gauge symmetry. Finally, because $N$ is a supersymmetric cycle with $H_{1}(N, \mathbb{Z})$ trivial, there is a unique supersymmetric ground state in the gauge theory (as opposed to a discrete set of ground states parametrized by Wilson lines around $N)$.

What about the physics when $[\Omega]$ is on the negative side of $W\left(\chi^{+}, \chi^{-}\right)$? The D6 which was wrapping $N$ has now split into two D6-branes, wrapping $N^{+}$and $N^{-}$. The $U(1)$ gauge field on each survives, yielding a $U(1)^{2}$ gauge theory. Because $N^{+}$and $N^{-}$are supersymmetric cycles with different phases, the theory has no supersymmetric ground state. We do expect a stable nonsupersymmetric ground state, as long as $[\Omega]$ is close enough to $W\left(\chi^{+}, \chi^{-}\right)$.

What is the physics associated with the phase transition when $[\Omega]$ lies in $W\left(\chi^{+}, \chi^{-}\right)$? At this point, the two D6-branes wrapping $N^{+}$and $N^{-}$preserve the same supersymmetry, and intersect at a point in $M$. Because the light states are localized at the intersection, the global geometry of the intersecting cycles doesn't matter and we can model the physics by a pair of flat special Lagrangian threeplanes intersecting at a point. This kind of system was discussed in [14], and using their results it is easy to see that the resulting light strings give rise to precisely one chiral multiplet with charges $(+,-)$ under the $U(1)^{2}$ gauge group of the two wrapped $\mathrm{D}$ branes. Therefore, one linear combination of the $U(1)$ s (the normal 
"center of mass" $U(1))$ remains free of charged matter, while the other (the "relative" $U(1))$ gains a single charged chiral multiplet $\Phi$. The relative $U(1)$ is therefore anomalous; [14] demonstrates that the anomaly is cancelled by inflow from the bulk.

Ignoring the center of mass $U(1)$ (which we identify with the surviving $U(1)$ on the positive side of $W$ ), the physics of this model is precisely reproduced by the Fayet model, the simplest model of spontaneous (super)symmetry breaking [15]. This is a $U(1)$ gauge theory with a single charged chiral multiplet $\Phi$ (containing a complex scalar $\phi$ ). There is no superpotential, but including a Fayet-Iliopoulos term $r D$ in the spacetime Lagrangian, the potential energy is

$$
V(\phi)=\frac{1}{g^{2}}\left(|\phi|^{2}-r\right)^{2}
$$

where $g$ is the gauge coupling.

The phase structure of the model is quite simple: For $r>0$, there is a unique supersymmetric minimum, and the $U(1)$ gauge symmetry is Higgsed. For $r<0$, there is a unique nonsupersymmetric minimum at $\phi=0$, so the $U(1)$ symmetry is unbroken. Precisely when $r=0$, there is a $U(1)$ gauge theory with a massless charged chiral field and a supersymmetric ground state.

Thus, we are led to identify the regions of positive, vanishing and negative $r$ with the positive side of $W\left(\chi^{+}, \chi^{-}\right)$, the locus where $[\Omega]$ is in $W$, and the negative side of $W$. The single real modulus which varies in the transition experienced by the supersymmetric three-cycle $N$ can be identified with the Fayet-Iliopoulos parameter $r$. This identification is consistent with the conjecture in [10] that in worldvolume gauge theories of A-type D-branes on Calabi-Yau spaces, complex structure moduli only enter as D-terms. ${ }^{2}$

\section{Discussion}

Exploration of the phenomena involving supersymmetric cycles in a Calabi-Yau manifold $M$ under variation of the moduli of $M$ has just started. It should be clear that as such phenomena are understood, they will have interesting implications

2 Note that the D6 branes in question here are considered A-type branes in the conventions of [10] since the three non-compact spatial dimensions are ignored. 
for the physics of D-branes on Calabi-Yau spaces (for a nice discussion of various aspects of this, see [10]).

One of the most enticing possibilities is that as more such phenomena are uncovered, we will find new ways to "geometrize" the study of supersymmetry breaking models in string theory. This would provide a complementary approach to attempts to write down interesting nonsupersymmetric string models informed by AdS/CFT considerations [16] or insights about tachyon condensation and nonsupersymmetric branes [17].

As a small step in this direction, it would be nice to find ways of going over small potential hills between different supersymmetric vacua of string theory. The transitions studied here, when put in the more global context of a manifold $M$ with (possibly) several supersymmetric cycles in each homology class, might provide a way of doing this. For instance in $\S 4$, as one moves $[\Omega]$ into the negative side of $W\left(\chi^{+}, \chi^{-}\right)$, it is clear that one is increasing the scale of supersymmetry breaking (at least in the region close to the transition). Suppose that after one moves through the negative side of $W$ in complex structure moduli space, eventually $N^{+}$and $N^{-}$ approach each other and intersect again and the phenomenon of $\S 2$ occurs in reverse, with a new supersymmetric cycle $N^{\prime}$ in the same homology class as $\left[N^{+}\right]+\left[N^{-}\right]$ popping into existence. In such a case, one would have a nonsupersymmetric ground state for some range of parameters on the negative side of $W$, and then eventually reach a supersymmetric ground state again (with the D6 brane wrapping $N^{\prime}$ ).

Similarly, on the negative side of $W$ there could exist "elsewhere" in $M$ a supersymmetric cycle $\tilde{N}$ in the same class as $\left[N^{+}\right]+\left[N^{-}\right]$. Although the cost in energy to move from wrapping $N$ to wrapping $\tilde{N}$ is nonzero and hence on the negative side of $W$ the phenomena of $\S 3, \S 4$ occur, eventually it may become advantageous for the D6 branes to shift over to wrapping $\tilde{N}$. This would again be a situation where supersymmetry is broken, and then restored, as one dials the complex structure modulus of the Calabi-Yau space.

\section{Acknowledgements}

We are grateful to J. Harvey, G. Moore and E. Silverstein for discussions. The research of S.K. is supported by an A.P. Sloan Foundation Fellowship and a DOE OJI Award. The research of J.M. is supported by the Department of Defense NDSEG Fellowship program. 


\section{Chapter 3: Open String Instantons and Superpotentials}

We study the F-terms in $\mathcal{N}=1$ supersymmetric, $d=4$ gauge theories arising from $D(p+3)$-branes wrapping supersymmetric $p$-cycles in a Calabi-Yau threefold. If $p$ is even the spectrum and superpotential for a single brane are determined by purely classical $\left(\alpha^{\prime} \rightarrow 0\right)$ considerations. If $p=3$, superpotentials for massless modes are forbidden to all orders in $\alpha^{\prime}$ and may only be generated by open string instantons. For this latter case we find that such instanton effects are generically present. Mirror symmetry relates even and odd $p$ and thus perturbative and nonperturbative superpotentials; we provide a preliminary discussion of a class of examples of such mirror pairs.

\section{Introduction}

The study of D-branes in Calabi-Yau threefolds is of both formal and phenomenological interest. As philosophical tools, Calabi-Yau threefolds provide a natural arena for studying nonperturbative stringy geometry. D-branes are excellent probes of this geometry as they are sensitive to structure well below the string scale (at weak string coupling) [19,20]. Furthermore a deep understanding of mirror symmetry requires understanding its action on D-branes [21,22,23].

The phenomenological interest is served by studying space-filling D-branes in type I or type II string theories, in configurations preserving $\mathcal{N}=1 \mathrm{SUSY}$ in four dimensions. ${ }^{3}$ These configurations fall into two classes [24,25]: 6-branes in type

The material in Chapter 2 appeared in "Open string instantons and superpotentials" with Shamit Kachru, Sheldon Katz, and Albion Lawrence [18] and is reprinted with permission of Phys. Rev. D.

3 The standard caveat is in force here: in order to satisfy Gauss' law for the various RR charges, one should either consider branes wrapping cycles in non-compact CalabiYau spaces, or consider configurations containing both branes and orientifolds. For our purposes the former assumption will suffice, but many of our considerations could also be applied to lower dimensional, non space-filling branes wrapping the same cycles. 
IIA wrapping special Lagrangian cycles of the threefold, and odd- $p$-branes in type I or type IIB wrapping even-dimensional cycles. The latter configurations (up to orientifolds) can be written as coherent sheaves on the threefold $[21,22,26]$ (or its mirror) and so involve the same type of data as heterotic compactifications $[27,28]$. The D-brane limit allows one to study gauge field data when it is intrinsically stringy (much as Landau-Ginzburg compactifications allow one to study intrinsically stringy aspects of geometric data), via open string techniques.

In closed-string compactifications on Calabi-Yau threefolds, worldsheet instanton effects are the most well-understood source of truly stringy physics. They drastically modify the geometry at short distances: in addition they lead to interesting physical effects such as the generation of nontrivial superpotentials in heterotic compactifications $[29,30] .{ }^{4}$ In this work we will study the effects of open-string instantons on D-brane physics, in particular on the superpotential. For branes wrapping even-dimensional cycles, we will find that the superpotential can be determined from classical geometry; for branes wrapping special Lagrangian cycles it is generated entirely by nonperturbative worldsheet effects.

This has interesting implications for mirror symmetry in the type II compactifications. To begin with, if a given mirror pair of cycles has massless deformations with a nontrivial superpotential, then the classical moduli spaces will not match under the mirror map. A holomorphic 2-cycle with its infinitesimal holomorphic deformations obstructed at some nontrivial order (so that a 5-brane wrapped around it will have massless chiral fields with a superpotential) will have as its mirror a 3 -cycle which has flat directions to all orders in $\alpha^{\prime}$. On the other hand, if we start with a special Lagrangian cycle and find that worldsheet instantons destabilize or make nonsupersymmetric the D-branes wrapping them, the mirror will respectively either not exist or will be some classically nonsupersymmetric configuration. This is reminiscent of a common feature of dualities of $\mathcal{N}=1$ gauge theories, where superpotentials generated by nonperturbative dynamics are dual to tree-level superpotentials [34]. However, it is a relatively novel situation for $\mathcal{N}=1$ dualities

4 In fact, it has been proved in [31] that the most easily realized heterotic $(0,2)$ models, those realized as gauged linear sigma models [32,33], are not destabilized by worldsheet instantons. As we will see in the following, it should be easier to find examples of D-brane models which exhibit disc instanton generated superpotentials. 
of string vacua, where normally instanton effects map to instanton effects, as in heterotic/F-theory duality [35]. Here mirror symmetry should provide a powerful tool for summing open string instantons, as it does for closed string worldsheet instantons [36]. Note that the nonperturbative superpotentials we are discussing here are not explicable in terms of gauge dynamics involving the (perturbative) D-brane gauge theory; we would expect in some circumstances the instanton effects will be related to gauge instantons of a non-perturbative D-brane gauge theory that arises at singular points in the brane moduli space. This is analogous to the fact that in heterotic $(0,2)$ models, worldsheet instantons can sometimes be related to gauge instantons of nonperturbative gauge groups arising from singular compactifications [37].

In this chapter we will begin to investigate disc instanton effects by asking whether nonperturbative superpotentials are generically generated by worldsheet (disc) instantons. We will find a story similar to that of the heterotic string [30]: when the open string instantons are isolated, nonvanishing (locally) runaway potentials may be generated. ${ }^{5}$

The plan of this chapter is as follows: in $\S 2$ we will review the construction of $\mathcal{N}=1$ four-dimensional theories via space-filling D-branes wrapped on CalabiYau threefolds in type II string theory. After discussing the results determined by classical geometry, we will discuss some constraints from string theory. First, at tree level the superpotentials are computable via topological open string theory, and the hypermultiplets of the background closed-string theory decouple [38]. Furthermore, we will find that in the case of D6-branes wrapping special Lagrangian three-cycles, superpotentials are forbidden not only classically but to all orders in $\alpha^{\prime}$, due to a Peccei-Quinn symmetry. Superpotentials in these cases can only be generated by topologically nontrivial disc instanton effects. In $\S 3$ we will discuss the generic superpotential terms that are allowed in the presence of an isolated holomorphic disc. Finally, in $\S 4$ we discuss promising directions for future work. Further results in explicit examples will appear in the next chapter [39].

There is a close relation between the ideas discussed in this chapter and earlier work of Witten [40] and Vafa [41]. As this work was being completed, we were also informed of the related work [42] by mathematicians.

5 However, in contrast to the heterotic string story, the classical moduli spaces of the brane configurations we will study are naturally compact. Hence, the superpotentials we find will have minima which are not "at infinity" in field space. 


\section{Classical geometry of D-branes on $\mathrm{CY}_{3}$}

We begin with D-brane configurations preserving four supercharges, to lowest order in $g_{s}$ and $\alpha^{\prime}$, in type II string theory on $M \times \mathbb{R}^{4}$ where $M$ is a Calabi-Yau threefold. We will assume that the D-branes fill all of spacetime so that we realize an $\mathcal{N}=1, d=4$ gauge theory.

The internal configurations preserving four supersymmetries fall into two classes [24,25]: "A-type" branes wrapping special Lagrangian submanifolds of the threefold, and "B-type" branes which wrap holomorphic cycles of the Calabi-Yau. (The latter may have nontrivial holomorphic gauge bundles living on them as well, corresponding to bound states with lower-dimensional branes. We will for the most part ignore this possibility.) In the present discussion these exist in the type IIA and IIB theories respectively. We will discuss the associated gauge theories of each class in turn.

\subsection{A-type branes}

\section{Spectrum}

In the large-volume, large-complex-structure limit, supersymmetric A-type branes wrap special Lagrangian submanifolds. Let such a manifold $\Sigma$ be described by a map

$$
f: \Sigma \rightarrow M .
$$

Recall that special Lagrangian submanifolds $\Sigma$ are defined by the properties that:

$$
\begin{aligned}
\operatorname{dim}_{\mathbb{R}} \Sigma & =\frac{1}{2} \operatorname{dim}_{\mathbb{R}} M \\
f^{*} \omega & =0 \\
f^{*}\left(\operatorname{Im} e^{i \theta} \Omega\right) & =0
\end{aligned}
$$

where $\omega$ is the Kähler form of $M, \Omega$ is the standard holomorphic $(3,0)$ form, and $e^{i \theta}$ is some phase.

$N$ D6-branes wrapping a single supersymmetric cycle $\Sigma \subset M$ have a $U(N)$ vector multiplet arising from massless open string excitations polarized completely in $\mathbb{R}^{4}$. Massless open string excitations polarized in $M$ form adjoint $U(N)$ chiral multiplets. We will focus on the case $N=1$. To lowest order in $\alpha^{\prime}$, the counting of massless chiral fields has been worked out. $\Sigma$ lives in a family of deformations 
with dimension $b_{1}(\Sigma)$ [43] (c.f. also [44] for a clear discussion). More precisely, each basis vector in the tangent space to the space of deformations may be used to construct a nontrivial harmonic one-form on $\Sigma$, and vice-versa. Of course the space of such deformations (which has real dimension $b_{1}(\Sigma)$ ) cannot make up our set of chiral multiplets which are built from complex scalars: for example $b_{1}(\Sigma)$ need not be even. However, deformations of flat connections of the D6-brane gauge field on $\Sigma$ also map one-to-one onto the space of harmonic one-forms on $\Sigma$, roughly because there is a Wilson line of the $U(1)$ gauge field around each 1-cycle. Thus for each element of the space $\mathcal{H}^{1}(\Sigma)$ of harmonic one-forms on $\Sigma$ one has two real flat directions which may be described by a complex scalar [23]. In other words, we find $b_{1}(\Sigma)$ massless chiral multiplets, one for each non-trivial one-cycle or harmonic one-form on $\Sigma$.

Note that if we have branes wrapping several (mutually supersymmetric) 3cycles, then we may get additional matter from any intersection points, in bifundamentals of the $U(1)$ s of each cycle. A local example of this was discussed in [1]. In this work we will discuss branes wrapping single "primitive" 3-cycles: however, as explained in [1], interesting transitions to this more complicated case can occur as one varies background (closed string) hypermultiplets.

A natural choice of coordinates on the moduli space of the wrapped D6 brane is the following $[41,45]$. Let $\left\{\gamma_{j}\right\}$ be a basis for $\mathcal{H}_{1}(\Sigma)$. Choose minimal area discs $D_{j}$ subject to the condition that

$$
\partial D_{j}=\gamma_{j}
$$

and let

$$
w_{j}=\int_{D_{j}} \omega .
$$

In other words, if there is a holomorphic disc in the relative homology class of $D$, then $w_{j}$ will be the area. The $w_{j}$ provide $b_{1}(\Sigma)$ real coordinates. They are complexified by $b_{1}(\Sigma)$ Wilson lines

$$
a_{j}=\int_{\gamma_{j}} A
$$

where $A$ is the $U(1)$ gauge field on the wrapped brane. The coordinates in (2.3) and (2.4) are the real and imaginary parts of scalar components of the $b_{1}(\Sigma)$ chiral multiplets $\Phi_{j}$ on the brane:

$$
\Phi_{j}=w_{j}+i a_{j}+\cdots
$$


where $\cdots$ indicates higher components of the superfields.

\section{Superpotentials and worldsheet instantons}

As noted above, it follows from McLean's theorem [43] that at lowest order in $\alpha^{\prime}$ the brane wrapping $\Sigma$ has a moduli space of dimension $b_{1}(\Sigma)$. So far there may still be $\alpha^{\prime}$ corrections which lift these flat directions. To lift moduli, one would need to generate either D-terms or F-terms in the low energy action; we will focus on the superpotential, since the chiral multiplets $\Phi_{j}$ are neutral (at least at generic points in the classical moduli space of the cycle) and do not appear in FI D-terms. At leading order in $\alpha^{\prime}$, the superpotential $W(\Phi)$ identically vanishes. We now determine to what extent $\alpha^{\prime}$ corrections of any sort are possible.

In fact, it turns out that there are no corrections to the open-string superpotential to any finite order in $\alpha^{\prime}$ : all contributions must come from nonperturbative corrections, arising from topologically nontrivial configurations. The arguments are almost identical to similar arguments for the heterotic string $[29,30]$. We will give two.

The first argument is a string theory argument. The (0)-picture vertex operator for a flat connection $A$ at zero momentum on the D6-brane has the form:

$$
V=\int_{\partial D} A_{\mu}(X) \partial_{\alpha} X^{\mu} d \sigma^{\alpha}
$$

where $X$ are coordinates on the brane and $\sigma$ coordinates on the worldsheet $D$. Let $A$ be polarized completely internally, so that it corresponds to a choice of Wilson lines around the elements of $H_{1}(\Sigma)$. If $X(\partial D)$ is a topologically trivial cycle on $\Sigma$, then $A$ can be written as an exact form $d \Lambda$. We see that $V$ vanishes after an integration by parts. Thus topologically trivial disc amplitudes give no non-derivative couplings (such as superpotential terms) of the imaginary parts of chiral multiplets, to all orders in $\alpha^{\prime}$. Holomorphy thus requires the superpotential vanish to all finite orders in $\alpha^{\prime}$.

If the boundary maps to a topologically non-trivial cycle $\gamma_{j} \subset \Sigma$, this argument fails. Such discs are non-trivial elements of the relative homology class $H_{2}(M, \Sigma)$. These worldsheets will give terms weighted by the instanton action:

$$
e^{-\left(w_{j}+i a_{j}\right) / \alpha^{\prime}}
$$


where $w_{j}$ is the spacetime area of this disc as in (2.3), and $a_{j}$ is its partner Wilson line (2.4). The $\alpha^{\prime}$ dependence is decidedly non-perturbative. Note that to obtain the contribution (2.7) to the action, the map $X(\sigma)$ must be a holomorphic map from the disc to $M$ with the desired boundary, and with the normal derivative to $X(\sigma)$ at the boundary taking values in the pullback of the normal bundle to $\Sigma$; this is a disc instanton. It follows from standard arguments that only such holomorphic maps have the correct zero mode count to contribute to a superpotential term in the spacetime theory.

The second argument is a spacetime argument. The space of $U(1)$ Wilson lines on a circle is the dual circle. Thus, any function of the chiral fields appearing in the effective action must be invariant under discrete shifts of their imaginary parts. Holomorphy then requires that the superpotential be a power series in $\exp \left(-\left[w_{j}+i a_{j}\right] / \alpha^{\prime}\right)$, which again gives a nonperturbative dependence on $\alpha^{\prime}$.

\section{Examples of three-cycles}

The best-known example arises in the Strominger-Yau-Zaslow formulation of mirror symmetry [23]: the claim is that any geometric Calabi-Yau with a geometric mirror can be written as a fibration of special Lagrangian $T^{3} \mathrm{~s}$. Mirror symmetry is fiberwise T-duality on these $T^{3} \mathrm{~s}$. D3-branes wrapping these fibers are mapped to D0-branes on the mirror. The $T^{3}$ has $b_{1}=3$ so all this is in accord with expectations: the mirror D0-brane and thus the wrapped D3-brane should have a 3-dimensional complex moduli space (which is the mirror threefold). Many examples of special Lagrangian three-cycles can be found as fixed loci of real structures. Some examples, which are homeomorphic to $\mathbb{R}^{3}$, are contained in $[24,38]$. Note that these have a $\mathbb{Z}_{2}$ Wilson line degree of freedom as $\pi_{1}\left(\mathbb{R P}^{3}\right)=\mathbb{Z}_{2}$.

In addition, there has been some discussion of local and non-compact models. Ref. [45] contains some general discussion of noncompact supersymmetric threecycles. A simple example with an isolated disc instanton is the following. Take $z_{1,2,3}$ as coordinates on $\mathbb{C}^{3}$, and choose $\omega$ and $\Omega$ to be the obvious Kähler form and holomorphic three-form. Then the three-cycle $\Sigma$ defined by

$$
\begin{gathered}
\left|z_{1}\right|^{2}-t=\left|z_{2}\right|^{2}=\left|z_{3}\right|^{2} \\
\operatorname{Im}\left(z_{1} z_{2} z_{3}\right)=0, \quad \operatorname{Re}\left(z_{1} z_{2} z_{3}\right) \geq 0
\end{gathered}
$$


with $t$ positive is special Lagrangian, and diffeomorphic to $S^{1} \times \mathbb{R}^{2}$. A generator $\gamma$ of $H_{1}(\Sigma)$ is given by the concrete choice

$$
\gamma:\left\{\left(t^{1 / 2} e^{i \theta}, 0,0\right)\right\}
$$

where $\theta$ runs from 0 to $2 \pi$. A holomorphic disc with boundary $\gamma$ and area $\pi t$ is given by

$$
\left.D_{t}:\left\{\left(z_{1}, 0,0\right),\left|z_{1}\right|^{2} \leq t\right)\right\}
$$

\subsection{B-type branes}

In the large-volume, large-complex-structure limit, supersymmetric B-type branes wrap holomorphic cycles of $M$. One may also examine bound states which can be described as gauge bundles on the highest-dimensional branes (c.f. for example [38] for a discussion). For simplicity we will focus on branes wrapping primitive cycles, and in our examples we will discuss cases where $\mathcal{C}$ is a rational curve.

For $N$ (space-filling) D-branes wrapping a given cycle $\mathcal{C} \subset M$ one again has a $U(N)$ vector multiplet arising from massless open strings polarized along the spacetime directions. Massless strings polarized along $M$ give rise to adjoint chiral multiplets. Again we will focus on $N=1$. For B-type branes wrapping $\mathcal{C}$ the infinitesimal supersymmetric deformations of the cycle are holomorphic sections of the normal bundle $\mathcal{N}_{\mathcal{C}}$. The number of such first-order deformations is therefore the dimension of the space of holomorphic sections, $H^{0}\left(\mathcal{C}, \mathcal{N}_{\mathcal{C}}\right)$ (this is the cohomology group of the bundle $\mathcal{N}_{\mathcal{C}}$, not a relative cohomology group). These are the scalars in the massless chiral multiplets. There is no guarantee that these deformations do not have an obstruction at higher order. ${ }^{6}$ Such obstructions, if they exist, correspond to elements of the group $H^{1}\left(\mathcal{C}, \mathcal{N}_{\mathcal{C}}\right)$ [46]. More specifically, given an element of the cohomology group $H^{0}\left(\mathcal{C}, \mathcal{N}_{\mathcal{C}}\right)$ one may try to construct a finite deformation by beginning with an infinitesimal deformation and constructing a finite deformation as a power series. $H^{1}$ measures the space of possible obstructions at each order in this series. Note that it may happen that although $H^{1}$ is nonvanishing, there is still a solution for this power series and thus a family of cycles. In the end, an

${ }^{6}$ For e.g. $\mathcal{C}$ a curve of genus $g \geq 1$, there are also $2 g$ Wilson line degrees of freedom which parametrize the flat $U(1)$ bundles on $\mathcal{C}$. These pair up into $g$ chiral multiplets and provide exactly flat directions. Similar comments apply if $\mathcal{C}$ is a four-cycle with $b_{1}(\mathcal{C}) \neq 0$. 
obstruction should appear as a higher-order term in the superpotential for a brane wrapped around this cycle [38].

Furthermore, deforming the complex structure of $M$ can also cause obstructions to (previously existing) deformations of $\mathcal{C}$. The basic statement is as follows (c.f. $[47,48])$. One may use the restriction map to $\mathcal{C}$ and the short exact sequence:

$$
\left.0 \rightarrow T_{\mathcal{C}} \rightarrow T_{M}\right|_{\mathcal{C}} \rightarrow \mathcal{N}_{\mathcal{C}} \rightarrow 0
$$

to write a map

$$
r: H^{1}\left(M, T_{M}\right) \rightarrow H^{1}\left(\mathcal{C}, \mathcal{N}_{\mathcal{C}}\right)
$$

If we perturb the complex structure of $M$ to first order by some element $\rho \in$ $H^{1}\left(M, T_{M}\right)$, a deformation of $\mathcal{C}$ exists which preserves $\mathcal{C}$ as a holomorphic cycle if and only if $r(\rho)=0$. Note that couplings of (open-string) chiral multiplets to (background closed-string) complex structure parameters in the superpotential are allowed and generic [38].

In the end, even counting these chiral multiplets is a harder problem on its face than for A-type branes, as the number of moduli depends not only on the intrinsic topology of the cycle but on the details of its embedding in $M$. (This is already apparent for rational curves in the quintic - c.f. [49,50].) Nonetheless, one may find a lot of specific examples for which computations are possible, especially for rational curves.

Some additional constraints exist as for A-type branes. First, the computation of the disc contribution to the superpotential can be reduced to a B-twisted open topological field theory calculation [38]. Again the Kähler parameters almost completely decouple from the superpotential: indeed, the computations of the dimension of $H^{0}\left(\mathcal{C}, \mathcal{N}_{\mathcal{C}}\right)$ and of the obstruction depend completely on the complex structure. But in addition, all contributions to B-model computations come entirely from constant maps into the target space $[51,40]$. There are no worldsheet instanton corrections and tree level sigma model calculations will suffice: the superpotential can be deduced from classical geometry. 
Examples of holomorphic curves and superpotentials

Many useful examples of holomorphic cycles exist in the literature. Several can be found or are referenced in [38]. We are particularly interested in two-cycles with nontrivial obstructed deformations.

The canonical example is simply a small resolution of the singular hypersurface in $\mathbb{C}^{4}$ :

$$
x y=z^{2}-t^{2 n}
$$

(Such a small resolution is consistent with the Calabi-Yau condition as the space is noncompact.) If $n=1$, then $H^{0}\left(\mathcal{C}, \mathcal{N}_{\mathcal{C}}\right)=0$, and the curve is rigid. If $n>1$, then $H^{0}\left(\mathcal{C}, \mathcal{N}_{\mathcal{C}}\right)$ is one-dimensional - the normal bundle to this curve is $\mathcal{O}(0) \oplus \mathcal{O}(-2)$ - but there is an obstruction at $n$th order to deforming this curve [52]. This phenomenon can be described by a superpotential $W(\Phi)=\Phi^{n+1}[38]$.

It is easy to use $W(\Phi)$ to see the effect of a general deformation of complex structure on $C$. We perturb $W(\Phi)$ to

$$
W_{t}(\Phi)=\Phi^{n+1}+t P(\Phi)+O\left(t^{2}\right)
$$

where $P(\Phi)$ is an arbitrary polynomial in $\Phi$ subject only to the genericity condition $P^{\prime}(0) \neq 0$. Solving $W_{t}^{\prime}(\phi)=0$, we get $n$ solutions for the vev

$$
\phi_{k}(t)=e^{2 \pi i k / n}\left(-\frac{P^{\prime}(0)}{n+1}\right)^{1 / n} t^{1 / n}+\ldots
$$

where the dots denote higher order terms in $t$. The geometric description of this perturbation of curves with normal bundle $\mathcal{O}(0) \oplus \mathcal{O}(-2)$ to $n$ rigid rational curves was well known [53]. The geometric perturbation of contractible curves with normal bundle $\mathcal{O}(1) \oplus \mathcal{O}(-3)$ to rigid curves has recently been worked out in [54] and can be rephrased in terms of the perturbation of a superpotential if desired. The geometric description in the case of a general $\mathcal{O}(1) \oplus \mathcal{O}(-3)$ curve is not yet worked out, but the introduction of a superpotential can be expected to clarify the geometry.

\section{Digression on holomorphic Chern-Simons theory}

Another way to arrive at the superpotential in examples like (2.14), (2.15) is by studying a holomorphic analogue of the Chern-Simons action, discussed in 
$[40,41] \cdot{ }^{7}$ In the following, we will suppress constants which enter harmlessly in our formulas. We think of the Calabi-Yau $M$ as being obtained from the total space of the normal bundle $\mathcal{O}(0) \oplus \mathcal{O}(-2)$ by a modification of the complex structure. We choose holomorphic coordinates $\left(z, z_{0}, z_{1}\right)$ on the normal bundle, with $z$ being a coordinate on $\mathcal{C}, z_{0}$ being in $\mathcal{O}(0)$, and $z_{1}$ in $\mathcal{O}(-2)$. The curve $\mathcal{C}$ is identified with the zero section $z_{0}=z_{1}=0$. The modification of complex structure is realized as usual by perturbing $\bar{\partial}$ by a tensor $A_{\bar{j}}^{i}$, i.e. $\bar{\partial}_{\bar{j}} \mapsto \bar{\partial}_{\bar{j}}+A_{\bar{j}}^{i} \partial_{i}$, where $A_{\bar{j}}^{i}$ is a $T M$ valued $(0,1)$ form on $M$. We assume that the curve $\mathcal{C}$ remains holomorphic, and want to understand which deformations $z_{i}=\phi_{i}(z)(i=0,1)$ remain holomorphic. The space of $C^{\infty}$ deformations of $\mathcal{C}$ is identified with the space $\left(\phi_{0}, \phi_{1}\right)$ of $C^{\infty}$ sections of the normal bundle $\mathcal{N}_{\mathcal{C}}$. The relevant holomorphic Chern-Simons action is

$$
\int_{\mathcal{C}}\left(\phi_{0}\left(\bar{\partial}+A_{\bar{z}}^{i} \partial_{i}\right) \phi_{1}-\phi_{1}\left(\bar{\partial}+A_{\bar{z}}^{i} \partial_{i}\right) \phi_{0}\right) \text {. }
$$

Note that in (2.16) we only use the index $\bar{j}=\bar{z}$ in $A$. (2.16) expands as

$$
\int_{\mathcal{C}} \phi_{0} \bar{\partial} \phi_{1}+\phi_{0} A_{\bar{z}}^{z} \partial \phi_{1}+\phi_{0} A_{\bar{z}}^{1}-\left(\phi_{1} \bar{\partial} \phi_{0}+\phi_{1} A_{\bar{z}}^{z} \partial \phi_{0}+\phi_{1} A_{\bar{z}}^{0}\right)
$$

We make sense of this by respectively identifying $\phi_{0}, \phi_{1}$ with functions and $(1,0)$ forms on $\mathcal{C}$ (as would be expected in the twisted brane worldvolume theory [55]), while respectively identifying $A_{\bar{z}}^{0}, A_{\bar{z}}^{1}$ with $(0,1)$ and $(1,1)$ forms after pulling back to $\mathcal{C}$. Thus all the terms in $(2.17)$ are $(1,1)$ forms on $\mathcal{C}$ and can be integrated.

The variations of $(2.16)$ or $(2.17)$ with respect to $\phi_{0}$ and $\phi_{1}$ give the conditions that the corresponding curve in $X$ is holomorphic. In fact, the action of the topological theory on $\mathcal{C}$ actually becomes the superpotential in the four-dimensional $\mathcal{N}=1$ theory arising from wrapping a D5 brane on $\mathcal{C}$. This is because the holomorphic Chern-Simons theory is the string field theory for the open string topological B-model [40], and therefore its action is the generating function of the topological correlation functions which give rise to superpotential terms in the physical theory.

To illustrate this fact, we now show that we can choose our tensor $A$ so that (2.17) becomes $W(\Phi)=\Phi^{n+1}$. Since the obstructions to deforming $\mathcal{C}$ lie in $H^{1}\left(\mathcal{N}_{\mathcal{C}}\right)=H^{1}(\mathcal{O}(-2))$, we choose our $A$ to have $A_{\bar{z}}^{1}=z_{0}^{n} d z \wedge d \bar{z}$ while the

7 We thank C. Vafa for pointing this out to us, and D. Diaconescu for related discussions. 
other $A_{\bar{z}}^{i}$ vanish (we can always choose such a gauge). Then the constant section $\left(\phi_{0}, \phi_{1}\right)=(t, 0)$ is holomorphic provided we put $t^{n}=0$. So this $A$ produces the required geometry.

The variation of (2.17) with respect to $\phi_{1}$ shows that $\phi_{0}$ is holomorphic. The variation of (2.17) with respect to $\phi_{0}$ shows that $\bar{\partial} \phi_{1}$ is a multiple of $\phi_{0}^{n}$. Substituting these back into (2.17) (and performing the integral over the curve $\mathcal{C}$, which just produces a volume factor) gives a multiple of $\phi_{0}^{n+1}$, as claimed. This proves that for any $\mathcal{O}(0) \oplus \mathcal{O}(-2)$ curve, the superpotential will be a polynomial of degree $k$ for some $k$ (or will vanish identically) $-k$ is the only invariant of the complex structure in some neighborhood of the curve.

\section{Another Example}

Another example which we will use was detailed in Ref. [56] (see also sec. 9 of [48].) Here one has at a specific point in the complex structure moduli space an $A_{1}$ singularity fibered over a genus-g curve $\mathcal{S}$. At this point the collapsing cycles obviously form a family which is precisely $\mathcal{S}$. Deformations of the complex structure of $M$ destroy this family, generically leaving $2 g-2$ isolated curves. One may find $2 g$ three-cycles by sweeping the collapsing curves over the one-cycles of $\mathcal{S}$, mapping $H_{1}(\mathcal{S})$ to $H_{3}(M) .{ }^{8}$ This can be lifted to a map from $H^{(1,0)}(\mathcal{S})$ into $H^{(2,1)}(M) \simeq$ $H^{1}\left(M, T_{M}\right)[56,48]$. This gives $g$ independent first-order deformations of complex structure. $^{9}$ We can use the map $r$ (2.13) to project the relevant deformation onto $H^{1}(\mathcal{N})$ for each fiber of this collapsing surface. Now the spaces $H^{1}(\mathcal{N})$ are the fibers of a bundle over $\mathcal{S}$, and this bundle is identified with the canonical bundle of $\mathcal{S}$. So (2.13) gets included in the sequence of maps

$$
H^{(1,0)}(\mathcal{S}) \rightarrow H^{1}\left(M, T_{M}\right) \rightarrow H^{0}\left(\mathcal{S}, K_{\mathcal{S}}\right)
$$

8 This is closely related to the formula for the superpotential in [57]. Fixing a point $s_{0} \in \mathcal{S}$, then a path from $s_{0}$ to $s \in \mathcal{S}$ sweeps out a 3 -chain in $M$, which can be integrated over a holomorphic 3-form, defining a function of $s$. If we define the potential this way in our context, there is a multiplicative ambiguity from the choice of holomorphic 3 -form, reflected in the description in the main text by the choice of isomorphism $H^{(2,1)}(M) \simeq$ $H^{1}\left(M, T_{M}\right)$.

9 If $M$ arises from Batyrev's construction of Calabi-Yau toric hypersurfaces by blowing up the curve $\mathcal{S}$ of $A_{1}$ singularities, these deformations of the complex structure of $M$ are those which are not realizable by polynomial/toric deformations. 
This says two things. First of all, first order differentials on $\mathcal{S}$ lead to first order deformations of complex structure, realizing $g$ deformations of complex structure. Second, upon perturbing by such a complex structure deformation, the only curves which survive the deformation are those which are located at zeros of the associated section of $K_{\mathcal{S}}$. Thus generally we will find a set of isolated curves with only massive chiral multiplets. However, at codimension one in the complex structure moduli space, zeros of the section of $K_{\mathcal{S}}$ will coincide and the resulting curve will have higher multiplicity: their deformations will be massless but obstructed at some higher, non-trivial order.

A superpotential which reflects this geometry be constructed as follows. Let the complex structure deformation be induced as above from an element $\omega \in H^{(1,0)}(S)$. At $\omega=0$ the curve lives in a family which is precisely $\mathcal{S}$ so that a deformation of a curve at $z \in \mathcal{S}$ described by $\mathcal{N}$ can also be written as an element $\phi \in T_{z}^{(1,0)} \mathcal{S}$. One may then write the superpotential as:

$$
W(\Phi ; \omega)=\langle\omega, \Phi\rangle+\frac{1}{2 !}\langle\partial\langle\omega, \Phi\rangle, \Phi\rangle+\frac{1}{3 !}\langle\partial\langle\partial\langle\omega, \Phi\rangle, \Phi\rangle, \Phi\rangle+\cdots
$$

Here $\Phi$ is the superfield associated to $\phi, \partial$ is the the Dolbeault operator on $\mathcal{S}$ and $\langle$, is the usual inner product between forms and vectors. It is understood that one is to evaluate the inner product at the point $p \in \mathcal{S}$ around which one is expanding, and convergence follows from the convergence of the power series representation of $\omega$. For $\mathcal{S}$ of genus $g$, the expansion (2.19) can be truncated after $2 g-1$ terms without changing the location and structure of the critical points. The closed string complex structure moduli act as parameters in the superpotential, through the choice of $\omega$.

Let us explore the properties of (2.19) slightly more explicitly, to illustrate its features. Consider expanding (2.19) about some point on $\mathcal{S}$ where $\omega$ has an expansion in a local complex coordinate $z$

$$
\omega \sim z^{n} d z
$$

We can represent the scalar field, which we are thinking of as a tangent vector to $\mathcal{S}$, as $\phi \frac{\partial}{\partial z}$ with $\phi$ complex. Then, expanding (2.19) around $z=0$, we find

$$
W(\Phi) \sim \Phi^{n+1} .
$$


For $n=0$ (i.e. around generic points on $\mathcal{S}$ ) there is no supersymmetric vacuum, while for $n>0$ there are supersymmetric vacua. For $n=1$, the vacuum is massive; for $n>1$ there is a massless field, and the vacuum splits into $n-1$ massive vacua upon small perturbations of the complex structure of $M$ (just as in the situation of $(2.15))$. For $\mathcal{S}$ of genus $g, \omega$ will generically have $2 g-2$ isolated zeroes, giving rise to $2 g-2$ massive supersymmetric vacua at generic points in the space of background closed string parameters. At various codimensions in the closed string moduli space, as one further specializes the multiplicities of the zeroes of $\omega$, these $2 g-2$ vacua merge in various combinations to yield theories with massless fields obstructed by higher order potentials.

A simpler way to write (2.19) locally on $\mathcal{S}$ is to write $\omega=d f_{\omega}$ for a locally defined function on $\mathcal{S}$. Locally, such an $f$ can be thought of as a function of $\phi$. Then we simply have

$$
W(\Phi, \omega)=f_{\omega}(\Phi)
$$

While this formula is simpler in form than (2.19), it does not capture the global structure of the moduli space $\mathcal{S}$.

The above considerations are easily adapted to the more general situation considered in [56], where an $A_{N}$ singularity is fibered over $\mathcal{S}$. If we denote the collapsing curve as $C_{1} \cup \ldots \cup C_{N}$, then for each $C_{j}$ we get $g$ deformations of complex structure arising as in (2.18), yielding $g N$ complex moduli. But we also have connected subsets $C_{k} \cup C_{k+1} \cup \ldots \cup C_{k+r}$ to which the above analysis applies. But since the first map in (2.18) depends linearly on the individual $C_{j}$, including these connected subsets does not give rise to any new complex structure deformations. So we get $N(N+1) / 2$ superpotentials of the form (2.19) on $N(N+1) / 2$ copies of $\mathcal{S}$, each of which depends on the $g N$ complex moduli (only $g$ of which appear in any one superpotential). Each of these superpotentials controls the obstructions to deforming curves of the form $C_{k} \cup C_{k+1} \cup \ldots \cup C_{k+r}$, and $2 g-2$ curves of this type survive a generic deformation of complex structure.

\section{Disc instantons}

Type II string theory in the presence of a D-brane on a given special Lagrangian submanifold has the same net number of worldsheet (and spacetime) supersymmetries as a heterotic $(0,2)$ model; and as with heterotic $(0,2)$ models, the 
nonrenormalization theorem for the spacetime superpotential is spoiled by worldsheet instanton effects. In light of results for $(0,2)$ models [30], it is fair to ask whether the generic D6-brane configuration is nonperturbatively stable.

We expect direct calculations of instanton effects to be difficult. But instantons in supersymmetric theories generate fermion zero modes which provide selection rules for CFT correlators. Using the rule of thumb that allowed terms are generic, we will see that three-cycles with an isolated disc instanton are destabilized nonperturbatively. ${ }^{10}$ The argument is quite similar to that for heterotic $(0,2)$ models.

The easiest way to count the zero modes for an isolated holomorphic disc is to begin with the amplitude for the sphere and get the disc by orbifolding with respect to a real involution, which will cut the number of zero modes in half. For an isolated sphere, the superconformal symmetry together with an index theorem shows that there are four holomorphic zero modes and four antiholomorphic zero modes [30,58], so we expect four fermion zero modes on the disc.

Consider a single D6-brane wrapping a special Lagrangian three-cycle $\Sigma$. The complex modulus $\phi=w+i a$ is associated with a cycle $\gamma \in H_{1}(\Sigma)$, using the notation and definitions of $\S 2.1$. Here we assume the isolated instanton corresponds to a disc $D$ such that $\partial D=\gamma$ and $D$ has minimal area. The most obvious, lowest-order term consistent with our perturbative nonrenormalization theorem is the exponential

$$
W(\Phi)=e^{-\Phi / \alpha^{\prime}}
$$

where $\Phi$ is the superfield corresponding to $\phi$. This will clearly destabilize the wrapped D6-brane, at least locally.

We will search for the superpotential (3.1) by examining small fluctuations $\Phi_{j}$ away from the above classical configuration $\Phi_{0}=\phi$. Here $j$ is an index in $H_{1}(\Sigma)$. The lowest-order terms directly computable via a CFT correlator will be those arising from the cubic term

$$
\Phi_{i} \Phi_{j} \Phi_{k} e^{-\phi / \alpha^{\prime}}
$$

We will focus on the term

$$
S_{\text {cubic }}=C \int d^{4} x \phi_{i} \phi_{j} F_{k}
$$

10 Holomorphic discs ending on special Lagrangian cycles of Calabi-Yau threefolds are generically isolated [40]. 
where $F_{k}$ is the auxiliary field in $\Phi_{k}$. Note that in the reduction to four dimensions, the operators above are arrived at by contour integrals in $\Sigma$, so $C$ is proportional to a triple integral.

The vertex operators which enter in the calculation of (3.2) are easily presented in the covariant RNS formalism [59] (c.f. [38] for a general discussion of the CFT calculation of open-string superpotential terms). The (-1)-picture zero-momentum vertex operator for the scalar component $\phi_{j}$ is:

$$
V_{\phi}^{(-1), j}=\theta_{\mu}^{j}(X) \psi^{\mu} e^{-\tilde{\phi}}
$$

where $\tilde{\phi}$ is the bosonized superconformal ghost [59], $\theta_{\mu}^{j}$ is the harmonic one-form (associated to $\gamma_{j}$ ) on the 3 -cycle, and $\psi^{\mu}$ is a fermion with Dirichlet boundary conditions. The (0)-picture vertex operator for the auxiliary field is [60]:

$$
V_{F}^{(0), j}=\Omega_{\rho \mu \nu}(X) \theta_{\sigma}^{j} g^{\sigma \rho} \psi^{\mu} \psi^{\nu}
$$

Here $\Omega$ is the the $(3,0)$ form, with the coordinates (but not the indices) restricted to $\Sigma$. Equation (3.4) is obtained by applying the unit spectral flow operator $\Omega_{\mu \nu \rho} \psi^{\mu} \psi^{\nu} \psi^{\rho}$ as in $[60,28]$.

The three-point function

$$
\left\langle V_{\phi}^{(0), i} V_{\phi}^{(-1), j} V_{F}^{(-1), k}\right\rangle
$$

has the correct fermion and ghost number to be nonvanishing; in an instanton background, the four fermions in the vertex operators in (3.5) can soak up the relevant zero modes. Note that we are computing the integrand of the triple integral defining $C$ in Eq. (3.2). Since holomorphic maps will preserve the order of marked points on the boundary, the ordering of (3.5) will be fixed for a given set of positions in this integrand.

This superpotential term can equivalently be computed as a correlator in the topological A-model open string theory $[40,38]$. Here one is computing the contribution to (3.5) (or more familiarly, a Yukawa coupling related to (3.5) by supersymmetry) in a sector where the map of the worldsheet to spacetime is a disc whose boundary $\gamma \subset \Sigma$ is topologically nontrivial. The path integral localizes onto the space of holomorphic maps, and the contribution is

$$
\oint_{\gamma} \oint_{\gamma} \oint_{\gamma} d x_{1} d x_{2} d x_{3} A_{i}\left(x_{1}\right) A_{j}\left(x_{2}\right) A_{k}\left(x_{3}\right)
$$


(suppressed by the exponential of the area of the holomorphic disc), where the gauge fields $A_{i}$ can be identified with the 1 -forms $\theta^{i}$ in (3.3). Once again, for given positions in the integrand, the ordering of the vertex operators for $A_{i, j, k}$ must match the ordering of $x_{1,2,3}$ respectively.

The result is that the superpotential (3.1) is generic for an isolated instanton. With some interpretation added, this statement matches a calculation in ref. [40]. There it is shown that the string field theory for the topological open string A-model is equivalent to Chern-Simons theory on $\Sigma$ with instanton corrections to the action. This instanton correction can be interpreted as precisely the superpotential we have calculated, as it generates the topological correlator we have discussed. Note that in [40], the dependence on the area of the disc was added as a convergence factor, whereas in our discussion it is required by spacetime supersymmetry.

The topological string theory representation of the superpotential allows us to write the full worldsheet instanton contribution to the CFT correlator (3.5). First, note that while we have discussed $A_{i}$ as a harmonic form, we can modify it by adding a BRST-trivial piece to give it support only in an arbitrarily small neighborhood around a two-cycle $\beta_{i}$ which is Poincaré dual to $\gamma_{i}$. The result is as follows. Denote by $d_{\left\{m_{l}\right\}}^{\left\{n_{a}\right\}}(i, j, k)$ the number of holomorphic maps from a disc to $M$ where the image $D \subset M$ has the following properties: ${ }^{11}$

i) $[\partial D]=\sum_{l} m_{l} \gamma_{l}$.

ii) The vertex operators $V^{i, j, k}$ are mapped in cyclic order to intersections of $\gamma=\partial D$ with $\beta_{i, j, k}$ respectively. ${ }^{12}$

iii) $\quad D-\sum_{l} m_{l} D_{l}$, which is a closed two cycle in $M$, is in the homology class $\sum_{a} n_{a} K_{a}$.

11 As with "numbers" of rational curves in mirror symmetry, the correct notion of $d$ when there are families of discs and/or including multiple covers would require much further discussion; we will be content here to be schematic. A proposal for the multiple cover contribution has recently been worked out by H. Ooguri and C. Vafa [61].

12 One has to be careful if two vertex operators correspond to the same cycle. The support of $A$ can be made arbitrarily small but finite. In this way nonzero contributions still generically come from the vertex operator insertions mapping to different points in $\gamma$. 
Then, the three-point function receives a contribution

$$
\begin{aligned}
\left\langle V_{F}^{i} V_{\phi}^{j} V_{\phi}^{k}\right\rangle \sim \sum_{m_{l}, n_{a} \geq 0} & \left(\int_{\partial D} \theta^{i}\right)\left(\int_{\partial D} \theta^{j}\right)\left(\int_{\partial D} \theta^{k}\right) \times \\
& \times d_{\left\{m_{l}\right\}}^{\left\{n_{a}\right\}}(i, j, k) \prod_{l=1}^{b^{1}(\Sigma)} e^{-m_{l}\left(w_{l}+i a_{l}\right) / \alpha^{\prime}} \prod_{a=1}^{h^{1,1}(M)} e^{-n_{a} t_{a}}
\end{aligned}
$$

from disc instantons, where $t_{a}$ denotes the integral of the Kähler form over $K_{a}$ (and for simplicity we are setting the closed string background $B$-field to zero). Although we have mostly used the language of the topological theory in deriving this result, it also holds for the three-point function in the physical theory.

The same kind of instanton sum also appears in [41], where the interpretation in terms of a superpotential for wrapped branes (and in particular the fact that these effects serve to obstruct the deformations of branes wrapped on special Lagrangian cycles) was not discussed. 


\section{Coupling to closed string background fields}

It is clear from the form of the three-point functions (3.7) that the superpotential depends on the closed string background Kähler moduli, which enter through the worldsheet instanton action $e^{-t_{a}}$. The dependence of the disc instanton generated superpotential on Kähler moduli, and the fact that it does not depend on the background complex structure moduli in the IIA theory, is consistent with the nonrenormalization result of [38].

We can directly probe the dependence of the superpotential on closed string moduli by computing the CFT correlator

$$
\left\langle V_{K}^{(-1,-1), a} V_{F}^{(0), j}\right\rangle
$$

where $V_{K}^{(-1,-1), a}$ is the vertex operator for a closed string Kähler deformation. Again, the vertex operators in (3.8) can absorb the fermion zero modes which are present in an instanton background. In fact, the "mirror" couplings of open strings to background complex moduli in the superpotential generically exist at tree level in the B-model [38] - this is clear from the examples of $\S 2.2$, where a small perturbation of complex structure can obstruct families of holomorphic curves. The couplings (3.8) must then similarly exist, but due to Peccei-Quinn symmetries they should arise at the non-perturbative level in both the closed and open string worldsheet instanton expansions.

$V_{K}^{a}$ represents an integral $(1,1)$ form $\omega_{a}$ which could be used to perturb the Kähler form of $M$. We can choose $\omega_{a}$ to have support only infinitesimally near the four-cycle $L^{a} \subset M$ Poincare dual to $K_{a}$. Then, (3.8) has the expansion

$$
\begin{aligned}
\left\langle V_{K}^{(-1,-1), a} V_{F}^{(0), j}\right\rangle \sim & \sum_{m_{l}, n_{b} \geq 0}\left(\int_{D} \omega_{a}\right)\left(\int_{\partial D} \theta^{j}\right) \times \\
& \times d_{\left\{m_{l}\right\}}^{\left\{n_{b}\right\}}(a, j) \prod_{l=1}^{b^{1}(\Sigma)} e^{-m_{l}\left(w_{l}+i a_{l}\right) / \alpha^{\prime}} \prod_{b=1}^{h^{1,1}(M)} e^{-n_{b} t_{b}}
\end{aligned}
$$

where $d_{\left\{m_{l}\right\}}^{\left\{n_{b}\right\}}(a, j)$ counts the number of holomorphic maps to discs $D \subset M$ which pass through $L^{a}$ at the insertion point of $V_{K}^{a}$ and $\beta_{j}$ at the insertion point of $V_{F}^{j}$, and which in addition have $[\partial D]=\sum_{l} m_{l} \gamma_{l}$ and $\left[D-\sum_{l} m_{l} D_{l}\right]=\sum_{b} n_{b} K_{b}$. 


\section{Discussion}

Space-filling D-branes wrapping supersymmetric cycles in Calabi-Yau manifolds provide one of the most natural classes of $\mathcal{N}=1$ supersymmetric models in string theory, and are attractive as concrete realizations of "brane world" scenarios. In this chapter, we have shown that the theories which arise from D6 branes wrapping supersymmetric three-cycles are in many ways analogous to heterotic $(0,2)$ models. In particular, although they are supersymmetric to all orders in $\alpha^{\prime}$, nonperturbative worldsheet effects can generate superpotentials and, perhaps, break supersymmetry.

These models differ from heterotic theories, however, in that mirror symmetry provides a dual description where the non-perturbative superpotential is computable at tree level in sigma model perturbation theory. This should be a powerful tool: most known dualities of $\mathcal{N}=1$ models, like heterotic/F-theory duality, relate instanton computations to other instanton computations (with worldsheet instantons mapping to euclidean wrapped branes of various sorts [35]). The present situation is considerably rosier, and it will be very interesting to exploit this to sum up instantons in this class of $\mathcal{N}=1$ string vacua.

The cases discussed in $\S 2.2$ (on the B-model side) should provide ideal test cases. In each case, one can realize (on a 5-brane wrapping a holomorphic curve) a theory with massless chiral fields, constrained by a higher order superpotential. The mirror D6 theory should provide us with an example of a brane wrapping a supersymmetric three-cycle $\Sigma$ with $b_{1}(\Sigma)>0$, but without a moduli space of the expected dimension. By the nonrenormalization theorem of $\S 2.1$, the moduli space on the A-model side must be lifted by a disc instanton generated superpotential. Work to explicitly construct the mirror cycles, and compute the relevant superpotentials, is under way [62]. Note that nonperturbative superpotentials which obstruct deformations of branes wrapped on three-cycles can resolve the puzzle for mirror symmetry raised by Thomas in [63]. On the other hand, we expect e.g. the supersymmetric $T^{3}$ used in [23] to derive mirror symmetry will survive instanton corrections. In the mirror picture this is obvious (since deformations of a point are unobstructed), and in the direct analysis presumably any holomorphic discs with boundary on the $T^{3}$ would come in families and cancel in their contribution to the superpotential.

In the regime where there are "small" holomorphic discs, new interesting phenomena should also occur. For instance, there are arguments in the mathematics 
literature that in some cases the classical moduli spaces of special Lagrangian threecycles will be manifolds with boundary (see $\S 5$ of [45]). This cannot be the case for physical applications of the sort we have discussed, involving wrapped branes in string theory. The moduli space (including Wilson line degrees of freedom) is that of a $4 \mathrm{~d} \mathcal{N}=1$ supersymmetric D-brane field theory. Assuming supersymmetry isn't broken, the quantum moduli space of supersymmetric ground states must be a Kähler manifold; there is no known dynamics that can create boundaries at codimension one in the moduli space of $4 \mathrm{~d} \mathcal{N}=1$ supersymmetric theories. The argument of [45] involves the fact that a holomorphic disc with boundary in the three-cycle is becoming very small; therefore, it is likely that some analogue of the phenomena discussed in [32] is occurring. Just as one can use theta angles to go around the boundaries of the classical Kähler cone and find intrinsically stringy Landau-Ginzburg phases of Calabi-Yau compactifications, it seems likely that one can use Wilson lines to go around the would-be boundary of moduli space discussed in [45] and find new, "quantum" supersymmetric three-cycles.

\section{Acknowledgements}

We would like to thank P. Aspinwall, D. Brace, H. Clemens, D.-E. Diaconescu, M. Douglas, S. Gukov, A. Klemm, G. Moore, D. Morrison, T. Pantev, R. Plesser, E. Silverstein and C. Vafa for interesting discussions about related subjects. This work was initiated at the Aspen Center for Physics. S. Kachru and A. Lawrence would like to acknowledge the kind hospitality of N. Seiberg and the Institute for Advanced Study while the bulk of this work was carried out. S. Kachru was supported in part by the generosity of the Ambrose Monell Foundation, an A.P. Sloan Foundation Fellowship and a DOE OJI Award; S. Katz was supported in part by NSA grants MDA904-98-1-0009 and MDA904-00-1-0052; A. Lawrence was supported in part by the DOE under contract DE-AC03-76F00515, and in part by the NSF via grant PHY94-07194 through the Institute for Theoretical Physics; and J. McGreevy was supported in part by the Department of Defense NDSEG Fellowship program. 


\section{Chapter 4: Mirror Symmetry for Open Strings}

We discuss the generation of superpotentials in $d=4, \mathcal{N}=1$ supersymmetric field theories arising from type IIA D6-branes wrapped on supersymmetric threecycles of a Calabi-Yau threefold. In general, nontrivial superpotentials arise from sums over disc instantons. We then find several examples of special Lagrangian three-cycles with nontrivial topology which are mirror to obstructed rational curves, conclusively demonstrating the existence of such instanton effects. In addition, we present explicit examples of disc instantons ending on the relevant three-cycles. Finally, we give a preliminary construction of a mirror map for the open string moduli, in a large-radius limit of the type IIA compactification.

\section{Introduction}

"The importance of instanton computations in string theory and in M-theory can hardly be overstated." [64]

- J.A. Harvey and G. Moore

There are many important motivations for studying the physics of D-branes on Calabi-Yau threefolds in type II string theories (or orientifolds thereof). To begin with, space-filling branes provide a microscopic construction of brane world models with $\mathcal{N}=1$ supersymmetry. In addition, physical objects in these theories (such as the moduli space of vacua and the superpotential) have a geometric expression. Hence, these theories provide a rich new context for studying quantum geometry via $\mathcal{N}=1$ field theories, along the lines of previous work on $\mathcal{N}=2$ brane probe theories [65]. For a fairly recent introductory review, see [66]; recent work on this subject has appeared e.g. in $[67,68,69,70,71,72,73,74,75,76,77,78,79]$.

The material in Chapter 3 appeared in "Mirror symmetry for open strings" with Shamit Kachru, Sheldon Katz, and Albion Lawrence [39] and is reprinted with permission of Phys. Rev. D. 
Consider a compactification of type IIA string theory on a Calabi-Yau threefold $M$. A single D6 brane wrapped on a supersymmetric three-cycle $\Sigma \subset M$ realizes a $4 \mathrm{~d} \mathcal{N}=1$ quantum field theory. ${ }^{13}$ In [69], we began to explore the consequences of mirror symmetry for such brane worldvolume theories (related work appears in $[80,81,82])$. We found that the moduli space of vacua has complex dimension $b_{1}(\Sigma)$, to all orders in $\sigma$-model perturbation theory. Any superpotential must be generated by nonperturbative worldsheet effects, i.e. disc instantons. Now, choose $\Sigma$ so that the mirror cycle $\mathcal{C}$ is a rational curve in the mirror threefold $W$. The mirror of the above D6-brane is a D5-brane wrapped on $\mathcal{C} \times \mathbb{R}^{4}$. When $\mathcal{C}$ has obstructed first-order deformations, the deformation is described by a massless scalar field with a higherorder superpotential; this superpotential is described exactly by classical geometry $[67,69]$. Mirror symmetry implies a disc instanton-generated superpotential for the IIA D6 brane. Ideally we could use this to compute the instanton sum exactly. The first obstacle to this program is that the explicit construction of such D-brane mirror pairs is quite difficult, and examples of compact special Lagrangian threecycles with $b_{1}(\Sigma) \neq 0$ have been scarce.

In this chapter, we further this program by providing examples of such pairs, and developing a preliminary understanding of the structure of the instanton sums and the mirror map. We begin in $\S 2$ with a more detailed review of supersymmetric D-branes in Calabi-Yau compactifications. These have a standard classification as A-type or B-type branes [83], which roughly correspond to special Lagrangian cycles and holomorphic cycles, respectively. The superpotentials on B-type branes arise from classical geometry $[67,69]$ and we review the geometry of a few specific examples (some with nontrivial superpotentials and some without). We also discuss the qualitative features of superpotentials for A type branes. In $\S 3$, we give an explicit construction of the special Lagrangian three cycles which are mirror to the explicit examples of B-type branes discussed in $\S 2$. In particular, we find examples of smooth three-cycles with nonvanishing $b_{1}$ whose mirrors have moduli space dimension less than $b_{1}$. This effectively proves the existence of disc instantongenerated superpotentials. We also give explicit examples of disc instantons, i.e.

13 To avoid RR tadpoles, one can take $M$ to be noncompact, or consider a full orientifold model which also has orientifold planes. Alternatively, since we will be working at tree level, one can consider a non-space filling brane whose worldvolume theory still has 4 supercharges, and view the superpotentials we compute in that context. 
holomorphic discs with boundary in a nontrivial homology class on the special Lagrangian cycle. In $\S 4$, we use mirror symmetry to make some statements about the instanton-generated superpotential for our A-type examples. We first discuss a mechanism by which disc instanton effects in our examples could (partially) cancel at special loci in closed string moduli space. We then give a preliminary description of the mirror map for open string moduli in one example. We close with a discussion of promising future directions in $\S 5$.

\section{Superpotentials from D-branes}

\subsection{A-type and B-type branes}

There are two distinct classes of supersymmetric branes in Calabi-Yau compactifications: A-type and B-type branes [83] (which can be constructed as boundary states in the topological A- and B-twisted sigma models respectively, following the notation of [84]). To help distinguish between these cases, we will denote by $M$ a Calabi-Yau used for studying A-type branes, and by $W$ a Calabi-Yau used for studying B-type branes. When we give examples in later sections, mirror pairs will be identified by using a common subscript, $\left(M_{i}, W_{i}\right)$. In geometric language, B-type branes correspond to branes wrapped on holomorphic 0,2,4 and 6-cycles of a CalabiYau $W$; while A type branes correspond to branes wrapped on a special Lagrangian three-cycle $\Sigma \subset M$. In both cases, one has to choose a gauge field configuration on the D-brane; the supersymmetry-preserving bundles correspond to flat bundles for A-type branes and to stable, holomorphic bundles for B-type branes. ${ }^{14}$

Assuming the branes to be space-filling, one can prove the following general results about the dependence of the $\mathcal{N}=1$ brane worldvolume action on the CalabiYau moduli:

- The superpotential for B-type branes depends only on the complex structure moduli, while FI terms depend only on Kähler moduli.

- The mirror story holds for A-type branes: the superpotential depends only on Kähler moduli, while the FI terms depend on complex structure moduli.

14 We are being schematic. A more precise discussion of B-type branes as coherent sheaves can be found in [85]; supersymmetric configurations with NS 2-form moduli turned on can be found in [86]. 
The statements about the superpotential were proven using worldsheet techniques in [67]. The correspondence between FI terms and Calabi-Yau moduli has been explored in $[66,1]$; explicit examples of superpotentials for B-type branes were given in $[67,69]$.

As with closed string $\sigma$-models, the open string $\sigma$-model coupling constants (in which one expands $\sigma$-model perturbation theory) are related to the choice of the Kähler form on $W$, and not to the complex structure. It follows that for Btype branes one can determine the superpotential exactly at $\sigma$-model tree level, using classical geometry. In contrast, for A-type branes (at least for a single brane, which is the case of interest to us here) the superpotential is entirely determined by "stringy" disc instanton corrections [69].

\subsection{Superpotentials for B-type branes}

Information about the superpotential for $B$-type branes is contained in the deformation theory for these branes (and for the gauge bundles on those branes). We will review here the case of branes wrapping curves in a threefold [67], since these are the examples we use in this chapter.

For a holomorphic curve $\mathcal{C}$ in a Calabi-Yau threefold $W$, the number of firstorder holomorphic deformations is $d=\operatorname{dim} H^{0}\left(\mathcal{C}, \mathcal{N}_{\mathcal{C}}\right)$, where $\mathcal{N}_{\mathcal{C}}$ is the normal bundle of $\mathcal{C} \subset W$. For a single D5-brane wrapping $\mathcal{C}$ in type IIB string theory, this leads to $d$ massless neutral chiral supermultiplets (in addition to the $U(1)$ vector multiplet). A superpotential for chiral multiplets naturally corresponds to an obstruction to extending the associated first-order deformations to higher order.

Geometrically, the obstruction can only arise if $H^{1}\left(\mathcal{C}, \mathcal{N}_{\mathcal{C}}\right)$ is nontrivial. For simplicity, we only consider a one-parameter deformation. If one chooses a small parameter $\epsilon$, and tries to find a finite holomorphic deformation order by order in $\epsilon$, one computes that the obstruction to finding a solution at each order is represented by an element of $H^{1}\left(\mathcal{C}, \mathcal{N}_{\mathcal{C}}\right)$ [87]. In particular, if there is a nonzero obstruction at order $\epsilon^{n}$, then this geometry is naturally described by a superpotential of the form $W=\Phi^{n+1} \cdot{ }^{15}$

15 Of course the correct normalization of the fields, and thus of the superpotential, depends on the Kähler metric, which we will not compute in this work. 
The simplest example of such obstructed curves begins with a threefold with $n$ isolated curves, as a particular deformation of the complex structure causes these curves to coincide. They become a single curve $\mathcal{C}$ of multiplicity $n$, and this curve has an obstruction at order $n$ to holomorphic deformations. Physically this is described by $n$ massive vacua coalescing into a single vacuum with superpotential $\Phi^{n+1} .{ }^{16}$

The B-type examples we will study realize this construction from the following starting point $[88,89]$. Begin with a Calabi-Yau threefold which contains a rational curve $\mathcal{C}$ fibered over a genus-g curve $\mathcal{S}_{g}$. One may canonically associate an element $\omega \in H^{1,0}\left(\mathcal{S}_{g}\right)$ with a non-toric ${ }^{17}$ first order deformation of the complex structure. The differential $\omega$ generically has $(2 g-2)$ simple zeros, which correspond to the isolated rational curves in the family $\mathcal{S}_{g}$ which survive the deformation. At points of positive codimension in complex moduli, these curves can coincide and form curves of higher multiplicity. ${ }^{18}$ There is a natural superpotential for a single D5brane wrapped on some fiber $\mathcal{C}_{z}$ over a point $z \in \mathcal{S}_{g}$, described in [69]. The local modulus $\phi$ of the rational curve $\mathcal{C}_{z}$ over $z$ can be thought of as an element of the holomorphic tangent space $T_{z}^{1,0} \mathcal{S}_{g}$, and it is the scalar component of a superfield $\Phi$. The superpotential is then:

$$
W(\Phi ; \omega)=\langle\omega, \Phi\rangle+\frac{1}{2 !}\langle\partial\langle\omega, \Phi\rangle, \Phi\rangle+\frac{1}{3 !}\langle\partial\langle\partial\langle\omega, \Phi\rangle, \Phi\rangle, \Phi\rangle+\cdots,
$$

evaluated at $z$. Here $\langle$,$\rangle is the usual inner product between forms and vectors, and$ $\partial$ is the Dolbeault operator on $\mathcal{S}_{g}$. It is easy to see that the expansion in (2.1) can be truncated after $(2 g-1)$ terms without changing the location and multiplicity of the critical points of $W$.

Below are several examples which realize this general framework. These will be our testing ground for a discussion of open-string mirror symmetry. The considerations of [69] yield some predictions for the mirror three-cycles which we will give

16 The situation is actually a bit more complicated than this. Our assertion only pertains to the case $\mathcal{N}_{\mathcal{C}}=\mathcal{O} \oplus \mathcal{O}(-2)$. It is an open problem to classify the possible superpotentials that can yield a single curve with multiplicity $n$, even in the next simplest case $\mathcal{N}_{\mathcal{C}}=$ $\mathcal{O}(1) \oplus \mathcal{O}(-3)$. An example in this case is the superpotential $W(\Phi, \Psi)=\Phi^{2} \Psi+\Psi^{3}$, corresponding to a curve with multiplicity 4 .

17 We will be studying hypersurfaces in weighted projective space: for these examples the non-toric complex structure deformations are those which are not monomial deformations of the defining equation.

18 In all of these cases, $\mathcal{N}_{\mathcal{C}}=\mathcal{O} \oplus \mathcal{O}(-2)$, so our previous discussion applies. 
at the end of these examples: we will describe and explore the mirror examples in $\S 3$.

\section{Ur-Example}

Our examples will all be orbifolds of the Calabi-Yau hypersurface $W$ of degree 8 in $\mathbb{P}_{1,1,2,2,2}^{4}$, defined for example by the equation

$$
p=z_{1}^{8}+z_{2}^{8}+z_{3}^{4}+z_{4}^{4}+z_{5}^{4}=0 .
$$

$W$ has a singularity at $z_{1}=z_{2}=0$, inherited from the ambient weighted projective space. Blowing up this $Z_{2}$ singularity yields a family of $\mathbb{P}^{1} \mathrm{~s}$, parametrized by the genus 3 curve $\mathcal{S}_{3}$ :

$$
z_{3}^{4}+z_{4}^{4}+z_{5}^{4}=0
$$

The non-toric deformations associated to $H^{(1,0)}\left(\mathcal{S}_{3}\right)$ generically lift the family $\mathcal{S}_{3}$ of $\mathbb{P}^{1} \mathrm{~s}$, leaving four isolated $\mathbb{P}^{1} \mathrm{~s}$.

One can see the non-toric deformations explicitly, by considering an equivalent description of $W$ as a complete intersection of a quartic and a quadric in $\mathbb{C P}^{5}$ following [88]. One sees the equivalence by setting the homogeneous coordinates $\left(y_{0}, \cdots, y_{5}\right)$ of $\mathbb{C P}^{5}$ equal to $\left(z_{1}^{2}, z_{2}^{2}, z_{1} z_{2}, z_{3}, z_{4}, z_{5}\right)$. Then the quadric equation

$$
y_{2}^{2}=y_{0} y_{1}
$$

of rank three is automatically satisfied. The model in $\mathbb{P}^{5}$ obviously has complex structure moduli which deform (2.4) to an equation of higher rank. If one deforms the quadric to have rank four or fewer, then one is still describing points in the complex structure moduli space of $W .{ }^{19}$ Deformations to quadrics of rank greater than four correspond to making an extremal transition from $W$ to another CalabiYau space. One finds a three-dimensional space of deformations of the quadric which leave one in the moduli space of $W$, hence there are three non-toric deformations. This story is described in full generality (in the case of a family of $\mathbb{P}^{1}$ s parameterized by a genus $g$ curve, and the corresponding $g$ non-toric deformations) in [89].

19 To see this, note that both (2.4) and the rank 4 quadric $y_{0} y_{1}=y_{2} y_{3}$ can be desingularized by the same blowup $y_{0}=y_{2}=0$, hence both blowups fit into the same moduli space. 
It is evident that when one deforms (2.4) by a term which increases the rank to four, e.g. $y_{3}^{2}$, one destroys the family of $\mathbb{P}^{1}$ s. This is because the family is located at $y_{0}=y_{1}=y_{2}=0$ (which is the same as $z_{1}=z_{2}=0$ ); the addition of $y_{3}^{2}$ to (2.4) would then force $z_{3}=0$ also. But then the former genus 3 curve (2.3) collapses to the four points $z_{4}^{4}+z_{5}^{4}=0$. Hence, instead of a one-parameter family there are now 4 isolated $\mathbb{P}^{1} \mathrm{~s}$.

Upon wrapping a single D5-brane on a member of the family of $\mathbb{P}^{1} \mathrm{~s}$ one finds a $U(1)$ gauge theory in four dimensions with a single neutral chiral multiplet $\phi$ parameterizing a local neighborhood in $\mathcal{S}_{3}$. After a generic non-toric deformation described by $\omega,(2.1)$ will describe a superpotential with four massive vacua.

In the mirror manifold, $M$, the non-toric deformations have the following description. Toric Kähler moduli in weighted projective space arise from the volume of the space, the blow-up parameters for the fixed loci of the Greene-Plesser orbifold group, and blow-up parameters for any singularities of the weighted projective space which intersect the Calabi-Yau. If the CY hypersurface intersects one of these loci $n+1$ times, the toric Kähler deformation changes the size of all $n+1$ divisors simultaneously, while the remaining $n$ "non-toric" moduli change the relative sizes.

In our examples, mirror symmetry demands the following statement about the three-cycles $\Sigma \subset M$ mirror to $\mathcal{C} \subset W$. At the locus in Kähler moduli space with the three non-toric moduli turned off, we have a unique first-order deformation which by $[90,69]$ requires $b_{1}(\Sigma) \geq 1$. (If the inequality is not saturated, the instanton sum must give $b_{1}-1$ chiral multiplets a mass.) The instanton-generated superpotential for one of these moduli must vanish until the non-toric deformations are turned on, and then the moduli space generically splits into four massive vacua.

\section{Example I}

The first example which we will discuss is $W_{1}$, the orbifold of $W$ and $\mathcal{S}_{3} \subset W$ by the discrete $Z_{4} \times Z_{2} \times Z_{2}$ group with generators

$$
(1, i, i, i, i), \quad(1,1,-1,-1,1), \quad(1,1,1,-1,-1) .
$$

The family of $\mathbb{P}^{1} \mathrm{~s}$ on $W$ located at $z_{1}=z_{2}=0$ is orbifolded by (2.5), and the curve (2.3) becomes a genus 0 curve after orbifolding. Because $\mathbb{P}^{1}$ has no holomorphic one forms, this model does not admit non-toric deformations which destroy the family 
of holomorphic spheres. Therefore, there is never a nontrivial superpotential, for any complex structure.

Mirror symmetry requires that $M_{3}$ has a one-parameter family of supersymmetric three-cycles. This would be most simply realized by a family of three-cycles $\Sigma$ with $b_{1}(\Sigma)=1$. No Kähler deformation of $M_{3}$ should lead to a nontrivial disc instanton generated superpotential, so the family of three cycles survives quantum corrections even after deformations of closed string Kähler moduli.

If $M_{1}$ is the mirror of $W_{1}$, we will see in Appendix A that the mirror cycles $\Sigma \subset M_{1}$ to our family of $\mathbb{P}^{1}$ s have $b_{1}(\Sigma)>1$. Therefore we expect disc instanton effects to give masses to $b_{1}(\Sigma)-1$ of the moduli of $\Sigma$ predicted by the classical geometry.

\section{Example II}

Our next example, $W_{2}$, arises from orbifolding $W$ and $\mathcal{S}_{3} \subset W$ by the $Z_{4} \times Z_{4}$ discrete group with generators

$$
(1, i, i, 1,-1), \quad(1,1,1, i,-i)
$$

Once again we have a family of $\mathbb{P}^{1} \mathrm{~s}$ at $z_{1}=z_{2}=0$. The curve $(2.3)$ becomes a genus-1 curve $\mathcal{S}_{1}$, after orbifolding by (2.6). Thus, if we wrap a D5-brane around a member of this family, there is a single parameter in the superpotential $W$ associated to the holomorphic differential on the curve $\mathcal{S}_{1}$. The corresponding superpotential (2.1) is just

$$
W(\Phi)=c \Phi
$$

where $c$ is related to the magnitude of the non-toric blowup. When $c \neq 0$ there are no supersymmetric vacua: the auxiliary field $F$ in the chiral multiplet is nonvanishing, and since we are coupled to closed string theory the $4 \mathrm{~d}$ gravitino gains a mass. This is in keeping with the fact that after the deformation, the holomorphic spheres have all disappeared.

In the absence of coupling to gravity, one can redefine the supercharges so that the superpotential (2.7) does not break supersymmetry; it simply adds a harmless constant to the Lagrangian. This is reflected clearly in the geometry of the example. In a local neighborhood of the $g=1$ curve of $\mathbb{P}^{1} \mathrm{~s}$ in $W_{2}$, the manifold looks like a product of an $A_{1}$ ALE space and a $T^{2}$. This local geometry is hyperkähler and so 
has a family of complex structures, parametrized by an $S^{2}$. Upon performing the non-toric deformation (2.7), one can choose a different complex structure so that there are still holomorphic curves. Since $W_{2}$ is not hyperkähler, this is prevented by the global geometry at finite volume. Hence, global features of $W_{2}$ are important in determining that supersymmetry is broken, a fact which clearly reflects the need to couple the D-brane worldvolume theory to gravity in order to diagnose the supersymmetry breaking.

If $M_{2}$ is the mirror of $W_{2}$, the mirror cycles $\Sigma \subset M_{2}$ to our family of $\mathbb{P}^{1} \mathrm{~s}$ should also live in a one-dimensional family, so that $b_{1}(\Sigma) \geq 1$. The non-toric Kähler deformation of $M_{2}$ breaks supersymmetry entirely via disc instanton effects.

\section{Example III}

Example III works much like Example I. Let $M_{3}$ be the orbifold of $W$ by the $Z_{2}$ symmetry generated by $\tilde{g}$ :

$$
\tilde{g}=(1,1,1,-1,-1)
$$

We will consider B-type branes on the mirror $W_{3}$ of $M_{3}$. In $W_{3}$ there is still a oneparameter family of $\mathbb{P}^{1} \mathrm{~s}$, parametrized by a $\mathbb{P}^{1}$ (roughly obtained by orbifolding the genus 3 curve in $W$ ). Again, there is no superpotential for this modulus for any value of the complex structure.

For the mirror A-cycle we will find below that $b_{1}(\Sigma)=1$, so classical results apply for all values of the Kähler moduli of $M_{3}$.

\subsection{Qualitative features of superpotentials for A-type branes}

Coordinates on the moduli space of A-type branes

Let $M$ be a general Calabi-Yau threefold, and $\Sigma \subset M$ a special Lagrangian three-cycle. For simplicity, assume $b_{1}(\Sigma)=1$, and assume there is a single holomorphic disc instanton $D$ bounded by a representative $\gamma$ of the generating class in $H_{1}(\Sigma)$. The cycle $\Sigma$ moves in a one-dimensional family in $\sigma$-model perturbation theory; as discussed in $[81,69]$, we can parameterize this family locally by a modulus field $\phi$ :

$$
\phi=\operatorname{Area}(D)+i a
$$

where the area is measured in string units, and $a$ is an axion (the Wilson line of the brane $U(1)$ gauge field around $\gamma$ ). 
This was the picture given in [69], but a moment's thought indicates that it should be modified. Consider for example a special Lagrangian torus in $T^{6}$. There are clearly no holomorphic discs bounding the cycles of $T^{6}$, but this does not mean that there is no moduli space for the special Lagrangian subcycle. Indeed there is a simple ansatz which naturally generalizes the above. Begin with a reference three-cycle $\Sigma_{0}$ in some family $\Sigma_{t}$, defined by an embedding $f_{t}: \Sigma_{0} \hookrightarrow M$. Choose a family of deformations constructed from a harmonic form in some class in $H^{1}\left(\Sigma_{0}\right)$ [90]. Then choose some one-cycle $\gamma_{0} \in \Sigma_{0}$ whose class in $H_{1}\left(\Sigma_{0}\right)$ is dual to this cohomology class via the metric. As $t$ varies in the chosen family of deformations, $f_{t}(\gamma)$ will sweep out some tube $T$ in $M$. A natural coordinate $\phi$ is:

$$
\phi=\int_{T} \omega+i \int_{\gamma_{t}} A_{t}
$$

where $\omega$ is the Kähler form on $M$ and $A_{t}$ is a flat connection on $\Sigma_{t}$. When the tube is holomorphic, the real part is simply the area of the tube.

\section{Finding nontrivial superpotentials}

Before launching into a detailed discussion of specific three-cycles mirror to the above examples, we would like to gain some general and intuitive understanding of the form of the superpotentials directly in the language of the three-cycle geometry.

Following [82], the sum over multiple covers of $D$ yields a superpotential

$$
W= \pm \sum_{n=1}^{\infty} \frac{e^{-n \phi}}{n^{2}}
$$

The sign here depends on details of the fermion determinants around the instanton solution [80]. It follows from (2.11) that

$$
\frac{\partial W}{\partial \phi} \sim \pm \sum_{n=1}^{\infty} \frac{e^{-n \phi}}{n}=\mp \log \left(1-e^{-\phi}\right) .
$$

This has a single critical point at $\phi=\infty$, which from (2.9) is the open string analogue of large radius. Of course one expects that to reach $\phi=\infty, M$ must be at some infinite-radius point.

Now, suppose instead that we have $k$ disc instantons $D_{i}$ bounding the same homology class $\gamma \in H_{1}(\Sigma)$. $D_{i}$ may differ by homology classes in $M$. Choose $\operatorname{Re}(\phi)$ to be the area of $D_{1}$. The exponential of the action for instanton $D_{i}$ is that for $D_{1}$ 
times a factor $q_{i}$, the exponential of the (complexified) volume of $\left[D_{i}-D_{1}\right] \in H_{2}(M)$. Finally, assume each of these discs are isolated. The resulting superpotential is:

$$
W=\sum_{i=1}^{k} \sum_{n=1}^{\infty} \sigma_{i} \frac{e^{-n \phi}}{n^{2}} q_{i}^{n},
$$

where $\sigma_{i}$ is the sign of the $\sigma$-model fermion determinant for the instanton $D_{i}$. The supersymmetric vacua satisfy:

$$
\sum_{i=1}^{k} \sigma_{i} \log \left(1-q_{i} e^{-\phi}\right)=0 .
$$

This is equivalent to a polynomial equation in $e^{-\phi}$, whose degree is the greater of $\#\left\{i \mid \sigma_{i}=1\right\}$ and $\#\left\{i \mid \sigma_{i}=-1\right\}$. Note that the critical points need not all be at large radius. The precise locations of the critical points depend on the closed string Kähler moduli through the $q_{i}$.

We get similar results when we include new disc instantons in the class $d \gamma$ for varying $d$. The general result is that if we have $k_{i}$ disc instantons in classes $d_{i} \gamma$, then (assuming for simplicity that all the fermion determinants are positive) there are $\sum k_{i} d_{i}$ supersymmetric vacua. For families of discs some open-string version of the Gromov-Witten invariants of closed string instantons should replace $k_{i}$.

The main point of this discussion is that it is not difficult to imagine oneparameter families of special Lagrangian manifolds which yield, after disc instanton corrections, a discrete set of supersymmetric vacua. This is fortunate as the mirrors of the B-brane configurations discussed in $\S 2.2$ must exhibit this behaviour.

Another lesson is that string instanton effects alter our expectations of the topology of our three-cycles. The natural physical measure of $b_{1}(\Sigma)$ within $\sigma$-model perturbation theory is the number of massless chiral multiplets for a single D6brane wrapped on this cycle. However, disc instanton effects may well give some of these chiral multiplets a mass, in which case there is no obvious physical distinction between the original three-cycle and a cycle with smaller $b_{1}$.

\section{Special features of A-cycles arising as real slices}

We will be focusing on special Lagrangian three-cycles constructed as the fixed point locus of antiholomorphic involutions acting on $M$, in other words antiholomorphic maps $\sigma: z \rightarrow \bar{z}$ which square to the identity. The standard example, 
which we will use in every case, is the real slice arising as the fixed point set of $z_{i} \rightarrow \bar{z}_{i}$. In this case, for each holomorphic disc we get a conjugate holomorphic disc. If $f: D \rightarrow M$ is a holomorphic map, we can define its conjugate holomorphic disc $g: D \rightarrow M$ by $g(z)=\overline{f(\bar{z})}$. Upon gluing these discs together we find that for any special Lagrangian submanifolds obtained as fixed points of antiholomorphic involutions, holomorphic disc instantons always come as two halves of a rational curve in $M$. Furthermore, it is natural to conjecture that as this special Lagrangian cycle moves through a family $\Sigma_{t}$, one may find a set of one-cycles $\gamma_{t} \in \Sigma_{t}$ whose images in $M$ sweep out this rational curve.

The superpotential can be derived from a variant of (2.13). Let $\mathbb{P}$ be the rational curve in question, and $t$ be the integral of the complexified Kähler form of $M$ over $\mathbb{P}$. Let $z$ be the action of the instanton described by $f$; the action for the instanton described by $g$ is then $t-z$. Assuming the fluctuation determinants have the same sign, the superpotential one gets from summing over multiple covers is up to overall sign:

$$
W=\operatorname{Li}_{2}\left(1-e^{-z}\right)+\operatorname{Li}_{2}\left(1-e^{-t+z}\right)
$$

It is easy to see that this has a supersymmetric vacuum at $z=t / 2$. At this point in the open- and closed string moduli space the superpotential is that of the local model in [82].

\section{Constructing the mirror three-cycles}

The next step to fleshing out the mirror map for open strings is, of course, to characterize the mirror map for the submanifolds on which they end. In this section we will find explicit special Lagrangian three-cycles mirror to elements of the families of $\mathbb{P}^{1}$ s described in the examples above.

\subsection{Strategy for identifying mirror cycles}

At an arbitrary point in the closed string moduli space, it will be fairly difficult to find explicit mirror cycles. Instead we focus on loci of the moduli space with physical and mathematical significance. In our B-cycle examples, the family of $\mathbb{P}^{1}$ s around which we intend to wrap D5-branes are known to have zero volume at some submanifold in the full (complex and Kähler) moduli space; these points 
occur when the resolutions of the orbifold singularities discussed above have been turned off (along with the associated NS-NS 2-form moduli). We may identify these points physically by studying BPS D2-branes wrapped on the same cycles in type IIA string theory. ${ }^{20}$ These D2-branes form massless (vector) multiplets at this discriminant locus as guaranteed by the BPS formula.

In type IIB on the mirror CY, the BPS formula implies that a wrapped D3brane must become massless at the mirror discriminant locus. Since the mass receives no closed string worldsheet instanton corrections, we need simply find the mirror discriminant locus and the vanishing three-cycle via classical geometry. We will discuss the identification of this pair of cycles in $W \subset \mathbb{P}_{1,1,2,2,2}^{4}$ and its mirror; the same logic leads to a similar identification in all of our examples.

The mirror manifold $M$ of $W$ is easily constructed using the Greene-Plesser construction [91]. One quotients $W$ by a suitable maximal group of scaling symmetries, leaving only two complex structure deformations of $M$. These can be represented by the coefficients of the monomials $z_{1} \cdots z_{5}$ and $z_{1}^{4} z_{2}^{4}$ in the defining equation for $M$.

Let us work on the locus in moduli space where the defining equation is:

$$
\left(z_{1}^{4}-z_{2}^{4}\right)^{2}-2 \epsilon z_{1}^{4} z_{2}^{4}+z_{3}^{4}+z_{4}^{4}+z_{5}^{4}=0
$$

Here we have set the coefficient of $z_{1} \cdots z_{5}$ to zero; this subspace of the complex structure moduli space of $Y$ intersects the discriminant locus at $\{\epsilon=0, \epsilon=$ $-2\}$. These two points in moduli space can be seen to determine the same CY manifold by redefining $z_{2}$ by an eighth root of unity. Now, we want to construct a supersymmetric three-cycle which is mirror to a member of the family of $\mathbb{P}^{1} \mathrm{~s}$ on $W$, discussed in $\S 2.2$. At least near large complex structure, $\delta z_{1} \cdots z_{5}$ is the complex deformation of $M$ mirror to the size of the projective space $W \mathbb{P}_{1,1,2,2,2}^{4}$. Furthermore, we can identify $\epsilon$ as mirror to the modulus controlling the size of the exceptional $\mathbb{P}^{1}$ in $W$, as explained in [88]. Therefore, we are looking for a threecycle $\Sigma \subset M$ which collapses as $\epsilon \rightarrow 0$. This identification of mirror moduli holds in the other cases as well. We will find a particular such three-cycle $\Sigma$ as a component of the fixed point locus of a real involution acting on the A-model CY. Some set of

20 All of our statements are at string tree level so we can be cavalier about changing brane dimension like this. 
fixed points of the Greene-Plesser quotient intersect the three-cycle, and the details of the resolution of these singularities will determine the topology of $\Sigma$.

In the following subsections we study the mirrors of the examples considered in $\S 2$. Mirror symmetry reverses the order of increasing complexity, so we will examine the three examples in reverse order.

\subsection{Example III}

In this example the three-cycle topology is the simplest, since the fewest blowups are required. Recall that $M_{3}$ is the orbifold of $W$ by $\tilde{g}=(1,1,1,-1,-1)$. The only fixed points are at $z_{4}=z_{5}=0$, so we introduce a second $\mathbb{C}^{*}$ action and a new coordinate $z_{6}$, where the second $\mathbb{C}^{*}$ acts by $\left(z_{4}, z_{5}, z_{6}\right) \rightarrow\left(\lambda z_{4}, \lambda z_{5}, \lambda^{-2} z_{6}\right)$. The defining equation is modified to:

$$
\left(z_{1}^{4}-z_{2}^{4}\right)^{2}-2 \epsilon z_{1}^{4} z_{2}^{4}+z_{3}^{4}+z_{6}^{2}\left(z_{4}^{4}+z_{5}^{4}\right)=0
$$

so that the manifold is preserved by this second $\mathbb{C}^{*}$. Now consider the real involution:

$$
\sigma:\left(z_{1}, \cdots, z_{6}\right) \rightarrow\left(\bar{z}_{1}, \cdots, \bar{z}_{6}\right)
$$

We obtain a three-cycle $N$ as the fixed point locus of $\sigma$ ( $N$ has two components, which are basically two copies of the desired three-cycle $\Sigma$ ). We will see below that it vanishes as $\epsilon \rightarrow 0$. For the rest of this subsection, we take $z_{1}, \cdots, z_{6}$ to be real (since we wish to work on the fixed point locus of $\sigma$ ).

We use the $\mathbb{C}^{*}$ action of the $\mathbb{P}^{4}$ to set $z_{2}=1$; we will see presently that we will not need to leave this coordinate patch. Furthermore, we define:

$$
x=z_{1}^{4}, \quad Q=z_{3}^{4}+z_{6}^{2}\left(z_{4}^{4}+z_{5}^{4}\right), \quad A=2 \epsilon+\epsilon^{2} .
$$

Solving (3.2) for $x$ in terms of the remaining variables we find:

$$
x=1+\epsilon \pm \sqrt{A-Q} .
$$

We have two branches of solutions for $x$, which meet when $Q=A$. The real slice includes only the region $A \geq Q$, since otherwise $x=z_{1}^{4}$ would be imaginary. 
Next, we blow up the orbifold singularity induced by $\tilde{g}$ using the language of symplectic quotients. We introduce a new Kähler parameter $r$ in the following "D-term equation" (c.f. [92]):

$$
\left|z_{4}\right|^{2}+\left|z_{5}\right|^{2}-2\left|z_{6}\right|^{2}=r
$$

in the description of the full complex manifold $M$ : along the real locus we can dispense with the absolute values. Note that in the full CY we have to gauge away the $U(1)$ under which $\left(z_{4}, z_{5}, z_{6}\right)$ have charges $(1,1,-2)$. Since the $z_{i}$ are real on $N$, the only gauge transformation which acts as an identification on $N$ is $\left(z_{4}, z_{5}, z_{6}\right) \mapsto\left(-z_{4},-z_{5}, z_{6}\right)$, i.e. the orbifold by $\tilde{g}$.

Next, we can solve (3.5) for $z_{4}$ :

$$
z_{4}= \pm \sqrt{r-z_{5}^{2}+2 z_{6}^{2}} .
$$

This gives two branches for $z_{4}$ which are glued together along the hyperbola $z_{5}^{2}-$ $2 z_{6}^{2}=r$. Reality of $z_{4}$ requires that $r \geq z_{5}^{2}-2 z_{6}^{2}$. These conditions bound $z_{3}^{2}, z_{5}^{2}, z_{6}^{2}$ from above, allowing us to stay on the patch where $z_{2}=1$.

Consider the regime $0<\epsilon<<1, r>\epsilon$. The region $Q \leq A$ intersects the region of real $z_{4}$ in a region with the topology of a solid cylinder, as pictured below.

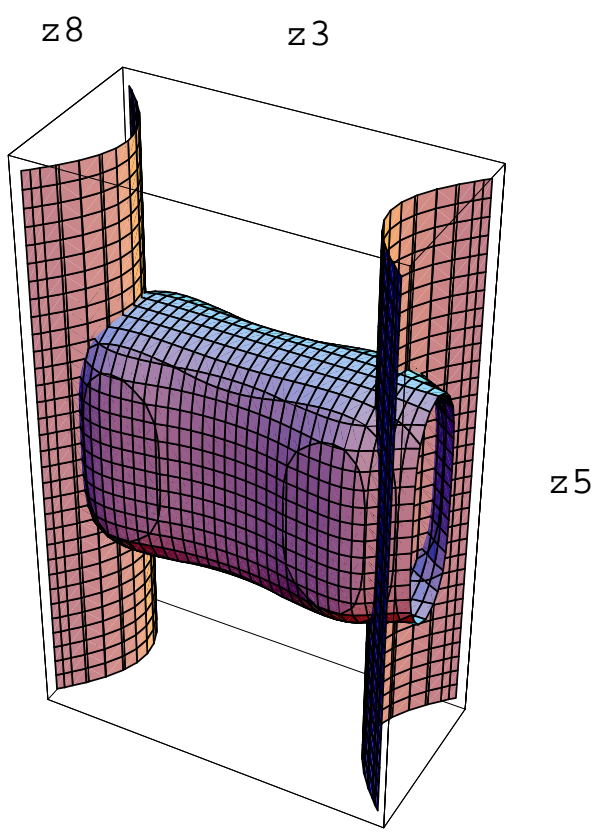

Fig. 1: $z_{4}$ is real between the walls; $x$ is real inside the tube. 
Making the orbifold identification merely halves the circumference of this cylinder. The two branches of solutions for $z_{4}$ and the two branches of solutions for $z_{1}^{4}$ give rise to four copies of this cylinder, which are glued along the loci where the $z_{4}$ branches meet and the $z_{1}$ branches meet (the sheet of hyperbolas and $Q=A$ respectively). The gluing along the boundaries of the $z_{4}$ branches yields two solid tori; gluing along the boundaries of the $z_{1}$ branches then yields the closed threemanifold $\Sigma \sim S^{2} \times S^{1}$, which has $b_{1}(\Sigma)=1$. Note that $N$ consists of two copies of $\Sigma$, one with $z_{1}>0$, and the other with $z_{1}<0$ (we will abuse notation and call both copies by the same name, since in any case they are identical).

Because $\Sigma$ is a smooth special Lagrangian three-cycle with $b_{1}=1$, it is guaranteed by McLean's theorem to come in a family of special Lagrangian cycles of real dimension one [90]. Since $Q$ is positive semidefinite on the real slice, when $\epsilon \rightarrow 0$ the locus $Q \leq A$ collapses and the two components of $N$ are no longer finite-volume three-manifolds. We identify the two components of $N$ with two members of the family of $\mathbb{P}^{1} \mathrm{~s}$ in $W_{3}$.

A D6 brane wrapping $\Sigma$ would naively yield, in the transverse $3+1$ dimensions, a $4 \mathrm{~d} \mathcal{N}=1$ field theory with $U(1)$ gauge group and a single neutral chiral multiplet $\phi$. Although $\phi$ has no superpotential to all orders in sigma model perturbation theory (this is the string theory analog of McLean's theorem), $\phi$ can receive a superpotential from disc instantons [69]. In this case, we know from the mirror B-model geometry that there are no non-toric deformations which would lift the moduli space of supersymmetric $\mathbb{P}^{1} \mathrm{~s}$. This implies that there is no disc-generated superpotential in this case.

\section{Disc Instantons}

Some explicit examples of disc instantons with boundary on $\Sigma$ can be constructed in this example. Consider the upper half plane parametrized by $u$. Let $z_{1}, \cdots, z_{6}$ be given by

$$
\left(z_{1}, \cdots, z_{6}\right)=\left(a_{1} u, a_{2} u, a_{3} u^{2}, 1,1,0\right)
$$

We take the $a_{i}$ to be real; this guarantees that the boundary of the disc (where $u$ is real) is mapped to $\Sigma$.

The disc must lie in $M_{3}$, which means that:

$$
\left(a_{1}^{4}-a_{2}^{4}\right)^{2}-2 \epsilon a_{1}^{4} a_{2}^{4}+a_{3}^{4}=0 .
$$


Solutions to (3.8) provide holomorphic maps into $M_{3}$ with boundary on $\Sigma$. In fact this ansatz yields a one-parameter family of discs: the constraint (3.8) eliminates one of the $a_{i}$ and the freedom to rescale the $u$ plane fixes another, but there is one free parameter left in the ansatz. The fact that mirror symmetry implies that there is no disc-generated potential in this case suggests that there is a cancellation between the contributions of different discs. We will discuss such a mechanism in $\S 4$.

\subsection{Example II}

The mirror $M_{2}$ of $W_{2}$ is constructed by orbifolding $W$ by the $Z_{4}$ group generated by $g=(1,1,-1, i, i)$. The group element $g^{2}=(1,1,1,-1,-1)$ is the symmetry by which we orbifolded in Example III, so we should still perform the resolution above. However, $g$ itself fixes the locus $z_{3}=z_{4}=z_{5}=0$, which must be independently blown up. This is achieved by introducing another variable, $z_{7}$, and another $\mathbb{C}^{*}$ action - the charges are summarized in the following table:

$\begin{array}{cccccccc} & z_{1} & z_{2} & z_{3} & z_{4} & z_{5} & z_{6} & z_{7} \\ \mathbb{C}_{1}^{*} & 1 & 1 & 2 & 2 & 2 & 0 & 0 \\ \mathbb{C}_{2}^{*} & 0 & 0 & 0 & 1 & 1 & -2 & 0 \\ \mathbb{C}_{3}^{*} & 0 & 0 & 2 & 1 & 1 & 0 & -4 .\end{array}$

The defining equation is modified to

$$
\left(z_{1}^{4}-z_{2}^{4}\right)^{2}-2 \epsilon z_{1}^{4} z_{2}^{4}+z_{7}^{2} z_{3}^{4}+z_{7} z_{6}^{2}\left(z_{4}^{4}+z_{5}^{4}\right)=0
$$

We will use $z_{3}, z_{5}$, and $z_{6}$ as coordinates on the real slice, $N$, which is the fixed point locus of

$$
\sigma:\left(z_{1}, \cdots, z_{7}\right) \rightarrow\left(\bar{z}_{1}, \cdots, \bar{z}_{7}\right)
$$

Redefining

$$
x=z_{1}^{4}, \quad Q=z_{7}^{2} z_{3}^{4}+z_{7} z_{6}^{2}\left(z_{4}^{4}+z_{5}^{4}\right), \quad A=2 \epsilon+\epsilon^{2}
$$

we find:

$$
x=z_{2}^{4}(1+\epsilon \pm \sqrt{A-Q}) .
$$

On the real slice, the D-term equations for $\mathbb{C}_{2,3}^{*}$ read

$$
z_{4}^{2}+z_{5}^{2}-2 z_{6}^{2}-r_{2}=0,2 z_{3}^{2}+z_{4}^{2}+z_{5}^{2}-4 z_{7}^{2}-r_{3}=0
$$


Solving (3.12) for $z_{4}$ and $z_{7}$ we find:

$$
z_{4}= \pm \sqrt{r_{2}-z_{5}^{2}+2 z_{6}^{2}}
$$

and

$$
z_{7}= \pm \sqrt{\frac{1}{2}\left(r_{2}-r_{3}+z_{3}^{2}+z_{6}^{2}\right)} .
$$

If we choose Kähler moduli so that $r_{2}>r_{3}$, then $z_{7}$ never vanishes, and the two branches of solutions never meet. On the branch where $z_{7}>0, Q=z_{7}^{2} z_{3}^{4}+z_{7} z_{6}^{2}\left(z_{4}^{4}+\right.$ $\left.z_{5}^{4}\right)$ is positive semidefinite as in $\S 3.2$, and (3.11) tells us that this component of the real slice vanishes as $\epsilon \rightarrow 0$. The other component of the real slice does not shrink on this locus and so is of no interest to us. Under the restriction that $z_{4}= \pm \sqrt{r_{2}-z_{5}^{2}+2 z_{6}^{2}}$ is real, the three-cycle of interest again resides on the patch where $z_{2}=1$ (for this regime in closed string moduli space). The determination of topology goes through in complete analogy with Example III, and we again find two components of the real slice $N$, each of which is topologically $S^{2} \times S^{1}$. We again call the components $\Sigma$.

So we see again that a D6-brane on $\Sigma$ has a one-dimensional moduli space to all orders in $\sigma$-model perturbation theory. The non-toric deformation of $W_{2}$ which lifts the moduli space of supersymmetric $\mathbb{P}^{1} \mathrm{~s}$ must in fact map to a small deformation of the Kähler structure of $M_{2}$. This deformation cannot change the topology of $\Sigma$, since $\Sigma$ is a smooth three-cycle and the deformation can be made arbitrarily small. Hence, for the moduli spaces of the mirror pair to match, the non-toric Kähler deformation must activate a disc-generated superpotential. We give further evidence for this below.

\section{Disc Instantons}

For this example we can again construct explicit examples of disc instantons with boundary on $\Sigma$. Using the holomorphic quotient description of $M_{2}$, we fix the three $\mathbb{C}^{*}$ actions to set $z_{4}=z_{5}=z_{7}=1$.

Consider the upper half plane parametrized by $u$. Let $z_{1}, \cdots, z_{7}$ be given by

$$
\left(z_{1}, \cdots, z_{7}\right)=\left(a_{1} u, a_{2} u, a_{3} u^{2}, 1,1,0,1\right)
$$

Again the $a_{i}$ are real, so that the boundary of the disc $u \in \mathbb{R}$ is mapped to $\Sigma$.

In order that the disc lies in $M_{2}, a_{1,2,3}$ must again satisfy Eq. (3.8). As before, we find a one-parameter family of holomorphic maps of the disc into $M_{2}$ with boundary on $\Sigma$. 


\subsection{Example $I$}

The mirror $M_{1}$ of $W_{1}$ is constructed by orbifolding $W$ by the $Z_{2} \times Z_{2}$ group generated by $g_{1}=(1,1,-1,-1,1)$ and $g_{2}=(1,1,1,-1,-1)$. This example provides the richest spectrum of phenomena for the A-cycles in $M_{1}$ as we have to perform the most blowups. Augmenting the weighted projective space by the following additional variables and $\mathbb{C}^{*}$ actions allows us to resolve all singularities which intersect the three-cycle: 


$\begin{array}{lcccccccc} & z_{1} & z_{2} & z_{3} & z_{4} & z_{5} & z_{6} & z_{7} & z_{8} \\ \mathbb{C}_{1}^{*} & 1 & 1 & 2 & 2 & 2 & 0 & 0 & 0 \\ \mathbb{C}_{2}^{*} & 0 & 0 & 0 & 1 & 1 & -2 & 0 & 0 \\ \mathbb{C}_{3}^{*} & 0 & 0 & 1 & 0 & 1 & 0 & -2 & 0 \\ \mathbb{C}_{4}^{*} & 0 & 0 & 1 & 1 & 0 & 0 & 0 & -2 .\end{array}$

The defining equation for $M_{1}$ is:

$$
\begin{array}{r}
0=\left(z_{1}^{4}-z_{2}^{4}\right)^{2}-2 \epsilon z_{1}^{4} z_{2}^{4}+z_{7}^{2} z_{8}^{2} z_{3}^{4}+z_{6}^{2} z_{8}^{2} z_{4}^{4}+z_{6}^{2} z_{7}^{2} z_{5}^{4} \\
\equiv\left(z_{1}^{4}-z_{2}^{4}\right)^{2}-2 \epsilon z_{1}^{4} z_{2}^{4}+Q
\end{array}
$$

We will use $z_{3}, z_{4}$ and $z_{5}$ as our independent variables. Solving the D-term equations associated to $\mathbb{C}_{2}^{*}, \mathbb{C}_{3}^{*}$ and $\mathbb{C}_{4}^{*}$ for $z_{6}, z_{7}$ and $z_{8}$ on the real slice gives

$$
\begin{aligned}
z_{6} & = \pm \sqrt{\frac{1}{2}\left(-r_{2}+z_{4}^{2}+z_{5}^{2}\right)} \\
z_{7} & = \pm \sqrt{\frac{1}{2}\left(-r_{3}+z_{3}^{2}+z_{5}^{2}\right)} \\
z_{8} & = \pm \sqrt{\frac{1}{2}\left(-r_{4}+z_{3}^{2}+z_{4}^{2}\right)} .
\end{aligned}
$$

where $r_{2,3,4}$ are the Kähler parameters controlling the sizes of the associated exceptional divisors in $M_{1}$.

Choosing $r_{2,3,4}>0$ we find a geometric phase. The real slice will have several identical components of which we choose one and call it $\Sigma$. Since the function $Q$ defined above is positive semidefinite, this component will shrink when $\epsilon \rightarrow 0$.

The analysis of the topology of this component $\Sigma$ of the real slice is included in the appendix. The conclusion is that in the regime of Kähler moduli considered above, $b_{1}(\Sigma)=5$. In particular, $\Sigma$ is a connected sum of 5 copies of $S^{1} \times S^{2}$. The mirror $\mathbb{P}^{1}$ has a one-dimensional moduli space, while by McLean's theorem $\Sigma$ would move in a 5-dimensional family. Thus we are guaranteed the presence of a disc-instanton generated superpotential which lifts four of the flat directions.

\section{Mirror symmetry and the superpotential}

We are interested in computing the superpotential for the A-type examples in $\S 3$, by finding a mirror map for the open string moduli. In this section we will make some progress in this direction. We will start in $\S 4.1$ by arguing that the features 
of the mirror B-type examples near the toric locus are captured by certain features of disc instantons in our A-type examples. In $\S 4.2$ we will find a large-complexstructure limit of the B-type examples for which the disc instantons of the mirror three-cycle will be large, and construct a mirror map for the chiral multiplet in this limit.

\subsection{The superpotential near the toric locus}

Recall that for the Ur-example and for example II, mirror symmetry requires the following story. The ambient Calabi-Yau $M$ (resp. $M_{2}$ ) has $g$ non-toric deformations with $g=3$ (resp. 1); these arise because the hypersurface intersects $g$ of the divisors of the (orbifolded) weighted projective space twice, to create two divisors in the hypersurface. At the "toric locus" these divisors have the same size. At this point the three-cycles we study must have a one-dimensional moduli space (namely a genus-g curve). As we leave this locus, we acquire a superpotential and are left with $2 g-2$ isolated three-cycles. We argue here that there will be different disc contibutions which cancel on the toric locus.

In our A-cycle examples, the defining equation for the threefold $M$ may be written as:

$$
0=\left(z_{1}^{4}-z_{2}^{4}\right)^{2}-2 \epsilon z_{1}^{4} z_{2}^{4}+Q
$$

where $Q$ is a function of all the variables $z_{k>2}$ other than $z_{1}$ and $z_{2}$. It follows that on the hypersurface:

$$
\left(z_{1}^{4}\right)_{ \pm}=z_{2}^{4}(1+\epsilon \pm \sqrt{A-Q})
$$

with $A=2 \epsilon+\epsilon^{2}$. Consider the map, $i: M \rightarrow M$, which fixes $z_{k>2}$ but flips the branches of $z_{1}^{4}$. We claim that this is an isometry of $M$ at the toric locus. Note that $i$ induces a map on the toric part of the cohomology: $i^{*}: H_{\text {toric }}^{2}(M) \rightarrow$ $H_{\text {toric }}^{2}(M)$. This happens to be the identity and so preserves the Kähler class on $M$. Furthermore, it preserves the complex structure, and so by Yau's theorem [93] it preserves the metric on $M$.

The non-toric Kähler deformations are odd under $i^{*}$. To see this, let us describe them in more detail following [89]. In our examples $M$ is a hypersurface in the quotient $\mathbb{P}_{1,1,2,2,2}^{4} / \Gamma$, where $\Gamma$ is the relevant Greene-Plesser (GP) (sub)group. If $\Gamma$ has elements which fix the locus $z_{3}=z_{4}=z_{5}=0$ (and not just varieties which contain it such as $z_{3}=z_{4}=0$ ), this locus must be blown up to desingularize $M$. The 
Kähler parameters controlling these blow-ups have a natural mirror description in toric geometry $[88,89]$. Within the lattice of exponents of monomials in the ambient space of $W$, the allowed monomials (preserved by the subgroup of the GP group complementary to $\Gamma$ ) lie on a polyhedron. The lattice points on the lines and faces of this polyhedron correspond to divisors in $M$ and monomial deformations of $W$. In particular the lattice points corresponding to $z_{3,4,5}^{4}$ in $W$ form the vertices of a triangular face of this polyhedron and control the monomial deformations of the Riemann surface $\mathcal{S}_{g} \subset W$. The $g$ interior points can be used to construct the holomorphic differentials of $\mathcal{S}_{g}$, so they are associated to the non-toric deformations which destroy the family of $\mathbb{P}^{1}$ s over $\mathcal{S}_{g}$.

In $M$ these $g$ interior points denote the Kähler parameters controlling the blowup of $z_{3}=z_{4}=z_{5}=0$. The exceptional divisors intersect the hypersurface twice in $M$, at the loci $\left(z_{1}^{4}\right)_{ \pm}=z_{2}^{4}(1+\epsilon \pm \sqrt{A})$. The non-toric moduli control the relative sizes of these divisors in $M$. Since the map $i$ defined above interchanges these two loci, and the non-toric deformations change their relative sizes, $i$ can no longer be an isometry away from the toric locus. Furthermore, at the toric locus $i$ will change the sign of the non-toric deformation.

The map $i$ lifts naturally to a map on holomorphic discs. Hence, these discs should come in pairs related by $i$. It is plausible that on the toric locus, the sign of the contribution in Eq. (2.13) is changed by $i$; then the disc instanton contributions of the pair will cancel in the superpotential. This could come about through the action of $i$ on fermion zero modes or on the pfaffian. When $i$ is not an isometry, the areas of the discs related by $i$ will differ, so their contributions to $W$ will no longer cancel.

Note that this cancellation cannot happen for every disc at the toric locus. In particular, in example I we find that $b_{1}(\Sigma)=5$ while the true moduli space is one dimensional. ${ }^{21}$ This suggests that the involution $i$ changes the sign of the contribution of discs with boundaries in only one class of $H_{1}(\Sigma)$. There is clearly much to understand here.

The set of examples we have considered fits into the more general framework discussed in [89]. The B-model CY in general contains a family of $A_{N}$ singularities

21 At special degenerate points in the complex structure moduli space of $W$, the dimension of the moduli space of the mirror $\mathbb{P}^{1}$ enlarges to two, but never to five. 
fibered over a genus $g$ curve. Upon resolving this family, one then finds $N$ families of $\mathbb{P}^{1}$ s. There are $g$ interior points on the relevant face of the toric diagram, and therefore $g$ non-toric complex structure deformations which destroy each family. Furthermore, the defining equation for the A-model CY will be of degree $N+1$ in one of the variables, $x$, which is single-valued under the GP orbifold (the analog of $x=z_{1}^{4}$ ), leading to $N+1$ branches of solutions. $g$ toric divisors each intersect these $N+1$ branches once. On the toric locus, the Galois group, $S_{N+1}$, of the defining equation will act via isometries on the CY by interchanging the branches of solutions for $x$, leading to cancellations between discs. Upon turning on the non-toric Kähler moduli, these isometries are broken allowing a nontrivial superpotential.

\subsection{The mirror map}

One of our long-term goals is to use mirror symmetry to find the explicit form of the instanton sums for A-type branes. Of course this sum is automatically computed in the B-model, but we require a mirror map for the open string fields in order that this be of any use. In the context of our models, this means the following. The B-brane moduli space is parametrized by the complex coordinate $z$ on a genus $g$ surface, while the A-brane moduli space is parametrized via (2.9) by the disc area $A$ and Wilson line $a$. Thus, defining

$$
q=e^{-\phi}
$$

we would like to find a map $z(q)$.

As in the closed string case, this will be easiest around "large radius" or "large complex structure" points, in particular when $\operatorname{Re}(\phi)$ is large so that the instanton action is small and classical geometry is a reasonable guide. We therefore search for a map in a region of large radius of our IIA models, and in the mirror large complex structure limit of our IIB models. In this limit we can identify the mirror of the large-disc limit of the A-cycle moduli space with a particular point on the B-cycle moduli space. Finally, since the superpotential is explicitly computable in the Bmodel side, we can use our previous intuition about the A-type superpotentials to guess at an explicit mirror map in this limit.

We will work exclusively with the Ur-Example in this section.

The large-complex-structure limit of $W$ 
On the manifold $M$, we are interested in the large radius limit. In particular, the sizes of discs ending on the real slice are determined by sizes of rational curves (as shown in $\S 2.3$ ), so we demand that the exceptional divisors which intersect the three-cycle are large. The mirror locus in the complex structure moduli space of $W$ is specified by the defining equation

$$
p=\alpha z_{3}^{2} z_{4} z_{5}+\beta z_{3} z_{4}^{2} z_{5}+\gamma z_{3} z_{4} z_{5}^{2}=0
$$

with $\alpha, \beta, \gamma$ large and at fixed ratios (recall that these monomials correspond to the toric divisors in $\left.M_{1}\right)$. (4.4) can be rewritten as

$$
z_{3} z_{4} z_{5}\left(\alpha z_{3}+\beta z_{4}+\gamma z_{5}\right)=0
$$

This degeneration is similar to the large complex structure limit discussed in [94]. It is described by four $\mathbb{P}^{3}$ s at $z_{3}=0, z_{4}=0, z_{5}=0$ and $\alpha z_{3}+\beta z_{4}+\gamma z_{5}=0$ which intersect as shown in the figure.

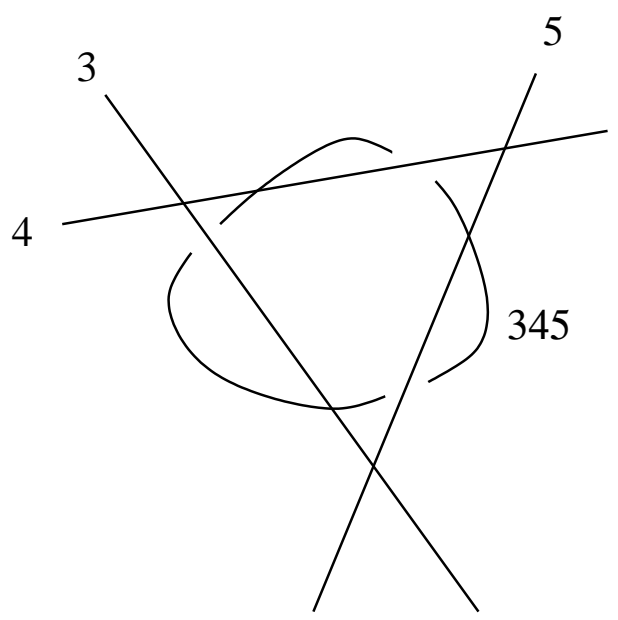

Fig. 2: $M$ in the large complex structure limit. The lines represent $\mathbb{P}^{3} \mathrm{~s}$ labeled by which coordinate vanishes on them. ' 345 ' denotes the one where $\alpha z_{3}+\beta z_{4}+\gamma z_{5}=0$. This picture also accurately portrays the degeneration of the curve $\mathcal{S}_{3}$ where $z_{1}=z_{2}=0$, in which case the lines represent $\mathbb{P}^{1} \mathrm{~s}$.

Each $\mathbb{P}^{3}$ is identical to the others and joins them in a symmetrical way. As in [94] where the example of the quintic at large complex structure is discussed, in this large complex structure limit of $W$ it is easy to write down a flat Kähler metric. Let us examine the metric on $z_{3}=0$ near the locus $z_{4}=0$. We may use 
the $\mathbb{C}^{*}$ action on the $W \mathbb{P}^{4}$ to set $z_{5}=1$. The standard residue formula yields the following expression for the holomorphic $(3,0)$ form $\Omega$ in this degenerate limit:

$$
\Omega=\int_{p=0} \frac{d z_{1} \ldots d z_{5}}{p}=\frac{d z_{1} d z_{2} d z_{4}}{z_{4}\left(\beta z_{4}+\gamma\right)}
$$

Near $z_{4}=0$ the Ricci-flat metric is clearly:

$$
d z_{1}^{2}+d z_{2}^{2}+\frac{1}{\gamma^{2}} d\left(\ln z_{4}\right)^{2}
$$

Elsewhere on the slice it is:

$$
d z_{1}^{2}+d z_{2}^{2}+d \zeta^{2}
$$

where

$$
\zeta=\frac{1}{\gamma} \ln \frac{\beta z_{4}+\gamma}{z_{4}}
$$

$\mathcal{S}_{g}$ in this limit lives at $z_{1}=z_{2}=0$. It is a chain of four $\mathbb{P}^{1} \mathrm{~s}$, one in each $\mathbb{P}^{3}$ and joined as in fig. 2. The genus-3 structure is clear from this figure. The metric is simply induced from Eq. (4.9). In particular it is clear that the different components are joined along infinite cylinders, parameterized by $\ln z_{i}$ for $z_{i} \rightarrow 0$. This then is the asymptotic moduli space for a D5-brane wrapped on an element of the family of $\mathbb{P}^{1}$ s at $z_{3}=0$ near $z_{4}=0$.

\section{The large-radius limit of $M$}

In the mirror $M$ there are three toric divisors which are taken to be large: near the toric locus, this means that all relevant divisors are large since the toric modulus controls the sum of the sizes of the exceptional divisors.

The moduli of the A-type branes are the areas of discs; according to our discussion at the end of $\S 2.3$ these discs live in pairs forming $\mathbb{P}^{1} \mathrm{~s}$ in the Poincaré dual class of these toric divisors. When the brane wraps a real slice it bisects these $\mathbb{P}^{1} \mathrm{~s}$. Let us parameterize the $\mathbb{P}^{1}$ by an altitudinal angle $\theta$ and an azimuthal angle $\rho$. The equator is $\theta=0$ and the real slice intersects this equator. Let $2 \pi R$ be the circumference of the equator. $R$ will be a function of $\alpha, \beta, \gamma$ via the mirror map for closed strings. As the three-cycle moves through its moduli space, it will sweep out the $\mathbb{P}^{1}$ by intersecting it at fixed $\theta$. We choose the open-string modulus so that 
$\phi$ is the area of the smaller disc. For $\theta$ close to zero, a natural metric on the disc coordinate is:

$$
d s^{2}=d A^{2}+d a^{2}=2 \pi R^{4} \cos \theta(d \theta)^{2}+\frac{1}{(2 \pi R)^{2} \cos ^{2} \theta}(d \alpha)^{2},
$$

where $\alpha \in[0,2 \pi]$. Near $\theta=0$ we can set $\cos \theta=1$ and this is clearly a cylinder, with the periodic direction given by the Wilson line.

Asymptotic identification of the coordinates

Let us take $\beta, \gamma \in \mathbb{R}$. Up to overall normalizations of the fields we roughly identify

$$
\ln z_{4}=\phi
$$

due to the periodicity of the imaginary parts of each side of this equation. This use of the periodicity is similar to the use of monodromy properties in identifying the closed string mirror map. Note that the correct normalization of the fields is extracted from the so far unknown Kähler metric. Thus our map is only good up to some overall constant. 


\section{Computing the superpotential}

As reviewed in $\S 2$, the deformation along the non-toric locus can be specified by the choice of a holomorphic differential. Let $\tilde{p}$ be the defining polynomial for $\mathcal{S}_{g} \subset W$ at $z_{1}=z_{2}=0$. The general holomorphic differential can be writtten as:

$$
\omega=\int_{\tilde{p}=0} \frac{z_{3} d z_{4} d z_{5}+z_{4} d z_{3} d z_{5}+z_{5} d z_{3} d z_{4}}{\tilde{p}}\left(a z_{3}+b z_{4}+c z_{5}\right) .
$$

On the locus $z_{3}=0$, this becomes:

$$
\omega=\frac{d z_{5}}{z_{5}\left(\beta z_{4}+\gamma z_{5}\right)}\left(b z_{4}+c z_{5}\right)+\frac{d z_{4}}{z_{4}\left(\beta z_{4}+\gamma z_{5}\right)}\left(b z_{4}+c z_{5}\right) .
$$

We can concentrate on the region near $z_{4}=0$ by using the $\mathbb{C}^{*}$ action of $W$ to fix $z_{5}=1$. Then

$$
\omega=\frac{d z_{4}}{z_{4}\left(\beta z_{4}+\gamma\right)}\left(b z_{4}+c\right) .
$$

In the flat coordinates $x$ we write $\omega=f(x) d x$. As shown in [69], $W^{\prime}(\Phi)=f(\Phi)$ for the associated chiral multiplet. This superpotential clearly has a single vacuum at $z_{4}=-c / b$. It is easy to see that there is a single such vacuum in each of the $\mathbb{P}^{1}$ components of $\mathcal{S}_{3}$ in this limit, for a total of $2 g-2=4$ isolated vacua.

We wish to make contact with the large-disc limit of the toric locus of the Acycle moduli space. Therefore we push the vacuum to infinite distance by sending $c \rightarrow 0$. In this limit,

$$
W_{\zeta}(\zeta)=b z(\zeta)=\frac{\gamma}{e^{\gamma \zeta}-\beta}
$$

for the superpotential of the B-brane. If the A-cycle superpotential were dominated by a single pair of discs, the corresponding superpotential would be that in Eq. (2.15). Certainly as $z_{4} \rightarrow 0$ and $\phi \rightarrow \infty,(4.15)$ and (2.15) are equal to lowest order in $z_{4}$ and $e^{-\phi}$, given (4.11).

Our candidate superpotentials are equal only to lowest order as we only have an asymptotic mirror map at present. There are several complications in constructing an exact mirror map. First, given our experience with Example I, we expect that the three-cycle has a sizable number of classical moduli. All but one gain masses from instanton corrections, but the remaining moduli space may be a nontrivial submanifold of the classical moduli space. So our formulae for the superpotential as a function of this modulus are undoubtedly rather schematic. 
Secondly, we have assumed that the only contributions are a disc $D \in H_{2}(M, \Sigma)$ plus its three images arising from the anti-holomorphic involution and the map $i$ discussed in $\S 4.1$. Of course there may be higher-degree contributions $n D$ which are not multiple covers, and there may be contributions from discs $D^{\prime}$ for which $\left[D-D^{\prime}\right]$ lies in a nontrivial class in $H_{2}(M)$. We leave these issues for future work.

Finally, we have not yet found a global topology of the moduli space of Abranes which matches the topology of the moduli space of the B-branes, even in this degenerate limit.

\section{Discussion}

In this chapter, we have presented explicit examples of three-cycles with nontrivial topology which are mirror to two-cycles which have either obstructed holomorphic deformations or no deformations. After the results of [69], this clearly shows that there is a disc instanton generated superpotential for the moduli of such cycles. We have certainly not given a complete formulation of the mirror map in these examples, but we have made a first step by presenting an analog of the monomial-divisor mirror map for closed string moduli [95].

Although we lack the full power of $\mathcal{N}=2$ special geometry that exists for closed string mirror symmetry, the structure of the $\mathcal{N}=1$ theory we are studying gives us some information. In particular, the superpotential $W$ must be holomorphic in the appropriate variables. Therefore, in limits where one has an open string modulus $\phi$ which is periodic with period $2 \pi i$, the superpotential must be a holomorphic function of $e^{\phi}$. This together with some detailed knowledge of the behavior of $W$ at singularities should be enough to determine the function entirely.

We can also draw some general lessons from this work and the results of [69]. As with closed strings, in making mathematical statements using mirror symmetry one must take the instanton corrections into account. For instance, there is a general conjecture that fundamentally, mirror symmetry is a relation between the Lagrangian submanifolds of a threefold and the semistable coherent sheaves on its mirror [96]. The fact that stringy nonperturbative effects prevent generic special Lagrangian three-cycles from being supersymmetric indicates that this comparison will be complicated. 
It would be interesting to study these issues in the presence of orientifolds (required for tadpole cancellation), as a step towards genuine model-building. ${ }^{22}$ Many of the results of this chapter and $[67,69]$ rest on the fact that from the $\sigma$ model point of view, the superpotential is essentially a topological quantity and can be computed in an appropriately twisted theory. Since $\mathcal{N}=2$ worldsheet supersymmetry is a consequence of $\mathcal{N}=1, d=4$ spacetime supersymmetry [97], the twisted theories will still make sense in the presence of orientifolds.

Another subject worth exploring is the behavior of the topology of a given special Lagrangian cycle $\Sigma$ as the closed string parameters vary. It is clear from some of our examples that the topology of $\Sigma$ can change as one varies Kähler parameters of the ambient Calabi-Yau space. For instance, in the example we discuss in Appendix A, different choices of the blow-up parameters $r_{2,3,4}$ yield three-cycles of different topology in the same homology class.

\section{Acknowledgements}

We would like to thank M.R. Douglas, Y. Eliashberg, A. Klemm, D. Morrison, J.R. Myers, M.R. Plesser, S. Sethi, C. Vafa, E. Zaslow, and Y. Zunger for helpful communications. We thank P. Kaste for pointing out an error in an earlier version of this work. S. Kachru was supported in part by an A.P. Sloan Foundation Fellowship, and by the DOE under contract DE-AC03-76SF00515. S. Katz was supported in part by NSA grant number MDA904-98-1-0009, and would like to thank the Stanford High Energy Theory Group for their hospitality during the course of this project. A. Lawrence was supported in part by the DOE under contract DE-AC0376SF00515 and in part by a DOE OJI grant awarded to E. Silverstein; he would like to thank the University of Chicago High Energy Theory Group, the Rutgers High Energy Theory Group, and especially the Oklahoma State University Department of Mathematics for their hospitality during the course of this project. J. McGreevy was supported in part by the Department of Defense NDSEG Fellowship program. This project received additional support from the American Institute of Mathematics.

22 We would like to thank S. Sethi for a discussion of these points. 


\section{Appendix A. Some Details Concerning Example I}

In this appendix we determine the topology of $\Sigma$, the component of the real slice of the mirror of $\mathbb{P}_{1,1,2,2,2}[8]$ on which all of the $w_{i}$ are positive. We will choose a regime in moduli space where

$$
\epsilon>>r_{i}, \quad r_{1}>>r_{i}
$$

for all $i \geq 2$.

As in the simpler examples, we solve the defining equation for $x=z_{1}^{4}$ by

$$
x=1+\epsilon \pm \sqrt{A-Q} .
$$

We have set $z_{2}=1$ again. We will see that the other variables charged under $\mathbb{C}_{1}^{*}$ are bounded on $\Sigma$ and so $\Sigma$ is entirely contained in this coordinate patch. The locus $B \equiv\{Q=A\}$ where the two branches of $x$ are joined is a big (not quite round) ball in the $\mathbb{R}^{3}$ coordinatized by $z_{3,4,5}$. The branches of $x$ are then two copies of this ball glued along the boundary. The loci where the branches of $z_{6,7,8}$ are joined are three tubes surrounding the coordinate axes and ending on $B$. The region where all variables are real is the part of the inside of $B$ which is outside the union of these tubes. Suppose we are in a regime of moduli where $r_{3}+r_{4}<r_{2}$. Then the tubes surrounding the $z_{3}$ and $z_{4}$ axes will both intersect the one surrounding the $z_{5}$ axis, but not each other, like this:

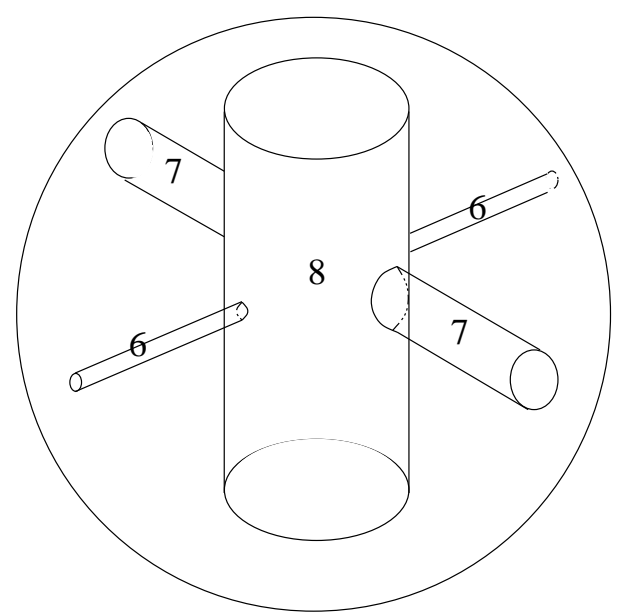

Fig. 3: The real slice is the ball with the tubes removed. The tubes are labeled according to which branches are glued along them.

Now divide by the orbifold group which maps the real slice to itself. It acts by flipping signs in pairs:

$$
\left(z_{3}, z_{4}, z_{5}\right) \sim\left(z_{3},-z_{4},-z_{5}\right) \sim\left(-z_{3}, z_{4},-z_{5}\right) \sim\left(-z_{3},-z_{4}, z_{5}\right) .
$$




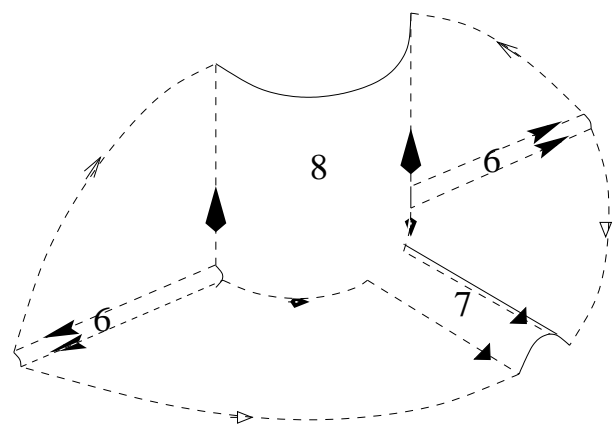

Fig. 4: A fundamental domain for the orbifold action on the real slice (glue along the dotted lines with matching arrows).

After performing this identification, the plumbing fixture surrounding the origin depicted in fig. 3 becomes a half-cigar (where $z_{8}=0$ ) ending on $B$ with two smaller tubes coming off of it (where $z_{6}=0$ and $z_{7}=0$ respectively) and ending on $B$ as well.

Next, glue the two $x$-branches along $B$. This produces an $S^{3}$ with the following set removed: The locus where $z_{8}$ becomes imaginary is now a full cigar, and the $z_{6}=0$ and $z_{7}=0$ loci are two handles coming off of this cigar.

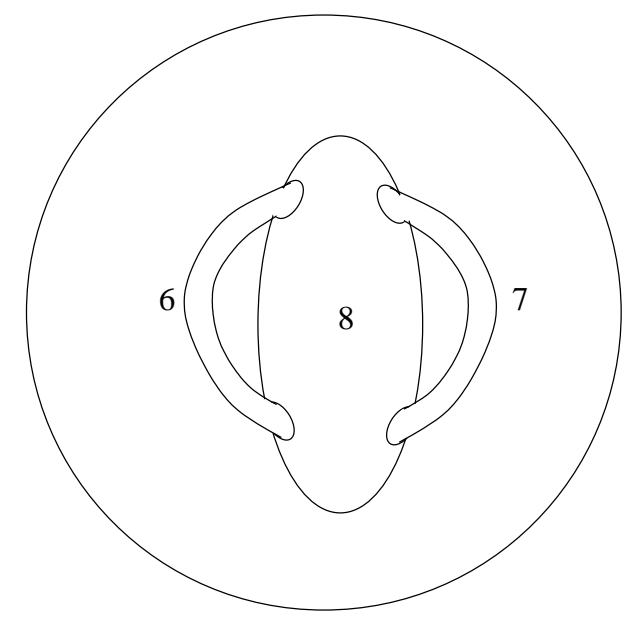

Fig. 5: The real slice is the ball with the blob in the middle excised.

An $S^{3}$ with the $z_{8}$-cigar removed is again a three-ball; the two handles coming off of the cigar become tunnels through this three-ball.

We take four copies of this creature to represent the two branches each of $z_{6}$ and $z_{7}$. They are glued in pairs along the tunnels. To see what this is we must use the fact that gluing handlebodies along a tunnel is the same as gluing along a tube that contains a handle. 


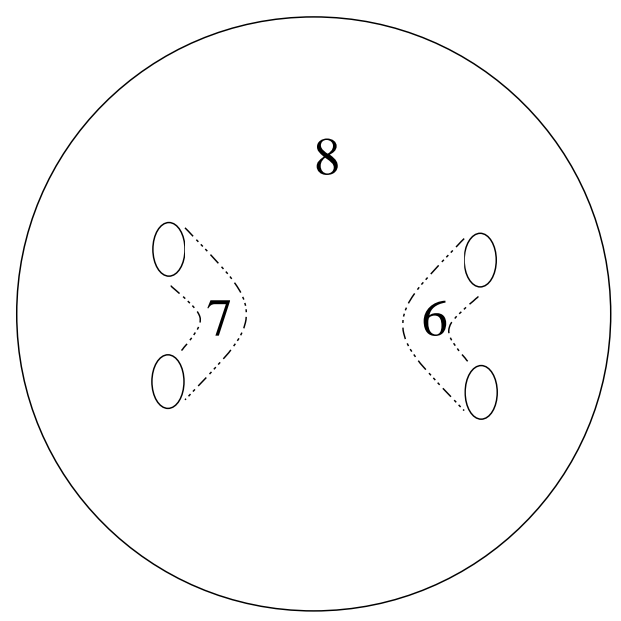

Fig. 6: The previous picture turned inside-out. The real slice is now the inside of the ball minus the two tunnels. The boundary of the ball is where $z_{8}=0$.

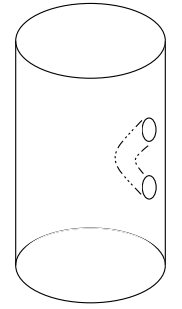

a

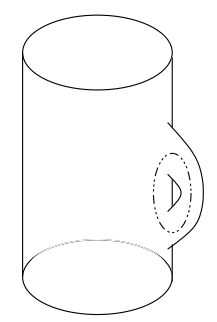

b

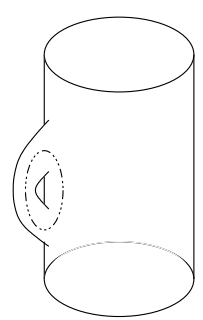

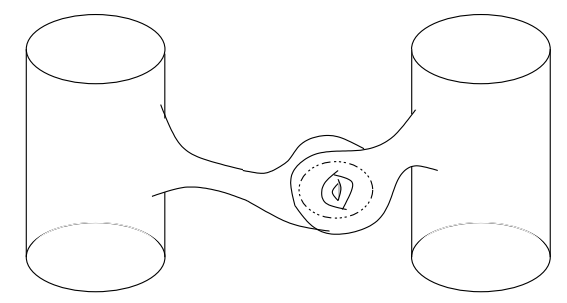

$\mathrm{C}$

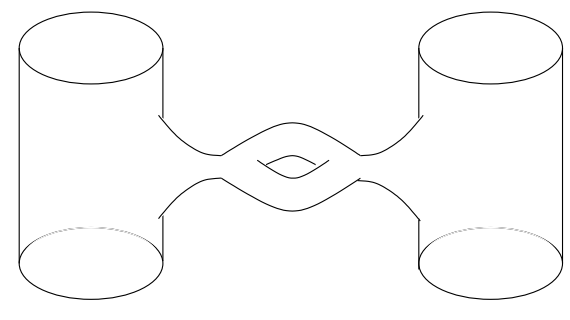

d

Fig. 7: Gluing handlebodies (in particular, solid cylinders) along a tunnel is the same as connecting them via a tube with a handle in it.

After we do this gluing, we find a solid genus 5 surface for each branch of $z_{8}$. The boundary of this surface is where $z_{8}=0$.

Since the two different branches for $z_{8}$ meet at $z_{8}=0$, we now glue two copies of the genus 5 surface together along their boundaries. In general, two solid genus $g$ surfaces glued in this manner describe a Heegaard splitting of a connected sum of $g$ copies of $S^{2} \times S^{1}$. Hence, this three-cycle has $b_{1}(\Sigma)=5$. 


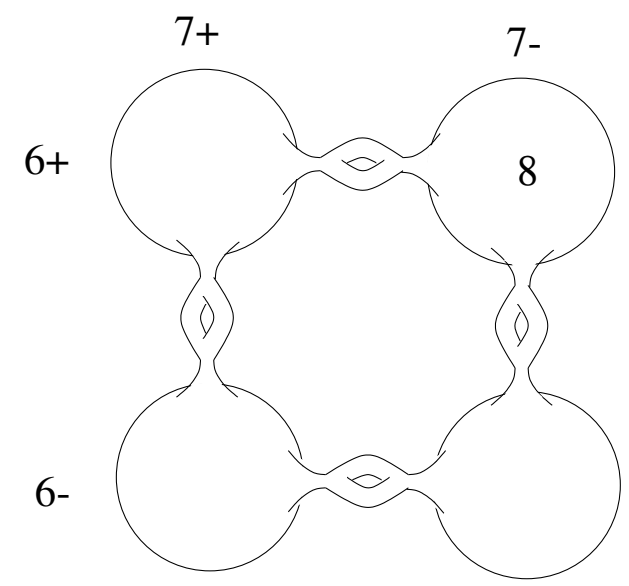

Fig. 8: Our special Lagrangian three-cycle is obtained by gluing two of these along their boundaries via the trivial identification. The numbers along the top and left indicate which branch each ball represents.

\section{Chapter 5: Invasion of the Giant Gravitons from Anti de Sitter Sp}

It has been known for some time that the AdS/CFT correspondence predicts a limit on the number of single particle states propagating on the compact spherical component of the $A d S \times S$ geometry. The limit is called the stringy exclusion principle. The physical origin of this effect has been obscure but it is usually thought of as a feature of very small distance physics. In this chapter we will show that the stringy exclusion principle is due to a surprising large distance phenomenon. The massless single particle states become progressively less and less point-like as their angular momentum increases. In fact they blow up into spherical branes of increasing size. The exclusion principle is simply understood as the condition that the particle should not be bigger than the sphere that contains it.

The material in Chapter 4 appeared in "Invasion of the Giant Gravitons from Antide Sitter Space" with Leonard Susskind and Nicolaos Toumbas [98] and is reprinted with permission of JHEP. 


\section{Introduction}

In conventional (20th century) physics, high energy or high momentum came to be associated with small distances. The physics of the 21 st century is likely to be dominated by a very different perspective. According to the Infrared/Ultraviolet connection [99] which underlies much of our new understanding of string theory and its connection to gravity, physics of increasing energy or momentum is governed by increasingly large distances. Examples include the growth of particle size with momentum [100], [101], the origin of the long-range gravitational force in Matrix Theory from high energy quantum corrections [102] and the IR/UV connection in AdS spaces. Another important manifestation is the spacetime uncertainty principle of string theory [103], [104], [105]

$$
\Delta x \Delta t \sim \alpha^{\prime} .
$$

Similar uncertainty principles occur in non-commutative geometry where the coordinates of space do not commute. An important consequence of the noncommutativity is the fact that the particles described by non-commutative field theories have a spatial extension which is proportional to their momentum [106], [107]. This in turn leads to unfamiliar violations of the conventional decoupling of IR and UV degrees of freedom in these theories [108], [109], [110]. In this chapter we will describe another example of IR/UV non-decoupling that occurs in AdS/CFT theories. The relevant space-times have the form $A d S_{n} \times S^{m}$. We are interested in the motion of the graviton and other massless bulk particles on the $S^{m}$. The motion is characterized by an angular momentum $L$ or more exactly a representation of the rotation group $O(m+1)$. In 20th century physics such particles are regarded as point or almost point particles regardless of $L$. In fact we will see that as $L$ increases the particles blow up in size very much like the quanta of non-commutative field theories. When the size reaches the radius of the $S^{m}$, the growth can no longer continue and the tower of Kaluza-Klein states terminates. This is the origin of the stringy exclusion principle [111], [112], [113].

In section 2 we will review the theory of electric dipoles moving in a magnetic field. This system is the basic object of non-commutative field theory. When the theory is defined on a 2-sphere there is a bound on the angular momentum when the ends of the dipole separate to the antipodes of the sphere. In sections 3,4 and 5 we consider the cases of $A d S_{7} \times S^{4}, A d S_{4} \times S^{7}$ and $A d S_{5} \times S^{5}$. In each case we

find that the spectrum of angular momentum is bounded and that the bound agrees with expectations from the stringy exclusion principle. 


\section{Dipoles in Magnetic Fields}

In this section we briefly review the dipole analogy for non-commutative field theory [106], [107]. We begin with a pair of unit charges of opposite sign moving on a plane with a constant magnetic field $B$. The Lagrangian is

$$
\mathcal{L}=\frac{m}{2}\left(\dot{x}_{1}^{2}+\dot{x}_{2}^{2}\right)+\frac{B}{2} \epsilon_{i j}\left(\dot{x}_{1}^{i} x_{1}^{j}-\dot{x}_{2}^{i} x_{2}^{j}\right)-\frac{K}{2}\left(x_{1}-x_{2}\right)^{2} .
$$

Let us suppose that the mass is so small so that the first term in Eq.(2.1) can be ignored. Let us also introduce center of mass and relative coordinates

$$
\begin{aligned}
X & =\left(x_{1}+x_{2}\right) / 2 \\
\Delta & =\left(x_{1}-x_{2}\right) / 2 .
\end{aligned}
$$

The Lagrangian becomes

$$
\mathcal{L}=B \epsilon_{i j} \dot{X}^{i} \Delta^{j}-2 K \Delta^{2}
$$

From Eq.(2.3) we first of all see that $X$ and $\Delta$ are non-commuting variables satisfying

$$
\left[X^{i}, \Delta^{j}\right]=i \frac{\epsilon^{i j}}{B}
$$

Furthermore the center of mass momentum conjugate to $X$ is

$$
P_{i}=B \epsilon_{i j} \Delta^{j}
$$

Thus when the dipole is moving with momentum $P$ in some direction it is stretched to a size

$$
|\Delta|=|P| / B
$$

in the perpendicular direction. This is the basis for the peculiar non-local effects in non-commutative field theory.

Now suppose the dipole is moving on the surface of a sphere of radius $R$. Assume also that the sphere has a magnetic flux $N$. In other words there is a magnetic monopole of strength

$$
2 \pi N=\Omega_{2} B R^{2}
$$


at the center of the sphere. We can get a rough idea of what happens by just saying that when the momentum of the dipole is about $2 B R$ the dipole will be as big as the sphere. At this point the angular momentum is the maximum value

$$
L=P R \sim B R^{2}
$$

This is of order the total magnetic flux $N$.

We will now do a more precise analysis and see that the maximum angular momentum is exactly $N$. Parameterize the sphere by two angles $\theta, \phi$. The angle $\phi$ measures angular distance from the equator. It is $\pm \pi / 2$ at the poles. The azimuthal angle $\theta$ goes from 0 to $2 \pi$. We work in a gauge in which the $\theta$ component of the vector potential is non-zero. It is given by

$$
A_{\theta}=N \frac{1-\sin \phi}{2 R \cos \phi}
$$

For a unit charged point particle moving on the sphere the term coupling the velocity to the vector potential is

$$
\mathcal{L}_{A}=A_{\theta} R \cos \phi \dot{\theta}=N R \frac{1-\sin \phi}{2 R} \dot{\theta}
$$

Now consider a dipole with its center of mass moving on the equator. The positive charge is at position $(\theta, \phi)$ and the negative charge is at $(\theta,-\phi)$. For the motion we consider $\phi$ is time independent. Eq.(2.10) becomes

$$
\mathcal{L}_{A}=N\left(\frac{1-\sin \phi}{2}\right) \dot{\theta}-N\left(\frac{1+\sin \phi}{2}\right) \dot{\theta}
$$

or

$$
\mathcal{L}_{A}=-N \sin \phi \dot{\theta}
$$

Again we want to consider a slow-moving dipole whose mass is so small that its kinetic term may be ignored compared to the coupling to the magnetic field, i.e. $m R<<N$. Let us also add a spring coupling

$$
\mathcal{L}_{S}=-\frac{k}{2} R^{2} \sin ^{2} \phi
$$

for simplicity, we have used the chordal distance in this potential. The total Lagrangian is

$$
\mathcal{L}=-\frac{k}{2} R^{2} \sin ^{2} \phi-N \sin \phi \dot{\theta}
$$


and the angular momentum is

$$
L=-N \sin \phi
$$

The angular momentum will reach its maximum when $\phi=\pi / 2$ at which point

$$
\left|L_{\max }\right|=N
$$

The fact that the angular momentum of a single field quantum in non-commutative field theory is bounded by $N$ is well known in the context of non-commutative field theory on a sphere [114]. Here we see that it is a large distance effect.

\section{3. $A d S \times S$}

We now study BPS particles moving on the sphere of maximally supersymmetric $A d S$ vacua of string and M theory. For simplicity, we present all details of the argument in the case of the M5-brane geometry. Results for the other cases are given in the latter subsections. Note that in all of these cases, the energy of our objects is well below the energy of a stable AdS black hole.

\section{1. $A d S_{7} \times S^{4}$}

We are interested in the motion of a BPS particle on the 4-sphere of $A d S_{7} \times S^{4}$. We will assume that the radius of curvature $R$ is much larger than the 11 dimensional Planck length $l_{p}$. The analogy with the previous example is very close. The role of the magnetic field is played by the 4-form field strength on the sphere. We call the flux density B. Quantization of flux requires

$$
\Omega_{4} B R^{4}=2 \pi N
$$

From the supergravity equations of motion it can be seen [115] that $R$ is given by

$$
R=l_{p}(\pi N)^{\frac{1}{3}}
$$

The assumption of large $R$ means $N>>1$.

We want to know what happens to a graviton or any other massless 11 dimensional particle when it moves on the 4-sphere in the presence of the 4-form field strength. As long as the angular momentum is small $(L<<N)$ the graviton is 
expected to be much smaller than $R$, and we can make the approximation that space is locally flat. Furthermore from Eq.(3.1) we see that the field strength $B$ is small

$$
B \sim N^{-\frac{1}{3}} l_{p}^{-4}
$$

Since the space is almost flat we can locally introduce flat space coordinates $x^{0}, \ldots, x^{10}$. Let us take the $B$ field to lie in the $(7,8,9,10)$ directions. The graviton is moving along the $x^{10}$ direction. Its momentum is $P_{10}=L / R$. This setup is very close to one that was studied by Myers using Matrix Theory [116].

In matrix theory the 11 dimensional graviton is viewed as a threshold bound state of $n=P_{10} R_{10}$ D0-branes. Myers shows that in a background 4 -form fieldstrength the D0-brane configuration is described as a spherical membrane with a radius $r$ that grows with $P_{10}$ according to

$$
r \sim B P_{10} l_{p}^{6}
$$

Let us assume that this formula is approximately valid until $r$ becomes of order $R$. In that case when the graviton size becomes $\sim R$ it will have momentum

$$
P_{10}(\max ) \sim R / B l_{p}^{6}
$$

and angular momentum

$$
L_{\max } \sim R P_{10}(\max ) \sim R^{2} / B l_{p}^{6} \sim N
$$

Thus as in the previous section we find that the maximum single particle angular momentum is $N$.

We will now give a more precise calculation which parallels that of section 1. We are interested in the dynamics of a relativistic spherical membrane moving in $S^{4}$. The membrane has zero net charge but it couples to the background field strength. It behaves like the dipole of section 1.

Let us parametrize $S^{4}$ using cartesian coordinates $X_{1}, \ldots ., X_{5}$ so that

$$
\begin{array}{r}
X_{1}=R \cos \theta_{1} \\
X_{2}=R \sin \theta_{1} \cos \theta_{2} \\
X_{3}=R \sin \theta_{1} \sin \theta_{2} \cos \theta_{3} \\
X_{4}=R \sin \theta_{1} \sin \theta_{2} \sin \theta_{3} \cos \theta_{4} \\
X_{5}=R \sin \theta_{1} \sin \theta_{2} \sin \theta_{3} \sin \theta_{4} .
\end{array}
$$


The angles $\theta_{1}, \ldots, \theta_{3}$ go from 0 to $\pi$. The angle $\theta_{4}$ is the azimuthal angle and goes from 0 to $2 \pi$. Then

$$
X_{1}^{2}+X_{2}^{2}+X_{3}^{2}+X_{4}^{2}+X_{5}^{2}=R^{2} .
$$

Next we embed a spherical membrane in $S^{4}$. We choose to parametrize the surface of the membrane by $\theta_{3}, \theta_{4}$. The brane is allowed to move in the $X_{1}, X_{2}$ plane. Its size depends on its location in the $X_{1}, X_{2}$ plane according to

$$
r=R \sin \theta_{1} \sin \theta_{2} .
$$

We see that when the size is at its maximum value, $r=R$, the membrane is at the origin $X_{1}=X_{2}=0$ like the two charges at the ends of the dipole in section 1. Since

$$
X_{1}^{2}+X_{2}^{2}=R^{2}-r^{2}
$$

the membrane can move around a circle in the plane and have constant size. We also set

$$
\begin{aligned}
& X_{1}=\sqrt{R^{2}-r^{2}} \cos \phi \\
& X_{2}=\sqrt{R^{2}-r^{2}} \sin \phi .
\end{aligned}
$$

In terms of the coordinates $r, \phi, \theta_{3}, \theta_{4}$, the metric on the 4 -sphere becomes

$$
d s^{2}=\frac{R^{2}}{\left(R^{2}-r^{2}\right)} d r^{2}+\left(R^{2}-r^{2}\right) d \phi^{2}+r^{2} d \Omega_{2}^{2},
$$

where $d \Omega_{2}^{2}$ is the metric of a unit 2-sphere parametrized by $\theta_{3}$ and $\theta_{4}$. From the metric we see that the volume element is just

$$
R r^{2} d r d \phi d \Omega_{2}
$$

The kinetic energy of the membrane is given by the Dirac-Born-Infeld Lagrangian. We are mostly interested in the case when the size of the sphere $r$ is constant and close to its maximum value $R$. In this case the membrane moves around a circle in the $X_{1}, X_{2}$ plane of radius $\left(R^{2}-r^{2}\right)^{1 / 2}$ with angular velocity $\dot{\phi}$. Dropping time derivatives of $r$, we have

$$
\mathcal{L}_{K}=-T \Omega_{2} r^{2} \sqrt{1-\left(R^{2}-r^{2}\right) \dot{\phi}^{2}}
$$


Here, $T$ is the membrane tension which is given by

$$
T=\frac{1}{4 \pi^{2} l_{p}^{3}}
$$

in 11-dimensional Planck units.

Next we add the Chern-Simons coupling involving the background field. The contribution of the four-form field strength to the action of the brane per orbit around the $S^{4}$ is

$$
S_{B}=\int_{w v} C=\int_{\Sigma} F .
$$

The first integral is over the world-volume of the brane. $F=d C$ is the fourform flux, and $\Sigma$ is a four-manifold in the $S^{4}$ whose boundary is the 3-dimensional surface swept out by the brane during one orbit. Since the background flux is just $F=B d$ vol, where $B$ is the constant flux density and $d$ vol is the volume form on $S^{4}$, we have

$$
S_{B}=B \operatorname{vol}(\Sigma) .
$$

Therefore the Chern-Simons term in the Lagrangian is

$$
\mathcal{L}_{B}=\frac{S_{B}}{T}=B \operatorname{vol}(\Sigma) \frac{\dot{\phi}}{2 \pi}
$$

where $\dot{\phi}$ is the (constant) angular velocity of the brane. Parametrizing the motion as above, the volume of $\Sigma$ is

$$
\operatorname{vol}(\Sigma)=R \Omega_{2} \int_{0}^{2 \pi} d \phi \int_{0}^{r} r^{\prime 2} d r^{\prime}=\frac{8 \pi^{2}}{3} R r^{3} .
$$

So the Chern-Simons term is

$$
\mathcal{L}_{B}=\frac{\dot{\phi}}{2 \pi} B \Omega_{4} R r^{3}=\dot{\phi} N \frac{r^{3}}{R^{3}}
$$

where we used the flux quantization condition, Eq.(3.1).

Therefore, the full bosonic Lagrangian is

$$
\mathcal{L}=-m \sqrt{1-\dot{\phi}^{2}\left(R^{2}-r^{2}\right)}+N \frac{r^{3}}{R^{3}} \dot{\phi}
$$

with $m=\Omega_{2} T r^{2}$. Using Eq.(3.2) and Eq.(3.15), we also see that

$$
\frac{N}{R^{3}}=T \Omega_{2} .
$$


From the Lagrangian we find that the angular momentum is given by

$$
L=\frac{m \dot{\phi}\left(R^{2}-r^{2}\right)}{\sqrt{1-\dot{\phi}^{2}\left(R^{2}-r^{2}\right)}}+N \frac{r^{3}}{R^{3}}
$$

The maximum size a membrane can have is $R$. Also, the velocity of its center of mass, $\dot{\phi} R$, cannot exceed the speed of light. This implies that the angular momentum has a maximum value given by $N^{23}$ :

$$
L_{\max }=N
$$

When the membrane has maximal size $R$, the angular momentum is the maximum value $N$. We see that the Kaluza-Klein graviton has a maximum angular momentum in agreement with the stringy exclusion principle. For the energy, we find

$$
E=\dot{\phi} L-\mathcal{L}=\sqrt{\left(\frac{N r^{2}}{R^{3}}\right)^{2}+\frac{\left(L-N r^{3} / R^{3}\right)^{2}}{R^{2}-r^{2}}} .
$$

Varying the energy with respect to $r$ at fixed $L$, we find

$$
\frac{d E}{d r}=\frac{r}{E\left(R^{2}-r^{2}\right)^{2}}\left(L-N \frac{r}{R}\right)\left(L-2 N \frac{r}{R}+N \frac{r^{3}}{R^{3}}\right) .
$$

We see that for $L<N$ there exists a stable minimum at

$$
r=\frac{L}{N} R
$$

Therefore, the membrane grows as we increase the angular momentum. This is in agreement with Eq.(3.4) ${ }^{24}$. When $r=R$ and $L=N$, a more careful analysis for the stability of the solution is needed and we do so at the end of this section. The value of the energy at the minimum is

$$
E=\frac{L}{R}
$$

23 There is an exception to this statement at the pathological value $r=0$ which we discuss at the end of this section.

24 The cubic factor in $d E / d r$ has a positive root at $0<r<(L / N) R$ at which the energy has a local maximum and another root at $r>R$. 
which is the energy of a Kaluza-Klein graviton with angular momentum $L$. From Eq.(3.23), we also find that the velocity of the center of mass equals the speed of light, $\dot{\phi} R=1$.

We now show that there is a stable solution at $r=R$ and $L=N$. Setting $\tilde{r}=R-r$ and expanding the Lagrangian up to quadratic powers in $\tilde{r}$, we obtain

$$
\mathcal{L}_{K}=-T \Omega_{2} R^{2}+T \Omega_{2} R \tilde{r}\left(2+\dot{\phi}^{2} R^{2}\right)-T \Omega_{2} \tilde{r}^{2}\left(\dot{\phi}^{4} R^{4}+\frac{5}{2} \dot{\phi}^{2} R^{2}+1\right),
$$

and

$$
\mathcal{L}_{B}=\Omega_{4} B R^{4} \dot{\phi}\left(1-3 \frac{\tilde{r}}{R}+3 \frac{\tilde{r}^{2}}{R^{2}}\right)=N \dot{\phi}-R \tilde{r} \frac{3 N}{R^{3}} \dot{\phi} R+\tilde{r}^{2} \frac{3 N}{R^{3}} \dot{\phi} R .
$$

Using $N / R^{3}=T \Omega_{2}$, the total Lagrangian becomes

$\mathcal{L}=-T \Omega_{2} R^{2}+N \dot{\phi}+T \Omega_{2} R \tilde{r}\left(2-3 \dot{\phi} R+\dot{\phi}^{2} R^{2}\right)-T \Omega_{2} \tilde{r}^{2}\left(\dot{\phi}^{4} R^{4}+\frac{5}{2} \dot{\phi}^{2} R^{2}-3 \dot{\phi} R+1\right)$.

There is an extremum at $\tilde{r}=0$ provided that

$$
2-3 \dot{\phi} R+\dot{\phi}^{2} R^{2}=0
$$

This can be achieved if the velocity $\dot{\phi} R=1$. Thus, when the size of the membrane is $R$ its center of mass moves with the speed of light. Furthermore, the extremum is stable since

$$
\left.\frac{d^{2} V(r)}{d^{2} r}\right|_{r=R}>0
$$

Thus when the size of the membrane is $R$, the angular momentum has its maximum value $N$ and the energy is given by

$$
E=T \Omega_{2} R^{2}=\frac{N}{R}
$$

At the classical level, this is in exact agreement with the energy of a KaluzaKlein graviton having angular momentum $N$ about the sphere. When $N>>1$, the maximal angular momentum is large and the classical formula for the energy agrees with the BPS bound for the energy given the angular momentum. Quantum corrections are suppressed by $1 / N$. The Kaluza-Klein graviton is a BPS state and its energy should not change under the process of blowing up into a membrane. Again, the size of the brane is determined by the angular momentum. Since the maximum size a brane can have is $R$, there is a maximum angular momentum as predicted by the dual conformal field theory. The fact that the energy of the brane 
agrees with the BPS formula for given angular momentum is a non-trivial test of our model.

We also note that there is a minimum of the energy at $r=0$ as well. Classically, it corresponds to a massless particle moving around the equator with angular momentum $L{ }^{25}$ Such a solution is singular from the perspective of the gravitational field equations since for angular momenta of order $N$, it represents a huge energy (of order $N^{2 / 3}$ ) concentrated at a point. Therefore it is subject to uncontrolled quantum corrections. In particular, there are quantum corrections proportional to powers of the momentum times the flux density, which are large at angular momenta of order $N$.

We have shown in this chapter that such a singular solution can be resolved by blowing into a smooth macroscopic membrane of size $(L / N) R$. Our classical analysis is expected to be valid for the large membrane. The smooth membrane solution certainly has much more nearby phase space and as a result the true quantum ground state will be overwhelmingly supported at the membrane solution. We believe that this is another example of how string/ $M$-theory resolves singularities of the type studied rececently in ref. [117].

\section{2. $A d S_{5} \times S^{5}$}

The extension of our analysis to the other two maximally supersymmetric cases is straightforward and we will be much less explicit. Consider the case of $A d S_{5} \times S^{5}$ first. The radius of the five sphere is given by

$$
R=\left(4 \pi g_{s} N\right)^{\frac{1}{4}} l_{s}
$$

where $g_{s}, l_{s}$ are the string coupling constant and string length scale and $N$ is the number of units of five-form flux on the sphere. We take $N$ large keeping $g_{s} N$ fixed and large.

In type IIB string theory on $A d S_{5} \times S^{5}$ with $N>>1$, the maximum angular momentum of a BPS particle on the $S^{5}$ is $N$ [111]. From the gauge theory perspective, this can be seen from the fact that one builds up such states by a single trace of the $N \times N$ scalars in the $\mathbf{6}$ of $S O(6)$. The largest representation of $S O(6)$ one can build in this way is the spin- $N$ representation, $\operatorname{Sym}^{N} \mathbf{6}$.

25 We thank Sunny Itzhaki for discussions of this point. 
From our perspective this is because the particle moving on the sphere expands into a spherical D3-brane. In this case we present only the exact classical analysis of the D3-brane wrapping an $S^{3}$ that moves in $S^{5}$. The bosonic Lagrangian is

$$
\mathcal{L}=\mathcal{L}_{D B I}+\mathcal{L}_{C S}=-T_{D 3} \Omega_{3} r^{3} \sqrt{1-\left(R^{2}-r^{2}\right) \dot{\phi}^{2}}+\dot{\phi} N \frac{r^{4}}{R^{4}} .
$$

The tension of the D3-brane is

$$
T_{D 3}=\frac{1}{(2 \pi)^{3} l_{s}^{4} g_{s}} .
$$

We will use the relation

$$
T_{D 3} \Omega_{3}=\frac{N}{R^{4}} .
$$

The angular momentum in terms of $\dot{\phi}$ is

$$
L=\frac{m \dot{\phi}\left(R^{2}-r^{2}\right)}{\sqrt{1-\dot{\phi}^{2}\left(R^{2}-r^{2}\right)}}+N \frac{r^{4}}{R^{4}}
$$

where $m=T_{D 3} \Omega_{3} r^{3}=\left(N / R^{4}\right) r^{3}$. Again we see that the angular momentum is bounded by $N$ since $0 \leq r \leq R$ and $0 \leq \dot{\phi} R \leq 1$. The energy is

$$
E=\sqrt{m^{2}+\frac{\left(L-N r^{4} / R^{4}\right)^{2}}{R^{2}-r^{2}}} .
$$

Varying the energy with respect to $r$ at fixed $L$, we find in this case a stable minimum when

$$
r^{2}=\frac{L}{N} R^{2}
$$

The value of the energy at this minimum again matches the BPS bound when $L$ is large, for $N>>1$ :

$$
E=\frac{L}{R} .
$$

This is strong evidence that at any appreciable momentum, at least at the (semi-) classical level, the good description of Kaluza-Klein gravitons is in terms of branes, rather than fundamental strings. From the dual CFT, we know that there is a unique BPS state with these quantum numbers; consistency with the exclusion principle implies that it is the one described by the spherical brane. 


\section{3. $A d S_{4} \times S^{7}$}

In this case, we expect the graviton to expand into an M5-brane which is an $S^{5} \subset S^{7}$. The radius of the sphere is given by

$$
R=\left(2^{5} \pi^{2} N\right)^{\frac{1}{6}} l_{p}
$$

The tension of the 5-brane is given by

$$
T=\frac{1}{(2 \pi)^{5} l_{p}^{6}}
$$

and we have the relation

$$
m=T \Omega_{5} r^{5}=\frac{N}{R^{6}} r^{5} .
$$

The Lagrangian is

$$
\mathcal{L}=-T \Omega_{5} r^{5} \sqrt{1-\left(R^{2}-r^{2}\right) \dot{\phi}^{2}}+\dot{\phi} N \frac{r^{6}}{R^{6}}
$$

The angular momentum in terms of $\dot{\phi}$ is

$$
L=\frac{m \dot{\phi}\left(R^{2}-r^{2}\right)}{\sqrt{1-\dot{\phi}^{2}\left(R^{2}-r^{2}\right)}}+N \frac{r^{6}}{R^{6}}
$$

The energy is

$$
E=\sqrt{m^{2}+\frac{\left(L-N r^{6} / R^{6}\right)^{2}}{R^{2}-r^{2}}} .
$$

Varying the energy with respect to $r$ at fixed $L$, we find in this case a stable minimum when

$$
r^{4}=\frac{L}{N} R^{4}
$$

The value of the energy at this minimum again matches the BPS bound when $L$ is large:

$$
E=\frac{L}{R}
$$




\subsection{Remarks about $A d S_{3}$}

We will conclude this section with some comments about $A d S_{3} \times S^{3} \times M_{4}$. This case is distinguished in several ways.

First, it is not clear into what the graviton should expand. Consider the geometry built from the D1-D5 system with $Q_{1}$ D-strings and $Q_{5}$ five-branes. The stringy exclusion bound on the angular momentum is $L \leq Q_{1} Q_{5}$. The graviton is expected to blow up into a circular string moving on the $S^{3}$, but should it be a D5-brane wrapped on the four-manifold, or a D-string?

Secondly, the energetic considerations degenerate in this case. If we assume for argument's sake that the graviton blows up into either a D-string or wrapped fivebrane which is a circle on the $S^{3}$, we find that the energy at fixed $L$ has no nontrivial minimum. Considering some incarnation of the "fractional strings" of [118] does not help.

It may help to consider the S-dual situation of the F1-NS5 system. In this case, the dynamics of fundamental strings on the relevant sphere are described by a level- $Q_{5} S U(2) \mathrm{WZW}$ model. One expects the exclusion principle to be related to the affine cutoff on $S U(2)$ representations. Finally, a clarification of this case should match the result of [119] that the exclusion bound occurs at the critical value of the energy for black hole formation. A better understanding remains for future work.

\section{Conclusions}

Physics in non-commutative spaces is characterized by a simple signature - the increase of size on systems with increasing momentum. In this chapter we have seen that the motion of massless quanta on the $S$ factor of $A d S_{n} \times S^{m}$ has exactly this behavior. The massless particle blows up into a spherical brane of dimensionality $m-2$ whose radius increases with increasing momentum. Eventually the radius of the blown up brane becomes equal to the radius of the sphere that contains it. It can no longer grow and the spectrum is terminated. This is the origin of the stringy

exclusion principle. Thus we see one more piece of evidence for non-commutativity of space in quantum gravity. 


\section{Acknowledgments}

We would like to thank Allan Adams, Micha Berkooz, Raphael Bousso, Sumit

Das, Antal Jevicki, Jason Prince Kumar, Albion Lawrence, Alec Matusis, and Joe

Polchinski for conversations. We are especially grateful to Joe Polchinski for ex-

plaining to us the flat-space energetics. We thank Hirosi Ooguri and Sunny Itzhaki

for comments about the first version. We would also like to thank Steve Shenker

for help with the title. The work of JM is supported in part by the Department of

Defense NDSEG fellowship program. The work of LS and NT is supported in part

by NSF grant 9870115 . 


\section{Chapter 6: M-theory on Manifolds of $G_{2}$ Holonomy and Type IIA Orientifolds}

We demonstrate that M-theory compactifications on 7-manifolds of $G_{2}$ holonomy, which yield $4 \mathrm{~d} \mathcal{N}=1$ supersymmetric systems, often admit at special loci in their moduli space a description as type IIA orientifolds. In this way, we are able to find new dualities of special IIA orientifolds, including dualities which relate orientifolds of IIA strings on manifolds of different topology with different numbers of wrapped D-branes. We also discuss models which incorporate, in a natural way, compact embeddings of gauge theory/gravity dualities similar to those studied in the recent work of Atiyah, Maldacena and Vafa.

\section{Introduction}

Compactifications of M-theory and string theory down to $4 \mathrm{~d} \mathcal{N}=1$ supersymmetry are of obvious interest. The reduced supersymmetry is probably necessary for any contact with real world physics. It also allows for richer phenomena than extended supersymmetry and so provides a nice playground for theorists.

Generic methods of constructing such models include compactifying the heterotic string on Calabi-Yau threefolds, F-theory on Calabi-Yau fourfolds, and Mtheory on 7-manifolds of $G_{2}$ holonomy. Although many basic facts about all of these classes of compactifications remain mysterious, perhaps the least is known about the last class, since at least the others are amenable to attack using techniques of complex geometry.

A large class of compact 7-manifolds with $G_{2}$ holonomy was constructed by Joyce $[121,122]$. In this note, we make the simple observation that M-theory compactified on many of these spaces admits, at special loci in its moduli space, a description as an orientifold of type IIA string theory compactified on a Calabi-Yau

The material in Chapter 5 appeared in "M-theory on Manifolds of $G_{2}$ Holonomy and Type IIA Orientifolds" with Shamit Kachru [120] and is reprinted with permission of JHEP. 
threefold. ${ }^{26}$ This is reminiscent of the fact that F-theory models can be reformulated, at special loci in their moduli space, as type IIB orientifolds [124,125].

There are several different reasons this observation can be useful. On the one hand, the orientifolds we discuss have a rather simple, solvable structure, and so provide a very concrete handle on these models at some special points in their moduli space. On the other hand, as we will show, a given $G_{2}$ space can admit different type IIA orientifold limits. Thus, by studying limit points in the moduli space of $G_{2}$ compactifications, we learn about non-perturbative dualities of IIA compactifications with $\mathcal{N}=1$ supersymmetry. In particular, we exhibit an example where orientifolds of type IIA on Calabi-Yau spaces of different topology (and with different numbers of D-branes and orientifold planes) are dual to each other. On yet a third hand, our construction "globalizes" interesting gauge theory/gravity dualities similar to those encapsulated in the local models of [126] and [127].

In $\S 2$, we introduce the $G_{2}$ manifold $X$ which will be our focus in part of this note. In $\S 3$, we show that various limit points in the moduli space of M-theory on $X$ are well described by IIA orientifolds. This observation allows us to find nonperturbative duality symmetries of these orientifolds. In $\S 4$ we make some remarks about the extent to which our analysis generalizes to other $G_{2}$ spaces, and also provide a simple proof that a large class of IIA orientifolds should have an "Mtheory lift" to $G_{2}$ compactifications. In $\S 5$ we explain how gauge theory/gravity dualities analogous to those discussed in $[126,127]$ naturally arise in simple examples of compact $G_{2}$ manifolds and the related IIA orientifolds. We conclude in $\S 6$ by mentioning some interesting directions for further study.

Several papers analyzing various related aspects of M-theory on $G_{2}$ manifolds have appeared recently. Dual descriptions of $\mathcal{N}=1$ gauge theories using such spaces have been discussed in $[126,127,128,129,130,131]$, while [132] discusses a general relationship between certain classes of wrapped branes and geometries with exceptional holonomy. Earlier work on this subject appears in [133]. Phase transitions between topologically distinct $G_{2}$ compactifications were described in [134].

26 We ignore the possibility of a membrane instanton generated superpotential [123] in most of our discussion. If such a potential exists, it would provide a potential barrier between the large-volume M-theory and perturbative type IIA limits. Our successful comparison of these limits in many cases suggests that either such a potential is absent, or the interpolation over the potential barrier is nevertheless physically meaningful. 


\section{The Manifold $X$}

A basic example of a compact 7-manifold of $G_{2}$ holonomy is the manifold $X$ considered by Joyce in [121]. It is constructed as a toroidal orbifold. Let $x_{1}, \cdots, x_{7}$ parametrize a square $T^{7}$ which is a product of seven circles of radii $r_{1}, \cdots, r_{7}$. Define $X$ as the (desingularization of the) quotient of this $T^{7}$ by the $Z_{2}^{3}$ group with generators

$$
\begin{gathered}
\alpha\left(x_{i}\right)=\left(-x_{1},-x_{2},-x_{3},-x_{4}, x_{5}, x_{6}, x_{7}\right) \\
\beta\left(x_{i}\right)=\left(-x_{1}, 1 / 2-x_{2}, x_{3}, x_{4},-x_{5},-x_{6}, x_{7}\right) \\
\gamma\left(x_{i}\right)=\left(1 / 2-x_{1}, x_{2}, 1 / 2-x_{3}, x_{4},-x_{5}, x_{6},-x_{7}\right)
\end{gathered}
$$

where $1 / 2$ denotes a shift of order 2 around the circle. Then as demonstrated in [121], $X$ has betti numbers $b_{2}(X)=12, b_{3}(X)=43$. Therefore, M-theory compactification on $X$ gives rise to a $4 \mathrm{~d} \mathcal{N}=1$ supersymmetric low-energy theory with (generically) 12 abelian vector multiplets and 43 chiral multiplet moduli.

It may be useful to review the origin of the various cohomology classes on $X$ here. None of the two-forms and seven of the three-forms on $T^{7}$ are invariant under the action of $\langle\alpha, \beta, \gamma\rangle \cdot{ }^{27}$ In addition, each of the generators fixes $16 T^{3} \mathrm{~s}$ on $T^{7}$; however e.g. the $16 T^{3}$ s fixed by $\alpha$ are identified by the group $\langle\beta, \gamma\rangle$ to yield 4 on the quotient $X$, and the fixed tori of $\beta$ and $\gamma$ undergo a similar fate. The local form of the singularities at the fixed $T^{3} \mathrm{~s}$ is $R^{4} / Z_{2} \times T^{3}$, and resolving each of these yields a two-form and three three-forms. Since there are 12 such fixed tori on $X$, after desingularizing one has the stated betti numbers.

\section{Orientifold Limits of $X$}

In this section, we demonstrate that $X$ has several different IIA orientifold limits in its moduli space. This in particular tells us that the different orientifolds are related to one another by various dualities.

$27\langle\cdots\rangle$ denotes "the group generated by $\cdots . "$ 


\subsection{Orientifold $A$}

We start by viewing $x_{7}$ as the "M-theory circle," or the eleventh dimension. Then in the limit of small $r_{7}$, we should be able to get an effective IIA description of M-theory on $X$. Denote by $\alpha^{*}$ the action of $\alpha$ restricted to the $T^{6}$ with coordinates $x_{1}, \cdots, x_{6}$. Then since $\alpha$ and $\beta$ don't act on the M-theory circle anyway, in the limit of small $x_{7}$ they simply induce identifications on the $T^{6}$ visible to the type IIA string. It is therefore propagating on the Calabi-Yau space $N=T^{6} /\left\langle\alpha^{*}, \beta^{*}\right\rangle$.

However, $\gamma$ also acts on the M-theory circle. Using the results of [135], it follows that the action of $\gamma$ (inversion of the M-theory circle and three other coordinates) is mapped in the IIA theory to $w=(-1)^{F_{L}} \Omega \gamma^{*}$. Thus, IIA string theory on the orientifold of $N$ by $w$ should govern M-theory in the limit of small radius for $x_{7}$. Let us call this model orientifold $\mathrm{A}$.

To check this conjecture, let us try to match up the counting of fields. $N$ has hodge numbers $h^{1,1}=19, h^{2,1}=19$. So IIA string theory on $N$ yields an $\mathcal{N}=2$ supersymmetric 4d theory with 20 hypermultiplets (including the dilaton) and 19 vector multiplets. Now, projecting by $w$ has the following effect (see e.g. [136]). Each of the 20 hypermultiplets is projected down to a chiral multiplet. The vector multiplets (which came from the Kähler moduli) are more subtle: those which come from $(1,1)$ forms invariant under $w$ give rise to $\mathcal{N}=1$ vector multiplets, while those which are anti - invariant under $w$ give rise to chiral multiplets. It is easy to convince oneself that the untwisted $(1,1)$ forms on $N$ are anti-invariant, while the 16 twisted $(1,1)$ forms split into \pm eigenspaces of equal size. Therefore, the Kähler moduli contribute 11 chiral multiplets and 8 vector multiplets.

This accounts for 31 chiral multiplets and 8 abelian vectors so far. However, we must also take into account the fixed points of the $w$ action. $\gamma^{*}$ acts with 8 fixed loci on $T^{6}$. Identification by $\langle\beta, \gamma\rangle$ reduces this to 2 fixed loci; a neighborhood of each in the threefold is of the form $R^{3} / Z_{2} \times T^{3}$. Therefore, there are orientifold six-planes wrapping each of these $T^{3} \mathrm{~S}$ (as in [135]).

By the normal tadpole cancellation considerations, we must introduce $2 D 6$ branes for each $O 6$ plane. Hence, we introduce a total of $4 D 6$ branes wrapping $T^{3} \mathrm{~s}$ in this model. Each of the $D 6$ branes comes with a $U(1)$ vector multiplet and 3

chiral multiplet moduli (coming from the Wilson lines on the $T^{3}$, together with the moduli of the three-cycle in $N$ ). So the $D 6$ branes contribute a total of 12 chiral multiplet moduli and 4 vector multiplets to the low-energy theory. 
Totalling up the spectrum, we find that orientifold A (at generic points in its moduli space) has 43 chiral multiplet moduli and 12 vector multiplets, just as it must to match the spectrum of M-theory on $X$. At special points in moduli space when the $D 6$ branes coincide, one achieves enhanced gauge symmetries, which come from geometrical singularities in the M-theory picture [135].

\subsection{Orientifold $B$}

It is of course also possible to view other circles as the M-theory circle. For instance, we could take $x_{4}$ to be the M-theory circle. However, repeating the same logic as in $\S 3.1$, we would find that we again arrive (in the small $r_{4}$ limit) at an orientifold (which we could call orientifold B) of type IIA string theory on the Calabi-Yau orbifold with hodge numbers $h^{1,1}=19, h^{2,1}=19$, and we again have to introduce the same numbers of $D 6$ branes.

The role of the dilaton in orientifold $A$ is played by a geometrical modulus in orientifold $B$, and vice-versa. However, they are really compactifications on the same target space. This means that the IIA theory on the orientifold of $N$ by $w$, discussed in $\S 3.1$, has a sort of $S-T$ exchange symmetry, where $S$ is the dilaton chiral multiplet and $T$ is the chiral multiplet containing the radius of $x_{4}$. This way of seeing the $S-T$ exchange symmetry of these orientifold models is analogous to the way that the $S-T$ exchange symmetry [137] of the main heterotic string examples in [138] can be understood as arising from the existence of multiple $K 3$ fibrations in the type II-dual Calabi-Yau compactifications [139,140].

In fact, this model enjoys more symmetry than just a single $S-T$ duality; one could equivalently consider the $x_{6}$ circle to be the M-theory circle, with the same results, yielding a sort of $S-T-U$ triality symmetry.

\subsection{Orientifold $C$}

A more interesting possibility is to interpret $x_{5}$ as the M-theory circle. Then acting on the $T^{6}$ coordinates $x_{1}, x_{2}, x_{3}, x_{4}, x_{6}, x_{7}$, we have

$$
\begin{gathered}
\alpha\left(x_{i}\right)=\left(-x_{1},-x_{2},-x_{3},-x_{4}, x_{6}, x_{7}\right) \\
\beta \gamma\left(x_{i}\right)=\left(x_{1}+1 / 2,1 / 2-x_{2},-x_{3}, x_{4},-x_{6},-x_{7}\right)
\end{gathered}
$$


The manifold $N^{\prime}=T^{6} /\langle\alpha, \beta \gamma\rangle$ is then a Calabi-Yau threefold with hodge numbers $h^{1,1}=11, h^{2,1}=11$. In particular, it is topologically distinct from the threefold $N$ which appeared in $\S 3.1$ and $\S 3.2$.

Define $u$ to be the composition of $(-1)^{F_{L}} \Omega$ with the action of $\gamma$ on the $T^{6}$ coordinates. Then in the limit of small $r_{5}$, M-theory on $X$ should be well described by IIA theory on the orientifold of $N^{\prime}$ by $u$, which we will call orientifold C.

Let's check that the spectrum matches our expectations. $v$, the composition of $(-1)^{F_{L}} \Omega$ with the action of $\beta$ on the $T^{6}$ coordinates, arises upon composing $u$ with elements of the orbifold group. Both $u$ and $v$ act with fixed loci on $N^{\prime}$. Each has 8 fixed $T^{3} \mathrm{~s}$ in the $T^{6}$, which descend to 2 fixed $T^{3} \mathrm{~s}$ in the orbifold $N^{\prime}$. Therefore, one has to introduce four $O 6$ planes, and 8 wrapped $D 6$ branes are required to cancel the RR tadpoles. These give rise to 8 abelian vector multiplets and 24 chiral multiplets, at generic points in moduli space.

The projection of the spectrum of IIA on $N^{\prime}$ can be done as before. Once again, half of the 8 twisted $(1,1)$ forms are invariant under the orientifold action, while the other half (and the untwisted $(1,1)$ forms) are anti-invariant. So we get 4 vectors and 7 chirals from the $(1,1)$ forms; adding in the 12 chirals descending from the $\mathcal{N}=2$ hypermultiplets, we indeed find a total of 43 chiral multiplets and 12 vectors.

In this orientifold $\mathrm{C}$ picture, the radii $r_{4}, r_{6}, r_{7}$ which are related (up to triality) to the dilaton in the pictures of $\S 3.1$ and $\S 3.2$ are all geometrical moduli of the IIA compactification, while $r_{5}$ (which is geometrized in orientifolds $\mathrm{A}, \mathrm{B}$ ) is playing the role of the dilaton. This gives an example of a strong/weak duality between IIA orientifolds of topologically distinct Calabi-Yau spaces, with different numbers of space-filling D-branes and orientifold planes.

\section{Generalization to Other Models}

In this section, we generalize our results in two directions. We first show that a large class of $G_{2}$ spaces should similarly have orientifold limits. We then take the opposite approach, and prove that a wide class of IIA orientifolds have an M-theory lift to $G_{2}$ compactifications. 


\subsection{Other Classes of $G_{2}$ Manifolds}

Beyond the toroidal orbifold constructions of Joyce, there are other methods of constructing $G_{2}$ holonomy spaces which are amenable to an orientifold interpretation.

\section{Barely $G_{2}$ Manifolds}

Harvey and Moore defined "barely $G_{2}$ manifolds" as quotients of the form $X=$ $\left(Y \times S^{1}\right) / Z_{2}$, where $Y$ is a Calabi-Yau threefold and the $Z_{2}$ action is a composition of a freely acting antiholomorphic involution $\sigma$ on $Y$ with inversion on the circle factor $x_{7}$ [123]. These are of course a special case of a more general construction which should arise when $\sigma$ has fixed points $[121,122]$.

For the barely $G_{2}$ spaces, it turns out that

$$
\begin{gathered}
H^{3}(X)=H^{2}(Y)^{-}+H^{3}(Y)^{+} \\
H^{2}(X)=H^{2}(Y)^{+}
\end{gathered}
$$

where \pm refer to eigenvalues under the action of $\sigma$ on $Y$. For simple examples which come from hypersurfaces in toric varieties, one simply keeps the complex structure deformations which preserve the real structure (i.e. defining equations with real coefficients), so $H^{3}(Y)$ has \pm eigenspaces of equal dimension. For such examples, we find $n_{C}=h^{1,1}(Y)^{-}+h^{2,1}(Y)+1$ chiral multiplets and $n_{V}=h^{1,1}(Y)^{+}$vector multiplets in M-theory on $X$.

As one shrinks the radius $r_{7}$ of the $S^{1}$, one should obtain a IIA description. Indeed, since the $Z_{2}$ above acts with an inversion on $x_{7}$, we should expect that the orientifold of IIA on $Y$ by $(-1)^{F_{L}} \Omega$ composed with $\sigma$ arises in this limit. It follows from the general considerations of [136] (as discussed in §3) that the spectrum of this type IIA orientifold agrees with the M-theory spectrum.

\section{Cases with Fixed Points}

It is attractive to speculate about generalizations of the previous case to cases where $\sigma$ acts on $Y$ with fixed points. On general grounds, the fixed point locus $\Sigma \subset Y$ will be a special Lagrangian (sL) three-cycle (or several, in which case one should repeat the discussion below for each component). It is not known in generality how to resolve the singularities in this case to obtain a smooth metric of $G_{2}$ holonomy. 
However, the existence of an orientifold limit leads to a very natural conjecture. Shrinking the $x_{7}$ circle again, we find a IIA model which should have an $O 6$ plane and two $D 6$ branes wrapping $\Sigma$. For $\Sigma$ special Lagrangian, a D6 brane wrapping $\Sigma$ gives rise to a single $\mathcal{N}=1$ vector multiplet and $b_{1}(\Sigma)$ chiral multiplets in spacetime. Therefore, we expect that there will be 2 vectors and $2 b_{1}(\Sigma)$ chiral multiplets associated with the $D 6$ branes in this limit. When the $D 6$ branes are coincident, the model has enhanced gauge symmetry (which shows up in the Mtheory as the singularity of the $G_{2}$ space related to the fixed points of $\sigma$ ). For $b_{1}(\Sigma)>0$, one can move in the $D 6$ brane moduli space to remove the enhanced gauge symmetry. It is then attractive to conjecture that in the M-theory picture, $b_{1}(\Sigma)>0$ is a condition that allows the singularities of this class of $G_{2}$ orbifolds to be repaired, and that furthermore resolving the singularity gives rise to precisely two elements of $b_{2}(X)$ and $2 b_{1}(\Sigma)$ elements of $b_{3}(X) .^{28}$

\section{2. "All" Orientifolds of type IIA on CY have a $G_{2}$ Limit}

Suppose we have a IIA orientifold which gives rise to a four dimensional $\mathcal{N}=1$ supersymmetric theory. For simplicity, let us first restrict ourselves to orientifolds of tori. The orbifold part of the orientifold group must have (at most) $S U(3)$ holonomy, to preserve (at least) $4 \mathrm{~d} \mathcal{N}=2$ supersymmetry. $^{29}$ Let us assume we are in the most generic case, so that it preserves precisely $\mathcal{N}=2$ supersymmetry. Denote the full orientifold group by

$$
\mathcal{G}=\Gamma_{1} \times(-1)^{F_{L}} \Omega \Gamma_{2}
$$

The $(-1)^{F_{L}}$ is present because we choose, as in [135], a convention where reflection on three circles must be accompanied by a $(-1)^{F_{L}}$ to preserve supersymmetry in the IIA theory, and we will show momentarily that all elements of $\Gamma_{2}$ must reflect precisely three circles of the $T^{6}$. With these assumptions, $T^{6} / \Gamma_{1}$ alone is CalabiYau, and so has a holomorphic three-form $\Omega^{(3,0)}$ and a Kähler form $J$.

28 This could be related to Condition 4.3.1 in [122], which was stated without proof to be an important condition in resolving singularities of this sort.

29 This is because there are no geometric compactifications of IIA down to $4 \mathrm{~d}$ which preserve precisely $4 \mathrm{~d} \mathcal{N}=1$ supersymmetry. 
Now, consider the $(-1)^{F_{L}} \Omega \Gamma_{2}$ part of the group. Any element $(-1)^{F_{L}} \Omega g_{2}$ of this part must have $g_{2}$ reversing the orientation of the $6 \mathrm{~d}$ target, or it cannot be a symmetry of the IIA theory. So we know a few things about the $g_{2}$ action:

i) $g_{2}$ maps $J$ to $-J$ (orientation reversal) and

ii) $g_{2}$ maps $\Omega^{(3,0)}$ to $\bar{\Omega}^{(0,3)}$. Notice that this implies that $g_{2}$ reflects precisely three circles of the $T^{6} / \Gamma_{1}$, as required above.

In $i i$ ), we are using the fact that to preserve one supersymmetry, some linear combination of the killing spinors must be preserved. This means that $g_{2}$ either preserves the holomorphic and anti-holomorphic three-form individually, or at least preserves a linear combination. But since $\Omega^{(3,0)} \wedge \bar{\Omega}^{(0,3)} \sim J \wedge J \wedge J$, by $i$ ) above $g_{2}$ must permute the two. One might worry that $g_{2}$ could act with a phase in relating $\Omega^{(3,0)}$ to its conjugate; but all $g_{2} \subset \Gamma_{2}$ which exchange $\Omega^{(3,0)}$ and its conjugate would have to have the same phase to preserve $\mathcal{N}=1$ supersymmetry. It can then be redefined to 1 by a phase rotation of $\Omega^{(3,0)}$.

To proceed, we add a seventh M-theory circle $x_{7}$. Define the new group $\tilde{\mathcal{G}}$, which acts on $T^{7}$, as follows: take each element of $\mathcal{G}$ and replace $(-1)^{F_{L}} \Omega$ anywhere it appears with inversion of the $x_{7}$ coordinate (while elements which don't include a $(-1)^{F_{L}} \Omega$ act trivially on the $x_{7}$ coordinate). This will not change anything about elements of $\Gamma_{1}$ (since the minus sign on $x_{7}$ will cancel in the product of two $(-1)^{F_{L}} \Omega \Gamma_{2}$ elements). However, under assumptions i) and ii) above, the three-form

$$
\Phi=J \wedge d x_{7}+\operatorname{Re}\left[\Omega^{(3,0)}\right]
$$

is preserved by the whole (now orbifold) group $\tilde{\mathcal{G}}$ acting on $T^{7}$. This form is preserved by a $G_{2}$ subgroup of $G L(7, R)$ [122]. This is sufficient to prove that the resulting manifold is a $G_{2}$ space.

It is clear that this argument is more generically applicable to supersymmetric models which are not toroidal orientifolds. One could replace the $T^{6}$ in the IIA theory with any manifold $M$, use the fact that $M / \Gamma_{1}$ should be Calabi-Yau to preserve supersymmetry in the IIA theory, and apply the same logic. 


\section{The Case of the Disappearing Orientifold}

Recent work has made it clear that gauge dynamics on wrapped $D 6$ branes (or arising from singular M-theory geometries) can often be encoded by smooth geometries in a "dual" gravity description [126]. The gauge dynamics is then encoded in appropriate RR-fluxes, or in changes of the behavior of the M-theory three-form $C$ field, which (suitably interpreted) capture the low-energy physics of the gauge theory. In this section, we discuss examples of this phenomenon which arise in string/M-theory compactifications in a natural way.

The most obvious source of consistent compact models with wrapped $D 6$ branes is the Calabi-Yau orientifolds discussed here. The components of the orientifold fixed locus provide sL three-cycles $\Sigma$, which are wrapped by orientifold planes and $D 6$ branes. In fact, examples of sL cycles $\Sigma$ which arise in this way were studied in $[18,39]$ precisely with the motivation of understanding the dynamics on the worldvolumes of such wrapped $D 6$ branes.

One interesting fact (which had perplexed some of the authors of [18,39] for some time) is that it is possible for the fixed locus of an anti-holomorphic involution to disappear as the complex structure of the Calabi-Yau varies; and the relevant complex structure moduli survive in the orientifold models. This fact was used in [39] to identify D6 branes on such real slices as mirror to D5 branes on vanishing holomorphic curves. However, it raises the question: if one continues past the point in moduli space where the fixed locus disappears (so there is no orientifold plane, and no need to introduce $D 6$ branes), where has the information about the gauge theory on the $D 6$ branes gone? The gauge theory/gravity dualities relevant to this situation were studied in $[126,127]$, and provide the answer to this question.

Let us illustrate this with a simple example. The easiest examples discussed in [39] basically involve a sL three-cycle which is the fixed locus of a real involution and which collapses at a conifold singularity. So locally, the geometry of the compact Calabi-Yau $M$ looks like

$$
z_{1}^{2}+z_{2}^{2}+z_{3}^{2}+z_{4}^{2}=\mu
$$

where $\mu$ is chosen to be a positive real parameter. Then under the involution

$$
\mathcal{I}: z_{i} \rightarrow \bar{z}_{i}
$$


the fixed point locus $\Sigma^{+}$is the three-sphere

$$
\Sigma^{+}: \sum_{i=1}^{4} x_{i}^{2}=\mu
$$

where $z_{i}=x_{i}+i y_{i}$.

We can embed this situation in a $G_{2}$ manifold as in $\S 4$. , where the $G_{2}$ manifold $X$ is of the form $\left(M \times S^{1}\right) / \sigma$. The $Z_{2}$ symmetry $\sigma$ acts by $\mathcal{I}$ combined with inversion on the M-theory circle, $x_{7} \rightarrow-x_{7}$. Then for $\mu>0$, the fixed point loci of $\sigma$, which consist of copies of $\Sigma^{+}$at $x_{7}=0,1 / 2$, are actually $S^{3}$ s of $A_{1}$ singularities in $X$. This gives rise in M-theory on $X$ to two $4 \mathrm{~d}, \mathcal{N}=1$ pure $S U(2)$ gauge theories (with equal gauge couplings).

Now, consider taking $\mu$ through 0 . At $\mu \rightarrow 0$ there are collapsing associative three-cycles in $X$, and hence membrane instanton effects are expected to be large [123]. However, the sizes of the $S^{3}$ s come paired in chiral multiplets with periods of the three-form $C$ field over the $S^{3}$ s, and for generic values of this phase, there is no singularity in the physics - singularities in $\mathcal{N}=1$ moduli spaces happen at complex codimension one. Therefore, one can smoothly (in the physical sense) continue from $\mu>0$ to $\mu<0$. This raises a puzzle: the $S U(2)$ gauge groups present for $\mu>0$ have now disappeared, since the $Z_{2}$ symmetry $\sigma$ acts on $X$ without fixed points for $\mu<0$. However, the information about the gauge theory must be encoded somehow in the $\mu<0$ geometry.

The basic point is as in [126]. For $\mu<0$, one can still look for a homologically nontrivial three-sphere which membrane instantons can wrap. For instance, consider the locus of pure imaginary $z_{i}$, still at $x_{7}=0,1 / 2$. This is given by a three-sphere

$$
\sum_{i=1}^{4} y_{i}^{2}=-\mu
$$

which is orbifolded by the freely-acting $Z_{2}$ symmetry $y_{i} \rightarrow-y_{i}$. Call the resulting $\mathbb{R I}^{3} \Sigma^{-}$. It turns out that $\Sigma^{-}(-\mu)$ has exactly half the volume of $\Sigma^{+}(\mu)$, due to the orbifolding. These $\mathbb{R P}^{3}$ s are associative three-cycles in $X$ for $\mu<0$. However, as in [126], changing the period of the $C$ field on $\Sigma^{+}(\mu)$ by $2 \pi$, which is physically 
meaningless, corresponds to changing it by $\pi$ on $\Sigma^{-}(-\mu)$, due to the smaller volume. This ambiguity in the choice of phase for $\mu<0$ corresponds to the vacuum degeneracy due to the gaugino condensate in the gauge theory. ${ }^{30}$

In the IIA picture, with $x_{7}$ taken as the M-theory circle, this becomes an example where an orientifold plane and two $D 6$ branes, present for $\mu>0$, disappear as $\mu$ passes through 0 . This system has an $S O(4)$ gauge symmetry, and should give rise to multiple vacua after gaugino condensation, in agreement with the M-theory picture above. The phase ambiguity detected by membrane instantons in M-theory is detected by $D 2$ brane instantons in the string theory picture. This is in accord with our gauge theory intuition, since $D 2$ branes are the instantons of the $D 6$ brane gauge theory in the phase where the $D 6$ branes exist [141]. The fact that $\Sigma^{-}(-\mu)$ has half the volume of $\Sigma^{+}(\mu)$ then becomes the familiar fact that the superpotential from a gaugino condensate in $\mathcal{N}=1 S U(2)$ gauge theory looks like a "half-instanton effect."

More precisely, once we have compactified this setup, the superpotential we are discussing destabilizes the closed-string modulus $\mu$ (which is a parameter in the non-compact case). In our discussion here, we are imagining that we can hold $\mu$ fixed at various values, which is reasonable as long as the scale generated by the superpotential is parametrically smaller than the string/Planck scale. This is true for large enough $|\mu|$.

It is clear that the other examples of [39], which involve sL three-cycles $\Sigma$ with $b_{1}(\Sigma)>0$, could also be lifted in this way to find examples of M-theory "dualities" in gauge theories with adjoint matter. Some examples of this have already appeared in $[129]$.

\section{Discussion}

Little is known about M-theory compactification on spaces of $G_{2}$ holonomy. Naive extrapolation of the kinds of results that exist so far suggests that further

30 Notice that since the two associative $S^{3}$ s at $x_{7}=0,1 / 2$ are in the same homology class, their volumes (and the periods of the $C$-field) are the same. So although there are two $S U(2)$ s, the choice of phase in the two gaugino condensates is related - there is only a single $Z_{2}$ ambiguity. This carries over to the $I I A$ picture as well. 
study of the relationship between IIA orientifolds and M-theory compactifications could yield:

1) A large class of examples of non-perturbative dualities between orientifolds of type II compactifications on Calabi-Yau spaces of different topologies, with different numbers of space-filling D-branes.

2) New gauge theory/gravity dualities along the lines of [126], in a compact context (i.e., coupled to $4 \mathrm{~d}$ gravity).

3) Connections between the study of disc instanton effects in type II compactifications with branes (see e.g. $[18,39,142,143])$ and membrane instanton effects in M-theory [123]. The real involution of the CY pairs holomorphic discs [39] even when the sL three-cycle on which they end is deformed away from the real slice. In this way, pairs of discs times the M-theory circle form closed orbifold-invariant three-manifolds which membrane instantons can wrap. Similarly, $\mathbb{R}^{2}$ worldsheets with their crosscap on the real slice lift to orbifolds of membrane instantons on the M-theory circle times the covering sphere of the $\mathbb{R I}^{2}[127]$.

4) A good understanding of the new physics which arises at singularities of Mtheory on spaces of $G_{2}$ holonomy (some examples of this were discussed in [130]). It would be particularly interesting to find various singularities which correspond to chiral gauge theories. Perhaps these would provide a useful tool for the further exploration of chirality changing phase transitions [144].

5) A new window into type I compactifications. The type IIA orientifolds studied here are T-dual to type I string compactifications (roughly speaking, by T-duality on the $T^{3}$ fibers [145] of the Calabi-Yau space which is being orientifolded). Therefore, any insights gained about these models through their M-theory interpretation will carry over to the study of certain type I theories.

\section{Acknowledgements}

We would like to thank J. Polchinski and E. Silverstein for helpful discussions, and B. Acharya for pointing out a minor error in $\S 5$ of the first version of this note. This work was supported in part by NSF grant PHY-95-14797 and by the DOE under contract DOE-AC03-76SF00098. S.K. enjoyed the hospitality of the ITP Santa Barbara "M-theory" program while performing the work reported here, 
and was supported by the National Science Foundation under grant number PHY-

99-07949. The work of S.K. was supported in part by a David and Lucile Packard

Foundation Fellowship for Science and Engineering and an Alfred P. Sloan Foun-

dation Fellowship. The work of J. M. was supported in part by the Department of

Defense NDSEG Fellowship program. 


\section{Chapter 7: D(NA)-Branes}

We engineer a configuration of branes in type IIB string theory whose mechanical structure is that of a DNA molecule. We obtain it by considering a T-dual description of the quantum Hall soliton. Using a probe analysis, we investigate the dynamics of the system and show that it is stable against radial perturbations. We exercise a certain amount of restraint in discussing applications to biophysics.

\section{Introduction}

String theory - at present the only known consistent theory of quantum gravity - has undergone several major changes during its lifetime in terms of the way in which physicists look at it, and what they expect from it. Originally it was viewed as a possible theory of strong interactions, although the ineluctable appearance of a massless spin-2 state in its spectrum dampened the enthusiasm for the idea. When this phenomenon was later reinterpreted as a feature rather than a bug, with the spin-2 state incarnate as a graviton rather than an anomalously light hadron, the theory garnered a reputation as the leading candidate for a theory incorporating the phenomenon of gravity into quantum mechanics. The discovery of supersymmetry, a cornucopia of compactifications to four dimensions, and quasi-realistic gauge groups and matter content in the string framework only served to solidify the hegemony of strings over the field of theoretical high-energy physics.

Much of the recent excitement in string theory has come from an (often nonperturbative) understanding of many different backgrounds of the theory and the relations between them. The protean character of quantum states under the duality group of string theory is a source of much of the continuing fascination with the theory, and of the general sense that it has many depths yet to be explored. Indeed it seems that just about any quantum theory imaginable can be obtained

The material in this chapter appeared in "D(NA)-Branes" with Simeon Hellerman [146] and is reprinted with permission of JHEP. 
as a (low-energy or other) limit of the dynamics of the theory. Its shape-shifting capacity appears unlimited.

Most recently, the theory has been investigated because of the fact that one of its backgrounds seems to be able to reproduce the quantum Hall effect at low energies. In fact two logically separate dualities relating the Hall system to other theories of interest have emerged recently. The first [147] is a direct embedding of the system in string theory. The second [148] is an equivalence to a noncommutative gauge theory whose relation to string theory is less direct. This equivalence was recently sharpened in $[149,150]$.

In this chapter we describe a closely related system, obtained by considering the T-dual of (a nonabelian version of) the system in [147]. We show that the T-dual is a double helix, with fundamental string rungs connecting the two helices.

The plan of the chapter is as follows. In $\S 2$, we briefly review the construction of the quantum Hall soliton, and motivate an examination of the D(NA)-brane system by considering its T-dual. In $\S 3$ we use the Dirac-Born-Infeld/Wess-Zumino action on the lighter branes to find the equilibrium radius of the helix. In $\S 4$ we describe the low-energy dynamics of the system. In $\S 5$ we conclude by discussing the limitations of our calculation and ways in which it might be improved.

\section{The quantum Hall soliton and its DNA dual}

We briefly review the construction [147] of a quantum Hall-like system in type IIA string theory.

Begin with a set of $k$ sixbranes, spatially extended in the $x^{4}, \cdots, x^{9}$ directions, located at transverse position $x_{1}=x_{2}=x_{3}=0$. Surrounding these sixbranes we place a set of $n$ coincident twobranes - that is, twobranes whose location is defined by the sphere $x_{1}^{2}+x_{2}^{2}+x_{3}^{2}=R_{0}^{2}$, with $R_{0}$, the radius of the sphere, determined by the dynamics and specified later. When we say 'surrounding' we mean that the twobranes literally cannot be moved off to infinity without intersecting the sixbranes.

It is known [151] that for such nontrivially 'linked' configurations of D-branes in type II string theories there is an effect that causes fundamental strings to connect the two linked objects. Referred to as the 'Hanany-Witten' effect after its 
discoverers, this feature of brane dynamics can be understood from many different points of view, c.f. e.g. [152].

In order to stabilize the configuration one can dissolve $n$ zerobranes in the set of twobranes. There is a repulsive force between zerobranes and sixbranes at long distances, and so the system seeks its equilibrium radius, calculated in [147] to be $R_{0}=\left(\pi k^{\prime} / n\right)^{2 / 3} l_{s} / 2$.

The authors of [147] argued that the dynamics of this system were closely related to those of the quantum Hall effect, in that the string endpoints appeared in the gauge theory dynamics as charges in the fundamental representation (in [147] only a single twobrane was considered, and so the string endpoints were simply electric charges) and the dissolved zerobranes were units of magnetic flux. For a single twobrane the 'filling fraction' of the system was simply $k / k^{\prime}$, the ratio of the density of electrons to the density of flux.

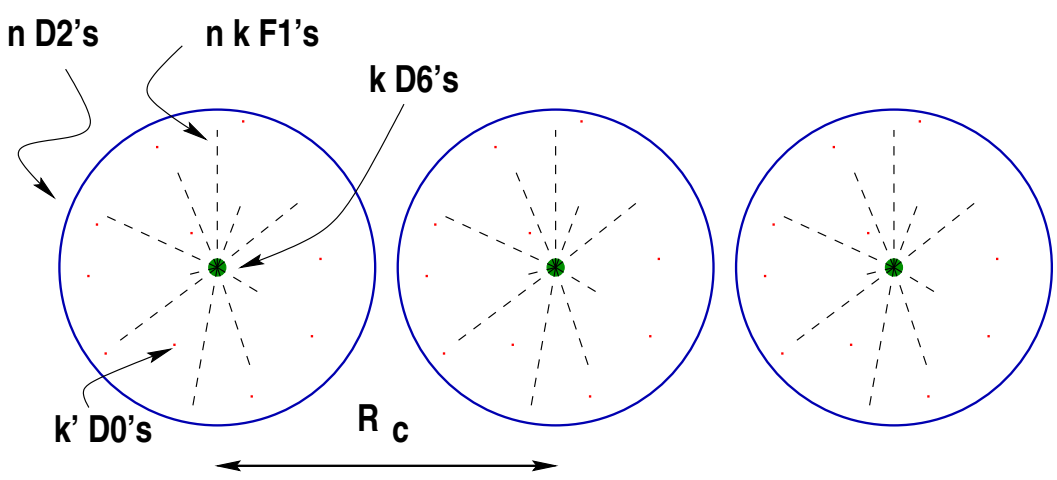

Fig. 9: The periodic array of quantum Hall solitons before the GregoryLaflamme transition.

Now suppose one compactifies the system along the $x_{3}$ direction with radius $R_{c}$. For $R_{c}>>R_{0}$ we can think of the periodically identified configuration as an infinite array of twobrane spheres surrounding an array of sixbranes as in fig. $9^{31}$. We expect, however, that as we reduce the compactification radius until $R_{c} \sim R_{0}$, the system will develop an instability (closely related to the Gregory-Laflamme instability [153]) towards a merger of the twobranes into a cylinder, periodically identified in the compact direction.

31 Strictly speaking as soon as one compactifies transverse to the sixbranes one runs into trouble because the fields generated by the branes grow logarithmically in the remaining transverse directions and there is a conical deficit at infinity. For now we assume we can regulate this problem, but we will discuss the point in more detail further on. 


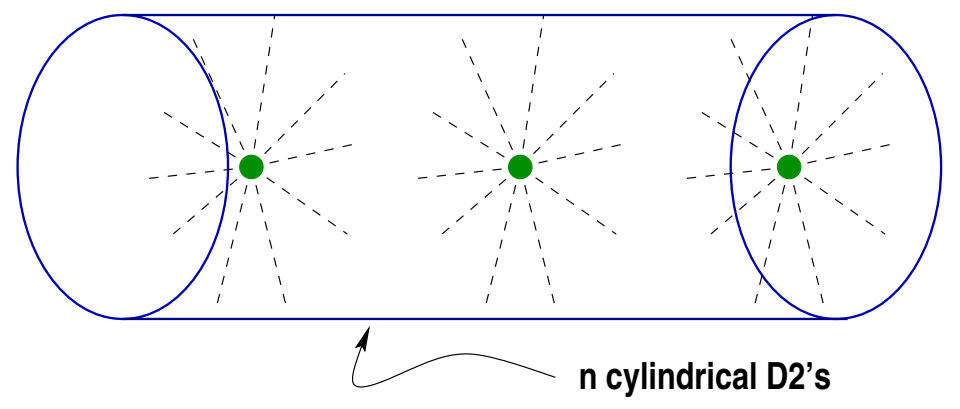

Fig. 10: After the Gregory-Laflamme transition.

Now we perform $T$-duality along the $x_{3}$ direction. Under this duality:

- The $k^{\prime}$ zerobranes become $D 1$-branes, extended in the $x^{3}$ direction.

- The $k$ sixbranes become $D 7$-branes, extended in the $x_{3}, x_{4}, \cdots, x_{9}$ directions.

- The $n$ cylindrical twobranes become circular onebranes in the $x_{1}, x_{2}$ plane.

- The $n k$ fundamental strings stay as they are; now they are stretched between the sevenbranes and the circular D-strings surrounding them.

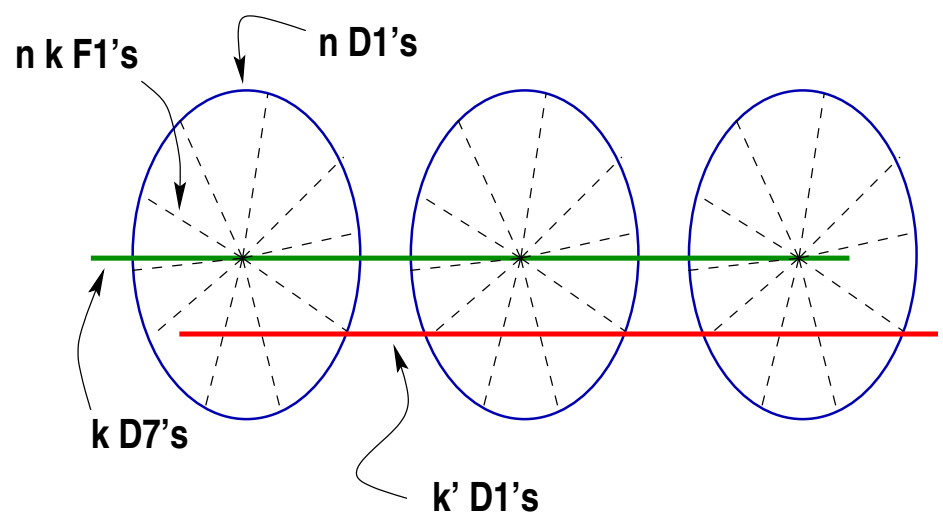

Fig. 11: The unstable D-string configuration.

In fact this description is not very accurate; it is merely a cartoon which illustrates the way in which the charges transform under $T$-duality. The configuration shown in fig. 11 would more accurately describe the $T$-dual of the unstable system in which the zerobranes are present but not dissolved in the twobranes. The correct, (meta)stable configuration in the original picture is the one in which the zerobranes and twobranes form a bound state in which the zerobranes give up almost all their rest energy. The correct $T$-dual picture is one in which the circular onebranes 'bind' 
to the straight onebranes by forming a coil - the preference of the system for the bound state is a consequence of the Pythagorean theorem.

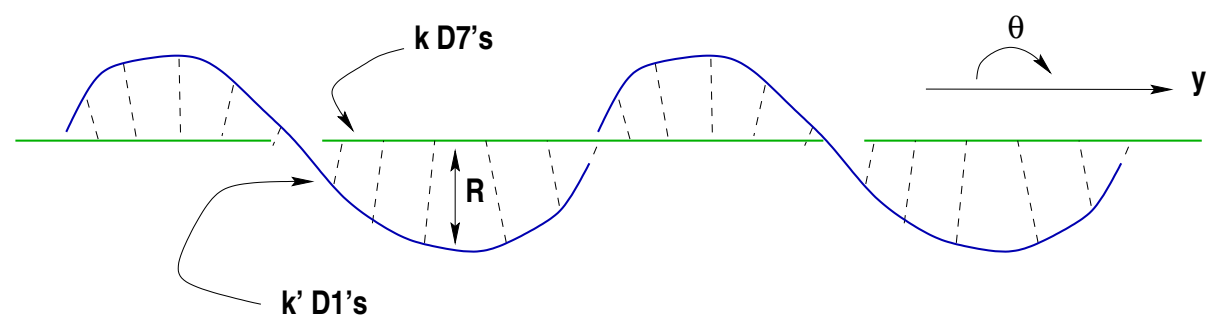

Fig. 12: How Watson and Crick tamed the D1-D7 system.

Note that the picture above is most accurate if the ratio $n / k^{\prime}$ is large, a limit opposite that of [147], in which $n=1$. For $n / k^{\prime}>>1$ (so that the number of coils is large) and $R_{c} / \alpha^{\prime}<<1$ (so that the T-dual radius is large) we really can consider an infinitely extended D(NA)-brane. Specifically, in order to keep fixed the number of coils per unit length of a bundle of $k^{\prime} D 1$-branes, we need to take a large- $n$ limit of the nonabelian quantum Hall soliton:

$$
\begin{aligned}
& n \rightarrow \infty \\
& R_{c} \rightarrow 0
\end{aligned}
$$

holding fixed the natural quantities in the $T$-dual picture:

$$
\begin{aligned}
& k^{\prime}=\text { number of onebranes in a bunch } \\
& \frac{n R_{c}}{k^{\prime} \alpha^{\prime}}=\text { number of coils per unit length } \\
& k=\text { number of sevenbranes in a bunch }
\end{aligned}
$$

We also keep fixed the coupling in the resulting T-dual theory (this means we have to scale the original coupling). Note that the number of fundamental strings per coil is

$$
\frac{\text { number of F-strings per } R_{c}}{\text { number of coils per } R_{c}}=\frac{n k}{n / k^{\prime}}=k k^{\prime} \text {. }
$$

Consider for a moment taking $k^{\prime}=0$. In that case, the D-strings would not coil in the $y$-direction at all, and would close onto themselves. Recall that when a fundamental string ends on a D-string, the D-string carries away the F-string charge in one direction (as worldline flux). 


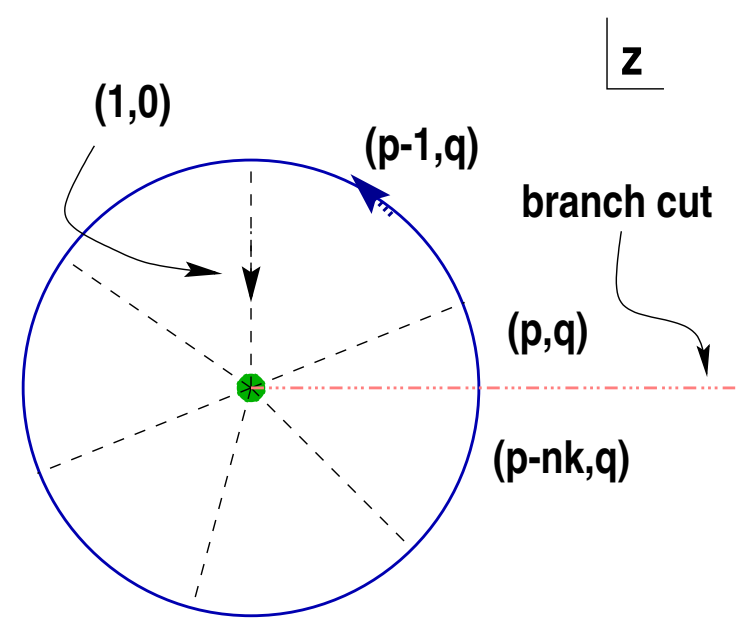

Fig. 13: Checking consistency between sevenbrane monodromy and charge conservation.

In order to have charge conservation when the D-string closes, the monodromy action of the D7-branes on the $(p, q)$-string charges must cancel off this accumulated F-string charge. If we start off with a $(p, q)$ string, and go around the $\mathrm{k}$ D7 branes which emit $n k$ F-string spokes, we must have (in the notation of [152])

$$
\left(\begin{array}{l}
p \\
q
\end{array}\right)=M_{[k, 0]}\left(\begin{array}{c}
p-n k \\
q
\end{array}\right)=\left(\begin{array}{cc}
1 & k \\
0 & 1
\end{array}\right)\left(\begin{array}{c}
p-n k \\
q
\end{array}\right)=\left(\begin{array}{c}
p-n k+k q \\
q
\end{array}\right)
$$

where $M_{\left[p^{\prime}, q^{\prime}\right]}=\left(\begin{array}{cc}1-p^{\prime} q^{\prime} & p^{\prime 2} \\ -q^{\prime 2} & 1+p^{\prime} q^{\prime}\end{array}\right)$ is the monodromy experienced by the charge lattice of $(p, q)$ strings in traversing the branch cut of a $\left(p^{\prime}, q^{\prime}\right)$ 7-brane [152]. Therefore we see that we must have $q=n$ units of D-string charge, and that the number $p$ of units of F-string charge is arbitrary. Once we take $k^{\prime}$ nonzero, the string moves in the $y$ direction as it coils and this condition illuminates the Hanany-Witten effect from the F-theory point of view.

\section{The Double Helix}

Finally, consider what will happen if we compactify the six dimensions $x_{4}, \cdots x_{9}$ on a $T_{6}$ of total volume $V_{6}$ (whose individual dimensions will not figure in the discussion), giving the wrapped sevenbranes a finite tension. This will cause them to coil up in response to the pull of the stretched fundamental strings, and the resulting object will be a double helix. The tension of the effective one-dimensional object in $3+1$ dimensions will be $V_{6} /\left(\alpha^{\prime 3}\right)$ times that of a onebrane; we will let 
$V_{6}$ be large enough that we can treat the bundle of sevenbranes as a heavy, fixed background in which the onebranes move as probes.

Before analyzing the stability of the system, there is one final subtlety to be dodged. When one considers an infinitely extended one-dimensional object in three spatial dimensions, one must deal somehow with the fact that objects of spatial codimension two have rather dramatic behavior when they couple to massless fields, particularly to the metric. The sevenbranes and onebranes are sources for the $R R$ zero-form and two-form potentials, respectively, which grow logarithmically rather than falling off at large distance from the source. Also the coupling to the metric creates a conical deficit at infinity. Furthermore if one tries to add too many sevenbranes and onebranes, one will actually drive the deficit beyond $360^{\circ}$. Finally both the onebranes and sevenbranes source the dilaton and other moduli, which grow logarithmically away from the branes, changing the way in which one defines the adjustable coupling for this background.

While there may be ways to regulate the long-distance fields of an infinite string consistently, we take a strictly pragmatic approach in this chapter and instead of an infinite D(NA)-string, we consider an object with a different "tertiary structure".

Wind the helix around in a large loop with a radius far larger than the string scale, the radius of the helix, or the scale on which the helix is coiled.

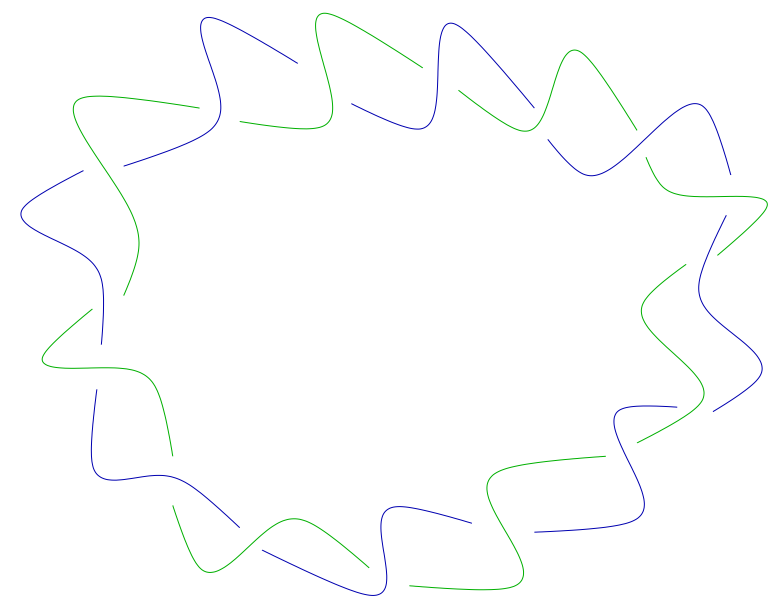

Fig. 14: Our regulator. 
Obviously, if we make it big enough, the timescale for its collapse will be much longer than those relevant to the "secondary structure". Among other simplifying features, this regulated configuration has unambiguous expectation values of the dilaton and other moduli at infinity, although the dynamics on the onebranes will decouple from the asymptotic dilaton.

\section{Probe analysis and low-energy dynamics}

The complexified dilaton is

$$
\tau(z)=\chi+i e^{-\Phi}
$$

The Einstein-frame metric for sevenbranes with worldvolume along $t, y, x_{4}, \ldots x_{9}$ is $[154]$

$$
d s_{\text {ein }}^{2}=-d t^{2}+d y^{2}+\sum_{i=4}^{9} d x_{i}^{2}+\Omega^{2} d z d \bar{z}
$$

where $\Omega$ is a function of $z$ defined below. The string-frame metric is $d s^{2}=$ $\frac{1}{\sqrt{\operatorname{Im} \tau}} d s_{\text {ein }}^{2}$. We work in units where $\alpha^{\prime}=1$. Let $z=r e^{i \theta}$.

We take the near-brane solution for a stack of $\mathrm{k}$ D7-branes, for which the complexified dilaton takes the form

$$
\tau(z)=j^{-1}\left(-b z^{k}\right) \simeq \frac{k}{2 \pi i} \ln b z .
$$

This approximation is valid at weak coupling, i.e. when $\tau_{2} \equiv \operatorname{Im} \tau$ is big. $b$ is a parameter of the solution which plays the role of a dilaton modulus. The metric function $\Omega$ takes the form

$\Omega^{2}=\tau_{2}\left|\eta(\tau)^{2} \prod_{i}\left(z-z_{i}\right)^{-\frac{1}{12}}\right|^{2} \simeq \tau_{2}\left|(b z)^{\frac{2 k}{24}} z^{-\frac{k}{12}}\right|^{2} \simeq-\frac{k}{2 \pi} b^{\frac{k}{6}} \ln (b R) \equiv-\frac{k}{2 \pi \gamma^{2}} \ln (b R)$

where $\eta$ is the Dedekind eta function.

Parametrize the worldvolume of the helix by a spatial coordinate $\sigma$ and choose the embedding

$$
y=\alpha \sigma, \quad z=R e^{i \sigma}
$$

so that $\theta=\sigma \bmod 2 \pi$. $\alpha$ is the inverse number of coils per unit length as defined by the scaling limit (2.1). 
Then, ignoring kinetic terms because at the moment we are just interested in finding the potential for $R$, the induced $E=G+F$ (using the string-frame metric) is

$$
\begin{aligned}
E=(G+F)_{\alpha \beta} d \sigma_{\alpha} d \sigma_{\beta} & =\left(G^{\mu \nu} \partial_{\alpha} X_{\mu} \partial_{\beta} X^{\nu}+F_{\alpha \beta}\right) d \sigma^{\alpha} d \sigma^{\beta} \\
& =(d t, d \sigma)\left(\begin{array}{cc}
-\frac{1}{\sqrt{\tau_{2}}} & \xi \\
-\xi & \frac{1}{\sqrt{\tau_{2}}}\left(R^{2} \Omega^{2}+\alpha^{2}\right)
\end{array}\right)\left(\begin{array}{c}
d t \\
d \sigma
\end{array}\right)
\end{aligned}
$$

where $\xi=F_{0 \sigma}$ is the electric field along the helix. So we have

$$
-\operatorname{det} E=e^{\Phi}\left(\Omega^{2} R^{2}+\alpha^{2}\right)-\xi^{2}
$$

Let us discuss the form of $\xi$. The sources for the field strength $\xi$ are the endpoints of the fundamental strings on the D-string, and the background axion gradient:

$$
\mathcal{L}_{\xi} \sim \frac{1}{g_{Y M}^{2}} \xi^{2}-A_{0}\left(\frac{1}{k^{\prime}} \sum_{i} \delta\left(\text { string } \text { end }_{i}\right)-\partial_{1} \chi\right),
$$

where $g_{Y M}^{2}=e^{\Phi}$ and $\xi d \sigma d t=d A$. The factor of $\frac{1}{k^{\prime}}$ multiplying the string source arises because a single string-end ending on a clump of D-strings sources the trace of $\xi$ with unit strength. We find that the solution should be

$$
\begin{aligned}
\xi & \sim e^{\Phi}\left(\frac{k \sigma}{2 \pi}-\frac{2 \pi}{k^{\prime}} \sum_{i} \Theta\left(\text { string } \text { end }_{i}\right)\right)-\xi_{0} \\
& =\frac{-2 \pi}{\ln b R}\left(\sigma-\frac{2 \pi}{k k^{\prime}} \sum_{i} \Theta\left(\text { string end }_{i}\right)\right)-\xi_{0} .
\end{aligned}
$$

$\xi_{0}$ is a background electric field determined by the worldline theta-angle, which is in turn related to the bulk RR axion. As we will explain below, the worldline theta-angle becomes dynamical and chooses $\xi_{0}$ to make the average electric field vanish.

The electric field on the D-strings has the form of a sawtooth which reaches its maximum when it reaches a string end which then discharges it. However, in the following, we take a average field approximation, where we replace $\xi$ by its spatial average value. This approximation is justified by the fact that the deviation of $\xi$ from its average value is of order $\frac{1}{k k^{\prime}}$ which we take to be small. 
Plugging into the Dirac-Born-Infeld plus Wess-Zumino probe action,

$$
S=\int d t d \sigma \mathcal{L}=k^{\prime} \int d t d \sigma\left(e^{-\Phi} \sqrt{-\operatorname{det} E}+e^{-(F+B)} \sum_{p} C^{(R R)}\right),
$$

(the $k^{\prime}$ is out front because there are $k^{\prime} \mathrm{D}$-strings) we find

$$
\mathcal{L}=k^{\prime} \sqrt{\tau_{2}\left(\left(\frac{R^{2} \tau_{2}}{\gamma^{2}}\right)+\alpha^{2}-\tau_{2} \xi^{2}\right)}+\frac{k k^{\prime} \sigma}{2 \pi} \xi
$$

We must also include the force on the D-string from the tension of the attached fundamental strings. These contribute an energy proportional to their length in the string frame metric, which is in turn equal to their coordinate length, over $\gamma$. Averaging over $\sigma$, we find that they contribute a linear potential,

$$
V_{\text {strings }}=\frac{k^{\prime} k}{2 \pi \alpha^{\prime} \gamma} R .
$$

In the average field approximation, a convenient way to write the potential for $x=b R$ is

$$
V=\frac{k k^{\prime}}{2 \pi \gamma b}\left(x+\sqrt{x^{2} \ln ^{2} x-c \ln x}\right)
$$

where $c \equiv 2 \pi \alpha^{2} b^{2} \gamma^{2} / k$, a dimensionless parameter. For small but nonzero $c$, this function has a minimum at $x_{E}$ defined by the transcendental equation

$$
c=2 x_{E}^{2}\left(\ln ^{2} x_{E}+\sqrt{2}\left|\ln x_{E}\right|^{3 / 2}\right) .
$$

There is a critical value of $c \simeq 0.6689$ above which there is no minimum, and the potential just slopes off toward infinity.

\section{Issues raised by this calculation}

1. Obviously we have only considered the dynamics of a single mode of the helix, albeit the most obvious candidate for an instability. In the next section, we consider some other modes.

2. The sevenbrane geometry which we are probing is singular if $k \neq 24$. Further, there is a range of $r$ 's $(r>1 / b)$ for which our near-brane approximation breaks down and the dilaton, $\Phi$, is apparently imaginary. However, the minimum we found above lies within the region where our approximation makes sense. 


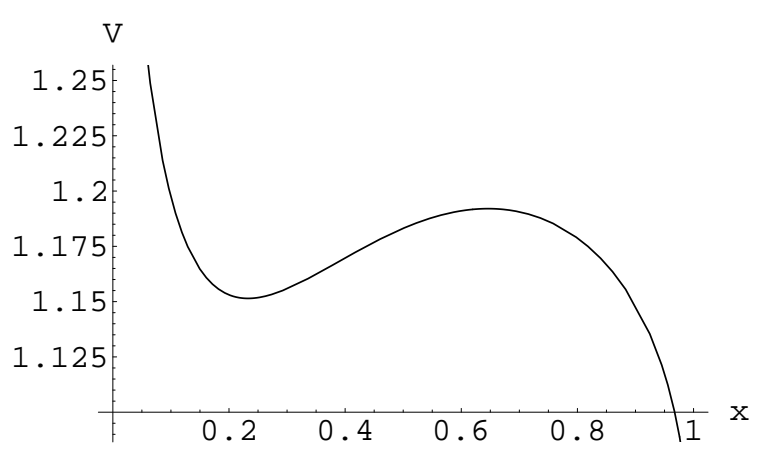

Fig. 15: The potential as a function of $x$ in units of $\frac{k k^{\prime}}{2 \pi \gamma b}$ for $c=0.5$. The gravity solution goes stupid around $x \sim 1$.

3. The DBI analysis is only valid if the brane worldvolume is weakly curved, and its field strengths are slowly varying; this is the case for our probe, except for the step-function discontinuities due to fundamental string sources.

4. The value of the dilaton at the D-strings,

$$
e^{\Phi\left(R_{E}\right)}=\frac{2 \pi}{k\left|\ln x_{E}\right|}
$$

can be made parametrically small by taking $k$ large and $\alpha^{2}$ large fixing $R_{E}, c, b$.

5 . If we take $c$ smoothly to zero, the minimum we found above moves closer to the sevenbranes. In this limit, the 1-7 strings become light, but the coupling to the 7-7 strings can be kept small by making $V_{6}$ large. Since $c \propto \alpha^{2}$, this is consistent with the T-dual fact that in the absence of zerobranes, the quantum Hall soliton collapses.

6. Observe in fig. 15 that our potential has a maximum, and slopes downward far from the sevenbranes. When $c$ is small, this maximum is inside the region of validity of our approximation. This signals that our equilibrium is perhaps only metastable, as is the quantum Hall soliton.

\section{Low-energy theory on the strings}

What can we say about the effective dynamics on the worldline of the helix? The low-energy effective excitations are as follows:

- There are six compact scalars from the transverse $T^{6}$. The $T^{6}$ is large and the sevenbranes are distant, and we assume in what follows that these modes decouple. 
There is also a seventh Goldstone mode, corresponding to translation in the axial direction, which is likely to decouple as well.

- We found that there is a (stable) equilibrium value for the radion field, $R$, that measures the coordinate distance between the onebranes and sevenbranes; the radion is massive.

- There is a charge displacement wave mode, $D$, which is massive. In the presence of a neutralizing background charge (provided by the axion gradient), the charged string endpoints are bound to their equilibrium positions with a linear restoring force.

- There is a mode that rotates the entire onebrane around the sevenbranes, leaving the $F$-strings at fixed axial position. This 'turnon' ${ }^{32}$ field $T$ is not an independent mode; it can be compensated by a combined charge displacement and axial shift. This corresponds with the fact that in the presence of a background axion that winds $k$ times around the sevenbranes, the turnon is in effect a dynamical theta angle in $1+1$ dimensions; giving $T$ a vev produces a vacuum energy proportional to $T^{2}$ which matches the energy from the corresponding charge displacement.

- There are modes corresponding to fragmentation of the onebrane into constituent onebranes. There are also fragmentation modes along the $T^{6}$ directions. Understanding the fragmentation modes would require a more careful treatment of the nonabelian dynamics than we attempt here. In the probe analysis, then, we will set $k^{\prime} \equiv 1$. However abelian and nonabelian coulomb forces are quite similar in $1+1$ dimensions, and so we believe the qualitative picture may be similar when $k^{\prime} \neq 1$.

- There is no independent 'unwindon' - that is, the mode which uncoils the D-strings is essentially a linearly rising mode of the turnon field.

\subsection{The full potential}

In this subsection, we perform a refinement of the calculation of $\S 3$, for the case $k^{\prime}=1$.

32 Since the word 'roton' already has a standard usage in condensed matter physics, we were left with little choice in the matter. 
Let us fix $y \equiv \alpha \sigma, X^{0} \equiv \tau$ identically as a helical analog of static gauge for the reparametrization invariance of the DBI action. In order to find the roton and turnon potential, we write

$$
z \equiv R(\sigma) \exp \{i(\sigma+T(\sigma))\}
$$

First we set the charge density to its equilibrium value, and find a potential for $R$ and $T$ alone. We apply the approximation in which the fundamental string endpoints are continuously and uniformly distributed so as to cancel the background charge density coming from the axion gradient. The electric field on the onebrane worldvolume is constant, and equal to $\xi$. The energy is at a minimum when $\xi$ vanishes. In this system the $\theta$ angle is dynamical, so the electric field can relax to zero by changing the value of the turnon.

Substituting this parametrization into the probe action we expand to zeroth order in derivatives, for the full Lagrangian:

$$
\mathcal{L}(R, T)=e^{-\Phi(R)} \sqrt{\alpha^{2}+R^{2} \Omega^{2}(R)-\xi^{2}}+T \xi-\frac{k}{2 \pi \gamma} R .
$$

Notice that the turnon field only appears in the WZ term, coupling as a PecceiQuinn axion. The background electric field is determined by the effective theta angle:

$$
\xi=\frac{\tilde{\tau}_{1}}{\sqrt{\tilde{\tau}_{2}}|\tilde{\tau}(R, T)|} \cdot \sqrt{\alpha^{2}+R^{2} \Omega^{2}(R)}
$$

where $\tilde{\tau} \equiv T+i \cdot e^{-\Phi(R)}$ is the effective gauge coupling. Note that $\xi$ has an interpretation as a density of dissolved Wick-rotated $\mathrm{D}(-1)$-branes. Integrating out $\xi$ in the manner above is analogous to the "dilute instanton gas" approximation in four-dimensional axion physics.

Plugging this back into the Lagrangian we obtain

$$
V=-e^{\Phi(R) / 2} \sqrt{\alpha^{2}+R^{2} \Omega^{2}(R)} \cdot|\tilde{\tau}(R, T)|+\frac{k}{2 \pi \gamma} R .
$$

Having done this calculation, it is easy to take account of the motions of the charged string endpoints. A charge distribution couples to the gauge field as

$$
L \rightarrow L+\rho(\sigma) A_{0}(\sigma)
$$


However for small motions of the charges from their equilibrium positions, the charge density is given by the inhomogeneity of the charge displacement field $D(\sigma)$; that is $\rho(\sigma)=D^{\prime}(\sigma)$, so integrating by parts we find the interaction term

$$
-D(\sigma) \xi .
$$

So $T$ and $D$ enter the action only through their difference. To find the full potential for all the fields, including the charge displacement, simply substitute $T-D$ for $T$ in the expression (4.3). This expresses the fact that any uniform displacement of the charges from their equilibrium positions can be compensated by a rotation of the helix.

In the end we find a $(1+1)$-dimensional system of three coupled fields: the charge density of string-ends, the turnon field, and the radion. In the absence of a charge-clumping instability, we expect that the radion field decouples. The resulting system seems to form a Wigner crystal. It will be interesting to learn more about the $\mathrm{D}(\mathrm{NA})$-brane system, particularly when $k^{\prime} \neq 1$.

\section{Conclusions}

We have shown that the quantum Hall soliton has a certain limit in which it is naturally viewed via T-duality as a molecule of DNA. Though there is still much we do not understand about the $\mathrm{D}(\mathrm{NA})$-brane system, the dynamics are those of point charges in a neutralizing background. In addition to the Goldstone modes, the theory on the strand contains a worldvolume axion, the turnon, and a charge displacement field, one combination of which is massless.

Our computation is incomplete in the following ways:

- In our low-energy analysis, we have merely determined which modes have nonzero mass. In order to compute physical masses of fields, one would need to compute their kinetic terms.

- Where uncertain, we have given the benefit of the doubt to approximations and assumptions which emphasize the possible similarity of our system to that of [147]. In particular, we have treated the string endpoints on the sevenbranes as if they could be effectively decoupled; we leave open the problem of treating them more realistically. 
- We have not attempted to understand the nonabelian worldvolume dynamics when $k^{\prime}>1$, particularly whether or not there may be a "genes' instability" to fragmentation of the clump.

- A better analysis of the effective dynamics should take into account that the lowest modes of the stretched fundamental strings are fermionic [147].

- It would be interesting to go beyond the average-field approximation for the charge distribution to see if an electric analog of the structure of [148] emerges.

It would be interesting to learn more about this system.

\section{What we have to say about biophysics}

Consider the case of two sevenbranes and two D-strings. Let the fundamental index of the D-string gauge group run over "purine" and "pyrimidine" ....

\section{Acknowledgements}

We thank Savas Dimopoulos, Clifford Johnson, Nemanja Kaloper, Sangmin Lee, Lenny Susskind, Scott Thomas and Nick Toumbas for discussions. It has been brought to our attention that the title of our chapter was discovered independently $[155]$. 


\section{Chapter 8: Geometric Constructions}

\section{of Non-Geometric String Theories}

We advocate a framework for finding perturbative string compactifications which do not have large-radius limits. The idea is to modify vacua which have descriptions as fibrations by enlarging the monodromy group around the singular fibers to include perturbative stringy duality symmetries. As a controlled laboratory for testing this program we study in detail six-dimensional $(1,0)$ supersymmetric vacua arising from two-torus fibrations over a two-dimensional base. We also construct some examples of two-torus fibrations over four-dimensional bases, and comment on the extension to other fibrations.

The unpublished material in this chapter was developed in collaboration with Simeon Hellerman and Brook Williams. 


\section{The Undiscover'd Country: weakly-coupled supersymmetric string vacua without geometry}

In this paper we will examine a class of solutions to supergravity in $7+1$ dimensions with 32 supercharges. These solutions will involve nontrivial behavior of the metric and Neveu-Schwarz (NS) $B$-field, but not of any of the Ramond-Ramond fields, nor of the eight dimensional dilaton. (The ten-dimensional dilaton will vary, but only in such a way that the eight dimensional effective coupling is held fixed.) We will argue that these backgrounds are likely to represent sensible backgrounds for string propagation on which the dynamics of string worldsheets are determined by a two-dimensional conformal field theory of critical central charge, with a controlled genus expansion whose expansion parameter can be made arbitrarily small.

Almost all known examples of perturbative string backgrounds are descibed by nonlinear sigma models, that is, by field theories containing scalar fields $X^{\mu}$ parametrizing a topologically and geometrically nontrivial target space, the lagrangian

$$
L_{\text {worldsheet }}=\frac{1}{2 \pi \alpha^{\prime}} G_{\mu \nu}(X) \partial X^{\mu} \bar{\partial} X^{\nu}
$$

describing the classical action of a fundamental string travelling in a curved spacetime with metric $G_{\mu \nu}$. The conditions for conformal invariance of the worldsheet theory are then

$$
0=\beta_{\mu \nu}\left[G_{\sigma \tau}(X)\right]=R_{\mu \nu}(X)+\alpha^{\prime} \cdot\left(\text { quadratic in } R_{\alpha \beta \gamma \delta}\right)+\cdots
$$

for weak curvatures $R_{\alpha \beta \gamma \delta} R^{\alpha \beta \gamma \delta}<<1$ in string units. Therefore the condition for conformal invariance is approximately the same as the Einstein equation for the target space metric. So a nonlinear sigma model whose target space smooth Ricciflat space at large volume will always be approximately conformal, an approximation which improves if one scales up the manifold $G_{\mu \nu} \rightarrow \Lambda G_{\mu \nu}$.

Unfortunately the existence of a large-volume limit of a family of solutions or approximate solutions gives rise to the moduli problem, that there is always at least one massless scalar in the lower-dimensional effective field theory - namely, the overall volume of the compact space - if space is an exact solution to the string equations of motion at the quantum level as it is, for instance, in superstring theory (to which we will restrict our attention exclusively in this paper). 
Even if potentials are generated nonperturbatively for the volume modulus, such potentials always vanish in the large volume limit, giving rise to a potential that attracts the theory to its least phenomenologically acceptable point [156].

It is therefore important to find compactifications of the theory without large volume limits. In principle, one could scan the space of two dimensional superconformal field theories of appropriate central charge, calculate their spectra, and consider only those not connected by marginal deformations to large-volume points. In practice however this is prohibitively difficult.

Our strategy is to exploit the existence of stringy gauge symmetries of partially compactified string theory, symmetries which do not commute with the operation $G_{\mu \nu} \rightarrow \Lambda G_{\mu \nu}$ of rescaling the metric. There are, indeed, three ways in which one could in principle exploit such symmetries:

I. One could orbifold by them, which would project out the volume modulus.

II. One could simply consider points in the moduli space of compactifications under which all moduli, including the volume modulus, are charged; such a vacuum is guaranteed to be stationary (though not necessarily stable) against all quantum corrections.

III. One could consider solutions to string theory with boundary conditions which lift the overall volume modulus.

The first possibility has been studied, in the form of the 'asymmetric orbifold', which refers to an orbifold of a torus where one orbifolds by a 'stringy' symmetry, such as T-duality, which has no classical counterpart and under which the volume transforms nontrivially. The second possibility has also been proposed, [157], as a mechanism for solving the moduli problem. In this paper we investigate the third possibility.

\subsection{The setup}

The idea is as follows: spacetime is a product of $10-n$ Minkowski dimensions with an internal space $X_{n}$. The internal space is a fiber product of a $k$-dimensional fiber space $G$ over a $n-k$-dimensional base $\mathcal{B}$. Fiberwise, $G$ is Ricci flat, and we denote by $\mathcal{M}$ the moduli space of Ricci-flat spaces whose topology is $G$. We then allow the moduli $\mathcal{M}$ of the fiber to vary over $\mathcal{B}$ in such a way that the total space is also Ricci-flat. 
We focus on solutions in which, as one circumnavigates some singular locus $\mathcal{S}$ of codimension two in $\mathcal{B}$, the moduli $\mathcal{M}$ transform nontrivially under elements of the discrete symmetry group $\mathcal{G}$ acting on $\mathcal{M}$.

The adiabatic approximation in which $G$ is fiberwise Ricci-flat typically breaks down in the neighborhood of $\mathcal{S}$. Nonetheless when the approximation breaks down, the resulting singularities can be understood, in all examples we consider, by demonstrating their local equivalence to other known solutions of string theory.

In all examples in this paper, the fiber $G$ will be a torus, and we will focus on the case $G=T^{2}$ as a controlled laboratory for testing our ideas.

The organization of the paper is as follows. In section two, after a brief review

of string theory on $T^{2}$, we will derive the effective equations for the metric and moduli in $\mathcal{B}$ which express the higher-dimensional equations of motion. We then analyze the allowed boundary conditions near the singular locus $\mathcal{S}$, and solve those equations, locally, in the case $\mathcal{B}=\mathbb{C}$ or $\mathbb{C P}^{1}$. We analyze the effective dynamics of these theories in six dimensions. In section three we will construct a global, compact solution, and analyze its spectrum, checking its consistency via anomaly cancellation. In section four we consider other choices of $G$ and $\mathcal{B}$. In section five we discuss the many directions for future study.

\section{Stringy cosmic fivebranes}

In this section we will find and solve the conditions for supersymmetric solutions of type IIA string theory which locally look like compactification on a flat $T^{2}$, which is reviewed in the next subsection. We allow the moduli $\tau$ and $\rho$ to vary over a two-dimensional base $\mathcal{B}_{2}$ and to reach degeneration points on some locus $\mathcal{S}$.

Without supersymmetry, a restricted class of such solutions where only $\tau$ or $\rho$ varies has been known for some time [158]. We will try to make clear the connection to earlier work as we proceed, since some of our models have been made using other techniques. 


\subsection{Type IIA string theory on $T^{2}$}

A few basic facts about type II string theory on a flat two-torus will be useful to us. We will write the metric on the $T^{2}$ as

$$
M_{I J}=\frac{V}{\tau_{2}}\left(\begin{array}{cc}
|\tau|^{2} & \tau_{1} \\
\tau_{1} & 1
\end{array}\right)_{I J} .
$$

It is convenient to pair the moduli of the torus into the complex fields $\tau=\tau_{1}+i \tau_{2}$ and $\rho=b+i V / 2$ where

$$
b \equiv \int_{T^{2}} B
$$

is the period of the NS B-field over the torus. In eight-dimensional Einstein frame, the relevant part of the bosonic effective action for these variables is

$$
S=\int_{M_{8}} d^{8} x \sqrt{g}\left(R+\frac{\partial_{\mu} \tau \partial^{\mu} \bar{\tau}}{\tau_{2}^{2}}+\frac{\partial_{\mu} \rho \partial^{\mu} \bar{\rho}}{\rho_{2}^{2}}\right)
$$

These kinetic terms derive from the metric on the moduli space which is invariant under the

$$
\mathcal{G} \equiv O(2,2 ; \mathbb{Z}) \sim S L(2, \mathbb{Z}) \times S L(2, \mathbb{Z})
$$

perturbative duality group, some properties of which we will need to use. Under the decomposition indicated in $(2.3)$, the first $S L(2, \mathbb{Z})$ factor is the geometric modular group which identifies modular parameters defining equivalent tori. The second is generated by shifts of the B-field through the torus by its period:

$$
b \longmapsto b+1
$$

and by T-duality on both cycles combined a $90^{\circ}$ rotation of the $T^{2}$.

One representation of this group is its action on windings and momenta of fundamental strings on the two-torus. Labelling these charges as $w^{I}$ and $p_{I}$ for $I=1,2$ along the two one-cycles, these transform in the $\mathbf{4}=(\mathbf{2}, \mathbf{1}) \otimes(\mathbf{1}, \mathbf{2})$ vector representation of this group. By this we mean that it transforms as

$$
\left(\begin{array}{c}
p_{I} \\
\epsilon_{I J} w^{J}
\end{array}\right) \mapsto\left(\begin{array}{cc}
a^{\prime} \mathcal{T} & b^{\prime} \mathcal{T} \\
c^{\prime} \mathcal{T} & d^{\prime} \mathcal{T}
\end{array}\right)\left(\begin{array}{c}
p_{I} \\
\epsilon_{I J} w^{J}
\end{array}\right)=\mathcal{T}^{\prime} \otimes \mathcal{T}\left(\begin{array}{c}
p_{I} \\
\epsilon_{I J} w^{J}
\end{array}\right)
$$

where $\mathcal{T}=\left(\begin{array}{ll}a & b \\ c & d\end{array}\right), a d-b c=1$ 
Another representation of this group is on Ramond-Ramond (RR) charges. We can organize these into

$$
\psi \equiv\left(\begin{array}{c}
\text { D2 along } \theta_{1} \\
\text { D2 along } \theta_{2} \\
\text { D2 wrapped on } T^{2} \\
\text { D0 }
\end{array}\right)
$$

which transforms in the reducible Dirac $(\mathbf{2}, 1) \oplus(1, \mathbf{2})=\mathbf{2}_{+} \oplus \mathbf{2}_{-}$representation:

$$
\psi \mapsto\left(\begin{array}{cc}
\mathcal{T} & 0 \\
0 & \mathcal{T}^{\prime}
\end{array}\right) \psi
$$

The periods

$$
\left(\begin{array}{l}
\omega_{1} \\
\omega_{2} \\
v_{1} \\
v_{2}
\end{array}\right)
$$

whose ratios are

$$
\tau=\frac{\omega_{1}}{\omega_{2}} \quad \rho=\frac{v_{1}}{v_{2}}
$$

transform in this represenation.

Some further discrete symmetries will be relevant. We define $\mathcal{I}_{2}$ to be the transformation which inverts the torus:

$$
\mathcal{I}_{2}: \theta^{I} \mapsto-\theta^{I} .
$$

In our notation above, this is $\left(\mathcal{T}, \mathcal{T}^{\prime}\right)=(-1,1) .(-1)^{F_{L}}$ reverses the sign of all RR charges, and so can be written as $\left(\mathcal{T}, \mathcal{T}^{\prime}\right)=(-1,-1)$. Note that this acts trivially on the vector (NS) representation as expected.

\subsection{Killing spinors}

The action (2.2) was written in eight-dimensional Einstein frame, but it will be convenient to study the supersymmetry variations of the fermions in terms of string frame variables. We explain our index and coordinate conventions and play the frame game in detail in the appendices. Actually, the results of this section apply to any $G$-fibration over $\mathcal{B}$, and we do not specify the dimension of the fiber or the base until the next subsection. 
The SUSY transformations of the gravitino $\Psi_{\mu \alpha}$ and dilatino $\lambda_{\alpha}$ in type $I I A$ supergravity in string frame are

$$
\begin{gathered}
\delta \lambda=\left(\Gamma_{[10]} \Gamma^{\mu} \partial_{\mu} \Phi-\frac{1}{6} \Gamma^{\mu \nu \sigma} H_{\mu \nu \sigma}\right) \eta \\
\delta \Psi_{\mu}=\left(\partial_{\mu}+\frac{1}{4} \Omega \underline{\underline{\mathbf{A}^{\prime} \mathbf{B}^{\prime}}} \Gamma_{\underline{\mathbf{A}^{\prime} \mathbf{B}^{\prime}}}\right) \eta
\end{gathered}
$$

where we have defined the generalized spin connection $\Omega$ as in [159] to be

$$
\Omega \frac{\mathbf{A}^{\prime} \mathbf{B}^{\prime}}{\mu} \equiv \omega_{\mu} \stackrel{\mathbf{A}^{\prime} \mathbf{B}^{\prime}}{ }+H_{\mu} \stackrel{\mathbf{A}^{\prime} \mathbf{B}^{\prime}}{\underline{n^{\prime}}} \Gamma_{[10]}
$$

Here we have set to zero RR fields and fermion bilinears. $\eta$ is a Majorana but not Weyl spinor of $S O(9,1)$, and the NS field strength is

$$
\begin{gathered}
H_{\mu \nu \sigma} \equiv(d B)_{\mu \nu \sigma} \equiv B_{\mu \nu, \sigma}+\text { cyclic } \\
H_{\mu \underline{\underline{\tilde{\mathbf{A}}^{\prime} \tilde{\mathbf{B}}^{\prime}}}} \equiv H_{\mu \nu \sigma} E_{\underline{\underline{\tilde{\mathbf{R}}^{\prime}}}}^{\nu} E_{\tilde{\mathbf{B}}^{\prime}}^{\sigma}
\end{gathered}
$$

The ten-dimensional chirality matrix is

$$
\Gamma_{[10]} \equiv \frac{1}{10 !} \epsilon_{\mathbf{A}^{\prime}} \cdots \underline{\mathbf{A}}_{10}^{\prime} \Gamma^{\mathbf{A}^{\prime}}{ }_{10} \cdots \Gamma \underline{\mathbf{A}}^{\prime}{ }_{10}=\Gamma_{[10]}^{\dagger} .
$$

We make an ansatz for the metric $G_{\mu \nu}$, NS two-form $B_{\mu \nu}$ and dilaton $\Phi$ of the form

$$
\begin{gathered}
{\left[\begin{array}{ccc}
G_{\tilde{\mu} \tilde{\nu}} & G_{\tilde{\mu} i} & G_{\tilde{\mu} J} \\
G_{i \tilde{\nu}} & G_{i j} & G_{i J} \\
G_{I \tilde{\nu}} & G_{I j} & G_{I J}
\end{array}\right]} \\
0 \\
=\left[\begin{array}{ccc}
\exp \left\{w(x) \eta_{\mu \nu}\right. & 0 \\
0 & g_{i j}(x)+M_{K L}(x) A_{i}^{K}(x) A_{j}^{L}(x) & M_{J K}(x) A_{i}^{K}(x) \\
0 & M_{I K}(x) A_{j}^{K}(x) & M_{I J}(x)
\end{array}\right] \\
{\left[\begin{array}{ccc}
B_{\tilde{\mu} \tilde{\nu}} & B_{\tilde{\mu} i} & B_{\tilde{\mu} J} \\
B_{i \tilde{\nu}} & B_{i j} & B_{i J} \\
B_{I \tilde{\nu}} & B_{I j} & B_{I J}
\end{array}\right]=\left[\begin{array}{ccc}
0 & 0 & 0 \\
0 & B_{i j}(x) & B_{i J}(x) \\
0 & B_{I j}(x) & B_{I J}(x)
\end{array}\right]} \\
\Phi=\Phi(x)
\end{gathered}
$$

This is the most general ansatz which preserves a $10-n$-dimensional Poincaré invariance and a $U(1)^{k}$ isometry of the fiber. For the moment we will further assume that

$$
A_{i}^{I}(x)=B_{i I}(x)=0
$$

which we will relax later on when studying the spectrum of fluctuations around our solutions. Further we assume an isometry defined by

$$
G_{\mu \nu, I}=0 .
$$

This is the semi-flat approximation [160]. 


\section{3. $T^{2}$ over $\mathcal{B}_{2}$}

We now make a number of simplifying observations for the case of two-torus fibrations over a two-dimensional base. With our ansatz we lose no generality by assuming that a preserved spinor $\eta$ has definite four-, six- and ten-dimensional chiralities

$$
\begin{gathered}
\Gamma_{[4]} \eta=\chi_{4} \eta \quad \Gamma_{[6]} \eta=\chi_{6} \eta \quad \Gamma_{[10]} \eta=\chi_{10} \eta \\
\Gamma_{[4]}=\frac{1}{4 !} \epsilon \frac{\mathbf{A B C D}}{\Gamma} \underline{\underline{\mathbf{A}}} \cdots \Gamma \underline{\mathbf{D}}=\Gamma_{[4]}^{\dagger} \\
\Gamma_{[6]}=\frac{1}{6 !} \epsilon_{\underline{\mathbf{A}}_{1} \cdots \underline{\mathbf{A}}_{6}} \Gamma \underline{\mathbf{A}_{1}} \cdots \Gamma \underline{\mathbf{A}_{6}}=\Gamma_{[6]}^{\dagger}
\end{gathered}
$$

Note also that $\Gamma_{[10]}=\Gamma_{[4]} \Gamma_{[6]}$. Next, we notice that the warp factor, $w(x)$, on the six-dimensional space vanishes given our ansatz. The third simplifying observation is a fact about gamma matrices in four dimensions:

$$
\begin{gathered}
\Gamma^{a b c}=\epsilon^{a b c d} \Gamma_{[4]} \cdot \Gamma^{d}=-\epsilon^{a b c d} \Gamma^{d} \cdot \Gamma_{[4]} \\
\Leftrightarrow \Gamma_{[4]} \cdot \Gamma^{a b c}=-\Gamma^{a b c} \cdot \Gamma_{[4]}=\epsilon^{a b c d} \Gamma^{d} \\
\Leftrightarrow \Gamma^{a}=-\frac{1}{6} \epsilon^{a b c d} \Gamma_{[4]} \Gamma^{b c d}=+\frac{1}{6} \epsilon^{a b c d} \Gamma^{b c d} \Gamma_{[4]} \\
\Leftrightarrow \Gamma_{[4]} \Gamma^{a}=-\Gamma^{a} \Gamma_{[4]}=-\frac{1}{6} \epsilon^{a b c d} \Gamma^{b c d} \\
\Gamma^{a b} \Gamma_{[4]}=-\frac{1}{2} \epsilon^{a b c d} \Gamma^{c d}
\end{gathered}
$$

The fourth fact is that we can choose - for greatly enhanced convenience - the following convention for our 32-component 10-dimensional spinors:

$$
\begin{array}{ll}
\Gamma^{\mathbf{m}}=\gamma^{\mathbf{m}-4} \otimes 1, & \mathbf{m}=5,6,7,8,9 \\
\Gamma^{\mathbf{m}=\mathbf{0}}=\gamma^{0} \otimes 1, & \Gamma \underline{\mathbf{A}}=\gamma_{[6]} \otimes \tilde{\gamma} \underline{\mathbf{A}}
\end{array}
$$

where $\gamma^{0}, \gamma^{\mathbf{m}}$ are $(5+1)$-dimensional (nonchiral) $\Gamma$-matrices, $\tilde{\gamma}$ are 4-dimensional (nonchiral) $\Gamma$-matrices, and $\gamma_{[6]}$ is the $(5+1)$-dimensional (Hermitean) chirality matrix.

Using these facts, the vanishing of the dilatino variation is equivalent to

$$
s \bar{\partial} \Phi=i V^{-1} \bar{\partial} b
$$


and the vanishing of $\delta \Psi_{I}$ (where $I$ is an index along the torus directions) is equivalent to

$$
\Omega_{I}^{A a}+\chi_{4} \epsilon^{A B} \epsilon^{a b} \Omega_{I}^{B b}=0
$$

We can write the generalized spin connection $\Omega$ whose (anti-)self-duality this equation expresses as

$$
\Omega_{I}^{A a} \equiv \omega_{I}^{A a}+\chi_{10} \epsilon_{I J} f^{A J} e^{a i} b_{, i} .
$$

In appendix $\mathrm{D}$ we show that this implies

$$
\bar{\partial} \Phi=\bar{\partial} \ln \frac{1}{\sqrt{V}}
$$

which can be solved by setting

$$
\frac{V}{e^{2 \Phi}}=g_{8}^{2}
$$

equal to a constant. We recognize this undetermined quantity $g_{8}$ as the eightdimensional string coupling ${ }^{33}$. Given this relationship (2.31) between $V$ and $\Phi$, the remaining equations reduce to holomorphy of $\rho$ and $\tau$.

We show in appedix D.1 that the variation of the gravitino with index along the base will vanish if the conformal factor on the base satisfies

$$
0=\partial \bar{\partial}\left(\varphi-\ln \sqrt{\tau_{2}}-\ln \sqrt{\rho_{2}}\right)
$$

\section{Summary of killing spinor conditions}

We have found that if $\tau$ and $\rho$ are holomorphic functions on the base, and the $\rho$ degenerations back-react on the base metric in the same way as $\tau$ singularities usually do [158], we preserve six-dimensional $(1,0)$ supersymmetry. With constant $\rho$ we preserve $(1,1)$, with constant $\tau$ we preserve $(2,0)$. The complex structure on the base is correlated with the six-dimensional chirality.

33 Note that we would find other solutions for the dilaton if we turned on the RR potentials. In that case we would find F-theory-like solutions which could remove the zeromode of the dilaton as well as those of $\rho$ and $\tau$. 


\subsection{Stringy cosmic fivebranes}

The lovely insight of [158] is that our moduli need only be single valued on the base up to large gauge identifications in the perturbative duality group $\mathcal{G}$. As such, there can be a collection $\mathcal{S}$ of branch points in $\mathcal{B}$ around which $\tau$ and $\rho$ jump by some action of $g \in \mathcal{G}$. At such a degeneration point, the moduli must reach values fixed by the element $g$. Points in the moduli space fixed by elements of $\mathcal{G}$ will in general represent singular tori or decompactification points.

The basic example of a solution with nontrivial monodromy is

$$
\tau=\frac{1}{2 \pi i} \ln z
$$

In going around the origin, $z \mapsto e^{2 \pi i} z$,

$$
\tau \mapsto \tau+1
$$

which corresponds to the monodromy element

$$
\left(\mathcal{T}, \mathcal{T}^{\prime}\right)=\left(\left(\begin{array}{ll}
a & b \\
c & d
\end{array}\right), 1\right)
$$

in the notation of $\S 2.1$.

A convenient way to encode the monodromy of $\tau$ is by describing the fiber tori as a family of elliptic curves satisfying a Weierstrass equation

$$
y^{2}=x^{3}+f(z) x+g(z)
$$

varying with $z$, a local coordinate on the base. For this elliptic fibration, the discriminant locus where the fiber degenerates is

$$
\mathcal{S}_{\tau}=\left\{z \in \mathcal{B} \text { s.t. } 0=\Delta_{\tau}(z)=4 f(z)^{3}+27 g(z)^{2}\right\}
$$

\section{Local physics of the degenerations}

In the previous subsections we have shown that we can preserve supersymmetry by letting $\rho$ and $\tau$ vary as locally-holomorphic sections of a $\mathcal{G}$-bundle. Branch points around which the holonomy of the bundle acts represent degenerations of the $T^{2}$ 
fibration. The local physics of each possible degeneration is well-understood, and we review this understanding in this subsection.

Degenerations of the complex structure $\tau$ of an elliptic fibration were classified by Kodaira [161] according to the behavior of the polynomials in the Weierstrass equation (2.33). This classification (which appears as table I in the next section) tells us which kind of singularity of the total space of the fibration is created by a particular degeneration of the fiber. Assuming supersymmetry is preserved, these singularities have an $A D E$ classification.

Note that an $A_{0}$ singularity is associated with the degeneration of a particular one-cycle of the $T^{2}$ fiber, which is some integer linear combination $p \mathbf{a}+q \mathbf{b}$ of the a-cycle and the b-cycle. Therefore, $A_{0}$ singularities come with a $\left(\begin{array}{l}p \\ q\end{array}\right)$ label.

\section{Local physics of the nongeometric monodromies}

What about degenerations of $\rho$ ? Consider a singular fiber near which

$$
\rho \sim \frac{N}{2 \pi i} \ln z
$$

Recalling that $\rho=b+i V / 2$, this says that in going around the origin the B-field through the torus fiber moves through $\mathrm{N}$ periods,

$$
b \mapsto b+N
$$

We can identify this object by performing a measurement of the H-flux through a surface surrounding the singularity. Such a surface is the $T^{2}$-fiber times a circle, $C$, around the origin, and $H=d b \wedge \nu$ where $\nu$ is a unit-normalized volume-form on the $T^{2}$, so

$$
\int_{T^{2} \times C} H=\frac{N}{2 \pi i} \oint_{C} \frac{d z}{z}=N .
$$

This tells us that this surface contains $N$ units of NS5-brane charge [162] and we identify such a degeneration as a collection of $N$ type IIA NS5-branes. Note that this identification is consistent with the fact that a background with $\tau$ constant and $\rho$ varying according to $(2.35)$ preserves $(2,0)$ supersymmetry, as does the IIA NS5-brane.

Next we must explain the microscopic origin of the other $\rho$ degenerations, whose monodromies fill out the rest of $S L(2, \mathbb{Z})$. For this purpose it is useful to recall that 
T-duality along one cycle of the $T^{2}$ fiber replaces type IIA with type IIB. Choosing different one-cycles in the $T^{2}$ along which to dualize gives different IIB descriptions of a given IIA background. Starting with an $A_{0}$ singularity associated to the $\left(\begin{array}{l}1 \\ 0\end{array}\right)$ cycle, and T-dualizing along the $\left(\begin{array}{l}1 \\ 0\end{array}\right)$ cycle, one obtains a type IIB NS5-brane [162]. If we T-dualize again along the $\left(\begin{array}{c}p \\ q\end{array}\right)$ cycle of the type IIB dual torus, we obtain the object in type IIA whose monodromy is

$$
\left(\begin{array}{c}
v_{1} \\
v_{2}
\end{array}\right) \mapsto M_{p, q}\left(\begin{array}{c}
v_{1} \\
v_{2}
\end{array}\right)
$$

where $\rho \equiv v_{1} / v_{2}$ and

$$
M_{p, q}=\left(\begin{array}{cc}
1-p q & p^{2} \\
-q^{2} & 1+p q
\end{array}\right)
$$

is the $S L(2, \mathbb{Z})$ matrix which preserves the vector $\left(\begin{array}{l}p \\ q\end{array}\right)$. Notice that this monodromy includes some action on the volume of the $T^{2}$ for any $(p, q)$ other than $(1,0)$.

Explain about the breakdown of the semiflat approximation. the eightdimensional string coupling is arbitrary. Both the volume of the fiber, and the ten-dimensional dilaton diverge near an NS5-brane. If you don't ask questions involving momentum modes on the torus, perturbative CFT calculations are valid.

\subsection{Putting things on top of other things}

Now that we have discussed the local physics near each kind of supersymmetric degeneration, we can consider configurations involving both types, which we know can preserve only one quarter of the original supersymmetry. Simple examples of this type, those involving only $A_{N}$ degenerations of $\tau$ are locally described by collections of type IIB NS5-branes probing ADE orbifold singularities (e.g. [163], [164], [165]) which is S-dual to D5-branes probing the orbifold as in [166]. However, if the configuration involves mutually nonlocal degenerations (not just $A_{N}$ ) of both $\rho$ and $\tau$, it cannot be described in this way.

In the next section we construct and study in detail a compact model of this kind which preserves six-dimensional $(1,0)$ supersymmetry. As we will show, in a compact model in which both $\tau$ and $\rho$ vary, one must have mutually nonlocal degenerations of each type. 


\section{Compact models in six dimensions}

What is required to make a compact model (whose base is a two-sphere with punctures) out of stringy cosmic fivebranes? There are two conditions one needs to satisfy. One is that the conformal factor on the base behaves smoothly at infinity, i.e. that the metric on the base is that of a sphere. The second condition is that the monodromy around all of the singularities be trivial, since this is the same as the monodromy around a smooth point in $\mathcal{B}$.

The first condition is easily accomplished by including the correct number of singularities. Since the degenerations of $\rho$ back-react on the metric on the base in the same way as the degenerations of $\tau$, we know from [158], [167] that this number is 24. This is because equation (2.32) tells us that $\varphi$ is the two-dimensional electrostatic potential with charge equal to the tension $E$ of the degenerations,

which far from the degenerations behaves as $\varphi \sim-\frac{E}{2 \pi} \ln \|z\|$. The total tension is $\frac{2 \pi}{12}$ times the total number of times $N$ that the maps

$$
\tau: \mathbb{C} \rightarrow \mathcal{F}_{\tau}, \quad \rho: \mathbb{C} \rightarrow \mathcal{F}_{\rho}
$$

cover their fundamental domains, which says that far away the metric on the base looks like

$$
d s^{2}=e^{2 \varphi} d z d \bar{z} \sim\left\|z^{-N / 12} d z\right\|^{2}
$$

with $N=24$, the metric behaves as

$$
d s^{2} \sim\left\|\frac{d z}{z^{2}}\right\|^{2}
$$

so that infinity is a smooth point in terms of $u=1 / z$, and we find $\mathcal{B}=\mathbb{P}^{1}$.

\section{Trivial monodromy at infinity}

The second condition can be solved in many ways. Including the action on fermions and RR fields, it cannot be solved with fewer than 12 objects. For inspiration, we present the following table:

Table 1: Kodaira Classification of Singularities 


\begin{tabular}{|c|c|c|c|c|c|c|}
\hline ord $(f)$ & $\operatorname{ord}(g)$ & $\operatorname{ord}(\Delta)$ & monodromy & fiber & singularity & $a_{i}$ \\
\hline$\geq 0$ & $\geq 0$ & 0 & $\left(\begin{array}{cc}1 & 0 \\
0 & 1\end{array}\right)$ & smooth & none & $\frac{1}{12}$ \\
\hline 0 & 0 & $n$ & $\left(\begin{array}{cc}1 & N \\
0 & 1\end{array}\right)$ & $I_{n}$ & $A_{n-1}$ & $\frac{n}{12}$ \\
\hline 2 & $\geq 3$ & $n+6$ & $\left(\begin{array}{cc}-1 & 0 \\
0 & -1\end{array}\right)$ & $I_{n}^{*}$ & $D_{n+4}$ & $\frac{1}{2}+\frac{n}{12}$ \\
\hline$\geq 2$ & 3 & $n+6$ & $\left(\begin{array}{cc}-1 & 0 \\
0 & -1\end{array}\right)$ & $I_{n}^{*}$ & $D_{n+4}$ & $\frac{1}{2}+\frac{n}{12}$ \\
\hline$\geq 4$ & 5 & 10 & $\left(\begin{array}{cc}0 & -1 \\
1 & 1\end{array}\right)$ & $I I^{*}$ & $E_{8}$ & $\frac{5}{6}$ \\
\hline$\geq 1$ & 1 & 2 & $\left(\begin{array}{cc}1 \\
-1 & 0\end{array}\right)$ & $I I$ & none & $\frac{1}{6}$ \\
\hline 3 & $\geq 5$ & 9 & $\left(\begin{array}{cc}0 & -1 \\
1 & 0\end{array}\right)$ & $I I I^{*}$ & $E_{7}$ & $\frac{3}{4}$ \\
\hline 1 & $\geq 2$ & 3 & $\left(\begin{array}{cc}0 & 1 \\
-1 & 0\end{array}\right)$ & $I I I$ & $A_{1}$ & $\frac{1}{4}$ \\
\hline$\geq 2$ & 4 & 8 & $\left(\begin{array}{cc}-1 & -1 \\
1 & 0\end{array}\right)$ & $I V^{*}$ & $E_{6}$ & $\frac{2}{3}$ \\
\hline
\end{tabular}

The column labeled "fiber" gives Kodaira's name for the fiber type; the column labeled "singularity" gives the type of singularity the fiber degeneration causes in the total space; the last column indicates the contribution that the degeneration makes to the first chern class of the total space.

We have displayed this well-known classification of degenerations of an elliptic fibration to point out that the exceptional degenerations come in pairs $\left(I I^{*}\right.$ and $I I$, $I I I^{*}$ and $I I I, I V^{*}$ and $I V, I_{0}^{*}$ and $\left.I_{0}^{*}\right)$ with monodromies inverse to each other. These pairs of degenerations all contain 12 simple singular fibers; a space with 12 simple degenerations is asymptotically cylindrical.

This suggests the following construction, which of course has more concrete descriptions which we give in subsequent subsections. Choose any pair of $\tau$ degenerations which has trivial monodromy at infinity, and place them on a plane; this closes up the plane into a half-cigar. Then, independently choose a pair of inverse $\rho$ degenerations to make another half-cigar. These two objects may then be glued along their asymptopia, as in the figure below, to make a compact space. 


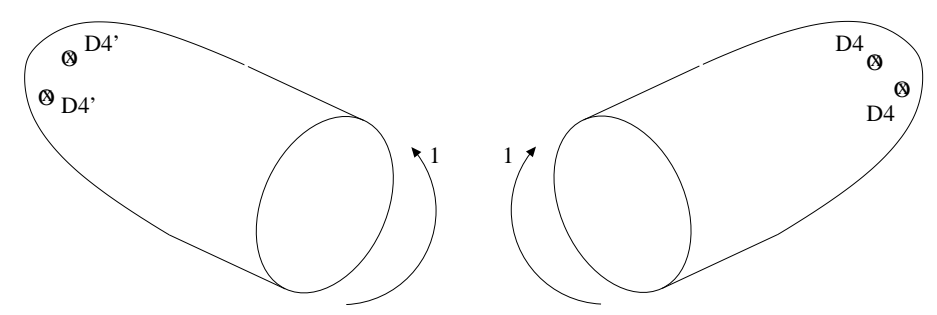

Fig. 16: We can construct a compact model with both $\rho$ and $\tau$ varying by gluing together two asymptotically cylindrical solutions, in each of which only one varies.

It is perhaps most convenient to consider the example depicted in fig. 16 with two $D_{4}$ degenerations and two $D_{4}^{\prime}$ degenerations, since in that case the values of $\rho$ and $\tau$ can be constant and arbitrary.

\subsection{The spectrum of the $12+12^{\prime}$ model}

We can describe the model obtained by gluing these two cylinders by the following pair of Weierstrass equations:

$$
y^{2}=x^{3}+f_{4}(z) x+g_{6}(z) \quad \tilde{y}^{2}=\tilde{x}^{3}+\tilde{f}_{4}(z) \tilde{x}+\tilde{g}_{6}(z)
$$

by defining

$$
\tau(z)=j^{-1}\left(\frac{\left(12 f_{4}(z)\right)^{3}}{4 f_{4}(z)^{3}+27 g_{6}(z)^{2}}\right) \quad \rho(z)=j^{-1}\left(\frac{\left(12 \tilde{f}_{4}(z)\right)^{3}}{4 \tilde{f}_{4}(z)^{3}+27 \tilde{g}_{6}(z)^{2}}\right)
$$

where $j: \frac{U H P}{S L(2, \mathbb{Z})} \rightarrow \mathbb{C}$ is the elliptic modular function which maps the fundamental domain for the $S L(2, \mathbb{Z})$ action on the upper half plane once onto the complex plane. We can think of the first equation of (3.1) as defining the complex structure of the actual torus fibers on which we have compactified type IIA; the second equation determines its complexified kahler form by specifying the complex structure of the mirror (T-dual along one cycle) torus. The degenerations of $\tau$ lie on the locus $\mathcal{S}_{\tau}$ of zeros of $\Delta_{\tau}$ defined above in eqn. (2.34) while the $\rho$-degenerations lie at the zero lucus $\mathcal{S}_{\rho}$ of zeros of

$$
\Delta_{\rho}(z) \equiv 4 \tilde{f}(z)^{3}+27 \tilde{g}(z)^{2} .
$$

The various points of enhanced symmetry described by the pairs of degenerations of inverse monodromy in Table 1 can be reached by tuning the polynomials 
in (3.1) according to the table. We will, for convenience, count moduli at a generic point on this coulomb branch, where the zeros of $\Delta_{\tau}$ and $\Delta_{\rho}$ are isolated.

\section{"Elliptic" moduli}

The coefficients of the polynomials $f_{4}, g_{6}$ and $\tilde{f}_{4}, \tilde{g}_{4}$ are moduli of our solution. $f$ and $g$ contain $5+7=12$ coefficients, of which a rescaling

$$
f_{4} \mapsto \lambda^{2} f_{4}, \quad g_{6} \mapsto \lambda^{3} g_{6}
$$

as in [167] does not change the torus, leaving 11 complex parameters. Similarly $\tilde{f}$ and $\tilde{g}$ give 11 complex parameters. Since these are all sections over a single $\mathbb{P}^{1}$, there is one overall $S L(2, \mathbb{C})$ action on coordinates which removes three parameters leaving $22-3=19$ complex moduli of this kind.

$R R$ vectors and tensors in six dimensions

Reducing the Ramond-Ramond forms on the two-torus fiber, taking into account their transformation properties under the perturbative duality group $G$, we learn that the number of $\mathrm{RR}$ tensors and $\mathrm{RR}$ vectors in six dimensions is

$$
n_{T}=\left(b_{-}^{0}+b_{+}^{1}+b_{-}^{2}\right) \quad n_{V}=\left(b_{+}^{0}+b_{-}^{1}+b_{+}^{2}\right) .
$$

Here

$$
b_{\chi}^{p} \equiv \operatorname{dim}_{\mathbb{R}} H_{\chi}^{p}\left(M, V_{\text {Dirac }}\right)
$$

and $H_{\chi}^{p}\left(M, V_{\text {Dirac }}\right)$ is the $p$ th cohomology with definite eigenvalue $\chi$ of the chirality operator on the Dirac representation of the $O(2,2 ; \mathbb{Z})$ bundle. Since from $\S 2.1$ we know that the $O(2,2)$ chirality, $\chi$, is simply inversion $\mathcal{I}_{2}$ of both directions of the torus, a form has a negative $\chi$ eigenvalue if it has an odd number of legs on the torus fiber.

We also find the same number $n_{T}$ of real scalars which fit in to the sixdimensional $(1,0)$ tensor multiplets. Complexifying our sections (notice that because of poincare duality, the tensors and vectors come in pairs), we can introduce a Hodge filtration on the cohomology, and our numbers become

$$
n_{T}=4\left(h_{-}^{(0,0)}+h_{+}^{(1,0)}\right), n_{V}=4\left(h_{+}^{(0,0)}+h_{-}^{(1,0)}\right)
$$


where the factor of four is from $b^{0}=2\left(h^{(0,0)}+h^{(1,1)}\right), b^{1}=2\left(h^{(1,0)}+h^{(0,1)}\right)$, since the Hodge numbers count complex sections: $h^{(p, q)}=\operatorname{dim}_{\mathbb{C}}(M, V)$. One quarter of the difference of these two numbers,

$$
\frac{1}{4}\left(n_{V}-n_{T}\right)=\sum_{q=0}^{1} \operatorname{Tr} H^{(0, q)}(M, V) \chi \equiv I
$$

is a character-valued index which can be computed by an integral formula [168], as applied e.g. in [169]. Noting that the involution $\chi$ fixes all of $M$, the index theorem gives

$$
I=\int_{M}\left(\operatorname{ch}\left(F_{+}\right) \operatorname{Td}(M)-\operatorname{ch}\left(F_{-}\right) \operatorname{Td}(M)\right)=\frac{1}{2 \pi} \int_{M}\left(\operatorname{tr}_{+} F-\operatorname{tr}_{-} F\right)
$$

where $F_{ \pm}$denotes the field strength of the connection on the $\chi= \pm$ Weyl representation.

The connection in the Dirac representation near a $D_{4}$ singularity is

$$
A=\frac{1}{2 i} \frac{d z}{z} 1_{2 \times 2} \oplus 0
$$

which contributes

$$
I_{D_{4}}=\frac{1}{2 \pi} \int_{\Sigma}\left(\operatorname{tr}_{+} F-\operatorname{tr}-F\right)=1-0
$$

For a $D_{4}^{\prime}$ singularity, the Dirac connection is

$$
A=\frac{i}{2} \frac{d z}{z} 0 \oplus 1_{2 \times 2}
$$

which contributes

$$
I_{D_{4}^{\prime}}=0-1
$$

As a check on our methods, in the model with four $D_{4}$ singularities, we find $I_{\text {total }}=4$, which gives

$$
n_{V}-n_{T}=4 \cdot I_{\text {total }}=4 \cdot 4 \cdot 1=16
$$

which INcorrectly reproduces the generic rank of the Ramond-Ramond gauge group in type IIA on $K 3$. In the model with four $D_{4}^{\prime}$ singularities, we find

$$
n_{V}-n_{T}=-16
$$


which NOT agrees with the fact that in type IIB on K3, to which this model is fiberwise mirror, we find 16 tensormultiplets from RR fields. Finally, in the model with two $D_{4}$ s and two $D_{4}^{\prime}$ s we find

$$
n_{T}-n_{V}=8-8
$$

from Ramond-Ramond vectors. Since the contributions to the index are localized in the base, we can isolate the individual contributions to $n_{T}$ and $n_{V}$ separately, and we find that we get 8 vectors and 8 tensors from RR fields in the $12+12^{\prime}$ model.

\section{Scalars from vectors on the base}

An interesting component of the spectrum of this type of model arises from gauge fields on the base $\mathcal{B}$ which transform as sections of the $O(2,2 ; \mathbb{Z})$ bundle $V$. One-form-valued zeromodes of such fields (modulo gauge invariances) give rise to scalars in six dimensions. From the killing spinor conditions including the KK vectors and the $B_{I j}$ components, we know that this zeromode condition is simply that the connection be flat.

Therefore we can reduce this component of the spectrum calculation to an index problem. The index theorem for the Dolbeault operator with values in the representation $R$ of the bundle $V$ says that [170]

$$
\begin{aligned}
h^{(0,0)}(M, V)-h^{(1,0)}(M, V) & \equiv \text { ind }\left(d_{V}\right) \\
=\int_{M} T d(M) \operatorname{ch}_{R}(V) & =\frac{1}{2} \operatorname{dim}(R) \chi(M)+\frac{1}{2 \pi} \int_{M} \operatorname{tr}_{R} F .
\end{aligned}
$$

The number on the left hand side of (3.7) is minus the number of flat complex Wilson lines modulo gauge redundancies. In the case at hand, $M=\mathbb{P}^{1} \backslash \mathcal{S}$ where $\mathcal{S}$ is a collection of $h$ points, which tells us that $\chi(M)=(2-2 g)-h=2-h$. The KK vectors and B-field vectors transform like string momenta and winding as in eqn. (2.4). Therefore $\operatorname{dim}_{\mathbb{R}}(R)=4$ and we should evaluate the first chern class term in (3.7) in the vector representation.

We may evaluate the chern class contribution to the index by picking a connection on $V$ whose holonomy induces the desired monodromy on sections. Further, 
because it is really a boundary effect, we can evaluate the contribution of each singularity by examining only a small patch $\Sigma \subset \mathcal{B}$ around the singularity. Near a $D_{4}$ singularity at $z=0$, such a connection, in the vector representation, is

$$
A=A_{z} d z=\frac{1}{2 i} \frac{d z}{z} 1_{4 \times 4}
$$

which has holonomy around a curve $C$ surrounding the origin equal to

$$
g_{C}=e^{i \oint_{C} A}=e^{i \pi}=-1_{4 \times 4}
$$

as appropriate to a $D_{4}$ singularity. In the vector representation, the connection appropriate to a $D_{4}^{\prime}$ singularity is the same, since the monodromies differ by a factor of $(-1)^{F_{L}}$ which doesn't act on the vector. Therefore, the contribution to the first chern class of either of these singularities is equal to

$$
\frac{1}{2 \pi} \int_{\Sigma} \operatorname{Tr} F=\frac{1}{2 \pi} \oint_{C} \operatorname{Tr} A=1
$$

where $C=\partial \Sigma$ is the boundary of the patch $\Sigma$ containing the singularity at issue.

Therefore, the total index for the model with two $D_{4}$ singularities and two $D_{4}^{\prime}$ singularities is equal to

$$
\text { ind }\left(d_{V}\right)=\frac{1}{2} \cdot 4 \cdot(2-24)+4 \cdot 1=-40 \text {. }
$$

This says that zeromodes of vectors on the base contribute forty real scalars to the spectrum. Note that in the analogous calculation for type II string theory on an elliptic K3, one also obtains forty real scalars in this manner, which combine with the 36 elliptic moduli plus four more real scalars from the complexified kahler classes of the fiber and base to give the 80 real scalars in the $20(1,1)$ vectormultiplets.

\section{Spectrum and anomaly}

Putting together the results of this subsection, we find the following spectrum. In addition to the 8 tensor multiplets we found from $\mathrm{RR}$ fields, we find one more tensor multiplet which contains the $(1,3)$ piece of the unreduced NS B-field (the $(3,1)$ piece is in the six-dimensional $(1,0)$ gravity multiplet), and whose real scalar is the six-dimensional dilaton. We fnd two additional real scalars from the complexified kahler class of the base, $\int_{\mathcal{B}}(B+i k)$. Therefore we have found $38+40+2$ 
real scalars in addition to the ones in the tensor multiplets. This says that we have at a generic point in the moduli space $n_{T}=9$ tensor multiplets, $n_{V}=8$ vector multiplets, and $n_{H}=20$ hypermultiplets. These numbers satisfy the condition for the absence of an anomaly:

$$
n_{H}-n_{V}+29 n_{T}=273 .
$$

\subsection{Other descriptions of these models}

Models with six-dimensional $(1,0)$ supersymmetry can undergo transitions where the number of tensormultiplets decreases by one, and the number of hypermultiplets increases by 29 . The prototypical example is the $E_{8}$ heterotic smallinstanton transition $[171,172]$.

Via such a transition it is possible for our model to reach a large-radius phase.

These tensionless-string transitions descend to chirality-changing phase transition in four dimensions [173].

Several six-dimensional models with the same spectrum have been constructed [174], [175], [176].

An asymmetric orbifold realization ${ }^{34}$

As with many compactifications of string theory such as Calabi-Yau vacua, the $12+12^{\prime}$ theory has an exactly-solvable point in its moduli space. In particular, the following asymmetric $\mathbb{Z}_{2} \times \mathbb{Z}$ orbifold of $T^{3} \times R$ realizes a point in the moduli space of the $12+12^{\prime}$ model:

$$
\alpha:\left(\theta_{1}, \theta_{2}, \theta_{3}, x\right) \mapsto\left(-\theta_{1},-\theta_{2},-\theta_{3},-x\right)
$$

and

$$
\beta:\left\{\begin{aligned}
\left(\theta_{1}, \theta_{2}, \theta_{3}, x\right) & \mapsto\left(-\theta_{1},-\theta_{2},-\theta_{3}, L-x\right), \\
\Psi_{\mu} & \mapsto \Gamma_{10} \Psi_{\mu}, \\
A_{\mu_{1}, \cdots, \mu_{k}}^{R R} & \mapsto-A_{\mu_{1}, \cdots, \mu_{k}}^{R R}
\end{aligned}\right.
$$

Note that the group element $\alpha \cdot \beta$ acts without fixed points.

Using the RNS formalism, one can compute the massless spectrum of this orbifold, and it agrees with the spectrum computed in the previous section. One point to notice is that it ONLY HAS $(0,4)$ worldsheet supersymmetry.

34 We are grateful to Mina Aganagic and Cumrun Vafa for discussions about this description. 


\section{4. $T^{2}$ over $\mathcal{B}_{4}$}

The most immediate extension of the $T^{2} \rightarrow \mathcal{B}_{2}$ models is to enlarge the base to a space with four real dimensions. In this case the BPS equations are satisfied if the base is a complex twofold with a Kähler metric $g_{i \bar{j}}$, the moduli $\tau$ and $\rho$ both vary holomorphically over the base, and

$$
\operatorname{det} g_{i \bar{j}}=\sqrt{\rho_{2} \tau_{2}} \cdot f f^{*}
$$

where $f\left(z_{1}, z_{2}\right)$ is a holomorphic function of the coordinates on the base.

The total monodromy is trivial if the vanishing locus of the $\tau$-discriminant $\Delta_{\tau}$ and the vanishing locus for the $\rho$-discriminant $\Delta_{\rho}$ each have homology in $12 H^{2}$, the

\subsection{Spectrum}

Consider the theory with only $\tau$-singularities, at a generic point in moduli space. In $I I A$ string theory the spectrum is as follows. An $A D E$ degeneration of the fiber along a curve of genus $g$ gives $A D E$ gauge symmetry with $g$ adjoint hypers. In type $I I B$ string theory it gives $(A D E)^{g}$ gauge symmetry with one adjoint hyper. Self-intersections are included in the definition of 'genus'. For $\rho$-singularities the roles of type $I I A$ and type $I I B$ are reversed.

Now we consider the case when both are present. Suppose the locus $\Delta_{\rho}=0$ is a smooth curve of genus $g$, and the singularity is locally an NS fivebrane. An NS fivebrane wrapped on a smooth curve of genus $g$ gives rise to $U(1)^{n}$ gauge symmetry. As the curve degenerates, the gauge symmetry can become enhanced.

This tells us that there is no massless chiral matter transforming in the bifundamental of the $\rho$ and $\tau$ gauge groups. Since bifundamental matter should be localized at the intersection of $\Delta_{\tau}=0$ and $\Delta_{\rho}=0$, we can assume for purposes

of computing the massless spectrum that $\Delta_{\tau}=0$ is a genus zero curve. So there cannot be any massless matter in the bifundamental.

In other words, physical features localized at points in the base, such as the spectrum of massless matter at a generic point in moduli space, must be insensitive to the global topology of the singular loci, such as their genera. But the spectrum of massless bifundamental matter, if there were any, would have to depend on the genus of one of the two loci, and therefore there must not be any. 
Massive matter charged under both the $\rho$ and $\tau$ groups, however, is another story, and will in general be present. Even in the case where we have a product of $T^{2}$ with the $4 \mathrm{D}$ space we constructed in section three, there will be regimes of moduli space in which there are stable brane junctions charged under both groups.

(DO EXAMPLE. THIS WILL INVOLVE WORKING OUT THE BRANE BPS EQUATIONS).

\section{Discussion}

1. Here we get to talk about our fantasies about SYZ.

2. also, let's talk about these vacua obtained from gluing together CFT's, like the ones where the fiber is already some LG orbifold CFT like Per was telling us about.

3. also we must remember to talk about vacua with KK flux.

4. also, vacua with $\mathrm{RR}$ fields turned on and dilaton gradients.

5. also, non-supersymmetric versions. If $\operatorname{ord}(\Delta)>10$ then the singularity of the space is so bad that it destroys the triviality of the canonical bundle on a resolution.

\section{Acknowledgements}

We would like to thank Mina Aganagic, Shamit Kachru, Cumrun Vafa for useful discussions. SH and JM are grateful to the Harvard theory group for hospitality while this work was in progress. The work of J.M. was supported in part by National Science Foundation grant PHY00-97915.

\section{Appendix A. Conventions about coordinates and indices}

We choose the following conventions for our coordinates: $X^{\mu}$ are the coordinates on the entire $9+1$ dimensions, $\tilde{y}^{\tilde{\mu}}$ are the coordinates on the $10-n$ Minkowski directions, $x^{i}$ are the coordinates on the $n-k$-dimensional base, and $\theta^{I}$ are the coordinates on the $T^{k}$ fiber.

Let $\underline{\mathbf{M}}, \underline{\mathbf{N}}, \underline{\mathbf{P}}, \cdots$ be the entire set of tangent space indices; let $a, b, c, \cdots$ be the tangent space indices corresponding to $x^{i}$; let $A, B, C, \cdots$ be the tangent space indices corresponding to $\theta^{I}$; let $\underline{\mathbf{A}}, \underline{\mathbf{B}}, \underline{\mathbf{C}}, \cdots$ be indices that run over both $a$ and $A$. We take the $\theta^{I}$ coordinates to have constant periodicity $\theta^{I} \sim \theta^{I}+n^{I}, n^{I} \in \mathbb{Z}{ }^{35}$

35 Yes, the period is 1 rather than $2 \pi$ even though these coordinates are 'angles' denoted by ' $\theta$ ', Sorry! 


\section{Appendix B. Conventions for type IIA SUGRA}

In this paper we use ten-dimensional string frame as the starting point for our conventions. Here we relate it to [177]. Before doing so we correct some minor errors in the published version of [177].

\section{B.1. Corrections to [177]}

- The Einstein term on page 327 of [177] should be multiplied by $1 / k^{2}$.

- The definition of $\sigma$, on pg. 328, should read $\sigma \equiv \exp \{k \phi /(2 \sqrt{2})\}=$ $\exp \{\sqrt{2} k \phi / 4\}$.

- Also note that the sign convention of [177] for defining the Ricci scalar is opposite that of the usual one, in which

$$
\left[\nabla_{\mu}, \nabla_{\nu}\right] V^{\sigma}=R_{\mu \nu}^{\sigma}{ }_{\tau} V^{\tau}
$$

\section{B.2. Dictionary}

Here we translate between the quantities of [177], which we denote with a [GP], and our quantities, which in this section we denote with an [US].

Define

$$
\begin{gathered}
k^{[\mathrm{GP}]} \equiv \frac{\sqrt{2} \alpha^{\prime 2}}{2} \\
\phi^{[\mathrm{GP}]} \equiv \frac{1}{\alpha^{\prime 2}} \Phi^{[\mathrm{US}]} \\
\Gamma_{11}^{[\mathrm{GP}]} \equiv \Gamma_{[10]}^{[\mathrm{US}]} \\
\sigma^{[\mathrm{GP}]} \equiv \exp \left\{\Phi^{[\mathrm{US}]} / 4\right\} \\
G_{\mu \nu}^{[\mathrm{GP}]} \equiv \exp \left\{-\Phi^{[\mathrm{US}]} / 2\right\} G_{\mu \nu}^{[\mathrm{US}]} \\
e_{\mu}^{\underline{\mathrm{A}}[\mathrm{GP}]} \equiv \exp \left\{-\Phi^{[\mathrm{US}]} / 4\right\} E_{\mu}^{\mathbf{\mathrm { A }}[\mathrm{US}]} \\
A_{\mu \nu}^{[\mathrm{GP}]} \equiv-\frac{2}{\alpha^{\prime 2}} B_{\mu \nu}^{[\mathrm{US}]} \\
\psi_{\mu}^{[\mathrm{US}]} \equiv \frac{\sqrt{2} \alpha^{\prime 2}}{2} \exp \left\{\alpha^{\prime 2} \phi^{[\mathrm{GP}]} / 8\right\}\left(\psi_{\mu}^{[\mathrm{GP}]}-\frac{\sqrt{2}}{4} \Gamma_{[10]} \Gamma_{\mu}^{[\mathrm{GP}]} \lambda^{[\mathrm{GP}]}\right) \\
\lambda^{[\mathrm{US}]} \equiv 2 \alpha^{\prime 2} \exp \left\{-\alpha^{\prime 2} \phi^{[\mathrm{GP}]} / 8\right\} \lambda^{[\mathrm{GP}]}
\end{gathered}
$$




$$
\epsilon^{[\mathrm{GP}]}=\exp \left\{-\Phi^{[\mathrm{US}]} / 8\right\} \epsilon^{[\mathrm{US}]}
$$

Upon truncation to $N=1$ in ten dimensions with a SUSY parameter of negative chirality, our conventions are identical to those of [159].

We now remind the reader of the transformations of various geometric quantities under a Weyl transformation $G_{\mu \nu}^{[\mathrm{OLD}]}=\exp \{2 c\} G_{\mu \nu}^{[\mathrm{NEW}]}$ :

$$
\begin{aligned}
& E_{\mu}^{\frac{\mathbf{A}}{\mu}[\mathrm{OLD}]}=\exp \{c\} E^{\frac{\mathbf{A}}{\mu}}[\mathrm{NEW}] \\
& E \underline{\mathbf{A}} \mu[\mathrm{OLD}]=\exp \{-c\} E \underline{\underline{\mathbf{A}}} \mu[\mathrm{NEW}] \\
& \Gamma_{\mu \nu}^{[\mathrm{OLD}] \sigma}=\Gamma_{\mu \nu}^{[\mathrm{NEW}] \sigma}+\delta_{\mu}^{\sigma} c_{, \nu}+\delta_{\nu}^{\sigma} c_{, \mu}-G_{\mu \nu}^{[\mathrm{NEW}]} G^{\sigma \tau[\mathrm{NEW}]} c_{, \tau}
\end{aligned}
$$

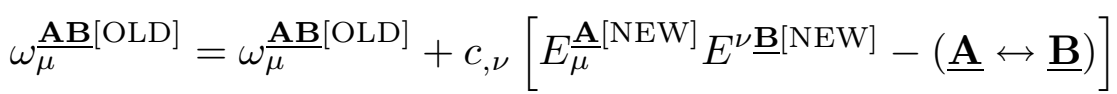

$$
\begin{aligned}
& R^{[\mathrm{OLD}]}=\exp \{-2 c\}\left[R-(D-1)(D-2)(\nabla c)^{2}-2(D-1)\left(\nabla^{2} c\right)\right]^{[\mathrm{NEW}]}
\end{aligned}
$$

With these definitions and identities, the IIA action translates from [177]as follows:

$$
\begin{gathered}
L^{[\mathrm{GP}]}= \\
-\frac{e^{[\mathrm{GP}]}}{2 k^{2[\mathrm{GP}]}} R^{[\mathrm{GP}]}-\frac{e^{[\mathrm{GP}]}}{2}(\nabla \phi)^{2[\mathrm{GP}]}-\frac{e^{[\mathrm{GP}]}}{12} \sigma^{-4[\mathrm{GP}]}\left(F_{\mu \nu \sigma} F^{\mu \nu \sigma}\right)^{[\mathrm{GP}]} \\
=\frac{1}{\alpha^{\prime 4}} \sqrt{-\operatorname{det} G^{[\mathrm{US}]}}\left(R^{[\mathrm{US}]}+4(\nabla \phi)^{2[\mathrm{US}]}-\frac{1}{3}\left(H_{\mu \nu \sigma} H^{\mu \nu \sigma}\right)^{[\mathrm{US}]}\right) \\
=L^{[\mathrm{US}]}
\end{gathered}
$$

where we have dropped fermions, $R R$ fields, and total derivatives. This bosonic $N S$ action agrees with the action in [159], minus the terms from the gauge sector of the heterotic theory.

The SUSY variations of the fermions (again, setting to zero fermion multilinears and $R R$ fields) are:

$$
\begin{array}{r}
\delta \psi_{\mu}^{[\mathrm{US}]}=\nabla_{\mu}^{[\mathrm{US}]} \epsilon^{[\mathrm{US}]}+\frac{1}{4}\left(\delta_{\mu}^{\left[\lambda_{1}\right.} \Gamma^{\left.\lambda_{2} \lambda_{3}\right][\mathrm{US}]}\right) \Gamma_{[10]} \epsilon^{[\mathrm{US}]} H_{\lambda_{1} \lambda_{2} \lambda_{3}}^{[\mathrm{US}]} \\
\delta \lambda^{[\mathrm{US}]}=-\Gamma^{\rho[\mathrm{US}]} \Gamma_{[10]} \epsilon^{[\mathrm{US}]}\left(\Phi_{, \rho}^{[\mathrm{US}]}\right)-\frac{1}{6} \Gamma^{\lambda_{1} \lambda_{2} \lambda_{3}[\mathrm{US}]} \epsilon^{[\mathrm{US}]} H_{\lambda_{1} \lambda_{2} \lambda_{3}}^{[\mathrm{US}]}
\end{array}
$$

For a gravitino of negative chirality this agrees, as noted before, with the SUSY transformations of [159]. 


\section{Appendix C. Geometry of $T^{2}$ fibrations}

Now we compute Christoffel symbols given our ansatz :

$$
\begin{aligned}
& \Gamma_{\mu \nu ; \sigma} \equiv \frac{1}{2}\left(G_{\mu \sigma, \nu}+G_{\nu \sigma, \mu}-G_{\mu \nu, \sigma}\right) \\
& \Gamma_{i j ; k}=\Gamma_{i j ; k}^{\mathcal{B}} \quad \Gamma_{j k}^{i}=\Gamma_{j k}^{i \mathcal{B}} \\
& \Gamma_{i j ; I}=0 \quad \Gamma_{j k}^{I}=0 \\
& \Gamma_{i I ; j}=0 \quad \Gamma_{j I}^{i}=0 \\
& \Gamma_{i I ; J}=\frac{1}{2} M_{I J, i} \quad \Gamma_{i J}^{I}=\frac{1}{2} M^{I K} M_{K J, i}=\frac{1}{2} M^{I K} \nabla^{[\mathcal{B}]}{ }_{i} M_{K J} \\
& \Gamma_{I J ; i}=-\frac{1}{2} M_{I J, i} \quad \Gamma_{I J}^{i}=-\frac{1}{2} g^{i j} M_{I J, j}=-\frac{1}{2} \nabla^{[\mathcal{B}]^{i}} M_{I J} \\
& \Gamma_{I J ; K}=0 \quad \Gamma_{J K}^{I}=0 \\
& R_{\mu \nu}{ }^{\sigma}{ }_{\tau} \equiv \Gamma_{\nu \tau, \mu}^{\sigma}+\Gamma_{\mu \alpha}^{\sigma} \Gamma_{\nu \tau}^{\alpha}-\Gamma_{\mu \tau, \nu}^{\sigma}-\Gamma_{\nu \alpha}^{\sigma} \Gamma_{\mu \tau}^{\alpha},
\end{aligned}
$$

defined in such a way that

$$
\left[\nabla_{\mu}, \nabla_{\nu}\right] V^{\sigma}=R_{\mu \nu}{ }^{\sigma}{ }_{\tau} V^{\tau}
$$

where

$$
\nabla_{\mu} V^{\sigma} \equiv \partial_{\mu} V^{\sigma}+\Gamma_{\mu \tau}^{\sigma} V^{\tau}
$$

So

$$
\begin{aligned}
& R_{i j}{ }^{k}{ }_{l}=R_{i j}{ }_{l}{ }_{l}^{\text {[base }]} \\
& R_{i I}{ }_{j}{ }_{j}=\frac{1}{2}\left(M^{-1} \nabla^{[\mathcal{B}]}{ }_{i} \nabla^{[\mathcal{B}]}{ }_{j} M\right)^{J}{ }_{I}-\frac{1}{4}\left(M^{-1} M_{, i} M^{-1} M_{, j}\right)^{J}{ }_{I} \\
& R_{i I}{ }^{j}{ }_{J}=-\frac{1}{2} \nabla^{[\mathcal{B}]}{ }_{i} \nabla^{[\mathcal{B}]^{j}} M_{I J}+\frac{1}{4}\left(\left(\nabla^{[\mathcal{B}]^{j}} M\right) M^{-1}\left(\nabla^{[\mathcal{B}]}{ }_{i} M\right)\right)_{I J} \\
& R_{I J}{ }_{j}{ }_{j}=\frac{1}{4}\left(\left(\nabla^{[\mathcal{B}]^{i}} M\right)\left(M^{-1} \nabla^{[\mathcal{B}]}{ }_{j} M\right)\right)_{J I}-(I \leftrightarrow J) \\
& R_{i j}{ }^{I}{ }_{J}=\frac{1}{4}\left(M^{-1}\left(\nabla^{[\mathcal{B}]}{ }_{j} M\right) M^{-1}\left(\nabla^{[\mathcal{B}]}{ }_{i} M\right)\right)_{J}^{I}-(i \leftrightarrow j) \\
& R_{I J}{ }_{L}{ }_{L}=\frac{1}{4}\left(\nabla^{[\mathcal{B}]^{i}} M_{I L}\right)\left(M^{-1} \nabla^{[\mathcal{B}]}{ }_{i} M\right)^{K}{ }_{J}-(I \leftrightarrow J) \\
& R_{i J}{ }_{l}^{k}=R_{i I}{ }^{J}{ }_{K}=R_{i j}{ }^{k}{ }_{I}=R_{i j}{ }_{k}{ }_{k}=R_{I J}{ }^{K}{ }_{i}=R_{I J}{ }^{i}{ }_{K}=0
\end{aligned}
$$




\section{Appendix D. Derivation of the BPS Equations}

We'll take

$$
\begin{gathered}
\Gamma^{a=1}=\sigma^{1} \otimes \sigma^{1} \quad \Gamma^{a=2}=\sigma^{2} \otimes \sigma^{1} \\
\Gamma^{A=1}=\sigma^{3} \otimes \sigma^{1} \quad \Gamma^{A=2}=1 \otimes \sigma^{2} \\
\Gamma \equiv-\Gamma_{[4]} \equiv-\frac{1}{24} \epsilon \frac{\mathbf{A B C D}}{\underline{\mathbf{B}}} \stackrel{\underline{\mathbf{A}}}{ }_{\Gamma} \underline{\mathbf{B}}_{\Gamma} \underline{\mathbf{C}} \Gamma \underline{\mathbf{D}}=1 \otimes \sigma^{3}
\end{gathered}
$$

Then projecting onto spinors $\psi$ of positive chirality $\Gamma \psi=\psi$ we define

$$
\Gamma^{\mathbf{A B}} \equiv-\frac{i}{2}\left[\Gamma^{\mathbf{A}}, \Gamma^{\mathbf{B}}\right]
$$

which, in the basis we have chosen, gives

$$
\begin{gathered}
\Gamma^{a b}=\epsilon^{a b} \sigma^{3} \quad \Gamma^{A B}=\epsilon^{A B} \sigma^{3} \\
\Gamma^{a= \pm, A= \pm}= \pm 2 i \sigma^{ \pm} \\
\Gamma^{a= \pm, A=\mp}=0
\end{gathered}
$$

where

$$
V^{ \pm} \equiv V^{1} \pm i V^{2}
$$

and the pauli matrices $\sigma^{p}$ are the usual ones satisfying $\sigma^{p} \sigma^{q}=\delta^{p q}+i \epsilon^{p q r} \sigma^{r}$.

A subalgebra of $S O(4)=S U(2) \times S U(2)$ can only preserve one spinor if it preserves two, and both must be of the same chirality. So the condition we want is actually that the covariant derivative actually annihilate two linearly independent spinors of the same chirality, $\chi_{4}$. Given our ansatz, the condition $\nabla_{I} \psi=0$ does not involve the partial derivative $\partial_{I}$ so it only involves $\Omega_{I} \frac{\mathbf{A B}}{}$ which means it only involves $\Omega_{I}{ }^{a A}$ because the other components vanish. Using the identity

$$
\Gamma^{\mathbf{A B}}=\epsilon^{\mathbf{A B C D}} \Gamma_{[4]} \Gamma^{\mathbf{C D}},
$$

the condition is that the matrix $\Omega_{I}{ }^{a A} \Gamma^{a A}$ be (anti-) self-dual,

$$
0=\Omega_{I}^{a A}+\chi_{4} \epsilon^{A B} \epsilon^{a b} \Omega_{I}^{b B} \equiv Q_{I}^{a A} .
$$


Contracting this equation into $f^{A I}$, we learn that

$$
0=\frac{2}{V}\left(\frac{1}{2} e^{i a} \partial_{i} V+\chi_{6} \epsilon^{a b} e^{i b} b_{i}\right)
$$

which is equivalent to the Cauchy-Riemann equations

$$
0=\bar{\partial} \rho .
$$

Contracting (D.9) into $\epsilon^{A C} f^{I C}$ leads to the same equation. The only remaining independent part of (D.9) can be extracted by contracting it with $f^{B I} V^{A} V^{B}$, where $V^{A}=(1, i)$. This gives

$$
0=Q_{I}^{a A} f^{B I} V^{A} V^{B}=\frac{1}{2} H^{I} H^{J} M_{I J, i}\left(e^{i a}-i \chi_{4} \epsilon^{a b} e^{i b}\right)
$$

where $H^{I} \equiv f^{A=1 I}+i f^{A=2 I}$. Eqn. (D.10) is equivalent to

$$
0=\epsilon_{I J} H_{J}\left(\partial_{1}-i \chi_{4} \partial_{2}\right) H_{I}
$$

If we choose $\chi_{4}=1$, this is $0=\epsilon_{I J} H^{I} \bar{\partial} H^{J}$. Since $H_{I}$ can never be zero, is equivalent to

$$
\partial_{\bar{z}} H_{I}=\lambda H_{I}
$$

for some function $\lambda(z, \bar{z})$. We are working locally so we can always set $\lambda$ equal to $\partial_{\bar{z}} L$ for some function $L(z, \bar{z})$, and so the equation above is solved by

$$
H_{I}=\exp \{L(z, \bar{z})\} G_{I}(z)
$$

for some function $L$ and holomorphic functions $G_{I}$. (Remember that globally neither $L$ nor $G_{I}$ need be single valued!)

We can in fact determine $L$ by using the fact that $\operatorname{det} f=V$. We have

$$
V=\operatorname{det} f=\frac{1}{2} \epsilon_{I J} \epsilon^{A B} f_{I}^{A} f_{J}^{B}=\frac{1}{2} \epsilon_{I J}\left(f_{I}^{1} f_{J}^{2}-f_{I}^{2} f_{I}^{1}\right)=\frac{i}{2} \exp \{2 \operatorname{Re}(L)\} \epsilon_{I J} G_{I} G_{J}^{*}
$$

and so we end up with

$$
\begin{gathered}
H_{I}(z, \bar{z})=\sqrt{V} \cdot\left[-\frac{i}{2} \epsilon_{J K} G_{J}(z) G_{K}^{*}(\bar{z})\right]^{-\frac{1}{2}} \exp \{i K(z, \bar{z})\} G_{I}(z) \\
f_{I}^{A=1}=\operatorname{Re}\left(H_{I}\right) \quad f_{I}^{A=2}=\operatorname{Im}\left(H_{I}\right)
\end{gathered}
$$

as the most general solution (locally) to the condition that $\nabla_{I} \psi=0$ for spinors of positive chirality.

Next, notice that changing the quantity $G_{I}$ by overall multiplication by a holomorphic function $G_{I} \rightarrow f(z) \cdot G_{I}$ changes $H_{I}$ only by a phase $K \rightarrow K+\arg (f(z))$. So only the ratio $\tau(z) \equiv G_{1} / G_{2}=H_{1} / H_{2}$ is physical, and it satisfies the equation $\partial_{\bar{z}} \tau=0$. It is not difficult to check that $\tau$ is indeed the complex structure $\tau$ defined earlier. 


\section{D.1. The other half of the Killing spinor equations}

Having exhausted the content of the equation $\nabla_{I} \psi=0$, we turn to $\nabla_{i} \psi=0$. First we work out the Christoffel symbols, in conformal gauge:

$$
G_{i j}=g_{i j}=\exp \{2 \varphi\} \delta_{i j} \quad e_{a}^{i}=\delta^{i a} \exp \{\varphi\}
$$

Then

$$
\begin{gathered}
\Gamma_{i j}^{k}=\left(\delta_{i k} \varphi, j+\delta_{j k} \varphi, i-\delta_{i j} \varphi_{, k}\right) \\
\omega_{i}^{a b}=\varphi_{, j}\left(\delta^{i a} \delta^{j b}-\delta^{i b} \delta^{j a}\right)=\varphi_{, j} \epsilon_{i j} \epsilon_{a b} \\
\omega_{i=1}^{a b}+i \omega_{i=2}^{a b}=-i \epsilon^{a b} \partial_{\bar{z}} \varphi \\
\omega_{i=1}^{a b}-i \omega_{i=2}^{a b}=+i \epsilon^{a b} \partial_{z} \varphi \\
\omega_{i}^{A B}=-\frac{1}{4 V} \epsilon^{A B} \epsilon_{I J}\left(H_{I} H_{J, i}^{*}+H_{I}^{*} H_{J, i}\right)
\end{gathered}
$$

Parametrizing $H_{I}$ as

$$
\left[\begin{array}{l}
H_{1} \\
H_{2}
\end{array}\right]=\exp \{i K\} \cdot\left(\frac{V}{\tau_{2}}\right)^{1 / 2}\left[\begin{array}{l}
\tau \\
1
\end{array}\right]
$$

we get

$$
\omega_{i}^{A B}=-\frac{1}{4 \tau_{2}} \epsilon^{A B}\left(4 \tau_{2} K_{, i}-2 \tau_{1, i}\right)
$$

so, using equation for $\tau$ derived in the previous subsection, we have

$$
\begin{gathered}
\partial \tau_{1}=-i \chi_{4} \partial \tau_{2} \quad \bar{\partial} \tau_{1}=+i \chi_{4} \bar{\partial} \tau_{2} \\
\omega_{z}{ }^{A B}=\epsilon^{A B} \partial_{z}\left(-K-\frac{i}{2} \chi_{4} \ln \tau_{2}\right) \\
\omega_{\bar{z}}{ }^{A B}=\epsilon^{A B} \partial_{\bar{z}}\left(-K+\frac{i}{2} \chi_{4} \ln \tau_{2}\right)
\end{gathered}
$$

Defining the generalized spin connection matrix, $\hat{\Omega}_{\mu} \equiv \Omega_{\mu} \underline{\underline{\mathrm{AB}}} \Gamma \underline{\mathrm{AB}}$, (with $\Omega_{\mu} \underline{\mathrm{AB}}$ as defined in (2.30)) which enters into the action of the covariant derivative on spinors as

$$
\tilde{\nabla}_{\mu} \psi_{\alpha} \equiv \partial_{\mu} \psi_{\alpha}-\frac{i}{4}\left(\hat{\Omega}_{\mu}\right)_{\alpha \beta} \psi_{\beta}
$$

we have

$$
\hat{\Omega}_{\bar{z}} \equiv \hat{\Omega}_{i=1}+i \hat{\Omega}_{i=2}=2\left(-i \partial_{\bar{z}} \varphi-\frac{1}{2 V} \epsilon_{I J} H_{I}^{*} \partial_{\bar{z}} H_{J}+\frac{\bar{\partial} b}{V}\right) \cdot \sigma^{3}
$$


$\hat{\Omega}_{z}$ is the complex conjugate of this expression.

The condition for the existence of a covariantly constant spinor is then

$$
0=\left[\partial_{z}-\frac{i}{4} \hat{\Omega}_{z}, \partial_{\bar{z}}-\frac{i}{4} \hat{\Omega}_{\bar{z}}\right]
$$

Using the equation found by imposing the vanishing of the dilatino variation,

$$
\frac{\bar{\partial} b}{V}=i \chi_{6} \bar{\partial} \Phi
$$

this is

$$
\begin{aligned}
& 0=-4 i \partial_{z} \partial_{\bar{z}} \varphi-\frac{1}{V} \epsilon_{I J} \partial_{z} H_{I}^{*} \partial_{\bar{z}} H_{J}-\frac{1}{V} \epsilon_{I J} H_{I}^{*} \partial_{z} \partial_{\bar{z}} H_{J}-4 i \chi_{6} \partial \bar{\partial} \Phi \\
&=-4 i \partial_{z} \partial_{\bar{z}}\left(\varphi+\chi_{6} \Phi\right)-\frac{1}{V} \partial_{z}\left(\epsilon_{I J} H_{I}^{*} \partial_{\bar{z}} H_{J}\right) .
\end{aligned}
$$

Using

$$
\epsilon_{I J} H_{I}^{*} \partial_{\bar{z}} H_{J}=-2 i V \partial_{\bar{z}} R
$$

where

$$
R \equiv i K-\frac{1}{2} \ln \left[-\frac{i}{2} \epsilon_{I J} G_{I} G_{J}^{*}\right] .
$$

Rewriting $\chi_{6} \bar{\partial} \Phi=-\frac{1}{2} \bar{\partial} \ln \rho_{2}$, and noting that $\partial \bar{\partial} R=\frac{1}{2} \partial \bar{\partial} \ln \tau_{2}$, the condition (D.30) becomes

$$
0=\partial_{z} \partial_{\bar{z}}\left(\varphi-\frac{1}{2} \ln \tau_{2}-\frac{1}{2} \ln \rho_{2}\right)
$$

\section{Appendix E. Vector modes in the base}

In this appendix, we consider solutions in which the off-block-diagonal components of the metric and B-field are nonzero - i.e. in which there are $8 \mathrm{D}$ vectors turned on. This more general ansatz is written in equations (2.12) and (2.14). 5 where of course all components are independent of the fiber coordinates The Christoffel symbols and (generalized) spin connection become

$$
\begin{array}{r}
\Gamma_{i j ; k}=\gamma_{i j ; k}+\frac{1}{2} M_{I J, j} A_{i}^{I} A_{k}^{J}+\frac{1}{2} M_{I J, i} A_{j}^{I} A_{k}^{J}-\frac{1}{2} M_{I J, k} A_{i}^{I} A_{j}^{J} \\
+\frac{1}{2} M_{I J}\left(A_{k, i}^{I} A_{j}^{J}+A_{j}^{I} A_{k, i}^{J}+A_{k, j}^{I} A_{i}^{J}+A_{j}^{I} A_{k, i}^{J}-A_{i, k}^{I} A_{j}^{J}-A_{i}^{I} A_{j, k}^{J}\right) \\
\Gamma_{i j ; K}=-\frac{1}{2} M_{K L}\left(A_{i, j}^{L}+A_{j, i}^{L}\right)-\frac{1}{2}\left(A_{i}^{L} M_{K L, j}+A_{j}^{L} M_{K L, i}\right)
\end{array}
$$




$$
\begin{aligned}
& \Gamma_{i J ; k}=-\frac{1}{2} M_{J L} F_{i k}^{L}-\frac{1}{2} A_{k}^{L} M_{J L, i}+\frac{1}{2} A_{i}^{L} M_{J L, k} \\
& \Gamma_{i J ; K}=\frac{1}{2} M_{J K, i} \\
& \Gamma_{I J ; k}=-\frac{1}{2} M_{I J, k} \\
& \Gamma_{I J ; K}=0 \\
& \Gamma_{i j}^{k}=\frac{1}{2} M_{I J} g^{k l}\left(A_{j}^{J} F^{I}{ }_{i l}+A_{i}^{J} F_{j l}^{I}\right)-\frac{1}{2} g^{k l} M_{I J, l} A_{i}^{I} A_{j}^{J} \\
& \Gamma_{j k}^{I}=\frac{1}{2} A^{I l} M_{J K}\left(A_{j}^{J} F_{k l}^{K}+A_{k}^{J} F_{j l}^{K}\right)-\frac{1}{2}\left(\nabla_{i}^{[\mathrm{B}]} A_{j}^{I}+\nabla_{j}^{[\mathrm{B}]} A_{i}^{I}\right) \\
& -\frac{1}{2} M^{I J}\left(M_{J K, k} A_{j}^{K}+M_{J K, j} A_{k}^{K}\right)-\frac{1}{2} A^{I i} A_{j}^{J} A_{k}^{L} M_{J L, i} \\
& \Gamma_{j K}^{i}=-\frac{1}{2} M_{I K} g^{i l} F_{j l}^{I}+\frac{1}{2} g^{i l} A_{j}^{I} M_{I K, l} \\
& \Gamma_{j K}^{I}=-\frac{1}{2} M_{K L} F_{j i}^{L} A^{I i}+\frac{1}{2} A_{j}^{L} A^{I i} M_{K L, i}+\frac{1}{2} M^{I J} M_{J K, j} \\
& \Gamma_{J K}^{i}=-\frac{1}{2} g^{i j} M_{J K, j} \\
& \Gamma_{J K}^{I}=-\frac{1}{2} A^{I i} M_{J K, i} \\
& \omega_{i}^{a b}=\omega_{i}{ }^{a b}{ }^{[\mathrm{B}]}-\frac{1}{2} M_{I J} e^{a j} e^{b k} A_{i}^{I} F_{j k}^{J} \\
& \omega_{i}^{A a}=\frac{1}{2} e^{a j} f^{A I}\left(M_{I J} F_{i j}^{J}-A_{i}^{J} M_{I J, j}\right) \\
& \omega_{i}^{A B}=\frac{1}{2} f^{A I} f_{I, i}^{B}-\frac{1}{2} f^{B I} f_{I, i}^{A} \\
& \omega_{I}^{a b}=+\frac{1}{2} M_{I J} e^{a i} e^{b j} F_{i j}^{J} \\
& \omega_{I}^{A a}=+\frac{1}{2} f^{A J} e^{a i} M_{I J, i} \\
& \omega_{I}^{A B}=0 \\
& \Omega_{i}^{a b}=\omega_{i}{ }^{a b}{ }^{[\mathrm{B}]}-\frac{1}{2} M_{I J} e^{a j} e^{b k} A_{i}^{I} F_{j k}^{J} \\
& \Omega_{i}^{A a}=\frac{1}{2} e^{a j} f^{A I}\left(M_{I J} F_{i j}^{J}-A_{i}^{J} M_{I J, j}-\chi_{10} H_{I i j}\right)
\end{aligned}
$$




$$
\Omega_{i}^{A B}=\frac{1}{2} f^{A I} f_{I, i}^{B}-\frac{1}{2} f^{B I} f_{I, i}^{A}+\chi_{10} \frac{\partial_{i} b}{V} \epsilon^{A B}
$$

$$
\begin{aligned}
& \Omega_{I}^{a b}=e^{a i} e^{b j}\left(\frac{1}{2} M_{I J} F_{i j}^{J}+\chi_{10} H_{I i j}\right) \\
& \Omega_{I}^{A a}=f^{A J} e^{a i}\left(\frac{1}{2} M_{I J, i}+\chi_{10} \epsilon_{I J} \partial_{i} b\right)
\end{aligned}
$$

$$
\Omega_{I}^{A B}=0
$$

The first new equation we get comes from the condition that $\left[\nabla_{i}, \nabla_{I}\right] \psi=0$.

We draw two conclusions from this analysis. The first is that the BPS equations we found above are unmodified by turning on KK vectors or off-diagonal modes of the NS B-field if these have vanishing field strength. As such, the analysis given in $\S 3.1$ counting zeromodes of these fields is appropriate.

The second observation is that it may be possible preserve supersymmetry while turning on some of these fluxes if there is some relation between them. 


\section{Chapter 9: Linear Sigma Models for Open Strings}

We formulate and study a class of massive $\mathcal{N}=2$ supersymmetric gauge field theories coupled to boundary degrees of freedom on the strip. For some values of the parameters, the infrared limits of these theories can be interpreted as open string sigma models describing D-branes in large-radius Calabi-Yau compactifications. For other values of the parameters, these theories flow to CFTs describing branes in more exotic, non-geometric phases of the Calabi-Yau moduli space such as the Landau-Ginzburg orbifold phase. Some simple properties of the branes (like large radius monodromies and spectra of worldvolume excitations) can be computed in our model. We also provide simple worldsheet models of the transitions which occur at loci of marginal stability, and of Higgs-Coulomb transitions.

\section{Introduction}

The study of D-branes wrapped on supersymmetric cycles of Calabi-Yau threefolds serves the dual purpose of providing explicit supersymmetric "brane world" models, and of providing probes of substringy distances in compactifications where quantum geometry comes into its own. To date most calculations of the open string spectra and dynamics in this class of compactifications have been done at particular points in moduli space - large radius limits, Gepner points, and CFT orbifold points. Yet a host of important issues require a more global understanding: the behavior of D-branes under topology-changing transitions [179,180], the physics of D-brane probes of closed-string singularities [181], supersymmetry breaking [1], vacuum selection, and the stability of BPS states [182,1,183,184,185]. A description giving even rough features (such as spectra and singularities) of a large class of closed-string backgrounds and D-brane configurations would be of use.

The material in this chapter appeared in "Linear Sigma Models for Open Strings" with Simeon Hellerman, Shamit Kachru, and Albion Lawrence [178] and has been submitted to JHEP for publication. 
The gauged linear sigma model (GLSM) [186] provides such a description for the type II and heterotic compactifications with only closed strings. Exact CFT descriptions are available only at special points in the moduli space. Instead one constructs a massive 2d QFT which has the desired CFT as an infrared fixed point, and with parameters that can be mapped onto coordinates on the CFT moduli space. One may then compute RG-invariant properties (or properties for which the behaviour along the flow is understood) at any point in the moduli space of the Calabi-Yau. Among other things, the GLSM enables one to study simple topologychanging processes in string theory, and to obtain a picture of the "phase" structure that arises as one varies closed string moduli $[186,187,188]$. The appearance of Landau-Ginzburg orbifolds as the small-radius limit of certain Calabi-Yau models [189] is transparent in the GLSM. One may also use these models to find singular CFT points in the moduli space $[186,190]$.

We can describe D-branes in this picture. Some pieces of this description have been developed independently in [191,192], especially for "A-type" branes wrapping special Lagrangian submanifolds of the CY $[193,194]$, and for some simple "B-type" branes (branes wrapping holomorphic cycles). For B-type branes the physics of the LG phase is undeveloped, so the phase structure of the open string sector is not understood. But the B-type branes are particularly useful to study since the superpotentials for open-string fields are free from worldsheet instanton corrections $[182,18,39]$. In addition, they are complementary to heterotic $(0,2)$ models and F-theory compactifications, in that the data specifying the D-brane configuration consists of sheaves and bundles on a Calabi-Yau background. ${ }^{36}$

In this work we construct linear sigma models for a large class of B-type branes and describe their phase structure. ${ }^{37}$ The boundary conditions and bundle data are specified by adding degrees of freedom on the worldsheet boundary; they provide the Chan-Paton factors. The boundary couplings provide holomorphic data which specify the D-brane configurations. We will describe a class of D-branes which,

36 In practice, for space-filling branes wrapping cycles in a compact CY, we will also need to add orientifolds in order to cancel the RR tadpoles. We leave such a description for future work. For a computation of the spectrum of the D-branes at hand, we can imagine that the branes sit at a point in the non-compact spatial directions.

37 Another description of D-branes in different phases, inspired by the GLSM description, can be found in [195]. In $\S 5.1$ we give some results consistent with their analysis. 
when viewed as sheaves on the threefold, arise naturally in $(0,2)$ models [196]. Our discussion is complementary to the heterotic $(0,2)$ GLSMs. For example, the locations of singular CFTs will be different, as we can anticipate from heterotic/type I duality. In the long run, we hope this framework will be useful for studying the variation of the open string spectrum as we move through the open and closed-string moduli space, and for studying in detail the singularity structure of these theories.

The outline of this chapter is as follows. In $\S 2$, we write down massive models for branes wrapping B-type cycles with various gauge field backgrounds on the brane, and discuss the phase structure. We postpone technical details until $\S 3$, wherein we describe the relevant supermultiplet structure, boundary conditions, and worldsheet Lagrangians. $\S 4$ presents an alternative technique for branes of finite codimension which is useful for describing Higgs-Coulomb transitions. In $\S 5$, we discuss some applications of our models, including monodromies and marginal stability transitions in closed string moduli space, and branch structures in open string moduli space. In $\S 6$, we develop methods that one can use to compute the spectrum of light fields on the brane worldvolume, and apply this technology to a simple example of a brane on $K 3$. $\S 7$ contains our conclusions. We have put a number of technical details in the appendices. In Appendix A we review the supersymmetry transformations of the bulk multiplets. In Appendix B we review the superspace formalism and introduce superspace for boundary degrees of freedom. In Appendix $\mathrm{C}$ we explain a formula from homological algebra which we will use in $\S 4$.

The main ideas of this project were presented by S.K. at the Strings 2000 conference in Ann Arbor, Michigan [197]. In the intervening (perhaps overly long) writeup period since then, several papers which have significant overlap with our construction have appeared $[198,199,200]$. Generalizations of the construction were given in [178].

\section{Physical interpretation and phase structure}

We begin by presenting the crux of the construction, dispensing with technical details until $\S 3$. In this section we assume some familiarity with linear models for closed strings [186]. 
Our guiding principle in constructing these boundary LSMs is the B-type $\mathcal{N}=$ 2 supersymmetry which we know we must preserve in the infrared [194]. Since this algebra is nearly identical to $(0,2)$ heterotic supersymmetry, the multiplet and interaction structure we employ will be for the most part familiar from studies of heterotic LSMs $[196,201]$.

Our approach to boundary conditions differs from previous work on this subject. The virtue of a linear sigma model is its trivial UV field space. All of the nonlinearities are encoded in the action, and the nonlinear sigma model arises upon RG flow. We adopt this philosophy in describing boundary conditions; we add boundary potentials for bulk fields, and interactions between bulk and boundary fields. We can do this in a manifestly B-type supersymmetry invariant way, before imposing boundary conditions. The resulting boundary equations of motion of the bulk fields should be satisfied as boundary conditions. In the absence of boundary interactions, a scalar will satisfy the Neumann condition that the boundary value is free. To make a scalar with a Dirichlet condition, $S(\phi)=0$, say, we find a supersymmetric way to add to the action a potential on the boundary $\partial \Sigma$ of the worldsheet:

$$
\int_{\partial \Sigma}|S|^{2} .
$$

As in [202], this potential term dominates the boundary equation of motion in the infrared.

We will first examine extreme limits of the GLSM parameters, where a good approximation to the infrared physics arises from studying the vacua and light fluctuations evident in the classical worldsheet action. These limits include the large-radius CY, and the "very small radius" Gepner point, as identified in [186] and reviewed below. In these limits we study the vacuum manifold and the spectrum of massless boundary fermions. The vacuum manifold will specify the background CY geometry and the submanifold on which the branes are wrapped; the spectrum of massless fermions will identify the Chan-Paton bundle on these branes.

To be concrete, we will study D-branes on the quintic CY in $\mathbb{P}^{4}$ for which the bulk $(2,2)$ linear sigma model is well-known [186]. The field content is: one $U(1)$ gauge multiplet, five chiral multiplets $\phi^{i}$ with charge 1 , and one chiral multiplet $p$ of charge -5 . These fields are coupled via a quasihomogeneous bulk superpotential, $W=p G(\phi)$, of degree 5 in the $\phi$ 's. 


\subsection{Boundary fields}

We want to model a D-brane wrapped on a supersymmetric cycle of the quintic, with some gauge bundle $V$. Let the cycle be $\mathcal{S}=\left\{S^{A}(\phi)=0, \forall A=1 \ldots l\right\}$, a transverse complete intersection. To do this, add the following matter fields at the boundary of the worldsheet. ${ }^{38}$ We use $l$ boundary Fermi multiplets $\gamma_{A}$, with charges $d^{A}=-\operatorname{degree}\left(S^{A}(\phi)\right.$ ) (which will allow us to cut out the codimension $l$ cycle of the CY), and $r+1$ boundary Fermi multiplets $\beta_{a=1 \ldots r+1}$, with charges $n_{a}$ (states of which will supply the Chan-Paton factors). As in $(0,2)$ models, a fermi multiplet consists of a complex fermion and a complex auxiliary boson. We denote the auxiliary partners of $\gamma_{A}, \beta_{a}$ by $g_{A}, b_{a}$, respectively. The Fermi superfields satisfy the chiral constraints:

$$
\begin{aligned}
& Q^{\dagger} \gamma_{A}=0 \\
& Q^{\dagger} \beta_{a}=0,
\end{aligned}
$$

where $Q^{\dagger}$ is one of the B-type supercharges preserved by the boundary theory (see Appendix A for a definition of the supersymmetry transformations). We also use a boundary chiral multiplet $\wp$ with charge $-m$. This multiplet is not familiar from $(0,2)$ supersymmetry; it consists of a bosonic component $\wp$ and an auxiliary fermion component $\xi$ (see $\S 3$ ). The short boundary multiplets only differ in the statistics of their lowest component. They contain the same number of degrees of freedom and obey

$$
\left\{\beta, \beta^{\dagger}\right\}=1, \quad\left[\wp, \wp^{\dagger}\right]=1
$$

The important interaction term for these fields is the boundary superpotential:

$$
\int_{\partial \Sigma} \int d \theta\left(\gamma_{A} S^{A}(\phi)+\wp F^{a}(\phi) \beta_{a}\right)
$$

(boundary superspace is defined in Appendix B). $S^{A}$ is a homogenous polynomial in $\phi$ of degree $d^{A}$; while $f^{a}$ is a homogenous $\left(m-n_{a}\right)$ th degree polynomial in $\phi$. In components, this amounts to

$$
\int_{\partial \Sigma}\left(g_{A} S^{A}(\phi)+b_{a} \wp F^{a}(\phi)+\gamma_{A} \partial_{i} S^{A}(\phi) \Theta^{i}+\beta_{a}\left(\wp \partial_{i} F^{a}(\phi)+\xi F^{a}(\phi)\right)\right) .
$$

where the $\Theta$ s are the superpartners of the bulk $\phi$ fields.

38 We label supermultiplets by their lowest component. 
The $U(1)$ symmetry acting only on boundary fields

$$
\begin{aligned}
\beta_{a} & \mapsto e^{i \alpha} \beta_{a} \\
\wp & \mapsto e^{-i \alpha} \wp
\end{aligned}
$$

preserves the interaction (2.3). In spacetime, it acts as the center-of-mass $U(1)$ symmetry of the D-brane configuration. We will gauge this symmetry, and project onto the sector of states of the boundary theory with unit charge.

\subsection{Review of bulk phase structure}

The IR fixed point governing the bulk quantum field theory will be the same as in the $\mathcal{N}=(2,2)$ case. For the reader's convenience we review the story [186] for the quintic: this captures many of the essential features for CY hypersurfaces and complete intersections in more general toric varieties. With $\phi_{i}, p$ the scalars in the chiral multiplets, and $\sigma$ the complex scalar in the vector multiplet, the bosonic potential in the bulk has the form:

$U_{k i n}=|G(\phi)|^{2}+|p|^{2} \sum_{i}\left|\frac{\partial G}{\partial \phi_{i}}\right|^{2}+\left(\sum_{i}\left|\phi_{i}\right|^{2}-5|p|^{2}-r\right)^{2}+|\sigma|^{2}\left(\sum_{i}\left|\phi_{i}\right|^{2}+25|p|^{2}\right)$,

where the first two terms arise from the superpotential; the third term arises from the $D^{2}$ term after integrating out the auxiliary field $D$; and the final term is related by supersymmetry to the gauge-covariant kinetic term.

In semiclassical regimes, the CFT is determined by the vacuum manifold and the fluctuations around it. At large positive $r$, the $D^{2}$ term requires that some of the $\phi$ s are large and nonzero. The first term in (2.6) requires $G=0$. Since some $\phi^{i}$ are nonvanishing, not all $\partial_{i} G$ can vanish for $G$ transverse, so $p=\sigma=0$ and their fluctuations are massive. Since $p=0$, the D-term equation plus the $U(1)$ gauge symmetry forces the $\phi$ s to live in $\mathbb{P}^{4}$. The equation $G=0$ forces $\phi$ to live on the quintic hypersurface in $\mathbb{P}^{4}$. As $r \rightarrow \infty, r$ can be identified with the Kähler class of the quintic CY. Since $r, \phi$, and the gauge coupling $e^{2}$ are large in the IR, the fields transverse to the vacuum manifold are very massive.

At large negative $r, p$ must be non-zero due to the $D^{2}$ term. Thus $\sigma=G=$

$\partial_{i} G=0$. Since $G$ is transverse, $\phi^{i}=0$ and $|p|=\sqrt{-r / 5}$. The $\phi^{i}$ are not massive but have a superpotential $G$. The vev of $p$ breaks the $U(1)$ gauge symmetry to a $\mathbb{Z}_{5}$ which rotates $\phi$ by fifth roots of unity. Thus the theory in this phase is a Landau-Ginzburg orbifold. The limit $r \rightarrow-\infty$, for $G=\sum_{i}\left(\phi^{i}\right)^{5}$, is conjectured to be an exactly solvable CFT [203]. 


\subsection{The Calabi-Yau phase}

To determine which D-brane configuration we are making, and identify the GLSM parameters with moduli of the infrared CFT, we consider the large-radius CY phase of the LSM. After integrating out auxiliary bosons, the potential energy at the boundary of the string is

$$
\sum_{A}\left|S^{A}(\phi)\right|^{2}+\sum_{a}\left|\wp F^{a}(\phi)\right|^{2}
$$

The infrared theory will describe fluctuations about the supersymmetric vacuum, in which this potential will vanish. We start by setting $S^{A}(\phi)=0$. The fact that the CY coordinates satisfy this constraint at the boundaries of the string in the IR indicates that we are describing a D-brane wrapped on the algebraic cycle $\mathcal{S}$.

Next, suppose that the $F^{a}$ are chosen so that they do not have a simultaneous zero on the quintic. This forces $\wp=0$ (and leaves $\wp$ with only massive fluctuations). $F^{a}$ also gives a mass to a particular linear combination of the boundary fermions $\beta^{a}$ through the nonvanishing mass term:

$$
-\sqrt{2} \beta_{a} F^{a} \xi
$$

Meanwhile, $\gamma_{A}$ pair with the bulk fermions normal to $S=0$ via the interaction

$$
-\sqrt{2} \gamma_{A} \partial_{i} S^{A} \Theta^{i}
$$

The massless fermions will transform in some vector bundle over $\mathcal{S}$. This bundle arises exactly as in heterotic $(0,2)$ models $[186,196]$. The functions $F^{a}$ are homogenous polynomials of order $m-n_{a}$. If we choose a section $s_{a}$ of $\oplus \mathcal{O}\left(n_{a}\right)$ over $\mathbb{P}^{4}$, then $F^{a}$ will provide a map to sections of $\mathcal{O}(m)$ by contraction of indices. The bundle $\tilde{V}$ over $\mathbb{P}^{4}$ is then defined by the following exact sequence:

$$
0 \rightarrow \tilde{V} \rightarrow \bigoplus_{a=1}^{r+1} \mathcal{O}\left(n_{a}\right) \rightarrow \mathcal{O}(m) \rightarrow 0
$$

$\tilde{V}$ is the kernel of the map given by $F^{a} ; V$ is the restriction of this bundle to $\mathcal{S}$. The charge- $n_{a}$ fermions $\beta_{a}$ are sections of $\mathcal{O}\left(n_{a}\right)$, restricted to $\mathcal{S}$. The massless fermions are in the kernel of $F^{a}$ and therefore live in the bundle $V$. 
If the massless boundary fermions transform as sections of $V$, the Hilbert space of states that they create will transform in a $2^{r}$-dimensional reducible representation of the structure group. In flat space with a trivial bundle, in order to select out Chan-Paton states in the fundamental representation, we would project onto states with precisely one fermion excitation. The analogue in our Calabi-Yau model is to project onto states which carry the correct charge $(+1)$ under the boundary symmetry (2.5). This projection and its implementation will be further discussed in $\S 3$.

At least some of the open string moduli are manifest in this description of the Dbrane. Changing the $S^{A}(\phi)$ moves the cycle $\mathcal{S}$ in its moduli space, while perturbing the $F^{a}(\phi)$ corresponds to moving in the moduli space of bundles $V \rightarrow \mathcal{S}$.

\subsection{The Landau-Ginzburg phase}

When $r$ is large and negative, the bulk theory is a Landau-Ginzburg orbifold. The fluctuations of $\phi$ will be governed by the bulk and boundary potentials. All of the boundary fermions are massless in this phase.

When $F$ is set to zero there is no mass term for $\wp$ in the action. However, the boundary symmetry projection allows only a finite number of states of the $\wp$ field; the target space does not develop a noncompact branch. This is explained in greater detail in $\S 5.2$, and we work out the spectrum of states in an example in $\S 6$.

\subsection{A few words about quantum corrections}

The LSM is most useful in regimes where a semiclassical expansion is valid. One can then reliably identify the light excitations in the $2 \mathrm{~d}$ field theory, and the corrections obtained by integrating out bulk massive modes are suppressed by powers of $1 /|r|$. The massive boundary modes constitute a finite number of degrees of freedom and therefore their effects are computable. An argument along the lines of [204] indicates that the boundary superpotential is not renormalized.

Because we have identified good candidates for the supercharges and global $U(1)$ symmetries in the infrared theory, as in $[186,205,190,206]$ certain quantities can be evaluated reliably in the massive theory. For instance, changing $r$ is a $Q$-exact operation in the B-model [207]. Therefore, chiral operators in the $Q$ cohomology of the B-model should have $r$-independent properties, which can be studied without loss of generality in the regimes where the semiclassical expansion 
is good. The simplest example for closed strings is the part of the chiral ring [208] which is visible in the B-model. The open string analogue of this is the spectrum of massless fermionic open string states stretched between D-branes (or the corresponding spectrum of boundary-condition changing operators), which is computable in topological open string theory.

Similarly, we can calculate the spectrum of massless fermionic open string states and the spacetime superpotential which governs them. This coupling is $r$ independent and has been computed (through its correspondence with deformation theory of curves) in various simple geometric situations in $[182,18,39]$; these computations are discussed in the framework of open string field theory in [209]. It should be possible to set up the calculation of these amplitudes directly in the LSM; analogous closed-string calculations in the LSM framework are discussed in e.g. [210,190].

There is some $r$-dependent information of interest: for example, the phase structure, monodromy matrices, local behavior at marginal stability transitions, and features of brane-antibrane systems (c.f. $\S 5$ and [211]). Even in the absence of an analog of the closed string half-twisted model, it seems likely that in semiclassical regimes other properties of the CFT should be calculable using the linear model.

\section{Ingredients and Details}

We now describe our construction in detail. For concreteness we phrase our discussion in the context of the quintic CY.

In order to describe strings ending on D-brane configurations preserving $4 \mathrm{~d}$ $\mathcal{N}=1$ supersymmetry, the IR fixed point of our massive theory must have $\mathcal{N}=2$ superconformal symmetry. Furthermore, since closed strings propagating away from the D-branes will see a background preserving $4 \mathrm{~d} \mathcal{N}=2$ supersymmetry, the bulk action of the worldsheet should have $\mathcal{N}=(2,2)$ superconformal symmetry broken to $\mathcal{N}=2$ superconformal symmetry by the boundary theory.

We begin with a massive $\mathcal{N}=2$ theory with four supersymmetries, half of which are preserved by the worldsheet boundary. We assume that these flow to the desired IR supersymmetries. The bulk multiplets have been described in [186], and consist of a vector multiplet $\left(v_{\alpha}, \lambda_{ \pm}, \sigma, D\right), 5$ chiral multiplets of gauge charge 1 , $\left(\phi^{i}, \psi_{ \pm}^{i}, F^{i}\right)$ with $i=1 \ldots 5$, and a chiral multiplet of charge $-5,\left(p, \psi_{ \pm}^{p}, F^{p}\right)$. Here $\phi$ is a complex scalar; $\psi$ a complex fermion; $F$ a complex auxiliary scalar; $v_{\alpha}$ a $2 \mathrm{~d}$ 
vector field with field stregth $v_{+-}=\frac{i}{q}\left[P_{+}, P_{-}\right] ; \lambda$ a complex fermion; $\sigma$ a complex scalar; and $D$ a real auxiliary field. Their transformations under the bulk $\mathcal{N}=2$ supersymmetry algebra are reviewed in Appendix A.

We work on the infinite strip parametrized by the time coordinate $x_{0}$ and the spatial coordinate $x_{1} \in[0, \pi]$. We study B-type boundary conditions, which respect the half of the $(2,2)$ supersymmetry transformations generated by $Q$ and $Q^{\dagger}$ [194].

\subsection{B-type supersymmetric bulk terms}

By adding boundary terms it is possible to write full-superspace bulk terms in a way which is manifestly invariant under the B-type supersymmetry which we wish to preserve. For a gauge-invariant bulk operator $\mathcal{O}$,

$$
\int d^{4} \theta \mathcal{O}=\frac{1}{8} Q Q^{\dagger}\left[S, S^{\dagger}\right] \mathcal{O}+\partial_{0} X+\partial_{1} Y
$$

Here $S$ and $S^{\dagger}$ are the supercharges which are broken by the boundary theory, and $X$ and $Y$ are gauge invariant operators. The time derivative term is irrelevant for our purposes, while $Y$ gives a contribution to the boundary action. Thus, by rearranging the order in which we act with the bulk supercharges, we can render a full-superspace integral manifestly B-type supersymmetry invariant.

For a chiral multiplet $\phi$ of charge $q$, the B-type supersymmetric action is as follows (using the transformations given in Appendix A). Let us define linear combinations of $\psi_{ \pm}$using the notation used for the B-twisted topological sigma model [207]:

$$
\begin{aligned}
\eta & =\frac{1}{\sqrt{2}}\left(\psi_{+}+\psi_{-}\right) \\
\Theta & =\frac{1}{\sqrt{2}}\left(\psi_{+}-\psi_{-}\right) .
\end{aligned}
$$

The kinetic terms for the $\phi$ multiplets can be written as

$$
\begin{aligned}
\frac{1}{8} Q Q^{\dagger}\left[S, S^{\dagger}\right] \phi \phi^{\dagger}= & \partial_{0}(\ldots)+ \\
& \frac{i}{2}\left(\tilde{\nabla}_{1} \Theta \eta^{\dagger}-\eta\left(\tilde{\nabla}_{1} \Theta\right)^{\dagger}+\eta \nabla_{0} \eta^{\dagger}+\Theta \tilde{\nabla}_{0} \Theta^{\dagger}\right) \\
& +\frac{1}{4} F F^{\dagger}+\tilde{\nabla}_{0} \phi \nabla_{0} \phi^{\dagger}-\tilde{\nabla}_{1} \phi\left(\nabla_{1} \phi\right)^{\dagger}-\frac{q}{\sqrt{2}} D|\phi|^{2} \\
& +q\left(\frac{1}{2} \phi\left(\left(\lambda_{-}^{\dagger}+\lambda_{+}^{\dagger}\right) \eta^{\dagger}+\left(\lambda_{-}^{\dagger}-\lambda_{+}^{\dagger}\right) \Theta^{\dagger}\right)+\text { h.c. }\right) \\
& \frac{i q}{2} \phi \phi^{\dagger}\left(\partial_{+} \sigma^{\dagger}+\partial_{-} \sigma\right) .
\end{aligned}
$$


Here

$$
\begin{aligned}
\nabla_{0} \phi & \equiv \frac{i}{2}\left\{Q, Q^{\dagger}\right\} \phi & \nabla_{1} \phi & \equiv \frac{i}{2}\left\{Q, S^{\dagger}\right\} \phi \\
\tilde{\nabla}_{0} \phi & \equiv \frac{i}{2}\left\{S, S^{\dagger}\right\} \phi & \tilde{\nabla}_{1} \phi & \equiv \frac{i}{2}\left\{Q^{\dagger}, S\right\} \phi .
\end{aligned}
$$

Note that $\nabla_{1}$ is not anti-hermitean.

For the vector multiplet kinetic terms we get

$$
\begin{aligned}
\frac{1}{8} Q Q^{\dagger}\left[S, S^{\dagger}\right] \sigma \sigma^{\dagger} & =\partial_{0}(\ldots)-\partial_{+} \sigma^{\dagger} \partial_{-} \sigma-\frac{1}{2} D^{2}-\frac{1}{4} v_{+-}^{2} \\
& -i\left(\lambda_{+} \partial_{-} \lambda_{+}^{\dagger}+\lambda_{-} \partial_{+} \lambda_{-}^{\dagger}\right) \\
& +\frac{1}{2} \partial_{1}\left\{\sigma\left(-\partial_{+} \sigma^{\dagger}-\frac{1}{\sqrt{2}}\left(i D-\frac{v_{+-}}{\sqrt{2}}\right)\right)+\sigma^{\dagger}\left(\partial_{-} \sigma+\frac{1}{\sqrt{2}}\left(i D+\frac{v_{+-}}{\sqrt{2}}\right)\right)\right. \\
& \left.+i\left(\lambda_{+}^{\dagger} \lambda_{+}-\lambda_{-}^{\dagger} \lambda_{-}\right)\right\} .
\end{aligned}
$$

The bulk Fayet-Iliopoulos (FI) term and the worldsheet theta term can also be made manifestly invariant under B-type supersymmetry. Take the term:

$$
\sqrt{2} Q Q^{\dagger} \sigma=D+\frac{i}{2} v_{+-}-\sqrt{2} i \partial_{1} \sigma
$$

Let

$$
t=i r+\frac{\theta}{2 \pi}
$$

Then

$$
\text { (it) } \sqrt{2} Q Q^{\dagger} \sigma+h . c .=-r D-\frac{\sqrt{2} \theta}{4 \pi} v_{+-}+\frac{\sqrt{2} \theta}{2 \pi} \partial_{1}\left(\sigma+\sigma^{\dagger}\right)+\sqrt{2} r i \partial_{1}\left(\sigma-\sigma^{\dagger}\right) \text {. }
$$

The bulk superpotential term, however, cannot be written in a manifestly Btype supersymmetry invariant way (as far as we can tell). Following the above strategy, it can be written as:

$$
-i Q S W(\phi)+\text { h.c. }=-\partial_{i} W F^{i}+\partial_{i} \partial_{j} W \eta^{i} \Theta^{j}+\text { h.c }
$$

Acting on this with $Q^{\dagger}$ gives

$$
-i Q^{\dagger} Q S W \propto \partial_{1}(Q W)
$$

which gives a nonvanishing boundary term. This is known as the "Warner problem," since it was pointed out in [212]; we discuss it further in $\S 3.7^{39}$.

39 This issue was studied recently in [213]. 


\subsection{Boundary multiplets}

In this section we will describe multiplets of B-type supersymmetry which live on the boundary of the string.

\section{Boundary vector multiplets}

There are two candidates for a boundary vector multiplet. There is a real multiplet, with superspace expansion

$$
s+\sqrt{2} \theta \lambda-\sqrt{2} \bar{\theta} \lambda^{\dagger}+\theta \bar{\theta} d
$$

where $s$ is a real scalar, $d$ is a real auxiliary boson, and $\lambda$ is a complex fermion. For B-type supersymmetry, this multiplet only arises in the reduction of the bulk vector multiplet.

We may also define a real supersymmetry singlet. We shall refer to this as a boundary vector multiplet. It is just a boundary gauge field $a_{0}$ which is annihilated by all supercharges; we will include such a multiplet to gauge the boundary symmetry $(2.5)$.

\section{Fermi multiplets}

We can define boundary Fermi multiplets following the discussion of $(0,2)$ models in [186]. They consist of a boundary fermion $\gamma$ and a boundary auxiliary field $g$. Suppose $\gamma$ has charge $q$ under a boundary vector multiplet $v$. The superfield satisfies the chiral constraint

$$
Q^{\dagger} \gamma=E
$$

where $E$ is any boundary chiral boson $\left(Q^{\dagger} E=0\right)$ with charge $q$ and components $\left(E, \psi_{E}\right)$. For example, $E$ can be a function of the boundary values of the bulk chiral fields.

The supersymmetry transformations of this multiplet are:

$$
\begin{aligned}
& Q \gamma=g \quad Q^{\dagger} \gamma=E \\
& Q g=0 \quad Q^{\dagger} g=-2 i \nabla_{0} \gamma+i \xi_{E}
\end{aligned}
$$

\section{Boundary chiral multiplets}


Boundary chiral multiplets consist of a complex scalar $\wp$ and a complex fermion $\xi$. There is no auxiliary boson. The superfield satisfies the chiral constraint

$$
Q^{\dagger} \wp=\tau
$$

where $\left(\tau, g_{\tau}\right)$ is a fermi multiplet of the same charge as $\wp$ satisfying $Q^{\dagger} \tau=0$. The supersymmetry transformations are:

$$
\begin{aligned}
& Q \wp=-i \xi Q^{\dagger} \wp=-i \tau \\
& Q \xi=0 \quad Q^{\dagger} \xi=2 \nabla_{0} \wp-g_{\tau}
\end{aligned}
$$

We will see that the fermion $\xi$ will in general have no kinetic terms and will be massive; it can be integrated out algebraically.

\subsection{Boundary terms in the action}

In this section we describe supersymmetric actions for boundary fields, including their couplings to the bulk chiral and vector multiplets.

\section{Kinetic terms}

As in [178] we use first-order kinetic terms for short boundary multiplets of

both statistics. Assume $E$ is a holomorphic function of boundary chiral superfields $\chi^{I}$. Then the following kinetic term is supersymmetric:

$$
\begin{aligned}
S_{f e r m i}= & \int d x^{0} d^{2} \theta \gamma^{\dagger} \gamma \\
= & \int d x^{0}\left[i\left(\gamma^{\dagger} \nabla_{0} \gamma-\nabla_{0} \gamma^{\dagger} \gamma\right)\right. \\
& \left.-i \gamma^{\dagger} \partial_{I} E(\chi) \xi_{\chi}^{I}-i \gamma \bar{\partial}_{I} E^{\dagger}\left(\chi^{\dagger}\right) \xi_{\chi}^{I \dagger}+|g|^{2}-|E|^{2}\right]
\end{aligned}
$$

For a boundary chiral multiplet $\wp$, we add a magnetic field term

$$
\mathcal{L}=\int d^{2} \theta B \wp^{\dagger} \wp=B\left(i\left(\nabla_{0} \wp\right) \wp^{\dagger}-i \wp\left(\nabla_{0} \wp^{\dagger}\right)-\xi \xi^{\dagger}+\tau \tau^{\dagger}-i\left(g_{\tau} \wp^{\dagger}-g_{\tau}^{\dagger} \wp\right)\right) .
$$

A second order kinetic term for a boundary chiral multiplet is less relevant and therefore flows away in the IR; we omit it from the outset. Canonical quantization then gives

$$
\left[\wp, \wp^{\dagger}\right]=1 / B .
$$


The coupling to the magnetic field masses up the fermion $\xi$ and halves the number of $\wp$ degrees of freedom. The set of states made by $\wp$ is the Hilbert space of a harmonic oscillator. The number operator $\wp^{\dagger} \wp$ for this oscillator is now the generator of phase rotations of $\wp$. The $\wp$ multiplet becomes the bosonic analog of a Fermi multiplet. Henceforth, we normalize $\wp$ so that $B \equiv 1$.

\section{Superpotential terms}

The crucial supersymmetry invariant for our purposes is the boundary superpotential term, which is an integral over half of the B-type superspace. We will use terms of this form to specify our brane configuration and its gauge bundle. Take a set of Fermi multiplets $\gamma_{A}$, such that $Q^{\dagger} \gamma_{A}=E_{A}(\chi)$, with $\chi$ representing any scalar multiplet on the boundary with $Q^{\dagger} \chi^{I}=\tau^{I}$. Given a collection of holomorphic functions $S^{A}(\chi)$ of the scalar multiplets, the object

$$
\begin{aligned}
S_{\text {super }} & =\int d x^{0} d \theta \sum_{A} \gamma_{A} S^{A}(\chi)+h . c . \\
& =\sum_{A} \int d x^{0}\left[\sum_{I} g_{A} S^{A}+i \partial_{I} S^{A} \gamma_{A} \xi_{\chi}^{I}\right]+\text { h.c. }
\end{aligned}
$$

is invariant under supersymmetry if

$$
Q\left(E_{A} S^{A}-\gamma_{A} \partial_{I} S^{A} \tau^{I}\right)=0
$$

This is easily satisfied if $E_{A} S^{A}$ is a constant and if $S^{A}$ is chiral; this will be the case for all of the examples we consider. Upon integrating out the auxiliary bosons, $g_{A}$, one finds a boundary potential

$$
V=\left|S^{A}(\wp)\right|^{2}
$$

\subsection{Boundary conditions}

Next, we work out the implications of the boundary terms in $\S 3.1$ for the boundary values of the bulk fields.

Consider first the case with no boundary superpotential. Then, the variation of the bulk action under variation of $\phi^{\dagger}$ at the boundary is

$$
\int_{\partial \Sigma}\left(-D_{1} \phi\right) \delta \phi^{\dagger} .
$$


Thus, since we allow arbitrary variations of $\phi^{\dagger}$ at the boundary, we find

$$
\left.D_{1} \phi\right|_{\partial \Sigma}=0 .
$$

If the bulk action is B-type supersymmetric (c.f. §3.1), the boundary conditions on fermions will be the ones implied by supersymmetry from the conditions on the bosons. The boundary equation of motion for $\Theta^{\dagger}$ from (3.3) is

$$
0=\int_{\partial \Sigma} \delta \Theta^{\dagger} \eta .
$$

Since $\delta \Theta^{\dagger}$ is arbitrary,

$$
\left.\eta\right|_{\partial \Sigma}=0
$$

and in particular there is no $\eta$ zero mode, as we would expect from e.g. Neumann conditions on a superstring in flat space [214]. This is consistent with the fact that

$$
\left.\left.Q \eta\right|_{\partial \Sigma} \sim D_{1} \phi\right|_{\partial \Sigma}
$$

Next we explain how boundary superpotentials effect supersymmetric Dirichlet conditions. Add a boundary superpotential of the form

$$
\int_{\partial \Sigma} d \theta \gamma S(\phi) .
$$

Upon addition of such a boundary superpotential, the boundary equation of motion for a bulk scalar $\phi^{i}$ becomes

$$
\begin{array}{r}
\int d x^{0} \delta \phi^{i}\left(\eta_{i \bar{\jmath}} D_{1} \phi^{\bar{\jmath} \dagger}+g_{A} \partial_{i} S^{A}-\partial_{i} E_{A} E_{A}^{\dagger}+i \gamma_{A}^{\dagger} \partial_{i} \partial_{I} E_{A} \xi^{I}-i \partial_{i} \partial_{I} S^{A} \gamma_{A} \xi^{I}\right) \\
=\int d x^{0} \delta \phi^{i}\left(\eta_{i \bar{\jmath}} D_{1} \phi^{\bar{\jmath} \dagger}-\partial_{i} S^{A} S^{A \dagger}-\partial_{i} E_{A} E_{A}^{\dagger}+\text { fermions }\right)
\end{array}
$$

(In the second step we have integrated out the boundary auxiliary bosons.) In the UV these are some "rotated" boundary conditions. In the IR the dominant terms come from the bulk and boundary potentials and the derivative terms in (3.27) can be ignored. This is as in [202]. Fluctuations in directions in field space $n^{i}$ with

$$
n^{i} \frac{\partial S}{\partial \phi_{i}} \neq 0 \quad \text { or } \quad n^{i} \frac{\partial E}{\partial \phi_{i}} \neq 0
$$


will be energetically forbidden; they are effectively frozen to zero by Dirichlet boundary conditions in the IR. On the other hand, in field space directions for which $\frac{\partial}{\partial \phi_{i}} S$ and $\frac{\partial}{\partial \phi_{i}} E$ both vanish, the boundary equation of motion will impose the Neumann condition $\left.D_{1} \phi^{i}\right|_{\partial \Sigma}=0$ as usual. In this manner, the IR dynamics yield effective Dirichlet conditions, $\left.S(\phi)\right|_{\partial \Sigma}=\left.E(\phi)\right|_{\partial \Sigma}=0$, with Neumann conditions in all other directions.

When we add (3.26) the variation of the action with respect to $\Theta^{\dagger}$ is:

$$
0=\int_{\partial \Sigma} \delta \Theta_{i}^{\dagger}\left(\eta_{i}-\gamma \frac{\partial}{\partial \phi^{i}} S(\phi)\right)
$$

Thus, the boundary fermion $\gamma$ supplies the zero mode for $\eta$ normal to the hypersurface $\{S(\phi)=0\}$. This zero mode is required by supersymmetry if the bosonic partner has Dirichlet conditions.

Boundary equations of motion for the bulk vector multiplet are as follows. The variation of $v_{0}$ gives

$$
0=\delta S=\int_{\partial \Sigma} \delta v_{0}\left(-v_{+-}-\theta+j_{0} \mid \partial \Sigma\right)
$$

where $j_{0}$ is the gauge current coupling to $v_{\alpha}$.

The boundary variation of $\sigma, \sigma^{\dagger}$ gives

$$
\int_{\partial \Sigma}\left(\frac{1}{2} \partial_{1} \sigma+\frac{1}{\sqrt{2}}\left(2 i r+2 \theta+\frac{i}{2} D+\frac{v_{+-}}{2 \sqrt{2}}\right)\right) \delta \sigma^{\dagger}-\frac{1}{2} \sigma \partial_{1} \delta \sigma^{\dagger}+\text { h.c.. }
$$

We note here that the vanishing of this term is consistent with the A-type boundary condition

$$
\sigma-e^{i \gamma} \sigma^{\dagger} \mid \partial \Sigma=0
$$

with Neumann conditions on the orthogonal combination. The angle $\gamma$ that this line in the complex $\sigma$ plane makes with the real axis is unfixed.

Finally, the equations of motion together with the supersymmetry variations of $\sigma$ require that $\lambda$ satisfy the boundary condition

$$
e^{i \gamma} \lambda_{+}+\lambda_{-} \mid \partial \Sigma=0
$$




\subsection{Bulk multiplets on the boundary}

In order to couple bulk and boundary multiplets supersymmetrically, we can decompose the boundary values of bulk multiplets into boundary multiplets.

The bulk vector multiplet restricts to a boundary vector multiplet and a real multiplet. The combination

$$
\nabla_{0}=\frac{i}{2}\left\{Q, Q^{\dagger}\right\}
$$

is a singlet under the B-type $\mathcal{N}=2$ supersymmetry. It will couple minimally to charged boundary matter fields according to their gauge charge under the bulk $U(1)$ symmetry.

The story for bulk chiral multiplets depends on the boundary conditions. If $\phi$ does not appear in a boundary superpotential, then:

$$
\begin{aligned}
\nabla_{1} \phi \mid \partial \Sigma & =0 \\
\eta \mid \partial \Sigma & =0 \\
\nabla_{1} \Theta \mid \partial \Sigma & =0 .
\end{aligned}
$$

The supersymmetry variations in Appendix A imply that $\phi$ and $\Theta$ form a boundary chiral multiplet, and $\eta$ and $F$ form a (trivial) boundary fermi multiplet. RG flow may induce a magnetic field coupling for the charged bulk fields at the boundary. This would make them analogous to the $\wp$ multiplet described above. Our considerations will be insensitive to this issue.

If $\phi$ is fixed by a boundary superpotential,

$$
\begin{aligned}
\nabla_{0} \phi \mid \partial \Sigma & =0 \\
\left.\Theta\right|_{\partial \Sigma} & =0 .
\end{aligned}
$$

In this case, the supersymmetry transformations imply that $\eta, F$ form a Fermi multiplet with a deformed chiral constraint $Q^{\dagger} \eta \sim \nabla_{1} \phi$.

\section{A boundary Lagrangian}

Next we write down the complete boundary interactions in an example. Take a Fermi multiplet $\beta$ such that $\bar{D} \beta_{a}=\sqrt{2} \wp E_{a}(\phi)$, with superpotential coupling

$$
\int_{\partial \Sigma} d x^{0} d \theta \beta_{a} \wp F^{a}(\phi)
$$


where $\wp$ is a boundary chiral multiplet such that $\bar{D}_{\wp}=0$. Then the kinetic and superpotential terms are:

$$
\begin{aligned}
S_{B}= & \sum_{a} \int d x^{0}\left[i\left(\beta_{a}^{\dagger} \nabla_{0} \beta_{a}-\nabla_{0} \beta_{a}^{\dagger} \beta_{a}\right)+\left|b_{a}\right|^{2}-\left|\wp E_{a}\right|^{2}\right. \\
& +\left(-i \beta_{a}^{\dagger} E_{a} \xi-i \beta_{a}^{\dagger} \wp \partial_{i} E_{a} \Theta^{i}+\text { h.c. }\right) \\
& \left.+\left(i \beta_{a} F^{a} \xi+i \beta_{a} \wp \sum_{i} \partial_{i} F_{a} \Theta^{i}+b_{a} \wp F^{a}+\text { h.c. }\right)\right]
\end{aligned}
$$

If we integrate out the boundary auxiliary fields, the boundary Lagrangian becomes:

$$
\begin{aligned}
\mathcal{L}_{\partial \Sigma} & =-\sum_{a}\left(2|\wp|^{2}\left|F^{a}\right|^{2}+|\wp|^{2}\left|E_{a}\right|^{2}\right) \\
& +i\left(\beta_{a}^{\dagger} \nabla_{0} \beta_{a}-\nabla_{0} \beta_{a}^{\dagger} \beta_{a}\right)-i\left(\nabla_{0} \wp^{\dagger}\right) \wp+i \wp^{\dagger} \nabla_{0} \wp \\
& +\left(-i \sum_{a} \beta_{a}^{\dagger}\left|E_{a}\right|^{2} \beta_{a}-i \beta_{a}^{\dagger} \wp \partial_{i} E_{a} \Theta^{i}+\text { h.c. }\right)+\left(-\sum_{a} \beta_{a}^{\dagger}\left|F^{a}\right|^{2} \beta_{a}+i \beta_{a} \wp \sum_{i} \partial_{i} F_{a} \Theta^{i}+\text { h.c. }\right) \\
& +\left(i \nabla_{1} p\right)^{\dagger} p+\eta_{p}^{\dagger} \Theta_{p}+\text { h.c. } \\
& +a_{0}\left(j_{s}-1\right) \\
& +\sigma\left(-\partial_{+} \sigma^{\dagger}-\frac{1}{\sqrt{2}}\left(i D-\frac{v_{+-}}{\sqrt{2}}\right)\right)+\sigma^{\dagger}\left(\partial_{-} \sigma+\frac{1}{\sqrt{2}}\left(i D+\frac{v_{+-}}{\sqrt{2}}\right)\right) \\
& +i\left(\lambda_{+}^{\dagger} \lambda_{+}-\lambda_{-}^{\dagger} \lambda_{-}\right)+\frac{1}{\sqrt{2}}\left(\sigma^{\dagger} t^{\dagger}-\sigma t\right)-\frac{i}{4}\left(\sigma-\sigma^{\dagger}\right)\left(\sum_{i} q_{i}\left|\phi_{i}\right|^{2}\right)
\end{aligned}
$$

The boundary term involving the bulk $p$ field will be explained in $§ 3.7$.

\subsection{Gauging the special symmetry}

As mentioned in $\S 2$, because the massless boundary fermions live in a vector bundle $V$, the Hilbert space on the boundary will transform in a sum of representations of the structure group of $V$. The $\beta$ s create $2^{r}$ states. To find the correct rank- $r$ Hilbert space at one end of the string, we make a projection.

To motivate this, consider $r$ coincident branes in flat space. We can model this by introducing $r$ free boundary fermi multiplets $\beta_{a}$. These fermions acting on the vacuum give $2^{r}=1+r+\left(\begin{array}{c}r \\ 2\end{array}\right)+\cdots$ states. To obtain an $r$-dimensional space of Chan-Paton factors, we project onto states with boundary fermion number one. 
Since our boundary fermions interact, boundary fermion number is no longer conserved. However, the boundary fermion number symmetry is replaced by the global boundary symmetry (2.5) mentioned above. We will project onto states which have charge 1 under this symmetry. This is accomplished by including a boundary vector, $a_{0}$, to act as a Lagrange multiplier

$$
\mathcal{L}_{\text {bdy }} a_{0}\left(j_{s}-1\right),
$$

where

$$
j_{s}=: \beta^{\dagger} \beta:-: \wp^{\dagger} \wp:
$$

is the boundary symmetry current.

\subsection{The Warner problem}

In the presence of a B-type boundary, the bulk worldsheet superpotential is no longer supersymmetric. Its $Q^{\dagger}$ variation is:

$$
\begin{aligned}
Q^{\dagger} \int_{\Sigma} \int d^{2} \theta W & =\int d x^{0} \Theta^{\bar{j} \dagger} \frac{i}{\sqrt{2}}\left(\eta_{i \bar{\jmath}} F^{i}-2 \bar{\partial}_{\bar{\jmath}} W^{\dagger}\right) \\
& \sim \int_{\partial \Sigma} Q W .
\end{aligned}
$$

This does not vanish even if we impose the equations of motion for $F$.

There are many options for dealing with this term. There is a family of boundary terms whose $Q^{\dagger}$ variation cancels the Warner term when the auxiliary fields in the bulk chiral multiplets satisfy their equations of motion. For example for the quintic with

$$
W=p G(\phi)
$$

one can add

$$
\Delta \mathcal{L}_{\mathrm{bdy}}=\int d \theta p \eta^{p \dagger}+\text { h.c. }
$$

which has

$$
Q^{\dagger} \int d \theta p \eta^{p^{\dagger}}=\int d \theta p F^{p^{\dagger}} .
$$

When the auxiliary field $F^{p}$ is evaluated on-shell, this gives

$$
Q^{\dagger} \Delta \mathcal{L}_{\text {bdy }} \sim \int d \theta p G(\phi)+\text { h.c. }
$$


which cancels the Warner variation. This solution works for any quasihomogeneous bulk superpotential.

Another approach is to set $W=0$ as one of the boundary conditions for the bulk chiral multiplets. Then the natural fermionic partner of this condition is precisely $\Theta^{i} \partial_{i} W=0$, and the boundary term (3.41) vanishes. This is natural in that it simply forces the boundaries of the worldsheet to lie in the CY hypersurface. This is equivalent to adding a neutral fermi multiplet $M=\mu+\theta m+\cdots$ such that

$$
\bar{D} M=-\frac{1}{\sqrt{2}}
$$

and writing a term of the form (3.19) with $S=W$. The supersymmetry variation of this action will cancel (3.41). This is the off-shell version of the fix used in [212]. The constraint (3.45) is the origin of the inhomogenous terms in the supersymmetry transformation in [212].

We believe that both these solutions will lead to sensible conformal theories.

\subsection{Symmetries of bulk and boundary fields}

\section{Bulk fields}

We will first review the global symmetries of the bulk fields [186]. There are leftand right-moving R-symmetries acting on the bulk chiral fields. Under the rightmoving R-symmetry the supersymmetry current $Q^{-}$has charge $1^{40}$; the Grassman variable $\theta^{+}$has charge 1 ; and the fields $\left(\psi_{+}, F, \sigma, \lambda_{-}\right)$have charges $(-1,-1,1,1)$. Under the left-moving R-symmetry, the supersymmetry current $Q^{+}$has charge 1 ; the Grassman variable $\theta^{-}$has charge 1 ; and the fields $\left(\psi_{-}, F, \sigma, \lambda_{+}\right)$have charges $(-1,-1,-1,1)$. These symmetries are non-anomalous so long as the sum of charges of the chiral multiplets vanishes.

The bulk superpotential generically breaks the R-symmetry. However, in absence of the superpotential there was an additional global symmetry under which each superfield $\Phi^{i}$ could be assigned arbitrary charge $k_{i}$. By adding this symmetry to the R-charge, we find new left and right moving $R$ symmetries under which $W$

40 The relation between the chiral bulk supercharges $Q^{ \pm}$and the B-type supercharges $Q, Q^{\dagger}$ is described in the Appendices. 
has left- and right- moving R-charge 1. This symmetry is diagonal; it must be added to both the left- and right-moving R-charges.

For B-type boundary SCFTs, only the sum of the R-charges is preserved:

$$
R_{t o t, i}=R_{+}+R_{-}+2 k_{i}
$$

\section{Boundary fields}

Since bulk and boundary fields are coupled, both will be charged under the conserved R-symmetry. The R-charges of the boundary fields can be deduced from the charges of bulk fields which restrict to the boundary. One will again have to compose symmetries of the boundary theory with the naive R-symmetry to make a symmetry which is respected by the interactions. There is no anomaly in $(0+1)$ dimensions so this symmetry will survive quantization. We will assume that this $U(1)_{R}$ flows to the expected R-symmetry of the infrared $\mathcal{N}=2$ superconformal algebra.

\section{Sheafy variables}

Given a description of a Chan-Paton bundle as the cohomology of an arbitrary sequence of sums of line bundles, a prescription was given in [178] for making a linear sigma model of the type detailed above. However, [178] focused on bundles which have constant fiber dimension over the entire Calabi-Yau manifold.

One linear model for branes of finite codimension, i.e. sheaves with nonconstant fiber dimension over the CY, was described in $§ 2.3$. Simply add a boundary fermi multiplet $\gamma$ of charge $-d \equiv-\operatorname{degree}(S(\phi))$ and add a boundary superpotential

$$
\int d \theta \gamma S(\phi)
$$

This produces a boundary energy $\sim|S(\phi)|^{2}$ which confines the string boundary to the hypersurface. This boundary theory would describe a D-brane localized at $S(\phi)=0$. In this section we present another formulation of this model, which is useful because it makes e.g. the possibility of transitions between Higgs and Coulomb branches in the D-brane moduli space more transparent. 
Introduce a boundary fermi multiplet $\beta$ of bulk gauge charge $-d$, and a gauge neutral boundary chiral multiplet $\wp$, and project as usual onto the charge one sector of the boundary symmetry (2.5). Instead of (4.1), add

$$
\int d \theta \wp \beta S(\phi) .
$$

The resulting vacuum equations set $\wp S(\phi)=0$. Away from the locus $\mathcal{S} \equiv\{S=$ $0\}$ this is accomplished by setting $\wp=0 ; \beta$ is massed up by $\xi$. Away from the hypersurface, therefore, there is no way to satisfy the boundary charge projection on the vacuum manifold. When $S=0$, on the other hand, there is no constraint on $\beta$ or $\wp$, but the charge projection picks out a single Chan-Paton state which we will describe in detail below. The resulting D-brane is the sheaf which is the cohomology of the sequence

$$
0 \rightarrow \mathcal{O}(-d) \stackrel{S(\phi)}{\longrightarrow} \mathcal{O} \rightarrow 0
$$

Away from $\mathcal{S}$ there is no cohomology; over the hypersurface, the map degenerates and a single cohomology element appears at adjacent nodes.

This method of building a brane on a subcycle has also been described from the spacetime point of view, e.g. in $[215,216,217]$. At large volume, adjacent vector bundles in a sequence have opposite-signed D6-brane charge. The map between them represents the open string tachyon. The sequence above describes a D6-brane with D4-brane charge, and an anti-D6 brane; they annihilate to leave a D4-brane behind.

To translate between the two descriptions of hypersurfaces we have given, one can think of $\gamma$ as a boundary-gauge-symmetry-invariant composite of $\wp$ and $\beta$. We refer to the description using $\wp$ and $\beta$ as "sheafy variables" and employ it in the next section when we discuss transitions in open-string moduli space.

The reader may be confused by the fact that in the sheafy description there seem to be two Chan-Paton states localized at the zero set $\mathcal{S}$, one created by $\beta$ and one created by $\wp^{\dagger}$. That this is not the case can be seen by calculating the cohomology of $Q^{\dagger}$ acting on boundary states. As explained in more detail in $\S 6$, this yields the number of massless open string states in the Ramond sector.

Consider an example where there is just a single bulk chiral field $z$ parametrizing a copy of $\mathbb{C}$. Take $\mathcal{S} \equiv\{S(z)=z=0\}$, i.e. a D0-brane at the origin, as 
described by the sequence (4.3). Finally, introduce an additional space-filling brane with a single trivial Chan-Paton state, i.e. a D2-brane. We will find the massless strings stretching between our brane made by the sequence (4.3) and the D2-brane. Let us start with a vacuum of the interesting end of the string which satisfies

$$
0=\Theta|0\rangle=\beta^{\dagger}|0\rangle=\wp|0\rangle
$$

Imposing the boundary charge projection, an arbitrary state in the Hilbert space is

$$
|\psi\rangle=f_{1}\left(z, z^{\dagger}\right) \beta|0\rangle+f_{2}\left(z, z^{\dagger}\right) \beta \Theta^{\dagger}|0\rangle+f_{3}\left(z, z^{\dagger}\right) \wp^{\dagger}|0\rangle+f_{4}\left(z, z^{\dagger}\right) \wp^{\dagger} \Theta^{\dagger}|0\rangle .
$$

Now we want to solve for $Q^{\dagger}|\psi\rangle=0$ modulo $Q^{\dagger}$-exact states. Reducing to zeromodes, the relevant terms in $Q^{\dagger}$ are

$$
Q^{\dagger}=\Theta^{\dagger} \bar{\partial}+\beta \wp z
$$

Acting on the first term on the RHS of (4.5), we find:

$$
Q^{\dagger} f_{1}(z, \bar{z}) \beta|0\rangle=i \bar{\partial} f_{1} \Theta^{\dagger} \beta|0\rangle
$$

If $f_{1}(z)$ is holomorphic, this state is in the kernel of $Q^{\dagger}$. Furthermore, $Q^{\dagger}$ acts on the third term on the RHS (4.5) to give:

$$
Q^{\dagger} f_{3}(z) \wp^{\dagger}|0\rangle=i z f_{3}(z) \beta|0\rangle
$$

Finally, the second and fourth term on the RHS of (4.5) are Q-exact.

Thus, within cohomology,

$$
f_{1}(z) \simeq f_{1}(z)+z f_{3}(z)
$$

The cohomology representatives live in the $\operatorname{ring} \mathbb{C}[z] /\langle z\rangle$, the local ring at $z=0$ (c.f. [218]). There is a single cohomology generator localized at $z=0$. This is our state, up to $Q^{\dagger}$ descendants.

We can see even more explicitly that the string endpoint is stuck at the origin and has a single Chan-Paton factor by solving for the ground state wavefunction of the worldsheet zero modes, which is killed by both $Q^{\dagger}$ and $Q$. To do this exactly we need the IR effective action for the zero modes. In this example the bulk theory is a free scalar field theory and the relevant perturbations lie entirely on the boundary. 
If we assume that the RG flow does not add anything to the bulk kinetic terms (as appears to be the case in related work [202]), and if the boundary superpotential satisfies a nonrenormalization theorem similar to that for the bulk superpotential, then the only effect of the RG flow in the presence of the relevant boundary perturbation (4.2) will be to change the anomalous dimension of the superpotential. As the term (4.2) is relevant along the RG flow, it will be dressed with a factor $M^{\alpha}$, where $M \rightarrow \infty$ in the IR and $\alpha$ is positive.

Altering the supercharges accordingly, the ground state should satisfy:

$$
\begin{aligned}
& 0=Q^{\dagger}\left|\psi_{0}\right\rangle=\left(-i \bar{\partial} f_{1}-i M^{\alpha} z f_{4}\right) \Theta^{\dagger} \beta|0\rangle+\left(-i \bar{\partial} f_{3}\right) \Theta^{\dagger} \wp^{\dagger}|0\rangle+M^{\alpha}\left(i z f_{3}\right) \beta|0\rangle \\
& 0=Q\left|\psi_{0}\right\rangle=\left(-i f_{1} \bar{z}-i M^{\alpha} \partial f_{4}\right) \wp^{\dagger}|0\rangle+M^{\alpha}\left(i \partial f_{2}\right) \beta|0\rangle+\left(-i \bar{z} f_{2}\right) \wp^{\dagger} \Theta^{\dagger}|0\rangle .
\end{aligned}
$$

where we have ignored time derivatives since $Q^{\dagger}=Q=0$ implies $H=0$. The first equation in (4.10) requires that $f_{3}$ is holomorphic, with support only at $z=0$; thus $f_{3}=0$. Similarly, the second equation leads to $f_{2}=0$. Rotational invariance implies that the lowest-energy state $\left|\psi_{0}\right\rangle$ depends only on $(z \bar{z})$. Therefore,

$$
\left|\psi_{0}\right\rangle \sim A(M) e^{-M^{\alpha}|z|^{2}}\left(\beta|0\rangle+\wp^{\dagger} \Theta^{\dagger}|0\rangle\right) .
$$

In the infrared, as $M \rightarrow \infty$, the ground state will become a delta function at $\mathcal{S}=\{z=0\}$. This has also been observed in [219].

\subsection{Unions and intersections}

In this section, we explain a number of ways to combine two boundary linear sigma models to make another. For argument, we discuss two transverse hypersurfaces of a CY defined by $S_{1}(\phi)=0$ and $S_{2}(\phi)=0$ with degrees $d_{1}$ and $d_{2}$.

\section{Intersections}

Here are three distinct ways to make the strings end on the intersection $\left\{S_{1}=S_{2}=0\right\}:$

1. As described in $\S 2$, add a boundary superpotential:

$$
W=\gamma_{1} S_{1}+\gamma_{2} S_{2}
$$

The vacuum energy $\left|S_{1}\right|^{2}+\left|S_{2}\right|^{2}$ only vanishes on the intersection. 
2. The tensor product of two sheaves has support on the intersection of the support of the two sheaves. Given the resolution of two sheaves by a sequence of line bundles, there is a formula for such a resolution of the tensor product whose derivation from the LSM is described in Appendix C. In this example, it gives:

$$
\begin{gathered}
0 \rightarrow \mathcal{O} \stackrel{S_{1}}{\longrightarrow} \mathcal{O}\left(d_{1}\right) \rightarrow 0 \\
0 \rightarrow \mathcal{O} \stackrel{S_{2}}{\longrightarrow} \mathcal{O}\left(d_{2}\right) \rightarrow 0 \\
= \\
0 \rightarrow \mathcal{O} \stackrel{\left[\begin{array}{l}
S_{1} \\
S_{2}
\end{array}\right]}{\longrightarrow} \mathcal{O}\left(d_{1}\right) \oplus \mathcal{O}\left(d_{2}\right) \stackrel{\left[-S_{2}, S_{1}\right]}{\longrightarrow} \mathcal{O}\left(d_{1}+d_{2}\right) \rightarrow 0
\end{gathered}
$$

The field content is a neutral $\wp$, two $\beta$ 's of charges $d_{1,2}$, and a $\tilde{\wp}$ of charge $-\left(d_{1}+d_{2}\right)$ with the constraints on the $Q$ 's and $Q^{\dagger}$ 's determined by the sequence (c.f. [178]).

3. There is another sheafy description of this intersection. Take as boundary fields $\beta_{1,2}$ and $\wp_{1,2}$, coupled via the boundary superpotential:

$$
W=\wp_{1} \beta_{1} S_{1}+\wp_{2} \beta_{2} S_{2}
$$

and project on separate boundary symmetries for each of $\beta_{1}, \wp_{1}$ and $\beta_{2}, \wp_{2}$. Away from the intersection the boundary system cannot satisfy one or the other charge projection while staying on the vacuum manifold.

\section{Unions}

Three analogous models for the union $\left\{S_{1}=0\right\} \cup\left\{S_{2}=0\right\}$ are:

1. The scheme variables union is simply to use a single boundary fermion of charge $-d_{1}-d_{2}$ and add

$$
W=\gamma S_{1}(\phi) S_{2}(\phi)
$$

The boundary energy is then $\left|S_{1} S_{2}\right|^{2}$ and it vanishes if one lies on either hypersurface. This gives a scheme-theoretic description in the following sense. Notice that if $S_{1}$ and $S_{2}$ are the same, one gets multiple Chan-Paton sectors. In this sense, this boundary linear model keeps track of the fact that a pair of 
coincident branes is a non-reduced scheme. Related observations were made in $[220]$.

2. As with a single hypersurface, we can describe this in "sheafy" variables. By this we mean replace $\gamma$ by a fermion $\beta$ and a boson $\wp$, gauge the new boundary symmetry, and add

$$
W=\wp \beta S_{1}(\phi) S_{2}(\phi)
$$

3. While tensoring sheaves intersects their supports, adding them gives a sheaf whose support is the union. Simply adding together the sequences we find:

$$
\begin{gathered}
0 \rightarrow \mathcal{O} \stackrel{S_{1}}{\longrightarrow} \mathcal{O}\left(d_{1}\right) \rightarrow 0 \\
\bigoplus \\
0 \rightarrow \mathcal{O} \stackrel{S_{2}}{\longrightarrow} \mathcal{O}\left(d_{2}\right) \rightarrow 0 \\
= \\
0 \rightarrow \mathcal{O} \oplus \mathcal{O}^{\left[S_{1}, S_{2}\right]} \mathcal{O}\left(d_{1}\right) \oplus \mathcal{O}\left(d_{2}\right) \rightarrow 0
\end{gathered}
$$

The superpotential is the same as (4.14). But if there is just a single charge projection, we obtain the union of the two branes because the string ends on one brane or the other. So this is really the analog of the third way of intersecting - we just do one charge projection instead of two. This is also consistent with the fact that if only one of the boundary symmetries is gauged, then there is one left over which acts as the second $U(1)$ spacetime gauge symmetry - the branes can move independently.

\section{Applications and consequences}

\subsection{Monodromy in closed-string moduli space}

The open string linear sigma model is an ideal tool for thinking about transport of branes in closed string moduli space. Choose some boundary field content to make a particular D-brane in the IR. If we move along a closed path in Kähler moduli space, we come back to the same bulk LSM, but with possibly different boundary data. This determines an action of the monodromy group on the branes themselves, and not just on their charges. Such a refinement of the action of the monodromy 
group on branes has also been observed in the approach of $[215,221,216]$ where branes are considered as objects in the derived category.

In the case of the quintic, there are three monodromy generators [222]. At large volume, the imaginary part of the complexified Kähler form - represented in the linear model by the worldsheet theta angle - has periodicity $2 \pi$. A shift by $2 \pi$ at large $r$ is a noncontractible loop and should generate the "large-volume monodromy." A loop about $r=0$ should generate a monodromy which corresponds to the monodromy about the conifold point in the mirror CY. Finally, at large negative $r$ a shift of $\theta$ by $2 \pi$ is also a noncontractible loop in the closed string GLSM. Since $r \rightarrow-\infty$ describes the Gepner point which is a $\mathbb{Z}_{5}$ orbifold point in the moduli space, this shift of $\theta$ should generate the $\mathbb{Z}_{5}$ monodromy action about the Gepner point.

\section{Large radius}

Consider a single D6-brane on the quintic, modeled by a single neutral boundary fermion which carries charge 1 under the gauged boundary symmetry. The pertinent terms in the boundary action are

$$
\mathcal{L}_{b d y}\left(\frac{\theta}{2 \pi} v_{0}+j v_{0}+\left(j_{s}-1\right) a_{0}\right)
$$

where $v_{0}$ is the bulk worldsheet gauge field, $j$ is the bulk gauge current, $j_{s}$ is the boundary symmetry current, and $a_{0}$ is the boundary vector field in (3.39).

Now, shifting $\theta \rightarrow \theta+2 \pi \alpha$ adds to this

$$
\delta \mathcal{L}=\alpha v_{0},
$$

(this is only gauge invariant if $\alpha$ is an integer multiple of $2 \pi$ ). This is equivalent to shifting the bulk gauge charges of all fields by their boundary charge:

$$
j \rightarrow j+\alpha j_{s} .
$$

In our example, this means that when $\alpha=1$, we can remove the term (5.2) by giving the boundary fermion bulk gauge charge 1 . But this shifts the chern classes of the bundle; in particular, it adds one unit of four-brane charge. This is the expected large-radius monodromy. 
This is the right answer for any boundary field content. Adding the boundary symmetry generator to the gauge charge has the effect of tensoring the bundle with the line bundle $\mathcal{O}(1)$ over the projective space. This is precisely the effect of moving the NS B-field through one period [222,182].

\section{Other monodromy generators}

An understanding of the other independent monodromy generator requires an analysis of the effective theory at the conifold singularity. Work is in progress in this direction. Here we restrict ourselves to a few suggestive observations.

Firstly, even at large negative $r, \theta \rightarrow \theta+2 \pi$ acts by adding the boundary current to the gauge current. So it seems that the idempotence of the LG monodromy action is related to the $\mathbb{Z}_{5}$-valuedness of the worldsheet gauge charge.

Secondly, it generates the expected monodromy on the branes following the discussion in [195]. A "fractional brane" in their model corresponds to a brane whose Chan-Paton factors have $\mathbb{Z}_{5}$ charge under the orbifold group, corresponding to the different irreducible representations of $\mathbb{Z}_{5}$. The quantum symmetry at the orbifold point rotates these irreps and so shifts all of the charges by 1 mod 5 .

Finally, the trivial representation is believed to correspond to the D6-brane at the Gepner point [195]. This is the same description as at large radius. The fact that the D6-brane has no monodromy about $r=0$ suggests that we have the right description at the Gepner point.

\subsection{Degenerations and Singularities}

Singularities in the CFT moduli space are especially important. They provide a window into nonperturbative physics, and can give rise to the singularities of the spacetime superpotential which are expected in $\mathcal{N}=1$ supergravity [223,190]. In the closed string GLSM, singularities of the CFT appear at points in the GLSM moduli space where the vacuum manifold becomes noncompact [186]. This should also hold true in the open string case. Furthermore, apparent singularities in the open string moduli space, such as "small instanton" singularites, signal enhanced gauge symmetries or the existence of a branch structure in the moduli space.

\section{Singularities in the closed string moduli space}

For closed strings, singularities in the complex structure moduli space occur when $G$ is not transverse; then, as in [186], there is a branch where $p$ and some 
of the $\phi$ s diverge with zero energy cost. If there are boundary terms of the form (3.19), where $S$ depends only on the bulk fields, $\phi^{i}$ will not always be able to diverge consistent with $S^{A}(\phi)=0$, so the $D$-term keeps $p$ from diverging. The boundary CFT will not be singular (i.e. the D-brane physics will be smooth) at many points where the closed string CFT would be singular.

If $S=0$ is consistent with the nonzero $\phi^{i}$ necessary for the existence of the non-compact $p$ branch, then the full boundary CFT will be singular. In other words, there can be singularities in the relevant boundary CFT as well as the closed string CFT when the D-brane intersects the closed string singularity.

Singularities in the Kähler moduli space occur when $\sigma$ has a noncompact branch. For the closed GLSM on the quintic, this occurs at $r=\theta=0$. It is an interesting question whether D-brane physics is singular at this point - see for example [181]. If the Chan-Paton factors for a given state have nontrivial gauge charge then the answer is uncertain. When $\sigma$ is large all of the charged fields have masses of order $|\sigma|^{2}$. The charged boundary fermions create a constant electric field in the bulk. Competing effects exist: on the one hand one expects screening of the bulk electric field via Schwinger pair production, on the other hand the mass of the bulk charged fields (which must be pair-produced to provide the screening) grows quickly down the $\sigma$ branch. It would be interesting to disentangle the physics of the potential singularity by performing a delicate analysis of the IR limit (as was done for closed strings in [190]).

\section{Singularities from boundary fields?}

If the boundary field $\wp$ has no potential, one might worry that a noncompact branch develops and the CFT is singular. For example, in the Calabi-Yau phase, $\wp$ is usually frozen to zero by the term $\sum_{a}|\wp|^{2}\left|F^{a}\right|^{2}$, which gives $\wp$ a large mass since the $F^{a}$ are generically nonvanishing at $S=0$ in the Calabi-Yau. However, one could choose a bundle with singular points, where the $F^{a}$ all vanish on $S=0$. Even more uncomfortably, in the Landau-Ginzburg phase $\phi=F=0$. It would be bizarre if the CFT was singular in an open set in the moduli space.

However a $\wp$ branch does not exist. The boundary charge projection gives a finite number of states in the Hilbert space of boundary fields. The $\wp$ field is a 
"quantum dimension" in the target space, and cannot cause divergences in the path integral. The integral over $a_{0}$ projects onto:

$$
j_{s}=\sum_{a}: \beta_{a}^{\dagger} \beta_{a}:-: \wp^{\dagger} \wp:=1
$$

Since the $\beta$ s are fermionic, the positive contribution in (5.4) is bounded; therefore (when (5.4) can be satisfied), $\wp$ contributes a finite volume factor to the path integral and does not give any new branches to the path integral.

\section{Enhanced gauge symmetries}

Despite the absence of a $\wp$ branch, there is significant physics when a bundle degenerates. If a locus $\mathcal{D}$ exists such that for $\phi_{\star}^{i} \in \mathcal{D}, F^{a}\left(\phi_{\star}\right)=G\left(\phi_{\star}\right)=0$, then in addition to the bundle $V$, we get a sheafy variables description of a lowerdimensional brane - namely a sheaf with support over $\mathcal{D}$. The small instanton limit [224] of the D0-D4 system is the classic example of such a degeneration.

In addition, we can sometimes tune parameters such that additional global symmetries arise, in addition to (2.5) which rotates all $\beta$ 's and $\wp$ 's oppositely. Then additional spacetime gauge symmetries should appear.

\section{Small Instanton "Singularities"}

The "sheafy variables" construction of D-branes allows us to easily study transitions between branches of D-brane moduli spaces. First we will study two D2-branes filling orthogonal complex lines in a flat $\mathbb{C}^{3}$. The "Higgs branch" can occur when they lie in a common $\mathbb{C}^{2}$ and can be deformed into a single D2-brane; the "Coulomb branch" occurs when they are separated along the complex line orthogonal to both of them. If we replaced $\mathbb{C}^{3}$ by $T^{6}$ this would be the $\mathrm{T}$-dual of the small instanton singularity. We will then proceed to a direct study of small instantons in the D0-D4 system.

Consider the linear model which flows to the Coulomb branch. We take as

boundary fields $\beta, \tilde{\beta}, \wp_{1,2}, \beta^{\prime}, \tilde{\beta}^{\prime}$. We wish to describe a background with one twobrane located at $z_{1}=0, z_{3}=0$, and the other at $z_{2}=0, z_{3}=a$. 
Using the "sheafy variables" description, the complex defining this configuration is

$$
\begin{aligned}
& \left(\begin{array}{c}
0 \rightarrow \mathcal{O} \stackrel{z_{1}}{\rightarrow} \mathcal{O} \rightarrow 0 \\
\otimes \\
z_{3} \rightarrow \mathcal{O} \rightarrow \mathcal{O} \rightarrow 0
\end{array}\right) \bigoplus\left(\begin{array}{c}
0 \rightarrow \mathcal{O} \stackrel{z_{2}}{\rightarrow} \mathcal{O} \rightarrow 0 \\
\otimes \\
z_{3}-a \\
0 \rightarrow \mathcal{O} \rightarrow \mathcal{O} \rightarrow 0
\end{array}\right) \\
& =
\end{aligned}
$$

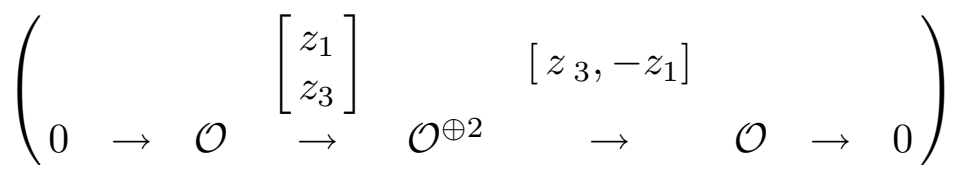

$$
\begin{aligned}
& \bigoplus
\end{aligned}
$$

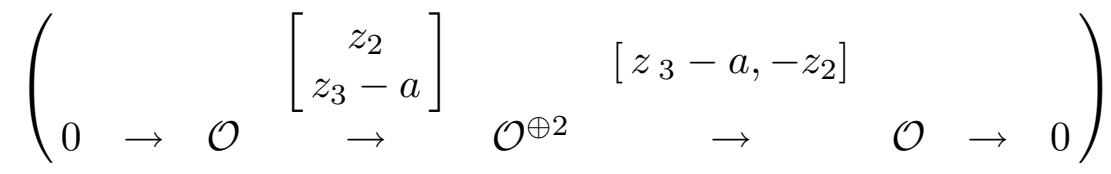

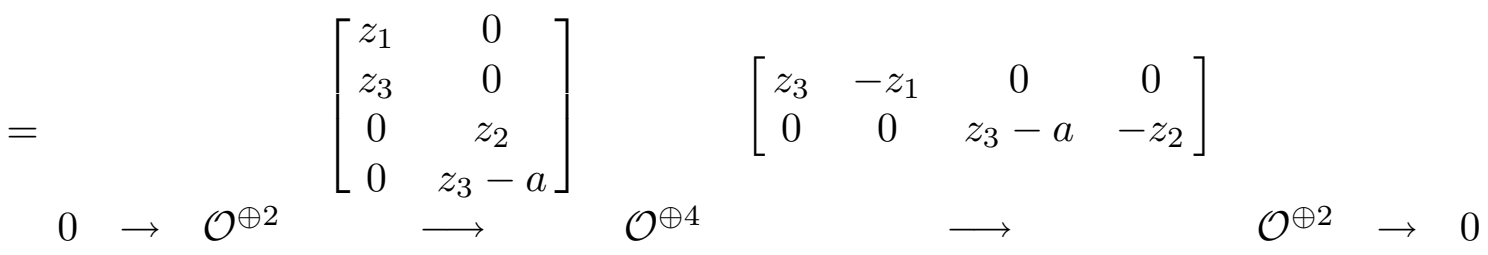

In other words, the deformed chiral constraints are

$$
\begin{array}{lccc}
Q^{\dagger} \beta=0 & Q^{\dagger} \wp_{1}^{\dagger}=z_{1} \beta & Q^{\dagger} \wp_{2}^{\dagger}=z_{3} \beta & Q^{\dagger} \beta^{\prime}=z_{3} \wp_{1}^{\dagger}-z_{1} \wp_{2}^{\dagger} \\
Q^{\dagger} \tilde{\beta}=0 & Q^{\dagger} \tilde{\wp}_{1}^{\dagger}=z_{2} \tilde{\beta} & Q^{\dagger} \tilde{\wp}_{2}^{\dagger}=\left(z_{3}-a\right) \tilde{\beta} & Q^{\dagger} \tilde{\beta}^{\prime}=\left(z_{3}-a\right) \tilde{\wp}_{1}^{\dagger}-z_{2} \tilde{\wp}_{2}^{\dagger}
\end{array}
$$

and the on-shell supersymmetry transformations are

$$
\begin{array}{cccc}
Q^{\dagger} \beta^{\dagger}=-z_{1} \wp_{1}-z_{3} \wp_{2} & Q^{\dagger} \wp_{1}=-z_{3}{\beta^{\prime}}^{\dagger} & Q^{\dagger} \wp_{2}=z_{1}{\beta^{\prime}}^{\dagger} & Q^{\dagger}{\beta^{\prime}}^{\dagger}=0 \\
Q^{\dagger} \tilde{\beta}^{\dagger}=-z_{2} \tilde{\wp}_{1}-\left(z_{3}-a\right) \tilde{\wp}_{2} & Q^{\dagger} \tilde{\wp}_{1}=-\left(z_{3}-a\right) \tilde{\beta}^{\prime \dagger} & Q^{\dagger} \tilde{\wp}_{2}=z_{2} \tilde{\beta}^{\prime \dagger} & Q^{\dagger} \tilde{\beta}^{\prime \dagger}=0
\end{array}
$$

One can search for marginal deformations of the system in one of two ways. Either one can look for candidate superpotential terms annihilated by $Q^{\dagger}$ (using the unperturbed EOM and values for the auxiliary fields); or one can look for consistent perturbations of the off-shell deformed chiral constraints and add no explicit superpotential term. The two approaches yield equivalent results; here we will take the latter.

We are interested in deformations which preserve the orientation of the branes and more generally the structure of the configuration at infinity, so we only allow deformations of the maps of the complex which are constant independent of 
z. Deformations linear in $z$ would generally alter the orientation of the branes, and higher-order polynomials would cause even more drastic changes in the brane geometry.

For $a \neq 0$ there are no constant deformations of the system other than the obvious ones corresponding to moving the twobrane moduli around on the Coulomb branch.

When $a$ vanishes, however, one can find other consistent perturbations of the deformed chiral constraints, or equivalently deformations of the complex preserving nilpotence of the differential $Q^{\dagger}$.

Specifically, for vanishing $a$ the most general set of constants one can add to the maps of the complex is:

$$
\begin{aligned}
& {\left[\begin{array}{cc}
z_{1} & \epsilon \\
z_{3} & 0 \\
\epsilon^{\prime} & z_{2} \\
0 & z_{3}
\end{array}\right] \quad\left[\begin{array}{cccc}
z_{3} & -z_{1} & 0 & -\epsilon \\
0 & -\epsilon^{\prime} & z_{3} & -z_{2}
\end{array}\right]} \\
& 0 \rightarrow \mathcal{O}^{\oplus 2} \quad \longrightarrow \quad \mathcal{O}^{\oplus 4} \quad \longrightarrow \quad \mathcal{O}^{\oplus 2} \quad \rightarrow \quad 0
\end{aligned}
$$

We will now show that for $a=0$ the effect of this perturbation will be to merge the two branes into a single brane covering the locus $z_{3}=0, z_{1} z_{2}=\epsilon \epsilon^{\prime}$. After adding this deformation of the complex, the deformed chiral constraints become

$$
\begin{array}{llll}
Q^{\dagger} \beta=0 & Q^{\dagger} \wp_{1}^{\dagger}=z_{1} \beta+\epsilon_{1} \tilde{\beta} & Q^{\dagger} \wp_{2}^{\dagger}=z_{3} \beta & Q^{\dagger} \beta^{\prime}=z_{3} \wp_{1}^{\dagger}-z_{1} \wp_{2}^{\dagger}-\epsilon \tilde{\wp}_{2}^{\dagger} \\
Q^{\dagger} \tilde{\beta}=0 & Q^{\dagger} \tilde{\wp}_{1}^{\dagger}=z_{2} \tilde{\beta}+\epsilon^{\prime} \beta & Q^{\dagger} \tilde{\wp}_{2}^{\dagger}=z_{3} \tilde{\beta} & Q^{\dagger} \tilde{\beta}^{\prime}=z_{3} \tilde{\wp}_{1}^{\dagger}-z_{2} \tilde{\wp}_{2}^{\dagger}-\epsilon^{\prime} \wp_{2}^{\dagger}
\end{array}
$$

and the on-shell supersymmetry transformations are

$$
\begin{array}{llll}
Q^{\dagger} \beta^{\dagger}=-z_{1} \wp_{1}-z_{3} \wp_{2}-\epsilon^{\prime} \tilde{\wp}_{1} & Q^{\dagger} \wp_{1}=-z_{3} \beta^{\prime \dagger} & Q^{\dagger} \wp_{2}=z_{1} \beta^{\prime \dagger}+\epsilon^{\prime} \tilde{\beta}^{\prime \dagger} & Q^{\dagger} \beta^{\prime \dagger}=0 \\
Q^{\dagger} \tilde{\beta}^{\dagger}=-z_{2} \tilde{\wp}_{1}-z_{3} \tilde{\wp}_{2}-\epsilon \wp_{1} & Q^{\dagger} \tilde{\wp}_{1}=-z_{3} \tilde{\beta}^{\prime \dagger} & Q^{\dagger} \tilde{\wp}_{2}=z_{2} \tilde{\beta}^{\prime \dagger}+\epsilon \beta^{\prime \dagger} & Q^{\dagger} \tilde{\beta}^{\prime \dagger}=0
\end{array}
$$

The condition for the $Q$ and $Q^{\dagger}$ variations of all $\wp$ fields to vanish is

$$
\begin{array}{r}
z_{3}\left(\beta, \tilde{\beta}, \beta^{\prime \dagger}, \tilde{\beta}^{\prime \dagger}\right)=0 \\
\left(z_{1} z_{2}-\epsilon \epsilon^{\prime}\right)\left(\beta, \tilde{\beta}, \beta^{\prime \dagger}, \tilde{\beta}^{\prime \dagger}\right)=0 .
\end{array}
$$

So we see that this deformation moves the support of the brane to the irreducible variety defined by

$$
z_{3}=0, \quad z_{1} z_{2}=\epsilon \epsilon^{\prime}
$$

which represents the Higgs branch of this D2-D2 system. 
There is a shortcut to finding the locus of the twobrane. If one takes a generic point in the base space, the matrices defining the complex are all of full rank. Their rank is reduced, leading to nonzero cohomology, exactly when all two by two subdeterminants vanish. This occurs at the locus $z_{3}=0, z_{1} z_{2}=\epsilon \epsilon^{\prime}$.

\section{Higgs-Coulomb transition in the D0-D4 system}

The merging of two $D 2$-branes is $T$-dual to the Coulomb-Higgs transition in the 0-4 system. We can also describe this transition directly.

A linear sigma model for a zerobrane separated from a pair of fourbranes is

$$
0 \rightarrow \mathcal{O} \stackrel{d_{1}}{\rightarrow} \mathcal{O}^{\oplus 5} \stackrel{d_{2}}{\rightarrow} \mathcal{O}^{\oplus 5} \stackrel{d_{3}}{\rightarrow} \mathcal{O} \rightarrow 0
$$

with

$$
\begin{gathered}
d_{1} \equiv\left[\begin{array}{c}
z_{1} \\
z_{2} \\
z_{3}-L \\
0 \\
0
\end{array}\right] \\
d_{2} \equiv\left[\begin{array}{ccccc}
0 & z_{3}-L & -z_{2} & 0 & 0 \\
L-z_{3} & 0 & z_{1} & 0 & 0 \\
z_{2} & -z_{1} & 0 & 0 & 0 \\
0 & 0 & 0 & z_{3} & 0 \\
0 & 0 & 0 & 0 & z_{3}
\end{array}\right] \\
d_{3} \equiv\left[\begin{array}{lllll}
z_{1}, & z_{2}, & z_{3}-L & 0, & 0
\end{array}\right] .
\end{gathered}
$$

For generic $L$ the only marginal operators one can add simply correspond to shifts in the positions of the branes. However for $L=0$ one can deform the complex to:

$$
\begin{aligned}
d_{1} \equiv\left[\begin{array}{c}
z_{1} \\
z_{2} \\
z_{3} \\
-B_{1} \\
-B_{2}
\end{array}\right] \\
d_{2} \equiv\left[\begin{array}{ccccc}
0 & z_{3} & -z_{2} & 0 & 0 \\
-z_{3} & 0 & z_{1} & 0 & 0 \\
z_{2} & -z_{1} & 0 & C_{1} & C_{2} \\
0 & 0 & B_{1} & z_{3}+E_{11} & E_{12} \\
0 & 0 & B_{2} & E_{21} & z_{3}+E_{22}
\end{array}\right] \\
d_{3} \equiv\left[\begin{array}{lllll}
z_{1}, & z_{2}, & z_{3}, & -C_{1}, & -C_{2}
\end{array}\right]
\end{aligned}
$$


for $B, C, E$ which satisfy

$$
\left[\begin{array}{ll}
C_{1}, & C_{2}
\end{array}\right]\left[\begin{array}{l}
B_{1} \\
B_{2}
\end{array}\right]=\left[\begin{array}{ll}
E_{11} & E_{12} \\
E_{21} & E_{22}
\end{array}\right]\left[\begin{array}{l}
B_{1} \\
B_{2}
\end{array}\right]=\left[\begin{array}{ll}
C_{1}, & C_{2}
\end{array}\right]\left[\begin{array}{ll}
E_{11} & E_{12} \\
E_{21} & E_{22}
\end{array}\right]=0
$$

The general solution to these constraints consists of a unit doublet $\left(u_{1}, u_{2}\right)$ and three complex numbers $b, c, e$ :

$$
\left[\begin{array}{l}
B_{1} \\
B_{2}
\end{array}\right]=b\left[\begin{array}{l}
u_{1} \\
u_{2}
\end{array}\right] ; \quad\left[\begin{array}{ll}
C_{1}, & C_{2}
\end{array}\right]=c\left[\begin{array}{ll}
u_{2}, & -u_{1}
\end{array}\right] ; \quad\left[\begin{array}{ll}
E_{11} & E_{12} \\
E_{21} & E_{22}
\end{array}\right]=e\left[\begin{array}{cc}
u_{1} u_{2} & -u_{1}^{2} \\
u_{2}^{2} & -u_{1} u_{2}
\end{array}\right]
$$

We will now assume generic values of $b, c, e, u$. There is never cohomology at the first or last node; neither $d_{1}$ nor $d_{3}$ ever has a kernel. $d_{2}$ always has determinant zero, which is to be expected since $d_{1}$ has one-dimensional image, so $d_{2}$ must have at least one-dimensional kernel.

There will be cohomology at the second and third nodes only when the dimension of the kernel of $d_{2}$ is two or higher, the criterion for which is the vanishing of all twenty-five 4 by 4 subdeterminants of $d_{2}$. We find that the matrix of subdeterminants is

$$
z_{3}^{2} \cdot\left[\begin{array}{ccccc}
-b c u_{1} u_{2}, & b c u_{1}^{2}, & c u_{1} z_{3}, & -c u_{1} z_{2}, & c u_{1} z_{1} \\
-b c u_{2}^{2}, & b c u_{1} u_{2}, & c u_{2} z_{3}, & -c u_{2} z_{2} & c u_{2} z_{1} \\
-b u_{2} z_{3} & b u_{1} z_{3} & z_{3}^{2} & -z_{2} z_{3} & z_{1} z_{3} \\
b u_{2} z_{2} & -b u_{1} z_{2} & -z_{2} z_{3} & z_{2}^{2} & -z_{1} z_{2} \\
-b u_{2} z_{1} & b u_{1} z_{1} & z_{1} z_{3} & -z_{1} z_{2} & z_{1}^{2}
\end{array}\right]
$$

Specifically, it factorizes as $z_{3}^{2}$ times a matrix which never vanishes. (The entry in the upper left hand corner, for instance, is constant and nonzero). This means that there are two fibers along the locus $z_{3}=0$ (rather than a single fiber, since the zero is doubled), and no extra fibers at special subloci of $z_{3}=0$. The interpretation is that we have dissolved a zerobrane into a smooth instanton field in the pair of fourbranes. (Note that the value of $e$ drops out of the subdeterminants and we can set it to zero in what follows.)

We can see this even more directly by examining the cohomology restricted to $z_{3}=0$. Setting $e=0$ and computing the kernel of $d_{2}$ we have

$$
d_{2} \equiv\left[\begin{array}{ccccc}
0 & 0 & -z_{2} & 0 & 0 \\
0 & 0 & z_{1} & 0 & 0 \\
z_{2} & -z_{1} & 0 & C_{1} & C_{2} \\
0 & 0 & B_{1} & 0 & 0 \\
0 & 0 & B_{2} & 0 & 0
\end{array}\right]\left[\begin{array}{c}
\beta_{1} \\
\beta_{2} \\
\beta_{3} \\
\beta_{4} \\
\beta_{5}
\end{array}\right]=0
$$




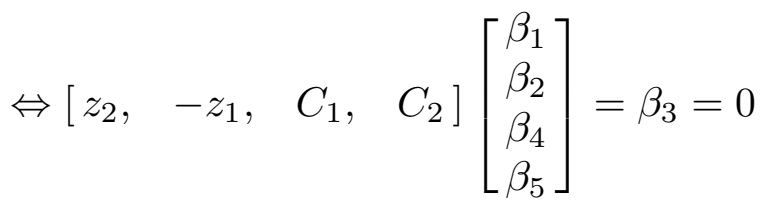

Furthermore, the massless $\beta$ 's are in the kernel of $d_{1}^{\dagger}$, restricted to $z_{3}=0$. That is,

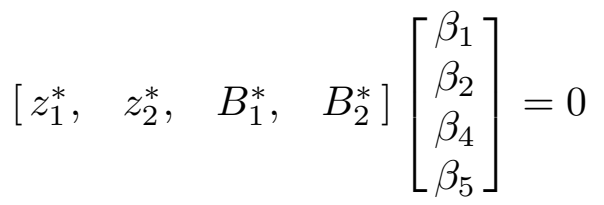

In other words, the vector $\left[\beta_{1}, \beta_{2}, \beta_{4}, \beta_{5}\right]$ is a harmonic representative of the cohomology of the complex

$$
\begin{aligned}
& {\left[\begin{array}{l}
z_{1} \\
z_{2} \\
B_{1} \\
B_{2}
\end{array}\right] \quad\left[\begin{array}{llll}
z_{2}, & -z_{1}, & C_{1}, & C_{2}
\end{array}\right]} \\
& 0 \rightarrow \mathcal{O} \rightarrow \mathcal{O}^{\oplus 4} \quad \rightarrow \quad \rightarrow \quad \text { O } \rightarrow 0
\end{aligned}
$$

with

$$
\left[\begin{array}{cc}
C_{1}, & C_{2}
\end{array}\right]\left[\begin{array}{l}
B_{1} \\
B_{2}
\end{array}\right]=0
$$

But the ADHM construction of a single $U(2)$ instanton in $\mathbb{R}^{4}$ works precisely by defining a holomorphic bundle as the cohomology of this same complex. So we have directly demonstrated the ability of a D0 brane to dissolve into D4 branes by open string worldsheet arguments, without any reference to the brane worldvolume gauge theory.

This whole computation should generalize without undue complication to the process of dissolving $k$ instantons in $N$ fourbranes to make a smooth $k$-instanton field in a $U(N)$ gauge theory on $\mathbb{R}^{4}$.

\subsection{Marginal stability transitions: A local worldsheet model}

While many of the quantities which are reliably computed in the open string LSM are independent of the worldsheet FI parameter, it is clear that important aspects of the physics of B-type D-branes do depend on the Kähler moduli. The most striking example is marginal stability transitions, which occur at special loci in the Kähler moduli space. Determining when a transition occurs for a given set of 
brane charges is a delicate problem which has come under intense recent investigation (see e.g. $[182,183,184,215]$ ). Since the topological B-model is insensitive to the Kähler parameters, the occurence of such transitions is not transparent in the linear model. However, we can understand the local physics of these transitions in the linear model in a simple way. The local model we present below is a good candidate to describe the generic worldsheet behavior in the vicinity of such a transition.

A local model for the spacetime physics of a D-brane undergoing a marginal stability transition is the Fayet model [225]. This is easily seen in the A-model $[1,226]$, where the physics can be reduced to a problem involving intersecting branes. ${ }^{41}$ The Fayet model is a four-dimensional $\mathcal{N}=1$ field theory with a $U(1)$ gauge symmetry and a single charged field. (In the brane system, there is also a center of mass vector field gauging a $U(1)$ under which all fields are neutral.) The D-term takes the form

$$
D=|\phi|^{2}-\xi
$$

When the FI parameter $\xi$ is positive, there is a supersymmetric vacuum at nonzero $\phi$; the gauge symmetry is broken by this vev and there is a mass gap. For $\xi<0$, there is no way to make $D$ vanish; supersymmetry is broken, and the gauge symmetry is preserved. Classically, the gauge multiplet $\left(A_{\mu}, \lambda_{\alpha}\right)$, and the fermion partner $\psi_{\alpha}$ of $\phi$ are all massless, while $\phi$ is massive. In realizations of this transition in B-type brane systems, $\xi$ is to be identified with a nontrivial function of the Kähler parameter $t=\frac{\theta}{2 \pi}+i r$. There is a supersymmetric brane for $\xi>0$ and the brane decays on the locus $\xi\left(t, t^{\dagger}\right)=0$ in Kähler moduli space.

This simplest model of the spacetime physics is reproduced by the simplest possibility in the LSM. Add a brane-antibrane pair to the linear model - i.e. add a boundary chiral multiplet $\wp$ and a boundary fermi multiplet $\beta$.

To model the behavior when $\xi$ is small and positive, add the operator

$$
f(\xi) \int d \theta \wp \beta
$$

to the boundary action ( $f$ is some nontrivial function of $\xi$, which vanishes when $\xi=0)$. The $Q^{\dagger}$ complex then takes the form

$$
0 \rightarrow\left\{\beta^{\dagger} \wp^{\dagger}\right\} \rightarrow\left\{\begin{array}{c}
\wp^{\dagger} \wp \\
\beta^{\dagger} \beta
\end{array}\right\} \rightarrow\{\beta \wp\} \rightarrow 0
$$

41 In the B-model, these transitions are related to a chamber structure in the moduli space of stable bundles [227]. 
At generic points when $\xi>0, f$ is non-vanishing. The last map from R-charge 0 to R-charge 1 is then onto, and there is no cohomology beyond the center of mass vector at $\mathrm{R}$-charge 0 . We have a supersymmetric vacuum with the mass gap (for modes other than the decoupled $U(1))$ that we expect. The R-symmetry of the CFT to which we flow must preserve (5.27), so the operator $\beta \wp$ has unit R-charge.

Now consider the behavior at $\xi=0$. Since $f(\xi)=0$ at this point, the maps in (5.28) degenerate and we get a cohomology generator at R-charge zero (in addition to the center-of-mass gaugino $\mathbf{1}=\beta^{\dagger} \beta-\wp^{\dagger} \wp$ ) which we identify as a new gaugino. Its image under spectral flow is a new massless vector. There is also new cohomology at R-charge 1 of the form $\beta \wp$ which we identify with the vertex operator for $\psi_{\alpha}$, the fermi component of the charged chiral multiplet. The R-charge of this operator is still unity, and so spectral flow generates from it the vertex operator for massless scalar $\phi$. One can show, using the equation

$$
\left[\beta^{\dagger} \beta, \wp \beta\right]=-\wp \beta
$$

that the string created by this vertex operator indeed carries unit charge under the new vector field, as expected.

Now, what happens as one moves past the spacetime transition point, to the $\xi<0$ region of parameter space of the Fayet model? At $\xi=0$, the model enjoys an extra unbroken $U(1)$ global symmetry - one can rotate $\beta$ and $\wp$ independently. There is no longer a unique candidate for the $U(1)_{R}$ symmetry which appears in the IR $\mathcal{N}=2$ superconformal algebra. We can hypothesize that when $\xi$ is made slightly negative, the R-charge is a linear combination of the two $U(1)$ s under which $\beta \wp$ has charge $>1$. We then find that the scalar $\phi$ obtains a tree-level mass since the conformal weight of the image of $V_{\psi}$ under spectral flow will be different from 1. The operator $\beta \wp$ is still in the $Q^{\dagger}$ cohomology, so $\psi_{\alpha}$ remains massless.

This scenario will be realized in the following situation. When $\xi>0$, the $\phi$ field has two gauge-inequivalent vacua (the true vacuum, and the tachyonic vacuum at $\langle\phi\rangle=0)$. Associated with these two vacua are two different boundary CFTs. In the CFT of the supersymmetric vacuum, where the tachyonic perturbation, $\int d \theta \beta \wp$, has been turned on, the conserved R-charge must be such that $\beta \wp$ has unit R-charge. 


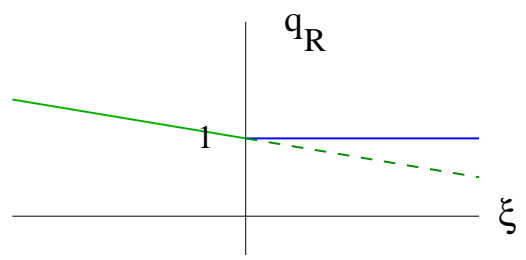

Fig. 17: The R-charge, $q_{R}$, of the operator $\wp \beta$ varies with the spacetime FI coefficient $\xi$. The dashed line indicates the conserved R-charge in the CFT of the tachyonic vacuum at $\langle\phi\rangle=0$.

In the unperturbed CFT of the tachyonic $\langle\phi\rangle=0$ vacuum, there is an extra global boundary $U(1)$ symmetry under which (if necessary by adding to it a multiple of the gauged boundary current) only $\beta$ is charged. Therefore the conserved Rcharge in the IR theory can be some linear combination of the bulk R-current and the $\beta$ number current with $r$-dependent (and hence $\xi$-dependent) coefficients determined by the boundary RG flow. It is this gauge-symmetric vacuum (and hence this CFT) which describes the brane at $\xi<0$, and so the R-charge of $\wp \beta$ can vary with $\xi$ when $\xi$ becomes negative.

It would be very interesting to understand in detail, from a microscopic point of view, the appearance of this local model in various D-brane decay processes. It is quite plausible that not only generic marginal stability transitions, but also generic variations of the worldvolume spectrum on a given D-brane, can be accomplished through the judicious addition of brane/anti-brane pairs in this manner.

\section{Massless worldvolume fields}

\subsection{Generalities}

In this section we compute the massless worldvolume spectrum in a particular example. Our linear model flows to the worldsheet-supersymmetric Ramond sector of strings ending on these branes, and it is the states in this sector which are annihilated by $L_{0}$ that we determine. As in $[215,216,217]$ it should be understood that we are looking at branes in the topological B-model, and the spectrum of strings we are computing corresponds to the massless open (fermionic) strings stretching between such branes. The relation of these "topological" branes to physical branes is not always straightforward: the supersymmetric branes in the topological model satisfy the physical F-flatness conditions but not necessarily the physical D-flatness conditions. The question of which topological branes satisfy the latter condition is 
equivalent to understanding marginal stability, and loci of marginal stability are not manifest in the linear model (and indeed still need to be determined on a more or less case by case basis). In some special cases, e.g. half-supersymmetric D-branes on $K 3$, the enhanced supersymmetry makes it easier to infer properties of the physical branes from the topological model, so after some generalities we will specialize to an example involving branes on $K 3$.

To find the massless open string states, we can study the supersymmetric ground states in the Ramond sector. In this sector, the two unbroken supercharges $Q$ and $Q^{\dagger}$ anti-commute to the Ramond-sector Hamiltonian:

$$
\left\{Q, Q^{\dagger}\right\}=2 L_{0}
$$

By (6.1) and standard results in Hodge theory, we can find the supersymmetric ground states by computing the $Q^{\dagger}$ cohomology. Since the FI terms are $Q$ exact, we can ignore their effects in this computation.

We will find it convenient to compute the cohomology of $Q^{\dagger}$ acting on operators.

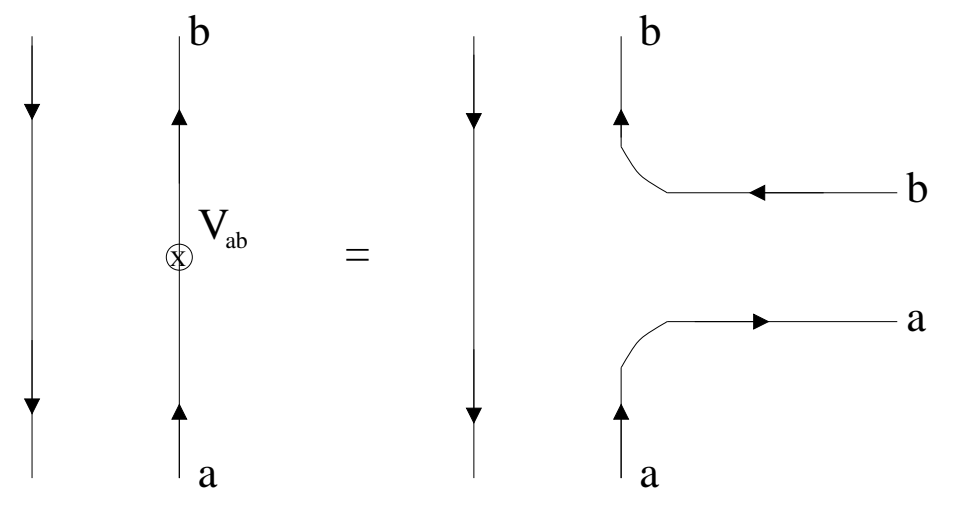

Fig. 18: In the conformal limit, emission of a string stretched between branes is the same as insertion of a boundary-condition-changing vertex operator, $V_{a b} \sim \beta_{b}^{\dagger} \beta_{a}$.

Since, as illustrated in fig. 18, there is a 1-1 correspondence between the open string states and associated boundary condition changing operators, this does not constitute a loss of generality. 
Our example consists of three D4-

\begin{tabular}{|c|c|c|c|}
\multicolumn{1}{c}{ Field } & \multicolumn{1}{c}{$q_{G}$} & \multicolumn{1}{c}{$q_{R}$} & \multicolumn{1}{c}{$q_{S}$} \\
\hline$\phi^{i}$ & 1 & $\frac{1}{2}$ & 0 \\
\hline$\Theta^{i}, \eta^{i}$ & 1 & $-\frac{1}{2}$ & 0 \\
\hline$p$ & -4 & 0 & 0 \\
\hline$\Theta_{p}, \eta_{p}$ & -4 & -1 & 0 \\
\hline$\beta_{a}$ & 1 & $-\frac{1}{2}$ & 1 \\
\hline$\wp$ & -4 & 0 & -1 \\
\hline
\end{tabular}

Table 1: The charges of the relebranes on the quartic K3, with the bundle $V$ defined by the sequence (2.10) with $m=4$ and $\left\{n_{a}\right\}=\{1,1,1,1\}$. There is a boundary superpotential coupling $\int d \theta \beta_{a} F^{a}(\phi) \wp$. The table at left displays the gauge charges, R-charge, and boundary global charges of all of the relevant fields. The bulk vector is neutral under all of these transformations. vant fields.

In the GLSM for this simple monad on K3, our supercharge takes the form

$$
\begin{aligned}
& Q^{\dagger}=Q_{\mathrm{bulk}}^{\dagger}+Q_{\partial}^{\dagger} \\
& =\int d x\left\{\nabla_{0} \phi_{i} \Theta^{i \dagger}+\nabla_{1} \phi_{i} \eta^{i \dagger}+2 p \partial_{i} G \eta^{i}+\nabla_{0} p \Theta_{p}^{\dagger}+\nabla_{1} p \eta_{p}^{\dagger}+2 G \eta_{p}\right. \\
& +\lambda_{+}\left(-\partial_{+} \sigma+\frac{1}{\sqrt{2}}\left(i D+\frac{v_{+-}}{\sqrt{2}}\right)\right)+\lambda_{-}\left(\partial_{-} \sigma^{\dagger}-\frac{1}{\sqrt{2}}\left(i D-\frac{v_{+-}}{\sqrt{2}}\right)\right) \\
& \left.\quad+\delta(\partial \Sigma)\left(\wp \beta_{a} F^{a}(\phi)+\eta_{p}^{\dagger} p\right)\right\} .
\end{aligned}
$$

- We will only be interested in operators which are invariant under the gauged special boundary symmetry (so that they map states in the charge-one sector back into the same sector). Furthermore, if we are not going to restrict ourselves to a phase of the theory where the bulk $U(1)$ is higgsed and integrate out the bulk gauge field, we must throw away operators which are not $U(1)$ invariant. We want to find the spectrum of massless spacetime fields. The fermionic parts of such multiplets arise from Ramond vertex operators whose internal parts have R-charge 0 or 1 . The former map via spectral flow to NS-sector operators of conformal weight 0 and hence lead to spacetime vectors. Ramond states of unit R-charge flow spectrally to NS operators of unit dimension which give the scalar components of spacetime chiral multiplets. 
- The equation of motion of the boundary vector field $a_{0}$ is imposed as an operator equation of motion. So we impose the boundary Gauss' Law,

$$
: \beta_{a}^{\dagger} \beta_{a}:-: \wp^{\dagger} \wp:= \pm 1
$$

where the \pm depends on the orientation of the boundary component, on our operators.

- In order to be boundary-symmetry-invariant, an operator has to contain an even number of boundary fields. Operators without any boundary fields are just bulk operators restricted to the boundary and we ignore them. Included among these is the identity operator, which creates the center-of-mass vector. We claim that operators containing four or more boundary fields do not create any states beyond those made by operators bilinear in the fiber fields. We can see this by making the state-operator correspondence more explicit as follows. The only subtlety involved is the vacuum degeneracy (present even in the NS sector) arising from the boundary fermion and boson zero modes. To resolve this, we choose a reference state,

$$
|0\rangle_{+} \otimes|0\rangle_{-} .
$$

Here $|0\rangle_{ \pm}$are vacua of the two ends of the string satisfying

$$
0=\beta_{a}|0\rangle_{-}=\wp^{\dagger}|0\rangle_{-}=\beta_{a}^{\dagger}|0\rangle_{+}=\wp|0\rangle_{+} .
$$

So we have arbitarily picked out a state which is not in the boundary Hilbert space of the ends of the string because it does not satisfy the boundary charge projection. The state corresponding to an operator is obtained by acting with that operator on this vacuum and projecting onto the subspace satisfying the charge projection. For example, an operator of the form $\beta_{a}^{\dagger} \beta_{b}$ makes the state with the - end of the string in sector $a$, and the + end of the string in sector $b$. Acting on this state, the only independent boundary operators invariant under the boundary symmetry are of the form

$$
\wp^{\dagger} \beta^{\dagger}, \wp^{\dagger} \wp, \beta^{\dagger} \beta, \wp \beta .
$$

Operators which are least quartic in the fiber fields are made by acting with one of these operators again. Using the charge projection (6.3) this will always give zero or a state created by a quadratic operator. 
- It is convenient to divide up $Q^{\dagger}$ into

$$
Q^{\dagger}=Q_{0}^{\dagger}+Q_{1}^{\dagger}
$$

where $Q_{0}^{\dagger}$ includes only the parts depending on derivatives of bulk fields. By a zig-zag argument of the type appearing in [206] we can compute the cohomology of $Q^{\dagger}$ by computing the cohomology of $Q_{1}^{\dagger}$ in the cohomology of $Q_{0}^{\dagger}$. This tells us that we can leave out any non-holomorphic dependence on $\phi$ and we can leave out $\Theta$ 's and their daggers because any operator with $\Theta$ - or $\phi^{\dagger}$-dependence is a $Q^{\dagger}$ descendant.

- We discard the vector multiplet from our cohomology calculation. It is massive at large $|r|$, where its effect is to impose Gauss' law and its supersymmetric completions. Combined with the fact that the result of this calculation is independent of worldsheet FI terms (which are $Q^{\dagger}$ descendants), this means that we may safely neglect it. A more rigorous justification for this awaits future work.

- Operators containing the bulk field $p$, but not containing its partner $\eta_{p}^{\dagger}$ are in the image of the supercharge because of the term

$$
\delta(\partial \Sigma) \eta_{p}^{\dagger} p
$$

added to the supercharge to solve the Warner problem.

Schematically, the structure of the action of $Q^{\dagger}$ on the pertinent operators is:

$$
0 \rightarrow\left\{R_{(l)}^{a}(\phi) \beta_{a}^{\dagger} \wp^{\dagger}\right\} \rightarrow\left\{\begin{array}{c}
S_{(l+3)}(\phi) \wp^{\dagger} \wp \\
S_{(l+3)}^{a b}(\phi) \beta_{a}^{\dagger} \beta_{b}
\end{array}\right\} \rightarrow\left\{T_{(l+6)}^{a}(\phi) \beta_{a \wp}\right\} \rightarrow 0,
$$

where the notation means that $R_{(l)}^{a}(\phi)$ is a homogeneous polynomial in $\phi$ of degree $l$ (and likewise for $S \mathrm{~s}$ and $T \mathrm{~s}$ ).

In order that these operators be gauge invariant we need $l=-3$. This means that the first node is trivial, and at the second node the polynomials are just constants. At the third node, the polynomials are cubic and these operators are exactly of the form of marginal deformations of the superpotential. Accordingly, they have R-charge 1 . The image of $Q^{\dagger}$ from the R-charge zero operators at the previous node consists of operators of the form

$$
R_{a b} F^{a}(\phi) \beta_{b} \wp,
$$


where $R_{a b}$ is a constant and $F^{a}$ is the section of $\bigoplus \mathcal{O}\left(n_{a}\right)$ appearing in the boundary superpotential. This tells us that in $Q^{\dagger}$ cohomology,

$$
T_{(3)}^{a}(\phi) \simeq T_{(3)}^{a}+R_{a b} f^{b}(\phi) .
$$

Since there are twenty independent degree three monomials in four variables, we find $4(20-4)=64$ elements of cohomology at R-charge 1 from this part of the $Q^{\dagger}$ complex.

At R-charge 0 , the only operator in $\operatorname{ker}\left(\mathrm{Q}^{\dagger}\right)$ is

$$
: \beta_{a}^{\dagger} \beta_{a}:-: \wp^{\dagger} \wp:=j_{s}
$$

which by the boundary Gauss' law (6.3) is the identity operator which creates (the fermion partner of) the center-of-mass vector field.

We note here that the brane worldvolume Higgs mechanism has a very natural implementation in this complex. Cohomology can appear at adjacent nodes which have R-charge 0 and 1 respectively, resulting in a new vector field and a new charged multiplet descending to zero mass.

Note that this framework could as easily have been applied directly to the large-radius phase of the theory. In that case, we would have simply set $p=0$ to its vacuum value and ignored its massive fluctuations. One finds the same operators representing the cohomology, and the calculation essentially reduces to the classical mathematics of deformation theory. It would be interesting to perform an analogous calculation directly in the Landau-Ginzburg effective field theory, after integrating out the $p$ field and gauge multiplet.

\section{Consequences of the index theorem}

For the Chern-classes of the $S U(3)$ bundle $V$ one finds $c_{1}(V)=0$ and $c_{2}(V)=$ 24. A theorem of Mukai [228] tells us that the dimension of the moduli space of such a bundle is related directly to the index of $Q^{\dagger}$, and in this example has (quaternionic) dimension 64. Therefore, one expects the brane spectrum to include 64 massless hypermultiplets, in agreement with our result.

The index of $Q^{\dagger}$ is not directly related to the dimension of the brane moduli space for branes on Calabi-Yau threefolds. In those examples, the index of $Q^{\dagger}$ is still invariant under smooth deformations of the closed string and open string moduli, but there are adjacent nodes in the complex representing scalar states which may pair up with each other while preserving the index of the $Q^{\dagger}$ complex. Note that the index of $Q^{\dagger}$ is invariant under addition of brane-antibrane pairs in the linear sigma model. 


\section{Future Directions}

Many interesting issues arise in the study of D-branes on Calabi-Yau spaces, and our formalism might be usefully extended to address a number of them. Here, we close by mentioning several subjects for future exploration:

- One expects that generic branes will cross lines of marginal stability in the $(r, \theta)$ plane. While a mechanism for implementing brane decays in the linear model was discussed in $§ 5.3$, it would be very interesting to derive the form of these loci of marginal stability directly in the linear model. This would presumably involve a direct calculation of the relevant spacetime central charge.

- The physics of D-branes at singular points in their moduli space (where even the boundary CFT becomes ill-defined) should be tractable in this approach. For closed strings, the new non-compact branches which arise in the linear model at singular points in moduli space were shown in e.g. [190] to allow one to reproduce detailed calculations about divergent terms in the spacetime effective action. A similar story may well arise here.

- A related question: At certain loci in moduli space, wrapped D-branes become massless spacetime states. This happens for instance at the mirror conifold point in the Kähler moduli space of the quintic, where the wrapped D6 brane becomes massless. What is the behavior of the worldvolume theory on the brane in such a limit? What happens as one makes extremal transitions to new branches of moduli space where such wrapped branes become fundamental string states?

- We have confined ourselves to discussing $B$-type branes in this chapter; but similar methods could work for $A$-type branes as well (for earlier work in this direction see $[191,192]$, for later work in this direction see [211]). As discussed in $[18,39,229]$ there are expected to be intricate, disc-instanton generated superpotentials for $A$ type branes. It would be interesting to formulate a linear sigma model description of such branes in which the instanton sum was computable. In a somewhat analogous problem with more supersymmetry, Morrison and Plesser did succeed in reproducing closed string instanton effects directly in the linear sigma model [210]. For recent work in this direction, see [230].

- Finally, any microscopically consistent model with wrapped, space-filling D-branes will have to include orientifolds (or anti-branes) as well to cancel RR tadpoles. It 
will be interesting to study new phenomena that arise in generalizing this kind of worldsheet description to models with orientifolds and/or antibranes.

\section{Acknowledgements}

We are grateful to Jacques Distler, Kentaro Hori, Greg Moore, Michael Peskin, Joe Polchinski, Eva Silverstein, Lenny Susskind and Nick Warner for helpful discussions. Some discussions of boundary conditions were originally developed in unpublished work by Thiagarajan Jayaraman, A.L., Hirosi Ooguri and Tapobrata Sarkar. They have since appeared (with the absent authors' blessings) in [192]. A.L. would like to thank the other authors and Suresh Govindarajan for collaboration and discussions. Parts of this work were undertaken while S.K. and A.L. enjoyed the hospitality of the Aspen Center for Physics and the Institute for Theoretical Physics at Santa Barbara. This work was supported in part by the DOE under contract DE-AC03-76SF00515. The work of S.K. is also supported by a David and Lucile Packard Foundation Fellowship for Science and Engineering, an Alfred P. Sloan Foundation Fellowship, and National Science Foundation grant PHY0097915. The work of J.M. was supported in part by the Department of Defense NDSEG Fellowship program.

\section{Appendix A. Transformation properties of bulk supermultiplets}

Throughout this chapter we use $Q=\frac{Q_{+}-Q_{-}}{\sqrt{2}}$ and its conjugate as the generators of the unbroken B-type supersymmetry. $S=\frac{Q_{+}+Q_{-}}{\sqrt{2}}$ and its conjugate generate the supersymmetry transformations broken by the boundary theory.

The $(2,2)$ algebra is

$$
\begin{gathered}
\left\{Q_{ \pm}, Q_{ \pm}\right\}=0 \quad\left\{Q_{ \pm}, Q_{\mp}\right\}=0 \\
\left\{Q_{ \pm}, Q_{ \pm}^{\dagger}\right\}=2 P_{ \pm} \\
\left\{Q_{+}, Q_{-}^{\dagger}\right\}=2 Z \quad\left\{Q_{-}, Q_{+}^{\dagger}\right\}=2 Z^{\dagger} \\
{\left[Q_{ \pm}, P_{\mp}\right]=-i \sqrt{2} q \lambda_{\mp}^{\dagger} \quad\left[Q_{ \pm}^{\dagger}, P_{\mp}\right]=-i \sqrt{2} q \lambda_{\mp}}
\end{gathered}
$$

where $Z$ acts by multiplication by $q \sigma^{\dagger}$ on a field of charge $q$ and $P_{ \pm}=-i D_{ \pm}$is the gauge covariant momentum (but does not contain $\sigma \mathrm{s}$ ). Note that $\left[\nabla_{ \pm}, Q\right]=$ $\left[\nabla_{ \pm}, Q^{\dagger}\right]=0$. 
In the following, our supercharges act by graded commutation. The $(2,2)$

supersymmetry transformations of the fields in a chiral multiplet, $\phi$, of $U(1)$ gauge

charge $a$ in Wess-Zumino gauge are

$$
\begin{array}{rrl}
Q \phi=-i \Theta & Q^{\dagger} \phi=0 & Q \phi^{\dagger}=0 \quad Q^{\dagger} \phi^{\dagger}=-i \Theta^{\dagger} \\
S \phi=-i \eta & S^{\dagger} \phi=0 & S \phi^{\dagger}=0 \quad S^{\dagger} \phi^{\dagger}=-i \eta^{\dagger} \\
Q \Theta=0 & Q^{\dagger} \Theta=2 \nabla_{0} \phi & Q \Theta^{\dagger}=2 \nabla_{0} \phi^{\dagger} \quad Q^{\dagger} \Theta^{\dagger}=0 \\
S \Theta=-F & S^{\dagger} \Theta=2 \nabla_{1} \phi & S \Theta^{\dagger}=2\left(\nabla_{1} \phi\right)^{\dagger} \quad S^{\dagger} \Theta^{\dagger}=-F^{\dagger} \\
Q \eta=F & Q^{\dagger} \eta=2 \tilde{\nabla}_{1} \phi & Q \eta^{\dagger}=2\left(\tilde{\nabla}_{1} \phi\right)^{\dagger} \quad Q^{\dagger} \eta^{\dagger}=F^{\dagger} \\
S \eta=0 & S^{\dagger} \eta=2 \tilde{\nabla}_{0} \phi & S \eta^{\dagger}=2 \tilde{\nabla}_{0} \phi^{\dagger} \quad S^{\dagger} \eta^{\dagger}=0 \\
Q F=0 & Q F^{\dagger}=2 i\left(\left(\tilde{\nabla}_{1} \Theta\right)^{\dagger}-\nabla_{0} \eta^{\dagger}\right)+2 q \phi^{\dagger}\left(\lambda_{+}+\lambda_{-}\right) \\
Q^{\dagger} F=2 i\left(\tilde{\nabla}_{1} \Theta-\nabla_{0} \eta\right)+2 q \phi\left(\lambda_{+}^{\dagger}+\lambda_{-}^{\dagger}\right) & Q^{\dagger} F^{\dagger}=0 \\
S F=0 & S F^{\dagger}=2 i\left(\tilde{\nabla}_{0} \Theta^{\dagger}-\left(\nabla_{1} \eta\right)^{\dagger}\right)+2 q \phi^{\dagger}\left(\lambda_{+}-\lambda_{-}\right) \\
S^{\dagger} F=2 i\left(\tilde{\nabla}_{0} \Theta-\nabla_{1} \eta\right)+2 q \phi\left(\lambda_{+}^{\dagger}-\lambda_{-}^{\dagger}\right) & S^{\dagger} \Theta^{\dagger}=-F^{\dagger}
\end{array}
$$


The vector multiplet fields transform as:

$$
\begin{aligned}
& Q \sigma=i \lambda_{+}^{\dagger} \quad Q^{\dagger} \sigma=-i \lambda_{-} \quad Q \sigma^{\dagger}=-i \lambda_{-}^{\dagger} \quad Q^{\dagger} \sigma^{\dagger}=i \lambda_{+} \\
& S \sigma=i \lambda_{+}^{\dagger} \quad S^{\dagger} \sigma=i \lambda_{-} \quad S \sigma^{\dagger}=i \lambda_{-}^{\dagger} \quad S^{\dagger} \sigma^{\dagger}=i \lambda_{+} \\
& Q \lambda_{+}=-\partial_{+} \sigma^{\dagger}-\frac{1}{\sqrt{2}}\left(i D-\frac{v_{+-}}{\sqrt{2}}\right) \quad Q^{\dagger} \lambda_{+}^{\dagger}=-\partial_{+} \sigma+\frac{1}{\sqrt{2}}\left(i D+\frac{v_{+-}}{\sqrt{2}}\right) \\
& Q^{\dagger} \lambda_{+}=0 \quad S^{\dagger} \lambda_{+}=0 \quad Q \lambda_{+}^{\dagger}=0 \quad S \lambda_{+}^{\dagger}=0 \\
& S \lambda_{+}=-\partial_{+} \sigma^{\dagger}+\frac{1}{\sqrt{2}}\left(i D-\frac{v_{+-}}{\sqrt{2}}\right) \quad S^{\dagger} \lambda_{+}^{\dagger}=-\partial_{+} \sigma-\frac{1}{\sqrt{2}}\left(i D+\frac{v_{+-}}{\sqrt{2}}\right) \\
& Q \lambda_{-}=\partial_{-} \sigma+\frac{1}{\sqrt{2}}\left(i D+\frac{v_{+-}}{\sqrt{2}}\right) \quad Q^{\dagger} \lambda_{-}^{\dagger}=\partial_{-} \sigma^{\dagger}-\frac{1}{\sqrt{2}}\left(i D-\frac{v_{+-}}{\sqrt{2}}\right) \\
& Q^{\dagger} \lambda_{-}=0 \quad S^{\dagger} \lambda_{-}=0 \quad Q \lambda_{-}^{\dagger}=0 \quad S \lambda_{-}^{\dagger}=0 \\
& S \lambda_{-}=-\partial_{-} \sigma+\frac{1}{\sqrt{2}}\left(i D+\frac{v_{+-}}{\sqrt{2}}\right) \quad S^{\dagger} \lambda_{-}^{\dagger}=-\partial_{-} \sigma^{\dagger}-\frac{1}{\sqrt{2}}\left(i D-\frac{v_{+-}}{\sqrt{2}}\right) \\
& Q v_{+-}=-i\left(\partial_{+} \lambda_{-}^{\dagger}+\partial_{-} \lambda_{+}^{\dagger}\right) \quad Q^{\dagger} v_{+-}=-i\left(\partial_{+} \lambda_{-}+\partial_{-} \lambda_{+}\right) \\
& S v_{+-}=-i\left(\partial_{+} \lambda_{-}^{\dagger}-\partial_{-} \lambda_{+}^{\dagger}\right) \quad S^{\dagger} v_{+-}=-i\left(\partial_{+} \lambda_{-}-\partial_{-} \lambda_{+}\right) \\
& Q i \sqrt{2} D=i\left(\partial_{+} \lambda_{-}^{\dagger}-\partial_{-} \lambda_{+}^{\dagger}\right) \quad Q^{\dagger} i \sqrt{2} D=-i\left(\partial_{+} \lambda_{-}-\partial_{-} \lambda_{+}\right) \\
& S i \sqrt{2} D=i\left(\partial_{+} \lambda_{-}^{\dagger}+\partial_{-} \lambda_{+}^{\dagger}\right) \quad S^{\dagger} i \sqrt{2} D=-i\left(-\partial_{+} \lambda_{-}+\partial_{-} \lambda_{+}\right)
\end{aligned}
$$

\section{Appendix B. Boundary superspace}

We can exponentiate the supersymmetry transformations to define a superspace formalism. We use the same bulk superspace coordinates as in [186]. In this language the action of supersymmetry on superfields is generated by:

$$
\begin{aligned}
\delta \Phi & =\left(\epsilon^{\alpha} Q_{\alpha}-\bar{\epsilon}^{\alpha} Q_{\alpha}^{\dagger}\right) \Phi \\
& =\left(-\epsilon_{-} Q_{+}+\epsilon_{+} Q_{-}+\bar{\epsilon}_{-} Q_{+}^{\dagger}-\bar{\epsilon}_{+} Q_{-}^{\dagger}\right) \Phi
\end{aligned}
$$

where $\epsilon^{\alpha}$ is the Grassman parameter; the spinor indices are raised and lowered by 
the antisymmetric tensor $\epsilon_{\alpha \beta}$ as in [186]. Recall that

$$
\begin{aligned}
& Q_{+}=\frac{\partial}{\partial \theta^{+}}+i \bar{\theta}^{+} \partial_{+} \\
& Q_{-}=\frac{\partial}{\partial \theta^{-}}+i \bar{\theta}^{-} \partial_{-} \\
& Q_{+}^{\dagger}=-\frac{\partial}{\partial \bar{\theta}^{+}}-i \theta^{+} \partial_{+} \\
& Q_{-}^{\dagger}=-\frac{\partial}{\partial \bar{\theta}^{-}}-i \theta^{-} \partial_{-}
\end{aligned}
$$

where

$$
\partial_{ \pm}=\partial_{0} \pm \partial_{1}
$$

We can similarly define superspace derivatives:

$$
\begin{aligned}
& D_{ \pm}=\frac{\partial}{\partial \theta^{ \pm}}-i \bar{\theta}^{ \pm} \partial_{ \pm} \\
& \bar{D}_{ \pm}=-\frac{\partial}{\partial \bar{\theta}^{ \pm}}+i \theta^{ \pm} \partial_{ \pm}
\end{aligned}
$$

We wish to preserve as symmetries those transformations for which $\epsilon_{+}=\epsilon_{-}$. Define:

$$
\begin{aligned}
& \epsilon=\frac{1}{\sqrt{2}}\left(\epsilon_{+}+\epsilon_{-}\right) \\
& \tilde{\epsilon}=\frac{1}{\sqrt{2}}\left(\epsilon_{+}-\epsilon_{-}\right)
\end{aligned}
$$

and set $\tilde{\epsilon}=0$. Then

$$
\delta \Phi=\left(\epsilon \frac{Q_{-}-Q_{+}}{\sqrt{2}}+\bar{\epsilon} \frac{Q_{+}^{\dagger}-Q_{-}^{\dagger}}{\sqrt{2}}\right) \Phi
$$

We can define

$$
\theta=\frac{\theta^{+}-\theta^{-}}{\sqrt{2}}
$$

and

$$
Q=\frac{Q_{+}-Q_{-}}{\sqrt{2}}=\frac{\partial}{\partial \theta}+i \bar{\theta} \partial_{0} \quad Q^{\dagger}=\frac{Q_{+}^{\dagger}-Q_{-}^{\dagger}}{\sqrt{2}}=-\frac{\partial}{\partial \bar{\theta}}-i \theta \partial_{0}^{\dagger}
$$

so that

$$
\delta \Phi=\left(\epsilon Q-\bar{\epsilon} Q^{\dagger}\right) \Phi
$$


Similarly, we can define superspace derivatives:

$$
\begin{aligned}
& D=\frac{D_{+}-D_{-}}{\sqrt{2}}=\frac{\partial}{\partial \theta}-i \bar{\theta} \partial_{0} \\
& \bar{D}=\frac{\bar{D}_{+}-\bar{D}_{-}}{\sqrt{2}}=-\frac{\partial}{\partial \bar{\theta}}+i \theta \partial_{0} .
\end{aligned}
$$

\section{Appendix C. The tensor formula}

Here we state a formula for the tensor product of two sheaves. The support of this product sheaf is the intersection of the supports of the original sheaves.

Take a complex

$$
0 \rightarrow V_{0} \stackrel{d_{1}}{\longrightarrow} V_{1} \stackrel{d_{2}}{\longrightarrow} \cdots \stackrel{d_{m}}{\longrightarrow} V_{m} \rightarrow 0
$$

whose cohomology is a sheaf $E$, and a complex

$$
0 \rightarrow W_{0} \stackrel{\tilde{d}_{1}}{\longrightarrow} W_{1} \stackrel{\tilde{d}_{2}}{\longrightarrow} \cdots \stackrel{\tilde{d}_{n}}{\longrightarrow} W_{n} \rightarrow 0
$$

whose cohomology is a sheaf $F$. Then a complex whose cohomology is $E \otimes F$ is:

$$
0 \rightarrow V_{0} \otimes W_{0} \stackrel{D_{1}}{\longrightarrow} V_{0} \otimes W_{1} \oplus V_{1} \otimes W_{0} \stackrel{D_{2}}{\longrightarrow} V_{0} \otimes W_{2} \oplus V_{1} \otimes W_{1} \oplus V_{2} \otimes W_{0} \stackrel{d_{3}}{\longrightarrow} \cdots \rightarrow 0,
$$

where

$$
D_{r+s+1}(a \otimes b)=d_{r+1}(a) \otimes b+(-1)^{r} a \otimes \tilde{d}_{s+1}(b)
$$

for $a \in V_{r}$ and $b \in W_{s}$.

This formula is well known to mathematicians, to whom, however, the minus signs in (C.4) seem mysterious (c.f. p. 431 of [231]). Rather than proving this directly as a mathematical statement, we can give a physical interpretation. Consider two sets of fiber fields $\beta, \wp$ and $\tilde{\beta}, \tilde{\wp}$ which transform under two independent boundary symmetries $U(1)_{1,2}$. The $\beta$, correct deformed chiral constraints to have cohomology equal to $E$. That is

$$
\begin{aligned}
\left\{Q^{\dagger}, \beta_{2 n}\right\} & =d_{2 n-1} \wp_{2 n-1}^{\dagger} \\
{\left[Q^{\dagger}, \wp_{2 n+1}^{\dagger}\right] } & =d_{2 n} \beta_{2 n} .
\end{aligned}
$$

Similarly, $\tilde{\beta}, \tilde{\wp}$ satisfy the contraints:

$$
\begin{aligned}
\left\{Q^{\dagger}, \tilde{\beta}_{2 n}\right\} & =\tilde{d}_{2 n-1} \tilde{\wp}_{2 n-1}^{\dagger} \\
{\left[Q^{\dagger}, \tilde{\wp}_{2 n+1}^{\dagger}\right] } & =\tilde{d}_{2 n} \tilde{\beta}_{2 n}
\end{aligned}
$$


in order to describe $F$. We can combine $\beta_{k}, \wp_{k}^{\dagger}$ into an object $\gamma_{m}$ which is even(odd)

when $m$ is odd(even). If the left endpoint lives in $E \otimes F$, the fields for both $E$ and

$F$ should be excited. Thus we perform two boundary charge projections, each onto

charge sector +1 .

To see that this is equivalent to the complex (C.3) with exterior derivative $D$, examine variables invariant under the combination $U(1)_{1}-U(1)_{2}$. The gauge invariant combinations which satisfy deformed chiral constraints off shell are $\beta \tilde{\beta}, \beta \tilde{\wp}^{\dagger}, \wp^{\dagger} \tilde{\beta}, \wp^{\dagger} \tilde{\wp}^{\dagger}$. These combinations all have charge +1 under the remaining symmetry $\frac{1}{2}\left(U(1)_{1}+U(1)_{2}\right)$, and satisfy:

$$
\left[Q^{\dagger}, \gamma_{m} \tilde{\gamma}_{n}\right]=d_{m-1}\left(\gamma_{m-1}\right) \tilde{\gamma}_{n}+(-1)^{m-1} \gamma_{m} \tilde{d}_{n-1}\left(\tilde{\gamma}_{n-1}\right)
$$

Thus, the singlets of the confined gauge group $U(1)_{1}-U(1)_{2}$ transform under supersymmetry as fibers in the complex defining $E \otimes F$. 


\section{Chapter 10: Linear Sigma Model Toolshed for D-brane Physics}

Building on earlier work [232], we construct linear sigma models for strings on curved spaces in the presence of branes. Our models include an extremely general class of brane-worldvolume gauge field configurations. We explain in an accessible manner the mathematical ideas which suggest appropriate worldsheet interactions for generating a given open string background. This construction provides an explanation for the appearance of the derived category in D-brane physics complementary to that of [233].

\section{Introduction}

Main Entry: de $\cdot$ noue $\cdot$ ment

Function: noun

1. the final outcome of the main dramatic complication in a literary work

2. the resolution of a complex sequence of events

The study of supersymmetric D-branes in curved spaces is a dual-purpose endeavor. On one hand, these objects provide new probes of the stringy physics which was uncovered principally by the application of mirror symmetry. On the other hand, when coupled with orientifolds, they provide a large, relatively uncharted class of quasi-realistic string vacua.

Thus far, only a small part of the spectrum of branes on Calabi-Yau (CY) manifolds has been studied. While a classification of these objects, even in the geometrical limit, is lacking, an avenue toward systematic exploration is provided by the fact that all bundles have a description in terms of simpler components. Such a description, called a "resolution" (see [218] or [234] Chapter II, Corollary 5.18 ) is a sequence of maps between sums of rank-one bundles which encodes the

The material in this chapter appeared in "Linear sigma model toolshed for D-brane physics" with Simeon Hellerman [232] and is reprinted with permission of JHEP. 
non-triviality of the bundle of interest. As a means of clearly laying out the space of D-brane states, these sequences appear promising [233]. It should be possible to use this sequence data to make a useful model of the stringy physics.

A tractable description of the conformal field theory (CFT) describing the string dynamics on a CY is lacking (for any branes, or even in their absence) except for special values of the closed string moduli. A useful model of much of the physics at all points in moduli space is provided by linear sigma models (LSM's)[186]. Such models are two-dimensional quantum field theories which approach the desired CFT at long distances. Linear sigma models are in many cases the only available tools for extracting information about string backgrounds in the small-volume region, including the persistence of such backgrounds beyond perturbation theory. The LSM is the ideal framework to

\section{HARNESS THE AWESOME POWER OF HOMOLOGICAL ALGEBRA.}

Already the shortest such sequences ("monads") have appeared in linear sigma model constructions, first for heterotic strings [186,235], and more recently for open strings [232].

In this chapter, we make linear models for bundles whose resolution has an arbitrary number of nodes ${ }^{42}$. More generally, the models we make generate any bundle which can be realized as the cohomology of a sequence of sums of line bundles.

The sequences which encode the data for these models are the same ones that appear as building-blocks for the derived category of [233]. ${ }^{43}$ As in other incarnations of open string field theory [239], the condensation of spacetime tachyons is manifested on the string worldsheet as flow to the infrared. In our construction, the equivalence of quasi-isomorphic complexes is a consequence of universality in the sense of the renormalization group.

There a second way in which our work relates to [233] which we explain in the concluding section.

In addition to any motivation from recent exciting ideas about abstract descriptions of D-brane spectra, our construction would be needed for an attempt to make contact with phenomenology through this type of four-dimensional $\mathcal{N}=1$

42 It was suggested recently [233] that this would be useful.

43 Early work in this direction includes [236,237,238]. 
string vacuum. Certainly any systematic exploration of this class of vacua would incorporate the more general models we construct.

The plan of the chapter is as follows. In $\S 2$ we (p)review the construction [232] of a linear sigma model coupling open strings to B-type branes, and more specifically to a bundle defined by a one-step sequence. In $\S 3$ we explain why this construction fails for more general bundles whose resolutions do not terminate after one step, and explain our solution. In $\S 4$ we discuss a linear model for an even broader class of bundles, namely those which are not pullbacks of bundles on the ambient space in which the CY is embedded. In $\S 5$ we explore the relation of our linear models to possible linear models for heterotic strings whose left-moving fermions couple to these more general bundles. We close with a discussion of applications and the extension of this set of ideas to CY's and branes which are not complete intersections. In appendix A we give more details about our models. In appendix $\mathrm{B}$ we describe another formulation of our models, of which the one in the body of the chapter is a gauge-fixed version.

Other work on linear models for open strings includes [240,241,242,243,244].

\section{Brief review of the construction for one step}

We present this discussion in the context of a CY hypersurface in projective space, but the generalization to any complete intersection in a toric variety should be clear. The simplest example of the monad construction defines a holomorphic vector bundle, $V$, from the sequence of maps

$$
0 \rightarrow V \rightarrow E_{1} \equiv \bigoplus_{a_{1}} \mathcal{O}\left(n_{a_{1}}\right) \stackrel{d_{1}}{\longrightarrow} E_{2} \equiv \bigoplus_{a_{2}} \mathcal{O}\left(n_{a_{2}}\right) \rightarrow 0
$$

The first map is inclusion and the second map is

$$
\begin{aligned}
d_{1}: E_{1} & \rightarrow E_{2} \\
\beta^{a_{1}} & \mapsto \sum d_{1 a_{1}}^{a_{2}}(\phi) \beta^{a_{1}} .
\end{aligned}
$$

This sequence is exact in the sense that the kernel of one map is the image of the previous one. For our purposes, the bundles $E_{l}$ are sums of powers of the hyperplane bundle over a projective space. We want to consider type II string theory on a Calabi-Yau hypersurface, $X$, in the projective space. A linear sigma 
model which realizes open strings ending on a brane wrapping $X$ with gauge bundle $V$ works as follows [232].

The bulk fields are the same as in the $(2,2)$ linear model for $X$. For ease of exposition we present the model for a hypersurface in $\mathrm{P}^{4}$ defined by a homogeneous polynomial $G$ of degree $d=5$. Then one has a single $U(1)$ gauge multiplet under which the chiral multiplets $\Phi^{i}, i=1 \ldots 5$ carry charge 1 and $P$ carries charge -5 . There is a $(2,2)$ superpotential $W_{b u l k}=P G(\Phi)$. In accordance with the notation of the relevant (B-type) topological field theory, we refer to the fermions in a bulk chiral multiplet as

$$
\Theta=\frac{1}{\sqrt{2}}\left(\psi_{+}-\psi_{-}\right), \quad \eta=\frac{1}{\sqrt{2}}\left(\psi_{+}+\psi_{-}\right)
$$

We refer to [186] for more details.

We give these fields couplings to boundary matter which respect B-type supersymmetry. By B-type supersymmetry we mean a subalgebra of the bulk $(2,2)$ supersymmetry generated by a linear combination of $Q_{+}$and $Q_{-}$rather than a linear combination of $Q_{+}$and $Q_{-}^{\dagger}$. Boundary conditions preserving such a subalgebra are associated with branes of even codimension in the CY [245]. We call the conserved supercharges $Q$ and $Q^{\dagger}$. The representation theory of their algebra is similar to that of $(0,2)$ two-dimensional supersymmetry and is worked out in [232]. The two kinds of multiplets we will need are: Fermi multiplets, $\beta$, have a fermion as their lowest component, and chiral multiplets, $\wp$, have a boson as their lowest component. Both satisfy either the chiral constraint, $Q^{\dagger}(\beta, \wp)=0$, or some deformation thereof. The consistency conditions on such deformations will play a key role in building the more general bundles.

\subsection{About kinetic terms}

A Fermi multiplet contains a complex fermion, $\beta$, and an auxiliary complex boson, $b$. A supersymmetric kinetic term is 44

$$
\int d^{2} \theta \beta^{\dagger} \beta=b^{\dagger} b-i\left(\nabla_{0} \beta^{\dagger}\right) \beta+i \beta^{\dagger}\left(\nabla_{0} \beta\right)
$$

\footnotetext{
44 We use gauge-covariantized supercharges, so we omit the usual $e^{q V}$ 's.
} 
where

$$
\nabla_{0} \equiv \frac{i}{2}\left\{Q, Q^{\dagger}\right\}
$$

A boundary chiral multiplet contains a complex boson, $\wp$, and a fermion $\xi$. In addition to a usual kinetic term of the form

$$
\int d^{2} \theta \wp^{\dagger} \nabla_{0} \wp
$$

we add a magnetic field term:

$$
\int d^{2} \theta B \wp^{\dagger} \wp=B \xi^{\dagger} \xi+B \wp^{\dagger} \nabla_{0} \wp-\left(\nabla_{0} B \wp^{\dagger}\right) \wp
$$

where $B$ is a constant. Consider a limit where $B$ is big so that we can ignore the kinetic terms for $\wp$, c.f. e.g. [246]. This approximation can be justified by the fact that this term is less irrelevant than the kinetic one. In that case, the momentum conjugate to $\wp$ is $i B \wp^{\dagger}$. So canonical quantization gives

$$
\left[\wp, \wp^{\dagger}\right]=1 / B
$$

The coupling to the magnetic field masses up the fermion $\xi$ and it halves the number of $\wp$ degrees of freedom - i.e. the set of states made by $\wp$ is now just the Hilbert space of a harmonic oscillator. It makes the $\wp$ multiplet into an exact bosonic analog of a Fermi multiplet. Since we work in an approximation where the magnetic field term dominates the kinetic term, we will drop the usual kinetic term altogether and adopt a normalization for $\wp$ such that $B \equiv 1$.

\subsection{The model for a monad}

On the boundary of the string, introduce Fermi multiplets, $\beta^{a_{1}}$, which live in the bundle $E_{1}$, and chiral multiplets, $\wp_{a_{2}}$, which live in the bundle $E_{2}^{\star}$. In this case the half-superspace integrand which generates the bundle $V$ is

$$
W=\wp_{a_{2}} d_{1 a_{1}}^{a_{2}} \beta^{a_{1}}
$$

which we abbreviate as

$$
W=\wp d_{1} \beta
$$

(We will suppress indices whenever possible!) 
After integrating out the auxiliary bosons in $\beta_{a}$, this leads to the following interactions on the boundary

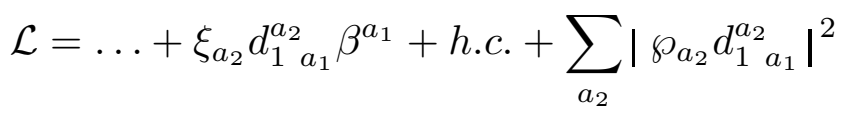

The finishing touch we need to put on the monad model is to implement a charge projection on the boundary which guarantees the right number of ChanPaton (CP) states - i.e., that only one Chan-Paton fermion at a time will be excited, no more and no less. Specifically, we gauge the symmetry which acts only on boundary fields, as

$$
\begin{aligned}
& \wp \mapsto e^{i \gamma} \wp \\
& \beta \mapsto e^{-i \gamma} \beta .
\end{aligned}
$$

This is done by adding a supersymmetry singlet, one-dimensional gauge field $a_{0}$ on the boundary, which couples as

$$
\mathcal{L}_{a}=\int_{\partial \Sigma} a_{0}\left(j_{S}-1\right)
$$

where $j_{S}$ is the boundary symmetry charge. Since each excitation of the $\beta$ fermions is in effect a 'quark' at the left endpoint of the open string (or an 'antiquark' at the right endpoint), the charge projection restricts the system to a subsector of the Hilbert space where the open string worldsheet couples to the gauge field as a section of $V \otimes V^{*}[232]$.

\section{Any resolution}

Consider a bundle $V$ over a Calabi-Yau manifold which is a hypersurface in projective space. Such a bundle has a resolution of the form

$$
0 \rightarrow V \rightarrow E_{1} \stackrel{d_{1}}{\longrightarrow} E_{2} \stackrel{d_{2}}{\longrightarrow} E_{3} \rightarrow \cdots
$$

which is an exact sequence and the $E_{l}$ s are direct sums of line bundles. The map from $E_{l}$ to $E_{l+1}$ depends on $\phi$ and we will call it $d_{l}$. Let $k_{l}$ be the rank of $E_{l}$.

At this point, the important point to make is what goes wrong with the naive model when $d_{1}$ has a cokernel. This is twofold: firstly, there are extra massless $\wp$ s on the boundary, each of which gives an energetically degenerate harmonic-oscillator 
spectrum of states, leading to the wrong spectrum of CP factors. Secondly, and perhaps more importantly, a generic deformation of the matrix elements of $d_{1}$ will destroy the brane. This happens because the matrix $d_{1}$ imposes overconstrained conditions on $\beta$, which are inconsistent rather than redundant for a generic deformation. So under a random deformation, the equations of motion for the $\wp \mathrm{s}$ and $\beta \mathrm{s}$ will simply set them to zero at low energies, meaning that no states will satisfy the boundary charge projection - there will be no CP factors. This is connected with the problem of massless $\wp$ 's in that the superpartners of the $\wp$ 's are the Goldstone fermions for the spontaneous breaking of supersymmetry.

Now for a model with the correct behavior. The sections of the line bundles which we introduce will be alternately Fermi multiplets, $\beta_{(l)}^{a}$, and chiral multiplets, $\wp_{a(l+1)}$. They are Fermi multiplets at the first step, as in the monad case, because it is states of massless fermions that play the role of the CP factors (or the left-moving current algebra in a $(0,2)$ theory). The basic idea is that the fermions at the third step pair up with the "extra" massless fermion partners of the $\wp$ 's at the second step (which arise because $d_{1}$ has a cokernel). If the sequence does not terminate there, the partners of the bosons at the fourth step pair up with the extra fermions at the third step, and this process of lifting kernels and images continues until the sequence terminates.

In the general case the boundary symmetry acts on all chiral multiplets with one phase and all Fermi multiplets with the opposite phase.

For ease of discussion, we give the details for a bundle with a two-step resolution:

$$
0 \rightarrow V \rightarrow E_{1} \stackrel{d_{1}}{\longrightarrow} E_{2} \stackrel{d_{2}}{\longrightarrow} E_{3} \rightarrow 0
$$

We will also assume for simplicity that the line bundles making up $E_{i}$ at each step are the same, i.e.,

$$
E_{i} \equiv \mathcal{O}\left(n_{i}\right)^{\oplus k_{i}}
$$

This assumption is in no way essential to the construction.

Then the degrees $\Delta_{1,2}$ of the polynomials defining the maps $d_{1,2}$ are $n_{2}-n_{1}$ and $n_{3}-n_{2}$, respectively. Here is the field content:

- Bulk fields: the usual $(2,2)$ multiplets $\left(\phi, P_{b u l k}, \sigma\right)$ where $P_{b u l k}$ is the bulk $P$-multiplet. 
- A set of boundary fermi multiplets $\beta^{i}$, a smoothly varying subspace of which will define the desired bundle. They satisfy the usual chiral constraint $\left\{Q^{\dagger}, \beta\right\}=0$ and their gauge charge is $n \equiv n_{1}$.

- A set of boundary chiral multiplets $\wp_{i}$ with gauge charges $-n_{2}=-\left(n+\Delta_{1}\right)$. Instead of the usual chiral constraint $\left[Q^{\dagger}, \wp\right]=0$ these will obey a deformed chiral constraint, which will fix the $Q^{\dagger}$ variation of $\wp$ in terms of the other fields of the problem.

- A set of boundary multiplets $\beta^{\prime}$ which boundary-symmetry charge +1 and gauge charge $n_{3} \equiv n+\Delta_{1}+\Delta_{2}$. They will turn out to be forced to satisfy the opposite of the chiral constraint: $\left\{Q, \beta^{\prime}\right\}=0$.

Now for the interactions. The half-superspace integral will be:

$$
\int d \theta W \equiv \int d \theta \wp d_{1} \beta
$$

We can add the obvious gauge-invariant kinetic term for the $\beta, \beta^{\prime}$ multiplets:

$$
\int d^{2} \theta \cdot \beta^{\dagger} \beta+\beta^{\prime \dagger} \beta^{\prime}
$$

The key to obtaining the correct physics is the multiplet structure of $\wp$ :

Since $d_{1}$ has a cokernel, the condition for the superpotential $W$ to be annihilated by $Q^{\dagger}$ does not require $Q^{\dagger}$ to annihilate $\wp$. Indeed, $\left[Q^{\dagger}, \wp\right]=($ anything $) \cdot d_{2}$ will suffice.

There is an obvious choice: $\left[Q^{\dagger}, \wp\right]=i \beta^{\prime \dagger} d_{2}$. Since for a two-step sequence $d_{2}$ is onto, the nilpotence of $Q^{\dagger}$ forces $\left\{Q^{\dagger}, \beta^{\prime \dagger}\right\}=0$ - so $\beta^{\prime}$ satisfies the opposite of the usual chiral constraint.

This constraint completely determines the supersymmetry transformations of the new multiplet. This is one of the two key ingredients in this new type of model. The second is the observation that for a boson living on the boundary, it is consistent to add to the Lagrangian a magnetic field coupling for the complex target space fiber coordinates:

$$
\int d^{2} \theta \wp \wp^{\dagger}
$$

In fact it is necessary to add this term to obtain the mass terms and Yukawa couplings that give the desired physics in the infrared. 
Rather than listing all the terms in the action and the supersymmetry transformations here, we leave that to the Appendix. In this section we will discuss only the terms which will be important for causing the model to flow to the correct brane configuration at low energies, in the 'large radius' phase of the worldsheet theory.

To clarify the content of the low energy theory we begin by integrating out the auxiliary fields $b$ in the fermi multiplets and also the superpartners $\xi$ of $\wp$, which become auxiliary at low energies in the presence of the large magnetic field. With auxiliary fields eliminated, the key terms in the Lagrangian are then:

- Derivative terms for the physical bosons and fermions of the system:

$$
i \cdot\left[\beta^{\dagger}\left(\nabla_{0} \beta\right)+\left(\nabla_{0} \wp\right) \wp^{\dagger}+\beta^{\prime \dagger}\left(\nabla_{0} \beta^{\prime}\right)-\text { h.c. }\right]
$$

For the fermions these are just standard kinetic terms; for the bosons the standard kinetic term is irrelevant and their dynamics at this scale is dominated by lowest Landau level physics; the number of physical degrees of freedom is effectively reduced by a factor of two and the complex bosonic fiber becomes a product of noncommutative $\mathbb{C}^{1}$ 's. Now the boson $\wp$ really does have similar kinematics to the fermions $\beta$ and $\beta^{\prime}$, except with opposite statistics, i.e., $\left[\wp_{i}, \wp_{j}^{\dagger}\right]=\delta_{i j}$.

- Mass terms for the fermions:

$$
\beta^{\dagger}\left(d_{1}^{\dagger} d_{1}\right) \beta+\beta^{\prime \dagger}\left(d_{2} d_{2}^{\dagger}\right) \beta^{\prime}
$$

- Mass terms for the bosons:

$$
\wp\left(d_{1} d_{1}^{\dagger}+d_{2}^{\dagger} d_{2}\right) \wp^{\dagger}
$$

Note that because of the large magnetic field, the physical mass of the bosons is proportional to the coefficient of $|\wp|^{2}$ rather than to the square root of that coefficient.

In the next subsection we explain why these potentials and Yukawa couplings are preicisely what we need to yield the correct sigma model at low energies. 
Interestingly, the supersymmetry transformations take a very nice form with $\xi$ and the other auxiliary fields integrated out:

$$
\begin{array}{rr}
\{Q, \beta\}=-i d_{1}^{\dagger} \wp^{\dagger} & \left\{Q, \beta^{\dagger}\right\}=0 \\
\left\{Q^{\dagger}, \beta\right\}=0 & \left\{Q^{\dagger}, \beta^{\dagger}\right\}=+i \wp d_{1} \\
{\left[Q, \wp^{\dagger}\right]=+i d_{2}^{\dagger} \beta^{\prime}} & {[Q, \wp]=-i \beta^{\dagger} d_{1}^{\dagger}} \\
{\left[Q^{\dagger}, \wp^{\dagger}\right]=+i d_{1} \beta} & {\left[Q^{\dagger}, \wp\right]=-i \beta^{\prime \dagger} d_{2}} \\
\left\{Q, \beta^{\prime}\right\}=0 & \left\{Q, \beta^{\prime \dagger}\right\}=-i \wp d_{2}^{\dagger} \\
\left\{Q^{\dagger}, \beta^{\prime}\right\}=+i d_{2} \wp^{\dagger} & \left\{Q^{\dagger}, \beta^{\prime \dagger}\right\}=0
\end{array}
$$

We note again that these transformations are consistent with the supersymmetry algebra (in particular, $Q^{2}=0$ ) because $d_{2} \circ d_{1}=0$. The relation $\left\{Q, Q^{\dagger}\right\}=$ $2 H$ holds on-shell, that is when the fields satisfy their (first-order) equations of motion. Although the limit in which the two-derivative kinetic term for the bosons vanishes simplifies the algebra greatly, the model is consistent without using this approximation.

The generalization to sequences of an arbitrary number of steps should be clear. The $Q^{\dagger}$-variation of a field associated with a given node encodes the previous map, while the $Q$-variation encodes the next map. So, for example, to make a 3 -step sequence, we would add some $\wp^{\prime}$ fields satisfying the undeformed chiral constraint $\left[Q^{\dagger}, \wp^{\prime}\right]=0$ and deform the constraint on $\beta^{\prime}$ to $\left\{Q, \beta^{\prime}\right\}=d_{3}^{\dagger} \wp^{\prime \dagger}$. The model works for a sequence of arbitrary length; one never needs to add another superpotential term, only deform the chiral constraint by hand at each step according to the data of the sequence and add the appropriate full-superspace terms. In the appendix we write down the model for a sequence of arbitrary length.

\subsection{Large-radius analysis}

The point of this section is to prove that we get the right low-energy behavior for the two-step model from the interactions discussed in the previous subsection. Make the bulk FI coefficient $r$ large and positive, so that we are in the large-radius CY phase of the bulk theory. 
Let us determine the supersymmetric vacuum of the theory by setting to zero the supersymmetry variations in equation (3.6):

$$
\begin{aligned}
& 0=d_{1} \beta \\
& 0=\wp d_{1} \\
& 0=\wp d_{2}^{\dagger} \\
& 0=d_{2}^{\dagger} \beta^{\prime}
\end{aligned}
$$

The first equation tells us that the massless $\beta$ s live in the kernel of $d_{1}$. Since $d_{2}$ is surjective, the last equation tells us that $\beta^{\prime}$ must vanish. The two middle equations tell us that $\wp$ is closed and co-closed which, since the sequence is exact at $E_{2}$, tells us that $\wp$ must vanish as well. This is the desired physics.

We would arrive at the same conclusion by a direct examination of the Lagrangian, without making use of supersymmetry. The statement that the sequence we examine has no cohomology at the middle node is the statement that there is no nonzero fiber annihilated both by $d_{2}$ and by $d_{1}^{\dagger}$. As a result, $d_{2}^{\dagger} d_{2}+d_{1} d_{1}^{\dagger}$ is an invertible matrix, so all components of $\wp$ are set to zero at low energies by their equations of motion. Similar reasoning shows that all $\beta^{\prime}$ are set to zero by their mass terms at low energies, and that the surviving subset of the $\beta$ 's is the kernel of $d_{1}$, just as we wanted.

Note that this analysis makes it clear that there is a direct relation between cohomology of the sequence and massless worldsheet fields. In particular, if under a deformation the defining polynomials the sequence fails to be exact at some point, $\phi_{\star}$, in the CY, closed and co-closed will no longer imply zero and we will find massless $\beta^{\prime}$ s and $\wp$ 's.

In summary, as in the simpler case, given a non-degeneracy condition for the sequence (analogous to $G=d_{1}=0$ has no solutions in the monad case [235]) all of the $\wp$ 's vanish in vacuum, and the mass matrix for the fermions imposes the sequence.

\subsection{Examples}

The first example we study is a very trivial one, namely a multi-step resolution for a twobrane on a two-torus with a trivial line bundle. Make the $T^{2}$ as a cubic hypersurface in $\mathbb{P}^{2}$. Consider the Koszul complex over the ambient $\mathbb{P}^{2}$,

$$
0 \rightarrow V \stackrel{i}{\hookrightarrow} \mathcal{O}(1)^{\oplus 3} \stackrel{d_{1}}{\longrightarrow} \mathcal{O}(2)^{\oplus 3} \stackrel{d_{2}}{\longrightarrow} \mathcal{O}(3) \rightarrow 0
$$


where the maps are

$$
d_{1}=\left(\begin{array}{ccc}
0 & \phi_{2} & -\phi_{1} \\
-\phi_{2} & 0 & \phi_{0} \\
\phi_{1} & -\phi_{0} & 0
\end{array}\right) \quad \text { and } \quad d_{2}=\left(\phi_{0}, \phi_{1}, \phi_{2}\right)
$$

the inclusion is induced by

$$
i=\left(\begin{array}{c}
\phi_{0} \\
\phi_{1} \\
\phi_{2}
\end{array}\right)
$$

One can see by computing its Chern classes that the line bundle $V$ defined by this sequence is in fact trivial ${ }^{45}$.

To model a string coupling to this brane, we add on its boundary three fermi fields $\beta$ of charge 1 , three chiral fields $\wp$ of charge -2 , and another fermi field $\beta^{\prime}$ of charge 3 . We add the superpotential

$$
W=\wp d_{1} \beta=\epsilon_{i j k} \beta^{i} \wp^{j} \phi^{k}
$$

and implement the chiral constraints and charge projections discussed above.

Obviously this brane could also be constructed by simply adding a neutral fermion on the end of the string. To see the relation to the above model, define an effective neutral fermion, $\gamma$, by

$$
\beta^{i}=\phi^{i} \gamma+\text { massive }
$$

Setting to zero $\wp, \beta^{\prime}$ and the massive components of $\beta$ then solves the vacuum equations identically. So the multistep model does flow in the IR to the theory of a single neutral fermion.

For a less trivial example, we study the pullback to the $\mathrm{CY}$ of the tensor square $V \equiv \mathrm{T}^{*} \mathbb{P}^{n} \otimes \mathrm{T}^{*} \mathbb{P}^{n}$ of the cotangent bundle of $\mathbb{P}^{n}$. This is defined by the sequence

$$
0 \rightarrow V \stackrel{i}{\hookrightarrow} \mathcal{O}(-2)^{\oplus(n+1)^{2}} \stackrel{d_{1}}{\longrightarrow} \mathcal{O}(-1)^{\oplus 2 n+2} \stackrel{d_{2}}{\longrightarrow} \mathcal{O}(0) \rightarrow 0
$$

where the maps are

$$
d_{1}: \beta_{i j} \mapsto\left(\phi^{j} \beta_{i j}, \phi^{i} \beta_{i j}\right) \quad \text { and } \quad d_{2}:(\wp, \hat{\wp}) \mapsto \phi^{i}\left(\wp_{i}-\hat{\wp}_{i}\right)
$$

\footnotetext{
45 This statement is false. The example of the CY 1-fold is special in that the target space is not simply connected; however the existence of a global section (demonstrated below) means that the bundle is in fact trivial.
} 
To build this bundle, we add $(n+1)^{2}$ fermi multiplets $\beta_{i j}$ of charge $-2,2 n+2$ chiral multiplets $\wp_{i}$ and $\hat{\wp}_{i}$ of charge -1 , and one neutral fermi multiplet $\beta^{\prime}$. The superpotential is

$$
W=\wp_{i} \phi^{j} \beta_{i j}+\hat{\wp}_{j} \phi^{i} \beta_{i j}
$$

and the nontrivial deformed constraints are

$$
\begin{array}{r}
{\left[Q, \wp_{i}\right]=-i \beta_{i j}^{\dagger} \phi^{j \dagger} \quad\left[Q, \hat{\wp}_{j}\right]=-i \beta_{i j}^{\dagger} \phi^{i \dagger}} \\
{\left[Q^{\dagger}, \wp_{i}\right]=-i \beta^{\prime \dagger} \phi^{i} \quad\left[Q^{\dagger}, \hat{\wp}_{i}\right]=i \beta^{\prime \dagger} \phi^{i}} \\
\left\{Q, \beta^{\prime \dagger}\right\}=-i(\wp-\hat{\wp})^{\dagger} \phi^{i \dagger} .
\end{array}
$$

We have given two examples of smooth bundles with constant fiber dimension using resolutions of finite length. It would be nice to understand more about the physics of the sheaves defined by more general choices of ranks and charges in a multistep sequence (3.1).

\section{Bundles which do not extend to the ambient space}

In fact we lose some generality by considering only pullbacks of bundles on the ambient toric variety. Bundles which extend generate in general a sublattice of finite index in the lattice of all $K$-theory classes of bundles on the target variety.

In order to see how to generate linear models for more general bundles, let us consider the case of a hypersurface defined by $G(\phi)=0$. The bulk then has a single $P$-field and a superpotential $W_{\text {bulk }} \equiv P G(\phi)$. Non-extending bundles can be realized as the cohomology of a sequence over the coordinate ring of the variety, i.e. the cohomology of a set of maps $d_{n}$ such that $d_{n+1} d_{n}=M_{n+1 \mid n} G(\phi)$ for some matrix $M_{n+1 \mid n}$ of holomorphic polynomials.

The key fact in the following construction is that

$$
d_{n+2} M_{n+1 \mid n}=M_{n+2 \mid n+1} d_{n}
$$

Proof: $G(\phi)\left(d_{n+2} M_{n+1 \mid n}-M_{n+2 \mid n+1} d_{n}\right)=\left(d_{n+2} d_{n+1}\right) d_{n}-d_{n+2}\left(d_{n+1} d_{n}\right)=0$ by associativity. Since $G$ is nonvanishing and polynomial rings contain no zero divisors, that means the second factor $d_{n+2} M_{n+1 \mid n}-M_{n+2 \mid n+1} d_{n}$ must vanish and the statement follows. 
We now set the bulk $\eta$ multiplet on shell; in particular this means $F^{\dagger^{P}} \equiv$ $\left\{Q^{\dagger}, \eta^{\dagger^{P}}\right\}=G(\phi)$. For a two-step resolution we take the boundary superpotential to be

$$
W=\left(\wp d_{1}-\eta^{\dagger} \beta^{\prime \dagger} M_{2 \mid 1}\right) \beta
$$

and the (deformed) chiral constraints to be

$$
\left\{Q^{\dagger}, \beta\right\}=0 \quad\left[Q^{\dagger}, \wp\right]=\beta^{\prime \dagger} d_{2} \quad\left\{Q^{\dagger}, \beta^{\prime}\right\}=i d_{2} \wp^{\dagger}
$$

Clearly the supersymmetry algebra closes and the superpotential is annihilated by $Q^{\dagger}$

The extension to a multistep sequence over a hypersurface is straightforward. For three steps, for instance, the superpotential is the same, and the supersymmetry transformations are

$$
\begin{aligned}
\left\{Q^{\dagger}, \beta\right\} & =0 \\
{\left[Q^{\dagger}, \wp\right] } & =\beta^{\prime \dagger} d_{2}-\eta^{\dagger} \wp^{\prime} M_{3 \mid 2} \\
\left\{Q^{\dagger}, \beta^{\prime}\right\} & =i d_{2} \wp^{\dagger} \quad\left\{Q^{\dagger}, \beta^{\prime \dagger}\right\}=\wp^{\prime} d_{3} \\
{\left[Q^{\dagger}, \wp^{\prime}\right] } & =0
\end{aligned}
$$

For an arbitrary number of steps, take the same superpotential and use the constraints

$$
\begin{aligned}
\left\{Q^{\dagger}, \beta\right\} & =0 \\
{\left[Q^{\dagger}, \wp\right] } & =-i \beta^{\prime \dagger} d_{2}-\eta^{\dagger} \wp^{\prime} M_{3 \mid 2} \\
\left\{Q^{\dagger}, \beta^{\prime \dagger}\right\} & =i \wp^{\prime} d_{3}-\eta^{\dagger}{ }^{P} \beta^{\prime \prime \dagger} M_{4 \mid 3} \\
& \ldots
\end{aligned}
$$




\section{Discussion}

\subsection{Relation to heterotic models}

\begin{tabular}{|c|c|c|c|}
\multicolumn{1}{r}{ Field } & \multicolumn{1}{c}{$q_{G}$} & \multicolumn{1}{c}{$q_{L}$} & $q_{R}$ \\
\hline$\Phi_{i}$ & $w_{i}$ & 0 & $\frac{w_{i}}{m}$ \\
\hline$\Gamma$ & $-d$ & 0 & $1-\frac{d}{m}$ \\
\hline$\beta$ & $n_{1}$ & 1 & $\frac{n_{1}}{m}$ \\
\hline$\wp$ & $-n_{2}$ & -1 & 0 \\
\hline$\beta^{\prime}$ & $n_{3}$ & 1 & $1-\frac{d_{2}}{m}$ \\
\hline
\end{tabular}

One might hope that one could use similar technology to make linear models for heterotic strings coupling to these bundles. In fact, our spectrum of fields was motivated by the anomaly coefficients and cancellation conditions that one would have in the heterotic case. In the table at left we write the gauge charges, boundary symmetry charges, and R-symmetry charges of our Table 1: The gauge charges and "left-moving $U(1)$ " charges of the fields.

fields for a generic two-step model.

Now imagine that they are instead representations of $(0,2)$ supersymmetry, as in [235]. We call the boundary symmetry charge $q_{L}$ because in its heterotic incarnation, it is the charge under the left-moving $U(1)$ which becomes part of the spacetime gauge group (and a $Z_{2}$ subgroup of which provides one of the GSO projections). Using these charges we would calculate the anomaly in the left-moving $U(1)$ to be

$$
\mathcal{A}(L, G) \propto \sum_{\text {fields }} q_{G} q_{L}=k_{1} n_{1}-k_{2} n_{2}+k_{3} n_{3}
$$

This is just $c_{1}(V)^{46}$. Note that consistency only requires this to vanish mod 2 , since only a $Z_{2}$ subgroup of $U(1)_{L}$ is gauged. The gauge anomaly would be

$$
\mathcal{A}(G, G) \propto \sum_{\text {fields }}(-1)^{\text {fermi }} q_{G} q_{G} \propto c_{2}(V)-c_{2}(X)
$$

46 The chern classes of the bundle $V$ are determined by the sequence (3.1) to be

$$
c(V)=\prod_{l=0} c\left(E_{l}\right)^{(-1)^{l}}
$$

and in particular $c_{1}(V)=\sum_{l}(-1)^{l} \sum_{a_{l}} n_{a_{l}} J$, and $c_{2}(V)=\sum_{l} \sum_{a_{l} \neq a_{l}^{\prime}} n_{a_{l}} n_{a_{l}^{\prime}} J^{2}$ where $J$ is the $(1,1)$ form on the CY. 
The anomaly in the $U(1)_{R}$ symmetry would be (modulo the gauge anomaly)

$$
\mathcal{A}(R, G) \propto \sum_{\text {fields }} q_{G} q_{R}=\sum_{i} w_{i}-d,
$$

the first chern class of the hypersurface.

As in $[235,247]$ we can calculate the left-moving central charge of our model as an anomaly-matching coefficient in the massive theory. Specifically, the leftmoving central charge is the would-be (if it were gauged) quadratic anomaly of the left-moving $U(1)$ symmetry. This is $r=k_{0}-k_{1}+k_{2}$, the rank of the bundle, as expected.

However, while the spectrum seems to be correct, the interactions we would add to give the right vacuum structure do not respect two-dimensional Lorentz invariance. An equivalent way to phrase the obstruction is that the desired interactions break the $U(1)$ R-symmetry which is thought to flow to the R-symmetry of the right-moving $\mathcal{N}=2$ superconformal algebra. It is possible that a new R-symmetry appears in the IR, but without a UV candidate for the IR R-symmetry one loses some confidence that one has identified the correct LSM. This is puzzling, but we still hope to find a LSM for the multi-step resolution in the heterotic case. Given that nonperturbative conformal invariance of $(0,2)$ models has only been proven using the linear models [248], it would be fascinating if no heterotic LSM could be found for multi-step bundles.

We also hope to find an argument that motivates the field content directly in the open string case, such as a direct relation between the RR charge of the D-brane configuration and the spectrum of worldsheet fields.

\subsection{Other phases and singularities}

One of the great successes of the linear sigma model approach to CY physics is an automatic description of the stringy physics of small-volume phases of the theory. We are still working out the behaviour of the monad theories when the FI parameter is large and negative [232], and we leave the application of that analysis to these more intricate models for future work.

But there is a possibility of new physics from our multistep bundles. When the moduli of the bundle are deformed in such a way that the maps beyond the first step degenerate, cohomology will appear at higher nodes. It would be interesting 
to see if the resulting massless worldsheet fields have any special signatures in the spacetime physics.

We have been assuming thoughout this chapter that the sequence

$$
0 \rightarrow E_{1} \stackrel{d_{1}}{\longrightarrow} E_{2} \stackrel{d_{2}}{\longrightarrow} E_{3} \rightarrow \cdots
$$

was chosen so that its cohomology was a bundle. In fact, the cohomology of such a sequence will generically be a more general coherent sheaf (espoused by Harvey and Moore [249] as the proper mathematical characterization of wrapped D-branes). Work is in progress to harness this fact to study D-brane configurations without space-filling branes [232].

\subsection{Other classes of models}

In addition to the heterotic models discussed above, we are hoping to extend the construction to:

1. $(2,2)$ linear models for varieties which are not complete intersections. The generic CY manifold is such a beast. If a variety is not a complete intersection, it means that the number of defining equations is bigger than its codimension. As a result, there are relations among these equations, and in general relations among these relations... There is again a sequence of maps resolving the ideal of the variety. We have made some progress towards such $(2,2)$ models using extra gauge symmetries.

2. Open strings in the presence of branes wrapping submanifolds which are not complete intersections.

If we can accomplish item 1 above, our technology will be very useful for the program of [233]. In particular, the $4 \mathrm{~d} \mathcal{N}=1$ field theory which is proposed to describe the space of branes with fixed charge involves a superpotential with relations among the vacuum equations, and relations among these relations ... 


\section{Appendix A. Full supersymmetry transformations and lagrangian}

In this appendix we give a more detailed description of the supersymmetry structure of our models, and present the model for a sequence with an arbitrary number of nodes.

First we present the gauge-covariantized supersymmetry transformations for the two-step case and show that we get all of the terms we need in the action to get the desired massless degrees of freedom. (We do not write down terms in the bulk action which are given in [186].) We suppress indices on the fields.

The transformations of the bulk multiplets under the reduced superalgebra are:

$$
\begin{array}{rlrl}
{[Q, \phi]} & =-i \Theta & {\left[Q^{\dagger}, \phi\right]} & =0 \\
\{Q, \Theta\} & =0 & \left\{Q^{\dagger}, \Theta\right\}=2 \nabla_{0} \phi
\end{array}
$$

and

$$
\begin{array}{ll}
\{Q, \eta\}=F & \left\{Q^{\dagger}, \eta\right\}=2 \tilde{\nabla}_{1} \phi^{\dagger} \\
{[Q, F]=0} & {\left[Q^{\dagger}, F\right]=-2 i \nabla_{0} \eta+2 i \tilde{\nabla}_{1} \Theta+2 i q \phi\left(\lambda_{+}^{\dagger}+\lambda_{-}^{\dagger}\right) .}
\end{array}
$$

where $\tilde{\nabla}_{1} \equiv \frac{i}{2 \sqrt{2}}\left\{Q, Q_{+}^{\dagger}+Q_{-}^{\dagger}\right\}$, the anticommutator of $Q$ with the broken supercharge.

We will introduce the notation $\xi$ for the fermionic superpartner of $\wp$. So:

$$
\begin{gathered}
\left\{Q, \beta^{\prime \dagger}\right\}=b^{\prime} \\
\left\{Q^{\dagger}, \beta^{\prime \dagger}\right\}=0 \quad\left\{Q, \beta^{\prime}\right\}=0 \\
{\left[Q, b^{\prime}\right]=0} \\
{\left[Q^{\dagger}, b^{\prime}\right]=-2 i \nabla_{0} \beta^{\prime \dagger}} \\
{[Q, \wp]=-i \xi \quad\left[Q^{\dagger}, \beta^{\prime}\right\}=b^{\prime \dagger}} \\
\left.\left[Q^{\dagger}, \wp\right]=+i \beta^{\prime \dagger} d_{2} \quad\left[Q, \wp^{\dagger}\right]=-2 i \nabla_{0} \beta^{\prime}\right]=0 \\
\left\{Q, b^{\prime \dagger}\right] d_{2}^{\dagger} \beta^{\prime} \\
\left\{Q^{\dagger}, \xi\right\}=2 \nabla_{0} \wp+b^{\prime} d_{2}+i \beta^{\prime \dagger}\left(d_{2, a} \theta^{a}\right)
\end{gathered}
$$

So the $d^{2} \theta$ integral of $\beta^{\prime \dagger} \beta^{\prime}$ is

$$
b^{\prime} b^{\prime \dagger}-i\left(\nabla_{0} \beta^{\prime \dagger}\right) \beta^{\prime}+i \beta^{\prime \dagger}\left(\nabla_{0} \beta^{\prime}\right)
$$


and the $d^{2} \theta$ integral of $\wp \wp^{\dagger}$ contains five types of term:

- a 'target space magnetic field' term

$$
+i\left(\nabla_{0} \wp\right) \wp^{\dagger}-i \wp\left(\nabla_{0} \wp^{\dagger}\right)
$$

- quadratic terms for the gauge-invariant $\xi$ fermions:

$$
-\xi \xi^{\dagger}
$$

- mass terms for the $\beta^{\prime}$ fermions:

$$
+\beta^{\prime \dagger} d_{2} d_{2}^{\dagger} \beta^{\prime}
$$

- some F-terms giving rise to a bosonic potential for $\wp$ :

$$
i b^{\prime} d_{2} \wp^{\dagger}-i \wp d_{2}^{\dagger} b^{\prime \dagger}
$$

- and some cross terms between $\beta^{\prime}$ and $\wp$ which have no obvious role:

$$
-\beta^{\prime \dagger}\left(d_{2, a} \theta^{a}\right) \wp^{\dagger}-\wp\left(d_{2, a}^{\dagger} \theta^{a \dagger}\right) \beta^{\prime}
$$

- We also have the usual kinetic terms for the $\beta$-fermions:

$$
\int d^{2} \theta \beta^{\dagger} \beta=+b^{\dagger} b-i\left(\nabla_{0} \beta^{\dagger}\right) \beta+i \beta^{\dagger}\left(\nabla_{0} \beta\right)
$$

Also, the superpotential contributes the following component terms:

$$
\begin{aligned}
\int d \theta W & \equiv i \int d \theta \wp d_{1} \beta=i \int d \theta \wp d_{1} \beta \\
& =\xi d_{1} \beta+\beta^{\dagger} d_{1}^{\dagger} \xi^{\dagger} \\
& +\wp\left(d_{1, a} \theta^{a}\right) \beta+\beta^{\dagger}\left(d_{1, a}^{\dagger} \theta^{a \dagger}\right) \wp^{\dagger} \\
& +i \wp d_{1} b-i b^{\dagger} d_{1}^{\dagger} \wp^{\dagger} .
\end{aligned}
$$

The model with many nodes

For an arbitrary number of steps, the action is

$$
\int d^{2} \theta \sum_{n}\left(\beta_{(n)}^{\dagger} \beta_{(n)}+\wp_{(n+1)} \wp_{(n+1)}^{\dagger}\right)+\int d \theta W+\text { h.c. }
$$


and the on-shell supersymmetry transformations are

$$
\begin{aligned}
\left\{Q^{\dagger}, \beta_{(1)}\right\}=0 & \left\{Q, \beta_{(1)}\right\}=-i d_{1}^{\dagger} \cdot \wp_{(2)}^{\dagger} \\
\ldots & \\
\left\{Q^{\dagger}, \beta_{(n)}\right\}=i d_{n-1} \cdot \wp_{(n-1)}^{\dagger} & \left\{Q, \beta_{(n)}\right\}=-i d_{n}^{\dagger} \cdot \wp_{(n+1)}^{\dagger} \\
{\left[Q^{\dagger}, \wp_{(n+1)}^{\dagger}\right]=i d_{n} \cdot \beta_{(n)} } & {\left[Q, \wp_{(n+1)}^{\dagger}\right]=i d_{n+1}^{\dagger} \cdot \beta_{(n+2)} }
\end{aligned}
$$

Appendix B. Formulation with shift symmetries

In the main part of this chapter we have given a presentation of our models which clearly exhibits the spectrum of fields and their interactions. This presentation has the drawback that the supersymmetry transformations of the fields are complicated and the closure of the supersymmetry algebra is not manifest. In this appendix we present a formulation of our models in which the supersymmetry transformations of all fields are simple but which involves a number of nonlinearlyrealized gauge symmetries. The formulation of the models given in the main part of the chapter results from fixing these gauge symmetries. This appendix clarifies the relation between the modified chiral constraints and the "fermionic gauge symmetries" of $[235,247]$, and is how our models were initially constructed.

We again present the construction for a two-step sequence, (3.2). Introduce a fermi multiplet $\beta_{1}$ of bulk gauge charge $n_{1}$, a chiral multiplet $\tilde{\wp}_{2}$ of bulk gauge charge $-n_{2}$, and an unconstrained multiplet $V_{3}$ of gauge charge $n_{3}$ whose lowest component is a boson. The usual superpotential

$$
W=\tilde{\wp}_{2} d_{1} \beta
$$

respects the following shift symmetry, which we gauge:

$$
\begin{aligned}
& \tilde{\wp}_{1} \mapsto \tilde{\wp}_{1}+\Omega_{2} d_{2} \\
& V_{3} \mapsto V_{3}+\Omega_{2}
\end{aligned}
$$

The gauge parameter $\Omega_{2}$ is a chiral multiplet, $Q^{\dagger} \Omega_{2}=0$. Then the shift-symmetry invariant field $\wp_{2} \equiv \tilde{\wp}_{2}-V_{3} d_{2}$ satisifes the constraint $Q^{\dagger} \wp_{2}=\beta_{3}^{\dagger} d_{2}$ with $\beta_{3}^{\dagger} \equiv-Q^{\dagger} V_{3}$. $\beta_{3}^{\dagger}$ is gauge invariant and satisfies $Q^{\dagger} \beta_{3}^{\dagger}=0$. 
In order to extend the sequence by another step, enlarge the gauge symmetry.

Relax the condition $Q^{\dagger} \Omega_{2}=0$ on the gauge parameters and demand merely that $Q^{\dagger} \Omega_{2}=\Omega_{3} d_{3}$ for some $\Omega_{3}$ with $Q^{\dagger} \Omega_{3}=0$. Note again that the fact that the maps $d_{l}$ form a complex is crucial for closure of the superalgebra. Under this modified shift symmetry, the quantity $\wp_{2}$ is still gauge invariant, but $\tilde{\beta}_{3}^{\dagger} \equiv-Q^{\dagger} V_{3}$ is not.

Introduce a new gauge (i.e. unconstrained) multiplet $V_{4}$ (whose lowest component is a fermion) and assign it a transformation $\Sigma_{4} \mapsto \Sigma_{4}+\Omega_{3}$. Then $\beta_{3}^{\dagger} \equiv \tilde{\beta}_{3}^{\dagger}-\Sigma_{4} d_{3}$

is gauge invariant, and satisfies the deformed chiral constraint $Q^{\dagger} \beta_{3}^{\dagger}=\wp_{4} d_{3}$ where $\wp_{4} \equiv-Q^{\dagger} \Sigma_{4}$ is gauge invariant and annihilated by $Q^{\dagger}$. The generalization of this formulation to an arbitrary number of steps should be clear.

\section{Acknowledgements}

We thank Paul Aspinwall, Sarah Dean, Jacques Distler, Shamit Kachru, Sheldon Katz, Albion Lawrence, Dave Morrison and Eva Silverstein for discussions. The work of Simeon Hellerman is supported by the DOE under contract DE-AC0376SF00098 and by a DOE OJI grant. 


\section{Chapter 11: Matrix Theory on Projective Space}

Using linear sigma models for open strings, we derive supersymmetric actions for multiple pointlike D-branes probing the canonical bundles of toric surfaces. The moduli spaces of these worldvolume theories are symmetric products of the target space. In some instances, we are required to gauge nonlinearly-realized symmetries of the system. We comment on extensions of these techniques to the case of branes probing compact Calabi-Yau manifolds. We also discuss transitions in closed-string moduli space which reassign charges under the grading by worldsheet R-symmetry.

\section{Introduction}

M(atrix) theory and its AdS/CFT avatars have proven useful in defining the dynamics of quantum gravity on various spaces. Initially formulated in flat space [250], the theory has subsequently been developed to describe branes probing the geometry of orbifolds and their resolutions, as well as tori of diverse (but not too high) dimensions. Overall, the enterprise has proceeded in a rather ad hoc way, limited by our sparse understanding of the dynamics of branes transverse to nontrivial geometries.

Recently, a description of the dynamics of open strings in rather general spacetimes has been emerging [251]. In this approach, the wavefunctions for open string endpoints are sections of coherent sheaves, and a given D-brane configuration is an object in the derived category of coherent sheaves over the target space. There is a $\mathbb{Z}$ grading on the entries in this complex which encodes the relationship between spacetime supersymmetry and the worldsheet superconformal algebra. The problem of understanding the spectra of BPS objects is decomposed into one (F terms) which involves holomorphic data and can be solved using topological field theory, and (D terms) a harder stability problem which requires keeping track of the grading.

The unpublished material in this chapter was developed in collaboration with Simeon Hellerman. 
A useful tool for exploring the space of configurations (solutions at least to the F-term problem) is the open string linear sigma model $[232,178]$. In particular, the linear sigma models associated by the techniques of $[232,178]$ to two complexes $E$. and $F^{\cdot}$ which are quasiisomorphic can be shown to be IR equivalent. ${ }^{47}$ A current limitatation of the approach via linear models is the lack of control over the $U(1)$ R-symmetry in the infrared $\mathcal{N}=2$ superconformal algebra when there are extra global $U(1)$ symmetries present in the massive theory.

The goal of this chapter is to begin to combine the technologies of [251] and $[232,178]$ to study examples. In particular we find that we can construct quite explicitly the worldvolume theory for pointlike branes probing toric Calabi-Yau (CY) threefolds. We consider branes which are pointlike probes of the total space of the canonical line bundles over toric surfaces. This class of examples has a very simple construction via brane-antibrane physics. Relatedly, all worldvolume theories discussed in this chapter are invariant under a superalgebra containing four supercharges.

The fact that the target space has vanishing first Chern class enters the construction in only one way - it renders the theory supersymmetrizable. There is a close relationship between the fact that the target space is a CY threefold and the fact that we can impose the holomorphic constraints as the gradients of a single function. ${ }^{48}$ Namely, the assumption that the constraints on the massless fields in the worldvolume theory arise as gradients of some function implies that for each constraint $C$, the theory contains a field $L$ which lives in the space dual to that of the constraint (so that $\frac{\partial W}{\partial L}=C$ ). But we know that the light worldvolume fields themselves can be thought of as representatives of some first cohomology group (because you can think of them as deformations of a gauge connection, or of a tachyon map between adjacent nodes which carries R-charge one, or more generally because

47 The statement that $E^{\cdot}$ and $F^{\cdot}$ are quasiisomorphic says that there exists a map of complexes

$$
\rho: E^{\cdot} \rightarrow F^{\cdot}
$$

which induces an isomorphism on cohomology. Assembling the linear model for $E-F^{*}$. $\left(-F^{\cdot}\right.$ is the antibrane of $\left.F^{\cdot}\right)$, one finds that the map $\rho$ can be used to construct a gaugeinvariant worldsheet boundary superpotential. Turning on this operator gives mass to every boundary field - its condensation annihilates the two branes.

48 We thank Mike Douglas for explaining this to us. 
the string field has degree one) over the target space; further we know that the constraints lie in the corresponding second cohomology group (because the obstruction

to deforming a holomorphic connection is $F^{(0,2)}$, or because supersymmetry requires that the tachyon maps form a complex, or more generally because the equation of motion for the string field carries ghost number two). This means that for every element of

$$
H^{2}\left(X, V \otimes V^{\star}\right)
$$

we must able to find an element of the dual space, which by Serre duality is

$$
H^{1}\left(X, V \otimes V^{\star} \otimes \mathcal{K}_{X}\right)
$$

where $\mathcal{K}_{X}$ is the canonical bundle of the target space $X$. This can only be guaranteed if $X$ is $\mathcal{K}_{X}$ is trivial and $X$ is three-dimensional; namely, if $X$ is a Calabi-Yau threefold.

These theories also contain blown-up brane states which describe the nucleation of higher-dimensional brane-antibrane pairs which can be separated in directions transverse to the toric space.

\section{Matrix theory}

To our knowledge, no four-dimensional Minkowski vacuum of string theory has been given a nonperturbative definition. Such a definition of these backgrounds would be at least comforting, and possibly even useful. While the difficulties that arise in attempting a matrix definition of $\mathrm{M}$ theory on $T^{6}$ seem formidable $[252,253]$, recall that [254] suggest that the situation for matrix theory on a CY threefold whose holonomy is all of $S U(3)$ is better than that for $T^{6}$. In particular:

1. the light states in the (conjectured) DKPS limit for a $C Y_{3}$ do not have spin 2 ,

2. there is not an infinite (KK) tower of them, and

3. the worldvolume theory doesn't become $6+1$ dimensional in the limit, and therefore has a chance of decoupling from gravity. The theory is at most $3+1$ dimensional, and the three putative spatial dimensions are compact and string-scale (but maybe sometimes shrink). However momentum modes on the compact spatial volume of this theory are not BPS and one has no reason to believe their energies will shrink as the size of the original CY is scaled down; at energies below $\alpha^{\prime}$ we may integrate them out to obtain an effective quantum mechanics. 
In accord with the fact that we have little to say about issues of D-term stability, we do not make any claims about the kahler data in the worldvolume theory, which were the point at issue in $[255,256]$.

A problem that does not arise for matrix theories which enjoy more supersymmetry, but which will arise here, is the issue of what happens to the matrix description when the pointlike probe one is using can decay. The basic answer is that one must include in such a description also collections of branes and anti-branes ${ }^{49}$, which is the very purpose of the description via the derived category. We will see below that our descriptions in fact retain some of this additional information.

A cynical reading of our results is that we have found symplectic quotient descriptions of $N$-fold symmetric products of these CY threefolds.

The organization of the chapter is as follows. We warm up in $\S 2$ with branes on one dimensional projective space. This is a special case in that no superpotential term is required in the probe action. In $\S 3$, we study many-probe theories for $\mathcal{K}\left(\mathbb{P}^{2}\right)$, and for a cubic hypersurface thereof. $\S 4$ describes matrix models of the Hirzebruch surfaces, $\mathbb{F}_{n}$. $\S 5$ describes evidence that these nonrelativistic probe theories encode more of the physics than just their moduli space; namely, we interpret non-supersymmetric stationary points of the action in terms of nucleation of brane-antibrane pairs. MAYBE in $\S 6$ we mention some ideas about the emergent nature of the $\mathbb{Z}$ grading. In the conclusion we discuss the extension of these ideas to brane probes of compact Calabi-Yau manifolds.

We note that some of the quiver theories we write down have already appeared in the literature. In particular, the theories for $\mathbb{P}^{2}$ and for $\mathbb{P}^{1} \times \mathbb{P}^{1}$ were constructed in [258] in a similar context using a mirror description in terms of intersecting special Lagrangian three-cycles. These theories also appear in [259] and are implicit in [260]. Quiver theories for some of the other toric surfaces given in these references differ from the ones we construct. We comment in the concluding section on the group of dualities whose existence this suggests.

49 Such a viewpoint was recently advocated in [257]. 


\section{Branes on $\mathbb{C P}^{1}$ (not really)}

The simplest action to describe is that of zerobranes on the product of $\mathbb{C P}^{1}$ with a four-dimensional Minkowski factor. This theory is formally a dimensional reduction of a four-dimensional gauge theory. The relationship between the two theories is similar to that between the M(atrix) theory Lagrangian for zerobranes in 10-dimensional Minkowski space and the 10-dimensional super-Yang-Mills action in that the higher dimensional action is anomalous for general $S U(n)$, but its dimensional reductions are anomaly-free, and describe consistent brane actions.

The reader may be puzzled by the fact that $\mathbb{C P}^{1}$ is neither Ricci-flat nor of complex dimension three. We introduce this example mainly to give a flavor of the construction, but it can be embedded in string theory as a D-brane lagrangian in various ways. For instance if we consider type $I I A$ string theory on a Calabi-Yau which is a $K 3$-fibration of $\mathbb{C P}^{1}$, the lagrangian for $n$ fourbranes wrapped on the fiber has this form. However while the quiver is the simplest among the examples we consider, the embedding in string theory is more complicated than we wish to have to deal with, so we will just look at the Lagrangian and not worry too much about where it came from.

\subsection{Techniques}

We build a zerobrane from a brane and an antibrane wrapping the whole projective space:

$$
0 \rightarrow \mathcal{O} \stackrel{B(z)}{\longrightarrow} \mathcal{O}(1) \rightarrow 0
$$

where $B(z)$ is a linear polynomial in the homogenous coordinates $z^{1,2}$ of $\mathbb{P}^{1}$. There is one unit of gauge flux on the antibrane, so when the open string tachyon rolls to its minimum and the 2-branes annihilate one another, a D0-brane remains.

A useful framework for thinking about this system is the open string linear sigma model of [232]. Here one preserves two (B-type) worldsheet supercharges, and adds matter to the boundary of the string to construct the Chan-Paton bundle. Boundary condition changing vertex operators such as the tachyon mentioned above are bilinear in these boundary fields.

In this particular case, we consider the bulk linear model for $\mathbb{P}^{1}$ [186]

$$
\begin{array}{cccc} 
& z^{1} & z^{2} & p \\
\mathbb{C}^{*} & 1 & 1 & -2
\end{array}
$$


We have added the $p$ field to stabilize the kahler modulus of the projective space against renormalization. We will set $p_{\partial \Sigma}=0$ as a boundary condition in the UV. We will see that the worldsheet theory contains exactly marginal operators which correspond to shifts in this boundary condition.

\subsection{Worldvolume theory}

Massless worldvolume fields can be identified in the boundary linear sigma model by examining the cohomology of the worldsheet supercharge acting on operators of the massive theory [232]. This computes the Ext groups of the complex (2.1) with the maps turned off. Note that, just as in the closed string case where there can be "non-toric deformations," not all of the marginal operators are always obvious in the linear model.

The theory as we have defined it thus far results in a worldvolume gauge theory which contains two $U(1)$ factors, one combination of which is the decoupled center of mass $U(1)$. The complementary combination couples to a pair of chiral fields of the same charge, and will be the $U(1)$ implementing the quotient which turns $\mathbb{C}^{2}-\{(0,0)\}$ into $\mathbb{C P}^{1}$. The size of the projective space is determined by the magnitude of the Fayet-Iliopoulous coefficient for the relative $U(1)$ implementing the symplectic quotient. When this coupling is negative, this gauge theory is strongly coupled and a different description is more appropriate.

To model $N$ D0 branes probing this space, we simply append $N$ free ChanPaton labels to the ends of our strings. The gauge group of this theory is then $U(N)_{L} \times U(N)_{R}$ and the matter content is a pair of chiral superfields

$$
B_{i \tilde{j}}^{I}, \quad i, \tilde{j}=1, \ldots, N \quad I=1,2
$$

both transforming in the same representation $(N, \bar{N})$ of $U(N)_{L} \times U(N)_{R}$, and transforming as doublets under the $S U(2)$ global rotational invariance acting on the in$\operatorname{dex} I$. This global symmetry will implement the obvious $S U(2)$ rotations of the two-sphere, $\mathbb{P}^{1}$.

Note that unlike the $\mathcal{N}=1$ decomposition of an $\mathcal{N}=2$ hypermultiplet in the bifundamental, this pair of chiral multiplets transform the same, rather than dually to one another, under the pair of $S U(N)$ factors. That is, the quiver diagram is this: 


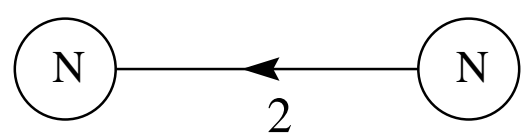

Fig. 19: Quiver diagram of the matter content of the $\mathbb{P}^{1}$ matrix theory.

Moduli space

The D-terms arising from the two gauge groups are

$$
\begin{aligned}
& D_{j}^{i}=-\sum_{I} B_{I i}^{\dagger k^{\prime}} B_{I j}^{k^{\prime}}-r \delta_{j}^{i} \\
& D_{j^{\prime}}^{i^{\prime}}=\sum_{I} B_{I k}^{i^{\prime}} B_{I k}^{\dagger j^{\prime}}-r^{\prime} \delta_{j^{\prime}}^{i^{\prime}}
\end{aligned}
$$

To parametrize the flat directions, start by writing

$$
B_{I}=\epsilon_{I J} Z^{J}
$$

This is motivated by the fact that the field $B$ represents the profile of the open string tachyon whose zero locus is the location of the zero brane:

$$
0=B_{I} Z^{I}
$$

is solved by (2.3). Next, let us work in a patch of the moduli space here $Z^{2}$ is invertible. In the abelian case, such a choice is always possible because at least one of the $Z \mathrm{~s}$ is always nonvanishing in the vacuum manifold (for positive FI parameters), or more generally from the description of the moduli space as a toric variety $\mathbb{C}^{N} \backslash F$ where $F$ is some subspace of $\mathbb{C}^{N}$ specified by the toric data. In the non-abelian case, the generalization of this condition is that the $Z$ matrices have no common kernel. If there are components of the vacuum manifold (when FI parameters are generic) where this condition fails, they have a very different geometry and we have not found them. Let

$$
Z^{2} W=Z^{1}
$$

define $W$, which will be our matrix local coordinate in this patch.

Next we show that $W$ commutes with its dagger using the D-terms in (2.2) above. Writing them in terms of $Z$ and suppressing indices, we get

$$
\begin{aligned}
0=D & =Z^{1} Z^{1 \dagger}+Z^{2} Z^{2 \dagger}-R^{2} \\
& =Z^{2} W\left(Z^{2} W\right)^{\dagger}+Z^{2} Z^{2 \dagger}-R^{2}
\end{aligned}
$$


Without loss of generatlity, we work in a patch where $Z^{2}$ is invertible. Multiplying both sides by $\left(Z^{2}\right)^{-1}$ on the left and by $\left(Z^{2 \dagger}\right)^{-1}$ on the right we get

$$
0=\left(Z^{2}\right)^{-1} D\left(Z^{2 \dagger}\right)^{-1}=W W^{\dagger}+1-R^{2}\left(Z^{2 \dagger} Z^{2}\right)^{-1}
$$

which gives

$$
Z^{2 \dagger} Z^{2}=R^{2}\left(W W^{\dagger}+1\right)^{-1}
$$

The other combination of D-terms then gives

$$
\begin{aligned}
D^{\prime} & =-Z^{1 \dagger} Z^{1}-Z^{2 \dagger} Z^{2}+R^{2} \\
& =-W^{\dagger} Z^{2 \dagger} Z^{2} W-Z^{2 \dagger} Z^{2}+R^{2} \\
& =-R^{2} W^{\dagger}\left(W W^{\dagger}+1\right)^{-1} W-R^{2}\left(W W^{\dagger}+1\right)^{-1}+R^{2} \\
& =-R^{2}\left[W, W^{\dagger}\right]+\mathcal{O}\left(W^{4}\right)
\end{aligned}
$$

where to get the third line we used (2.8) and in the last line we consider small fluctuations around the point $Z^{1}=0$, i.e. small $W$.

Thus, the worldsheet boundary linear model has led us to a field theory with four supercharges whose moduli space contains an $N$-fold symmetric product of $\mathbb{C I}^{1}$. We say "contains" because there are actually other massless fields on the worldvolume theory, related to motion in the $P$-field direction. We will come to terms with such degrees of freedom in the next section.

\section{Branes on $\mathbb{C P}^{2}$ (not really)}

Next we will try to study point-like branes on $\mathbb{C P}^{2}$. Clearly this is not a Ricci-flat space, so we must do something to stabilize its volume. We will therefore consisider strings propagating on the total space of the canonical bundle, $\mathcal{O}(-3)$, of $\mathbb{P}^{2}$, and we will restrict their endpoints to lie at the origin of the fiber direction.

The bulk linear model is defined by

$$
\begin{array}{ccccc} 
& z^{1} & z^{2} & z^{3} & p \\
\mathbb{C}^{*} & 1 & 1 & 1 & -3
\end{array}
$$

In addition to setting $\left.p\right|_{\partial \Sigma}=0$, we define our zerobrane by the complex

$$
0 \rightarrow \mathcal{O} \stackrel{\left[\begin{array}{l}
A_{1}(z) \\
A_{2}(z)
\end{array}\right]}{\longrightarrow} \mathcal{O}(1) \oplus \mathcal{O}(1) \stackrel{\left[B_{1}(z), B_{2}(z)\right]}{\longrightarrow} \mathcal{O}(2) \rightarrow 0
$$


This is indeed a complex if

$$
0=B_{1}(z) A_{1}(z)+B_{2}(z) A_{2}(z)
$$

In terms of the coefficients of $A_{i}(z)=A_{i I} z^{I}, B_{\bar{i}}(z)=B_{\bar{i}_{I}} z^{I}$, this is equivalent to

$$
0=\sum_{i}\left(B_{\bar{i} I} A_{i J}+(I \leftrightarrow J)\right)
$$

Calculating the $Q^{\dagger}$ cohomology, we find the field content defined by the quiver in Fig. 2.

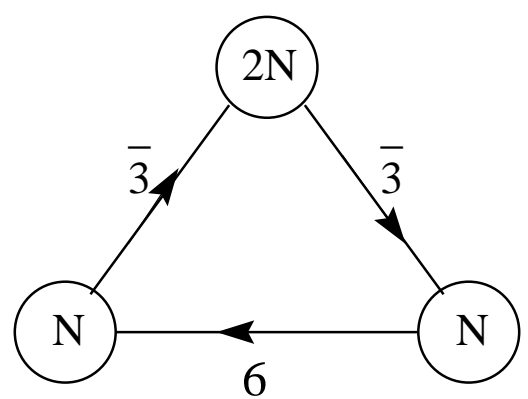

Fig. 20: The quiver diagram for matrix theory on $\mathbb{P}^{2}$.

The superpotential, as can be deduced from holomorphic Chern-Simons theory or because it is the only gauge-invariant object with the right properties, is (in matrix notation)

$$
W=\operatorname{Tr} S^{I J} B_{I} A_{J}
$$

\subsection{Moduli space}

We can parametrize the flat directions of the bosonic potential by $N \times N$ matrices $Z^{I}, P$ where

$$
\begin{aligned}
Z^{I} & =\epsilon^{I J K} B_{J} A_{K} \\
S^{I J} & =P Z^{I} Z^{J} .
\end{aligned}
$$

As for the example of $\mathbb{P}^{1}$ in $\S 2$, the definition of $Z$ is motivated by the fact that the zero locus of the open-string tachyon should be the location of the brane.

We begin by searching as before for 'pointlike' vacua, in which there exists a homogeneous coordinate on the base, say $Z^{3}$, which is invertible. 
Matrix local coords $W^{1,2}$ and $U$ are defined, in a patch where $Z^{3}$ is invertible, by

$$
W^{I}=\left(Z^{3}\right)^{-1} Z^{I}, \quad S^{I J}=U W^{I} W^{J}\left(Z^{3}\right)^{-1}
$$

That one of the three matrix projective coordinates be invertible like this we will call the assumption of a 'pointlike' vacuum. This name will justify itself a posteriori. We believe all D-term vacua in the large-base phase will turn out to be 'pointlike' in this sense.

Below we explain that in the case of multiple probes, all of the matrix local coordinates, including the fiber coordinate $U$, are forced to commute on the moduli space. But first, let us count charges, in the abelian case, to show that the extra dimension is actually the fiber of the canonical bundle. As we explained in the introduction, the fact that our probe worldvolume theory is $\mathcal{N}=1$ supersymmetric with a superpotential is related to the fact that the space being probed has trivial canonical bundle. Next we show that in fact the moduli space is the total space of said canonical bundle. There is a residual unbroken abelian gauge symmetry under which the $Z$ 's have unit charge. This implements a symplectic quotient on the moduli space, turning the space coordinatized by the $Z$ 's into a $\mathbb{C P}^{2}$. But what is the extra coordinate $P$ ? To determine its charge under this symplectic quotient, we note that the superpotential $W \sim S B A$ is neutral, $Z \sim B A$ has charge one, so $S$ has charge -1 . Since $S \sim P Z^{2}$, we conclude that $P$ has charge -3 . This means that it is precisely the coordinate on the fiber of the canonical bundle $\mathcal{O}(-3)$ over the $\mathbb{C P}^{2}$.

\subsection{Matricization}

Next we show that the ring of matrix functions is the same as the ring of regular functions on the $N$-fold symmetric product of $\mathcal{O}(-3) \rightarrow \mathbb{C P}^{2}$. First we show that $\left[w^{1}, w^{2}\right]=0$ in the $F$-term vacua.

Let $b \equiv w^{2} a, c \equiv w^{1} a$. If

$$
Z^{3} b=Z^{2} a, \quad Z^{3} c=Z^{1} a
$$

then what we need to show is that $Z^{1} b=Z^{2} a$. The key point is that since $Z^{3}$ is invertible and equal to $B_{1} A_{2}=-B_{2} A_{1}$, then $A_{1,2}$ have no kernel $n$-dimensional 
image, and $B_{1,2}$ have $n$-dimensional kernel. Combined with the fact that $B_{1} A_{1}=$ $B_{2} A_{2}=0$, this means that the kernel of $B_{1,2}$ equals the image of $A_{1,2}$.

Then we have

$$
B_{1} A_{2} b=-B_{2} A_{1} b=B_{3} A_{1} a=-B_{1} A_{3} a
$$

and likewise

$$
B_{1} A_{2} c=-B_{2} A_{1} c=B_{2} A_{3} a=-B_{3} A_{2} a
$$

so the vectors $A_{2} b+A_{3} a$ and $A_{1} c+A_{3} a$ lie in the kernels of $B_{1,2}$ and hence in the images of $A_{1,2}$, respectively. So $A_{3} a=A_{1} d-A_{2} b=A_{2} e-A_{1} c$. Multiplying on the left by $B_{1}$ and $B_{2}$ and gives $e=-b$ and $d=-c$. So we have $A_{1} c+A_{2} b+A_{3} a=0$, and multiplying on the left by $B_{3}$ gives $Z^{1} b=Z^{2} c$, as desired.

Next we show that both $Z^{I} S^{J K}$ and $S^{I J} Z^{K}$ are both totally symmetric in $I, J, K$.

The vanishing of $S^{I J} Z^{K}-(J \leftrightarrow K)$ is equivalent to the vanishing of $\epsilon_{J K L} S^{I J} Z^{K}$ which equals

$$
\epsilon_{K L J} \epsilon^{K M N} S^{I J} B_{M} A_{N}=S^{I J}\left(B_{L} A_{J}-(L \leftrightarrow J)\right)
$$

By the $F$-term condition for $S^{I L}$ this is equal to

$$
=-2 S^{I J} B_{J} A_{L}=-2\left(S^{I J} B_{J}\right) A_{L}
$$

and by the $F$-term condition for $A_{I}$ this vanishes. Likewise the quantity $\epsilon_{I J K} Z^{I} S^{J K}$ vanishes by the $F$-term conditions for $S$ and $B$.

This tells us two things. First of all, if we define $u \equiv S^{33} Z^{3}$ then we find that

$$
\begin{gathered}
u w^{i}=S^{33} Z^{i}=S^{3 i} Z^{3}=S^{i 3} Z^{3} \\
=\left(Z^{3}\right)^{-1} Z^{3} S^{i 3} Z^{3}=\left(Z^{3}\right)^{-1} Z^{i} S^{33} Z^{3}=\left(Z^{3}\right)^{-1} Z^{i} u=w^{i} u
\end{gathered}
$$

Second of all it tells us that

$$
S^{33} Z^{i}=S^{3 i} Z^{3}=S^{i 3} Z^{3}=u w^{i}
$$

and

$$
\begin{aligned}
S^{3 i} Z^{j} & =S^{j 3} Z^{i}=S^{i j} Z^{3}=S^{j 3} Z^{i}=(i \leftrightarrow j) \\
& =S^{3 i} Z^{3}\left(Z^{3}\right)^{-1} Z^{j}=u w^{i} w^{j}
\end{aligned}
$$

The result of this analysis is that the fiber direction $u$ is forced to commute with the other variables on the vacuum manifold. 


\subsection{Branes on a compact CY!}

Consider adding to the worldvolume superpotential the term

$$
\begin{aligned}
\Delta W & =G_{I J K} \operatorname{tr} \epsilon^{I J M} S^{J K} B_{L} A_{M} \\
& =G_{I J K} \operatorname{tr} P Z^{I} Z^{J} Z^{K}=\operatorname{tr} P G_{(3)}(Z)
\end{aligned}
$$

So the F-term equation from $P$ restricts the $Z$ 's to lie in the elliptic curve defined by the cubic equation $G_{(3)}(z)=0$.

Of course, we know how to make models of pointlike branes probing tori [261]. The only advance here is that this model contains a finite number of fields in the $\mathrm{UV}$, and, relatedly, its construction does not rely on translation-invariance.

\section{Branes on other toric surfaces}

\subsection{Branes on $\mathbb{P}^{1} \times \mathbb{P}^{1}$}

The bulk linear model for $\mathbb{P}^{1} \times \mathbb{P}^{1}$ has a gauge group with two abelian factors with the following charge vectors:

$\begin{array}{cccccc} & z^{1} & z^{2} & y^{1} & y^{2} & p \\ \mathbb{C}_{1}^{*} & 1 & 1 & 0 & 0 & -2 \\ \mathbb{C}_{2}^{*} & 0 & 0 & 1 & 1 & -2\end{array}$

Let $I=1,2$ and $\tilde{I}=1,2$ index the fundamentals of the $S U(2)$ global symmetries of the two $\mathbb{P}^{1}$ factors. A complex whose cohomology is a skyscraper sheaf at the point

$$
\left\{A(z)=A_{I} z^{I}=0\right\} \cap\left\{C(y)=C_{\tilde{I}} y^{\tilde{I}}=0\right\}
$$

is

$$
0 \rightarrow \mathcal{O}(0,0) \stackrel{\left[\begin{array}{l}
A(z) \\
C(y)
\end{array}\right]}{\longrightarrow} \mathcal{O}(1,0) \oplus \mathcal{O}(0,1) \stackrel{[B(y), D(z)]}{\longrightarrow} \mathcal{O}(1,1) \rightarrow 0 ;
$$

this is indeed a complex if

$$
B_{\tilde{J}} A_{I}+D_{J} C_{\tilde{I}}=0
$$

for all $I, \tilde{J}$.

Another vertex operator which exists is

$$
V_{S}=\beta^{\prime \dagger} \beta S_{I I^{\prime}} z^{I} y^{I^{\prime}} .
$$

It is difficult to distinguish this operator from its Serre dual:

$$
V_{S}=\beta^{\dagger} \beta S z^{I} y^{I^{\prime}} \eta^{\dagger I} \eta^{\dagger I^{\prime}} \eta^{\dagger P} .
$$




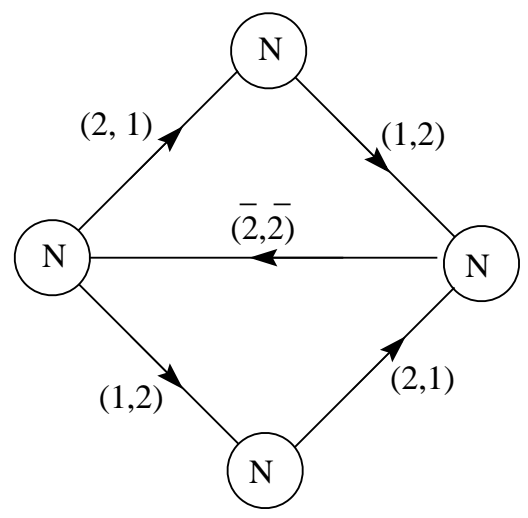

Fig. 21: The quiver diagram for matrix theory on $\mathbb{P}^{1} \times \mathbb{P}^{1}$. The fields are labeled by their representations of the global $S U(2) \times S U(2)$.

The superpotential is

$$
W=\operatorname{tr} S^{I \tilde{J}}\left(B_{\tilde{J}} A_{I}+D_{I} C_{\tilde{J}}\right)
$$

We can parametrize the flat directions by

$$
\begin{gathered}
A_{I}=\epsilon_{I J} Z^{J} \quad B_{\tilde{I}}=\epsilon_{\tilde{I} \tilde{J}} Y^{\tilde{J}} \\
C_{I}=-\epsilon_{I J} Z^{J} \quad D_{\tilde{I}}=\epsilon_{\tilde{I} \tilde{J}} Y^{\tilde{J}} \\
S^{I \tilde{J}}=P Z^{I} Y^{\tilde{J}} .
\end{gathered}
$$

Note that of the original quiver gauge group, two $\mathbb{C}^{\star}$ s act on these coordinates. One acts on the $Z$ s and the other acts on the $Y$ s. We have fixed others by choosing the ratios $A / C$, and $B / D$ to be -1 and +1 respectively. By counting charges under these unfixed $U(1)$ s we can see that the flat direction coordinatized by $P$ is indeed the fiber direction of the total space of the canonical bundle over $\mathbb{P}^{1} \times \mathbb{P}^{1}$. Since $W \sim S(B A+D C)$ is neutral under both, $B A \sim Z Y$ implies $S$ has charge $(-1,-1)$. Since $S \sim P Z Y, P$ has charge $(-2,-2)$ as befits the fiber of $\mathcal{O}(-2,-2) \rightarrow \mathbb{P}^{1} \times \mathbb{P}^{1}$.

\subsection{Branes on $\mathbb{F}_{n}$}

The bulk linear model for $\mathbb{F}_{n}\left(\mathbb{F}_{0}=\mathbb{P}^{1} \times \mathbb{P}^{1}\right)$ is defined by the toric data

$$
\begin{array}{cccccc} 
& z^{1} & z^{2} & y^{1} & y^{2} & p \\
\mathbb{C}_{1}^{*} & 1 & 1 & n & 0 & -2 \\
\mathbb{C}_{2}^{*} & 0 & 0 & 1 & 1 & -2
\end{array}
$$

Again, let $I=1,2$ the fundamental of the $S U(2)$ global symmetries of the base $\mathbb{P}^{1}$ coordinatized by $z$. 
We wish to place $N$ D-branes at the point

$$
\left\{A(z)=A_{I} z^{I}=0\right\} \cap\left\{Q_{(n)}(z) y^{2}-N y^{1}=0\right\}
$$

where $Q_{(n)}(z)=Q_{I_{1} \ldots I_{n}} z^{I_{1}} \cdots z^{I_{n}}$ is a degree $n$ polynomial in the homogeneous coordinates on the base. A complex whose cohomology is a skyscraper sheaf on the locus defined by (4.6) is

$$
0 \rightarrow \mathcal{O}(0,0) \stackrel{\left[\begin{array}{c}
A(z) \\
Q y^{1}-N y^{2}
\end{array}\right]}{\longrightarrow}(1,0) \oplus \mathcal{O}(n, 1) \stackrel{\left[R y^{1}-M y^{2}, B(y)\right]}{\longrightarrow} \mathcal{O}(n, 2) \rightarrow 0
$$

This is indeed a complex if

$$
\begin{array}{r}
R_{I_{1} \ldots I_{n}} A_{I_{n+1}}+B_{I_{1}} Q_{I_{2} \ldots I_{n+1}}+\text { perms } \\
=0 \\
A_{I} M+N B_{I}=0 .
\end{array}
$$

The superpotential is

$$
W=S^{I_{1} \ldots I_{n+1}}\left(R_{I_{1} \ldots I_{n}} A_{I_{n+1}}+B_{I_{1}} Q_{I_{2} \ldots I_{n+1}}\right)+T^{I}\left(A_{I} M+N B_{I}\right) .
$$

The flat directions of this superpotential are parametrized by $Z, P, Y$ where

$$
\begin{gathered}
A_{I}=\epsilon_{I J} Z^{J} \\
Q(Z) \equiv Q_{I_{1} \ldots I_{n}} Z^{I_{1}} \cdots Z^{I_{n}}=\left(Z_{I}^{\star} Z^{I}\right)^{n} Y^{1} \\
N=Y^{2}\left(Z_{I}^{\star} Z^{I}\right)^{n} \\
B_{I}=A_{I}, \quad R=-Q, \quad M=-N \\
S^{I_{1} \ldots I_{n+1}}=P Z^{I_{1}} \cdots Z^{I_{n+1}} Y^{2} \\
T^{I}=-P Z^{I} Y^{1}
\end{gathered}
$$

\subsection{Gauged shift symmetry}

Here we run into an interesting complication. The flat directions of the superpotential (4.9) fall into orbits of the symmetry $\Gamma$ which acts (nonlinearly) as

$$
\begin{aligned}
Q_{I_{1} \ldots I_{n}} & \mapsto Q_{I_{1} \ldots I_{n}}+A_{I_{1}} \Omega_{I_{2} \ldots I_{n}}+\text { perms } \\
R_{I_{1} \ldots I_{n}} & \mapsto R_{I_{1} \ldots I_{n}}+B_{I_{1}} \Omega_{I_{2} \ldots I_{n}}+\text { perms. }
\end{aligned}
$$

This symmetry arises from the fact that any function of $Z$ in the ideal generated by $A(z)$ will vanish at the point we are making by the complex (4.7). 
Therefore, in the correct worldvolume theory, whose moduli space really is $\mathcal{K}\left(\mathbb{F}_{n}\right)$, the shift symmetry $\Gamma$ is gauged. This is accomplished as follows.

To avoid proliferation of indices, we present the construction for the case of $\mathbb{F}^{1}$. In that case the action on $Q$ is

$$
Q_{I} \mapsto Q_{I}+\Omega_{I J} A_{J}
$$

We add some extra unconstrained fields $U_{I J}, T_{I J}$ and a new shift symmetry on which

$$
\begin{array}{r}
U_{I J} \mapsto U_{I J}+\Omega_{I J}+\omega_{I J}^{\dagger} \\
T_{I J} \mapsto T_{I J}+\omega_{I J} .
\end{array}
$$

Define

$$
\tilde{Q}_{I}=Q_{I}-U_{I J} A_{J}-T_{I J}^{\dagger} A_{J}
$$

to be the shift-symmetry invariant combination of these fields. We can then write kinetic terms in $4 \mathrm{~d} \mathcal{N}=1$ superspace by

$$
\int d^{4} \theta \tilde{Q}_{I}^{\dagger} \tilde{Q}_{I} \sim \int d^{4} \theta\left(Q^{\dagger} Q-U A Q^{\dagger}+\ldots\right) .
$$

Integrating out $U$ in this action gives a "nonlinearly realized D-term"

$$
V \sim\left|A_{I} Q_{I}^{\dagger}\right|^{2}
$$

which tells us that in the supersymmetric vacuum $0=A_{I}^{\dagger} Q_{I}$, but we have parametrized $A_{I}=\epsilon_{I J} Z^{J}$, so this says that

$$
0=\epsilon_{I J} Z^{J \star} Q_{I}
$$

which tells us that

$$
Q_{I} \propto Z^{I \star}
$$

\section{Reappearance of brane-antibrane constituents}

Consider again the model for $\mathbb{P}^{1}$. The potential arising from (2.2) has another stationary point other than the one where the D-terms vanish. This is the (unstable) point where the $B$ 's simply vanish. In this section we wish to give evidence for 
an interpretation of this state as one where the D2-brane and the anti-D2-brane wrapping the $\mathbb{P}^{1}$ from which we made the D0-brane have reappeared.

- In addition to its stable, supersymmetric vacuum, the theory has an unstable classical vacuum in which gauge symmetry is unbroken. The energy of this vacuum scales with the FI parameter, which is in turn the size of the $P^{1}$, in accord with the interpretation above.

- This vacuum has a Coulomb 'branch', where the constituents can be separated in a transverse direction. In the limit of infinite separation, we approach a supersymmetric state.

- We have discussed the $\mathbb{P}^{1}$ case for simplicity. These objects are also present in the toric surface examples. For example, in the model for $\mathbb{P}^{2}$, one can satisfy the F-term constraints by setting to zero the fields $B_{\bar{i} I}$, and the $i=$ 2 components of $A_{i I}$. The complex (3.1) then degenerates to the one-step complex

$$
0 \rightarrow \mathcal{O} \stackrel{\left[A_{1 I}\right]}{\longrightarrow} \mathcal{O}(1) \rightarrow 0 .
$$

- As mentioned in the introduction, the ability to see brane-antibrane nucleation is something that one wants from a less-supersymmetric matrix model since which are the stable probes will depend on the closed-string background.

\section{Regrading}

In [232] we pointed out that the simplest boundary linear model for a braneantibrane system (PLUS SOME ASSUMPTIONS ABOUT THE R-CHARGE IN THE UNBROKEN VACUUM) reproduces the worldvolume physics. Here, we refine this analysis by suggesting that MAYBE WE DON'T NEED TO RESPECT THE FUCKING ZZ-GRADING.

\section{Seiberg duality and its generalizations}

There are many ways to create a given lower dimensional brane configuration as the stable remnants of various unstable brane pairs. We point out that this fact fits nicely with what we know about

- the open string worldsheet and

- the geometry of sheaves and also with an expanded, less familiar version of Seiberg duality suggested by brane-antibrane physics. 


\subsection{Tachyon condensation and the open string worldsheet}

Unstable configurations with branes and antibranes can be described by linear $\sigma$-models which couple fields $\phi$ living in the bulk of the string worldsheet to bosonic and fermionic degrees of freedom $\wp, \beta$ which create and annihilate states of string endpoints on antibranes and branes, respectively.

Though there is no unbroken spacetime supersymmetry, the worldsheet dynamics still respect an exact superalgebra with a single complex supercharge $Q \neq Q^{\dagger}$. The relevant boundary operators corresponding to open string tachyons appear as boundary half-superspace integrals

$$
\int d \theta \wp f(\phi) \beta
$$

\section{Conclusions}

- OBVIOUSLY WE SHOULD DISCUSS OTHER PHASES OF THESE GAUGE THEORIES. and when they are weakly coupled descriptions and stuff.

- Perhaps the most attractive feature of this means of generating quiver theories is that it extends to the case of compact target space. We sketch the form this program should take and answer various objections along the way. E.G., winding strings ETC. ANSWER: IT'S NEAT, IT'S GOOD FOR SOMETHING OBVIOUSLY.

i.e. the Chern class fact about the coho of a sequence of sums of line bundles on a variety of degree greater than one.

- PERHAPS WRITE DOWN THE SHEAFY VARIABLES DESCRIPTION OF A SINGLE BRANE ON THE (FERMAT) QUINTIC? its only drawback is that it doesn't matricize easily.

$$
\int d \theta\left(\Gamma_{1} z_{1}+\Gamma_{2} z_{2}+\Gamma_{3} z_{3}+\Gamma_{4}\left(\frac{G_{5}(z)}{\left(z_{4}+\xi z_{5}\right)}\right)\right)
$$

where $\xi^{5}=1$.

\section{- DERIVATION FROM HOLOMORPHIC CS THEORY.}

\section{Acknowledgements}

We thank the Rutgers NHETC and Duke University for their hospitality. We thank Emanuel Diaconescu, Mike Douglas, Shamit Kachru and Eric Sharpe for discussions. 


\section{References}

[1] S. Kachru and J. McGreevy, "Supersymmetric three-cycles and (super)symmetry breaking," Phys. Rev. D 61, 026001 (2000) [arXiv:hep-th/9908135].

[2] K. Becker, M. Becker and A. Strominger, "Five-Branes, Membranes and Nonperturbative String Theory," Nucl. Phys. B456 (1995) 130, hep-th/9507158.

[3] J. Harvey and G. Moore, "Algebras, BPS States, and Strings," Nucl. Phys. B463 (1996) 315, hep-th/9510182;

J. Harvey and G. Moore, "On the Algebras of BPS States," Comm. Math. Phys. 197 (1998) 489, hep-th/9609017;

J. Harvey and G. Moore, "Superpotentials and Membrane Instantons," hepth/9907206.

[4] M. Bershadsky, V. Sadov and C. Vafa, "D-Strings on D-Manifolds," Nucl. Phys. B463 (1996) 398, hep-th/9510225;

M. Bershadsky, V. Sadov and C. Vafa, "D-Branes and Topological Field Theories," Nucl. Phys. B463 (1996) 420, hep-th/9511222.

[5] H. Ooguri, Y. Oz and Z. Yin, "D-Branes on Calabi-Yau Spaces and their Mirrors," Nucl. Phys. B477 (1996) 407, hep-th/9606112;

K. Becker, M. Becker, D. Morrison, H. Ooguri, Y. Oz and Z. Yin, "Supersymmetric Cycles in Exceptional Holonomy Manifolds and Calabi-Yau 4 Folds," Nucl. Phys. B480 (1996) 225, hep-th/9608116.

[6] A. Strominger, S.T. Yau and E. Zaslow, "Mirror Symmetry is T-Duality," Nucl. Phys. B479 (1996) 243, hep-th/9606040.

[7] N. Hitchin, "The moduli space of special Lagrangian submanifolds," math.dg/9711002;

N. Hitchin, "Lectures on Special Lagrangian Submanifolds," math.dg/9907034.

[8] C. Vafa, "Extending Mirror Conjecture to Calabi-Yau with Bundles," hepth/9804131.

[9] A. Karch, D. Lüst and A. Miemiec, "N=1 Supersymmetric Gauge Theories and Supersymmetric Three Cycles," hep-th/9810254.

[10] I. Brunner, M. Douglas, A. Lawrence and C. Romelsberger, "D-branes on the Quintic," hep-th/9906200.

[11] D. Joyce, "On counting special Lagrangian homology 3-spheres," hepth/9907013.

[12] R.C. McLean, Deformations and moduli of calibrated submanifolds. PhD thesis, Duke University, 1990.

[13] A. Sen, "BPS D-Branes on Non-Supersymmetric Cycles," hep-th/9812031.

[14] M. Berkooz, M. Douglas, and R. Leigh, "Branes Intersecting at Angles," Nucl. Phys. B480 (1996) 265, hep-th/9606139. 
[15] P. Fayet, "Higgs Model and Supersymmetry," Nuovo Cim. 31A (1976) 626.

[16] S. Kachru and E. Silverstein, "4d Conformal Field Theories and Strings on Orbifolds," Phys. Rev. Lett. 80 (1998) 4855, hep-th/9802183;

S. Kachru, J. Kumar and E. Silverstein, "Vacuum Energy Cancellation in a Nonsupersymmetric String," Phys. Rev. D59 (1999) 106004, hepth/9807076.

[17] For a review of this program with extensive references see: A. Sen, "Non-BPS States and Branes in String Theory," hep-th/9904207.

[18] S. Kachru, S. Katz, A. E. Lawrence and J. McGreevy, "Open string instantons and superpotentials," Phys. Rev. D 62, 026001 (2000) [arXiv:hepth/9912151].

[19] S. Shenker, "Another length scale in string theory?", hep-th/9509132.

[20] M.R. Douglas, D. Kabat, P.Pouliot and S. Shenker, "D-branes and short distances in string theory", Nucl. Phys. B485 (1997) 85, hep-th/9608024.

[21] M. Kontsevich, "Homological algebra of mirror symmetry", Proc. of the 1994 International Congress of Mathematicians, Birkhäuser (Boston) 1995; alggeom/9411018.

[22] D.R. Morrison, "Mirror symmetry and the type II string", Nucl. Phys. B Proc. Suppl. 46 (1996) 146, hep-th/9512016.

[23] A. Strominger, S.-T. Yau and E. Zaslow, "Mirror Symmetry is T-Duality", Nucl. Phys. B479 (1996) 243, hep-th/9606040.

[24] K. Becker, M. Becker and A. Strominger, "Five-branes, membranes and nonperturbative string theory," Nucl. Phys. B456 (1995) 130, hep-th/9507158.

[25] H. Ooguri, Y. Oz and Z. Yin, "D-branes on Calabi-Yau spaces and their mirrors", Nucl. Phys. B477 (1996) 407, hep-th/9606112.

[26] J.A. Harvey and G. Moore, "On the algebras of BPS states", Comm. Math. Phys. 197 (1998) 489, hep-th/9609017.

[27] E. Witten, "New Issues in Manifolds of $S U(3)$ Holonomy", Nucl. Phys. B268 (1986) 79.

[28] J. Distler and B. Greene, "Aspects of $(2,0)$ String Compactifications", Nucl. Phys. B304 (1988) 1.

[29] X.-G. Wen and E. Witten, "World-sheet instantons and the Peccei-Quinn symmetry", Phys. Lett. B166 (1986) 397.

[30] M. Dine, N. Seiberg, X.-G. Wen and E. Witten, "Non-perturbative effects on the string worldsheet I,II", Nucl. Phys. B278 (1986) 769; Nucl. Phys. B289 (1987) 319.

[31] E. Silverstein and E. Witten, "Criteria for Conformal Invariance of $(0,2)$ Models", Nucl. Phys. B444 (1995) 161, hep-th/9503212. 
[32] E. Witten, "Phases of N=2 Theories in Two-Dimensions", Nucl. Phys. B403 (1993) 159, hep-th/9301042.

[33] J. Distler and S. Kachru, "(0,2) Landau-Ginzburg Theory", Nucl. Phys. B413 (1994) 213, hep-th/9309110.

[34] N. Seiberg, "Electric-Magnetic Duality in Supersymmetric Non-Abelian Gauge Theories", Nucl. Phys. B435 (1995) 129, hep-th/9411149;

K. Intriligator and N. Seiberg, "Lectures on Supersymmetric Gauge Theories and Electric-Magnetic Duality", hep-th/9509066.

[35] E. Witten, "Nonperturbative Superpotentials in String Theory", Nucl. Phys. B474 (1996) 343, hep-th/9604030.

[36] P. Candelas, X. de la Ossa, P. Green and L. Parkes, "A Pair of Calabi-Yau Manifolds as an Exactly Soluble Superconformal Theory", Nucl. Phys. B359 (1991) 21.

[37] S. Kachru, N. Seiberg and E. Silverstein, "SUSY Gauge Dynamics and Singularities of 4d $\mathcal{N}=1$ String Vacua", Nucl. Phys. B480 (1996) 170, hepth/9605036;

S. Kachru and E. Silverstein, "Singularities, Gauge Dynamics and Nonperturbative Superpotentials in String Theory", Nucl. Phys. B482 (1996) 92, hep-th/9608194.

[38] I. Brunner, M.R. Douglas, A. Lawrence and C. Römelsberger, "D-branes on the Quintic", hep-th/9906200.

[39] S. Kachru, S. Katz, A. E. Lawrence and J. McGreevy, "Mirror symmetry for open strings," Phys. Rev. D 62, 126005 (2000) [arXiv:hep-th/0006047].

[40] E. Witten, "Chern-Simons gauge theory as a string theory", in The Floer Memorial Volume, H. Hofer et. al., eds., Birkhauser (1995), Boston, hepth/9207094.

[41] C. Vafa, "Extending mirror conjecture to Calabi-Yau with bundles", hepth/9804131.

[42] K. Fukaya, "Floer Homology and Mirror Symmetry I," 1999 Kyoto preprint, and references therein.

[43] R. McLean, "Deformations of Calibrated Submanifolds", Duke Univ. PhD thesis, Duke preprint 96-01: see www.math.duke.edu/preprints/1996.html.

[44] N.J. Hitchin, "The moduli space of special Lagrangian submanifolds," dg$\mathrm{ga} / 9711002$.

[45] D. Joyce, "On counting special Lagrangian homology 3-spheres", hepth/9907013.

[46] K. Kodaira, "A Theorem of Completeness of Characteristic Systems for Analytic Families of Compact Submanifolds of Compact Manifolds", Ann. Math. 
75 (1962) 146.

[47] D. Burns, "Some background and examples in deformation theory," in Complex Manifold Techniques in Theoretical Physics, D. Lerner and P. Sommers Eds., Pitman (1979).

[48] P. Candelas, X. de la Ossa, A. Font, S. Katz and D.R. Morrison, "Mirror symmetry for two-parameter models - I", Nucl. Phys. B416 (1994) 481, hep-th/9308083.

[49] S. Katz, "On the finiteness of rational curves on quintic threefolds", Comp. Math. 60, 151 (1986).

[50] S. Katz, "Rational Curves on Calabi-Yau Threefolds", in Mirror Symmetry I (S.-T. Yau, ed.), American Mathematical Society and International Press (1999); alg-geom/9312009.

[51] E. Witten, "Topological sigma models", Comm. Math. Phys. 118 (1988) 411.

[52] M. Reid, "Minimal Models of Canonical 3-folds", pp. 131-180, Advanced Studies in Pure Mathematics 1, ed. S. Iitaka, Kinokuniya (1983).

[53] R. Friedman, "Simultaneous resolution of threefold double points", Math. Ann. 274 (1986), 671-689.

[54] J. Bryan, S. Katz, and N.C. Leung, "Multiple covers and the integrality conjecture for rational curves in Calabi-Yau threefolds," math.AG/9911056.

[55] M. Bershadsky, V. Sadov and C. Vafa, "D-branes and Topological Field Theories," Nucl. Phys. B463 (1996) 420, hep-th/9511222.

[56] S. Katz, D.R. Morrison and M.R. Plesser, "Enhanced gauge symmetry in type II string theory", Nucl. Phys. B477 (1996) 105, hep-th/9601108.

[57] E. Witten, "Branes and the dynamics of QCD", Nucl. Phys. B507 (1997) 658-690, hep-th/9706109.

[58] J. Distler, "Resurrecting (0,2) compactifications", Phys. Lett. 188B (1987) 431.

[59] D. Friedan, E.J. Martinec and S.H. Shenker, "Conformal invariance, supersymmetry and string theory", Nucl. Phys. B271 (1986) 93.

[60] J.J. Atick, L.J. Dixon and A. Sen, "String calculation of Fayet-Iliopolous D-terms in arbitrary supersymmetric compactifications", Nucl. Phys. B292 (1987) 109

[61] H. Ooguri and C. Vafa, "Knot invariants and topological strings", hepth/9912123.

[62] Work in progress.

[63] R.P. Thomas, "An obstructed bundle on a Calabi-Yau 3-fold," math.AG/9903034.

[64] J.A. Harvey and G. Moore, "Superpotentials and membrane instantons," hep-th/9907026. 
[65] M.R. Douglas and M. Li, "D-brane realization of $\mathcal{N}=2$ super Yang-Mills theory in four dimensions," hep-th/9604041; A. Sen, "F-theory and orientifolds," Nucl. Phys. B475 (1996) 562, hep-th/9605150; T. Banks, M.R. Douglas and N. Seiberg, "Probing F-theory with branes," Phys. Lett. B387 (1996) 278, hep-th/9605199; N. Seiberg, "IR dynamics on branes and space-time geometry," Phys. Lett. B384 (1996) 81, hep-th/9606017.

[66] M. R. Douglas, "Topics in D Geometry," Class. Quant. Grav. 17 (2000) 1057, hep-th/9910170.

[67] I. Brunner, M.R. Douglas, A. Lawrence and C. Römelsberger, "D-branes on the Quintic," hep-th/9906200.

[68] P. Kaste, W. Lerche and C. Lutken, "D-branes on K3 Fibrations," hepth/9912147.

[69] S. Kachru, S. Katz, A. Lawrence and J. McGreevy, "Open String Instantons and Superpotentials," hep-th/9912151.

[70] E. Scheidegger, "D-branes on Some One Parameter and Two Parameter Calabi-Yau Hypersurfaces," JHEP 0004 (2000) 003, hep-th/9912188.

[71] M. Naka, M. Nozaki, "Boundary states in Gepner models," JHEP 0005 (2000) 027, hep-th/0001037.

[72] B. Greene and C. Lazaroiu, "Collapsing D-branes in Calabi-Yau Moduli Space I," hep-th/0001025.

[73] I. Brunner and V. Schomerus, "D-branes at Singular Curves of Calabi-Yau Compactifications," JHEP 0004 (2000) 020, hep-th/0001132.

[74] C. Lazaroiu, "Collapsing D-branes in One Parameter Models and Small/Large Radius Duality," hep-th/0002004.

[75] M. Douglas, B. Fiol and C. Romelsberger, "Stability and BPS Branes," hepth/0002037.

[76] S. Govindarajan and T. Jayaraman, "On the Landau-Ginzburg Description of Boundary CFTs and Special Lagrangian Submanifolds," hep-th/0003242.

[77] M. Douglas, B. Fiol and C. Romelsberger, "The Spectrum of BPS Branes on a Noncompact Calabi-Yau Space," hep-th/0003263.

[78] F. Denef, "Supergravity Flows and D-brane Stability," hep-th/0005049.

[79] K. Hori, A. Iqbal and C. Vafa, "D-branes and Mirror Symmetry," hepth/0005247.

[80] E. Witten, "Chern-Simons gauge theory as a string theory," in The Floer Memorial Volume, H. Hofer et. al., eds., Birkhauser (1995), Boston, hepth/9207094.

[81] C. Vafa, "Extending mirror conjecture to Calabi-Yau with bundles," hepth/9804131. 
[82] H. Ooguri and C. Vafa, "Knot invariants and topological strings," hepth/9912123.

[83] H. Ooguri, Y. Oz and Z. Yin, "D-branes on Calabi-Yau spaces and their mirrors," Nucl. Phys. B477 (1996) 407, hep-th/9606112.

[84] E. Witten, "Mirror manifolds and topological field theory," in Mirror Symmetry I, S.-T. Yau (ed.), American Mathematical Society (1998), hepth/9112056.

[85] J.A. Harvey and G. Moore, "On the algebras of BPS states," Comm. Math. Phys. 197 (1998) 489, hep-th/9609017.

[86] M. Marino, R. Minasian, G. Moore and A. Strominger, "Nonlinear instantons from supersymmetric p-branes," JHEP 0001 (2000) 005, hep-th/9911206.

[87] K. Kodaira, "A Theorem of Completeness of Characteristic Systems for Analytic Families of Compact Submanifolds of Compact Manifolds," Ann. Math. 75 (1962) 146.

[88] P. Candelas, X. de la Ossa, A. Font, S. Katz and D.R. Morrison, "Mirror symmetry for two-parameter models - I," Nucl. Phys. B416 (1994) 481, hep-th/9308083.

[89] S. Katz, D.R. Morrison and M.R. Plesser, "Enhanced gauge symmetry in type II string theory," Nucl. Phys. B477 (1996) 105, hep-th/9601108.

[90] R. McLean, "Deformations of Calibrated Submanifolds", Duke Univ. PhD thesis, Duke preprint 96-01: see www.math.duke.edu/preprints/1996.html.

[91] B. Greene and M. Plesser, "Duality in Calabi-Yau Moduli Space," Nucl. Phys. B338 (1990) 15.

[92] E. Witten, "Phases of $\mathrm{N}=2$ theories in two dimensions," Nucl. Phys. B403 (1993) 159, hep-th/9301042.

[93] S.-T. Yau, "Calabi's conjecture and some new results in algebraic geometry," Proc. Nat. Acad. Sci. U.S.A. 74 (1977) 1798.

[94] A. Strominger, S.-T. Yau and E. Zaslow, "Mirror Symmetry is T-Duality," Nucl. Phys. B479 (1996) 243, hep-th/9606040.

[95] P.S. Aspinwall, B.R. Greene and D.R. Morrison, "The monomial-divisor mirror map," Internat. Math. Res. Notices 93 (1993) 319, alg-geom/9309007.

[96] M. Kontsevich, "Homological algebra of mirror symmetry," Proc. of the 1994 International Congress of Mathematicians, Birkhäuser (Boston) 1995, alggeom/9411018.

[97] T. Banks, L.J. Dixon, D. Friedan and E. Martinec, "Phenomenology and conformal field theory: or, can string theory predict the weak mixing angle?," Nucl. Phys. B299 (1988) 613.

[98] J. McGreevy, L. Susskind and N. Toumbas, "Invasion of the giant gravitons 
from anti-de Sitter space," JHEP 0006, 008 (2000) [arXiv:hep-th/0003075].

[99] L. Susskind and E. Witten, "The holographic bound in anti-de Sitter space," arXiv:hep-th/9805114.

[100] L. Susskind, "The World as a hologram," J. Math. Phys. 36, 6377 (1995) [arXiv:hep-th/9409089].

[101] L. Susskind, arXiv:hep-th/9511116.

[102] T. Banks, W. Fischler, S. H. Shenker and L. Susskind, "M theory as a matrix model: A conjecture," Phys. Rev. D 55, 5112 (1997) [arXiv:hep-th/9610043].

[103] Leonard Susskind, "Strings, Black Holes and Lorentz Contraction," hepth/9308139 Journal-ref: Phys.Rev. D49 (1994) 6606-6611

[104] Antal Jevicki and Tamiaki Yoneya, "Space-Time Uncertainty Principle and Conformal Symmetry in D-Particle Dynamics," hep-th/9805069 Journal-ref: Nucl.Phys. B535 (1998) 335-348

[105] Miao Li, Tamiaki Yoneya, "D-Particle Dynamics and The Space-Time Uncertainty Relation," hep-th/9611072 Journal-ref: Phys.Rev.Lett. 78 (1997) 1219-1222

[106] Sheikh-Jabbari, "Open Strings in a B-field Background as Electric Dipoles," hep-th/9901080 Journal-ref: Phys.Lett. B455 (1999) 129-134

[107] Daniela Bigatti, Leonard Susskind, "Magnetic fields, branes and noncommutative geometry," hep-th/9908056

[108] Shiraz Minwalla, Mark Van Raamsdonk, Nathan Seiberg, "Noncommutative Perturbative Dynamics," hep-th/9912072

[109] Mark Van Raamsdonk, Nathan Seiberg, "Comments on Noncommutative Perturbative Dynamics," hep-th/0002186

[110] Alec Matusis, Leonard Susskind, Nicolaos Toumbas, "The IR/UV Connection in the Non-Commutative Gauge Theories," hep-th/0002075

[111] Juan Maldacena, Andrew Strominger, " $\mathrm{AdS}_{3}$ Black Holes and a Stringy Exclusion Principle," hep-th/9804085 Journal-ref: JHEP 9812 (1998) 005

[112] Pei-Ming Ho, Sanjaye Ramgoolam, Radu Tatar, "Quantum Spacetimes and Finite N Effects in 4D Super Yang-Mills Theories," hep-th/9907145

[113] Antal Jevicki, Sanjaye Ramgoolam, "Non commutative gravity from the ADS/CFT correspondence," hep-th/9902059 Journal-ref: JHEP 9904 (1999) 032

[114] See, for example, J. Madore, An Introduction to Noncommutative Differential Geometry and its Physical Applications, Cambridge U. Press, 2nd edition (1999).

[115] Juan Maldacena, "The Large N Limit of Superconformal Field Theories and Supergravity," hep-th/9711200 
[116] R.C. Myers, "Dielectric-Branes," hep-th/9910053 Journal-ref: JHEP 9912 (1999) 022

[117] Joseph Polchinski, Matthew J. Strassler, "The String Dual of a Confining Four-Dimensional Gauge Theory," hep-th/0003136.

[118] Juan Maldacena, Leonard Susskind, "D-Branes and Fat Black Holes," hepth/9604042 Journal-ref: Nucl.Phys. B475 (1996) 679-690; Juan Maldacena, "Statistical Entropy of Near Extremal Five-Branes," hep-th/9605016 Journal-ref: Nucl.Phys. B477 (1996) 168-174

[119] Emil Martinec, "Conformal Field Theory, Geometry, and Entropy," hepth/9809021.

[120] S. Kachru and J. McGreevy, "M-theory on manifolds of G(2) holonomy and type IIA orientifolds," JHEP 0106, 027 (2001) [arXiv:hep-th/0103223].

[121] D.D. Joyce, "Compact Riemannian 7-manifolds with $G_{2}$ Holonomy, I," J. Diff. Geom. 43 (1996) 291.

[122] D.D. Joyce, "Compact Riemannian 7-manifolds with $G_{2}$ Holonomy, II," J. Diff. Geom. 43 (1996) 329.

[123] J. Harvey and G. Moore, "Superpotentials and Membrane Instantons," hepth/9907026.

[124] A. Sen, "F-theory and Orientifolds," Nucl. Phys. B475 (1996) 562; A. Sen, "Orientifold Limit of F-theory Vacua," Phys. Rev. D55 (1997) 7345, hepth/9702165.

[125] R. Gopakumar and S. Mukhi, "Orbifold and Orientifold Compactifications of F-theory and M-theory to Six and Four Dimensions," Nucl. Phys. B479 (1996) 260, hep-th/9607057.

[126] M. Atiyah, J. Maldacena and C. Vafa, "An M-theory Flop as a Large N Duality," hep-th/0011256.

[127] S. Sinha and C. Vafa, "SO and Sp Chern-Simons at Large N," hepth/0012136.

[128] B.S. Acharya, "On Realizing $\mathcal{N}=1$ Super Yang-Mills in M-theory," hepth/0011089; "Confining Strings from G(2) Holonomy Space-Times," hepth/0101206;

B.S. Acharya and C. Vafa, "On Domain Walls of $\mathcal{N}=1$ Supersymmetric Yang-Mills in Four-Dimensions," hep-th/0103011.

[129] F. Cachazo, K. Intriligator and C. Vafa, "A Large N Duality via a Geometric Transition," hep-th/0103067.

[130] E. Witten, "M-theory Dynamics on Manifolds of $G_{2}$ Holonomy," talk presented March 3, 2001 at "Heterotic Dreams and Asymptotic Visions," the 60th birthday celebration for David Gross, ITP, Santa Barbara. 
[131] J. Edelstein and C. Nunez, "D6 branes and M theory geometrical transitions from gauged supergravity," hep-th/0103167.

[132] J. Gomis, "D-branes, Holonomy and M-theory," hep-th/0103115.

[133] B.S. Acharya, "M-theory, Joyce Orbifolds and Super Yang-Mills," Adv. Th. Math. Phys. 3 (1999) 227, hep-th/9812205.

[134] H. Partouche and B. Pioline, "Rolling Among $G_{2}$ Vacua," JHEP 0103 (2001) 005, hep-th/0011130.

[135] A. Sen, "A Note on Enhanced Gauge Symmetries in M and String Theory," JHEP 9709 (1997) 001, hep-th/9707123.

[136] C. Vafa and E. Witten, "Dual String Pairs with $\mathcal{N}=1$ and $\mathcal{N}=2 \mathrm{Su}-$ persymmetry in Four Dimensions," Nucl. Phys. Proc. Suppl. 46 (1996) 225, hep-th/9507050.

[137] A. Klemm, W. Lerche and P. Mayr, "K3 Fibrations and Heterotic-Type II String Duality," Phys. Lett. B357 (1995) 313, hep-th/9506112.

[138] S. Kachru and C. Vafa, "Exact Results for $\mathcal{N}=2$ Compactifications of Heterotic Strings," Nucl. Phys. B450 (1995) 69, hep-th/9505105.

[139] P. Aspinwall and M. Gross, "Heterotic-Heterotic String Duality and Multiple K3 Fibrations," Phys. Lett. B382 (1996) 81, hep-th/9602118.

[140] D. Morrison and C. Vafa, "Compactifications of F-theory on Calabi-Yau Threefolds, I," Nucl. Phys. B473 (1996) 74, hep-th/9602114.

[141] M. Douglas, "Branes within branes," hep-th/9512077.

[142] H. Ooguri and C. Vafa, "Knot Invariants and Topological Strings," Nucl. Phys. B577 (2000) 419, hep-th/9912123.

[143] M. Aganagic and C. Vafa, "Mirror Symmetry, D-branes and Counting Holomorphic Discs," hep-th/0012041.

[144] S. Kachru and E. Silverstein, "Chirality Changing Phase Transitions in 4d String Vacua," Nucl. Phys. B504 (1997) 272, hep-th/9704185;

B. Ovrut, T. Pantev and J. Park, "Small Instanton Transitions in Heterotic M-theory," hep-th/0001133.

[145] A. Strominger, S.T. Yau and E. Zaslow, "Mirror Symmetry Is T-Duality," Nucl. Phys. B479(1996) 243, hep-th/9606040.

[146] S. Hellerman and J. McGreevy, "D(NA)-branes," JHEP 0110, 021 (2001) [arXiv:hep-th/0104010].

[147] A. Bernevig, J. Brodie, L. Susskind and N. Toumbas, "How Bob Laughlin Tamed the Giant Graviton from Taub-NUT Space," hep-th/0010105.

[148] L. Susskind, "The Quantum Hall Fluid and Non-Commutative Chern Simons Theory," hep-th/0101029. 
[149] A. Polychronakos, "Quantum Hall states as matrix Chern-Simons theory," hep-th/0103013.

[150] S. Hellerman and M. van Raamsdonk, "Quantum Hall Physics = Noncommutative Field Theory," hep-th/0103179.

[151] A. Hanany and E. Witten, "Type IIB superstrings, BPS monopoles and three-dimensional gauge dynamics," hep-th/9611230.

[152] M. Gaberdiel and B. Zwiebach, "Exceptional groups from open strings," hep-th/9709013.

[153] R. Gregory and R. Laflamme, "Black strings and p-branes are unstable," hep-th/9301052.

[154] G. Gibbons, M. Green, M. Perry, "Instantons and seven-branes in Type IIB superstring theory," hep-th/9511080.

[155] C. Johnson, unpublished.

[156] M. Dine and N. Seiberg, "Is The Superstring Weakly Coupled?," Phys. Lett. B 162, 299 (1985).

[157] M. Dine, Y. Nir and Y. Shadmi, "Enhanced symmetries and the ground state of string theory," Phys. Lett. B 438, 61 (1998) [arXiv:hep-th/9806124].

[158] B. R. Greene, A. D. Shapere, C. Vafa and S. T. Yau, "Stringy Cosmic Strings And Noncompact Calabi-Yau Manifolds," Nucl. Phys. B 337, 1 (1990).

[159] C. G. Callan, J. A. Harvey and A. Strominger, hep-th/9112030.

[160] A. Strominger, S. T. Yau and E. Zaslow, Nucl. Phys. B 479, 243 (1996) [arXiv:hep-th/9606040].

[161] K. Kodaira, Annals of Math. 77 (1963) 563; Annals of Math. 78 (1963) 1.

[162] H. Ooguri and C. Vafa, "Two-Dimensional Black Hole and Singularities of CY Manifolds," Nucl. Phys. B 463, 55 (1996) [arXiv:hep-th/9511164].

[163] J. D. Blum and K. A. Intriligator, "Consistency conditions for branes at orbifold singularities," Nucl. Phys. B 506, 223 (1997) [arXiv:hep-th/9705030].

[164] J. D. Blum and K. A. Intriligator, "New phases of string theory and 6d RG fixed points via branes at orbifold singularities," Nucl. Phys. B 506, 199 (1997) [arXiv:hep-th/9705044].

[165] K. A. Intriligator, "New string theories in six dimensions via branes at orbifold singularities," Adv. Theor. Math. Phys. 1, 271 (1998) [arXiv:hepth/9708117].

[166] M. R. Douglas and G. W. Moore, "D-branes, Quivers, and ALE Instantons," arXiv:hep-th/9603167.

[167] C. Vafa, "Evidence for F-Theory," Nucl. Phys. B 469, 403 (1996) [arXiv:hepth/9602022].

[168] M. F. Atiyah and R. Bott, "A Lefschetz Fixed Point Formula for Elliptic 
Differential Operators," Bull. Am. Math. Soc. 72 (1968) 531; M. F. Atiyah and G. B. Segal, "The Index Of Elliptic Operators. 2," Annals Math. 87, 531 (1968).

[169] R. Friedman, J. Morgan and E. Witten, "Vector bundles and F theory," Commun. Math. Phys. 187, 679 (1997) [arXiv:hep-th/9701162].

[170] T. Eguchi, P. B. Gilkey and A. J. Hanson, "Gravitation, Gauge Theories And Differential Geometry," Phys. Rept. 66, 213 (1980).

[171] O. J. Ganor and A. Hanany, "Small $E_{8}$ Instantons and Tensionless Noncritical Strings," Nucl. Phys. B 474, 122 (1996) [arXiv:hep-th/9602120].

[172] N. Seiberg and E. Witten, "Comments on String Dynamics in Six Dimensions," Nucl. Phys. B 471, 121 (1996) [arXiv:hep-th/9603003].

[173] S. Kachru and E. Silverstein, "Chirality-changing phase transitions in $4 \mathrm{~d}$ string vacua," Nucl. Phys. B 504, 272 (1997) [arXiv:hep-th/9704185].

[174] A. Sen, "M-Theory on $\left(K 3 \times S^{1}\right) / Z_{2}$," Phys. Rev. D 53, 6725 (1996) [arXiv:hep-th/9602010].

[175] A. Dabholkar and J. Park, "An Orientifold of Type-IIB Theory on K3," Nucl. Phys. B 472, 207 (1996) [arXiv:hep-th/9602030].

[176] D. R. Morrison and C. Vafa, "Compactifications of F-Theory on Calabi-Yau Threefolds - II," Nucl. Phys. B 476, 437 (1996) [arXiv:hep-th/9603161].

[177] F. Giani and M. Pernici, Phys. Rev. D 30, 325 (1984).

[178] S. Hellerman and J. McGreevy, "Linear sigma model toolshed for D-brane physics," JHEP 0110, 002 (2001) [arXiv:hep-th/0104100].

[179] B.R. Greene, "D-brane topology changing transitions," Nucl. Phys. B525 (1998) 284, hep-th/9711124.

[180] T. Muto, "D-branes at orbifolds and topology change," Nucl. Phys. 521 (1998) 183, hep-th/9711090.

[181] S. Kachru, A. Lawrence and E. Silverstein, "On the matrix description of Calabi-Yau compactifications," Phys. Rev. Lett. 80 (1998) 2996, hepth/9712223.

[182] I. Brunner, M.R. Douglas, A. Lawrence and C. Römelsberger, "D-branes on the Quintic," JHEP 0008 (2000) 015, hep-th/9906200.

[183] M.R. Douglas, B. Fiol and C. Römelsberger, "Stability and BPS branes," hep-th/0002037.

[184] F. Denef, "Supergravity Flows and D-brane Stability," JHEP 0008 (2000) 050, hep-th/0005049;

F. Denef, B. Greene and M. Raugas, "Split Attractor Flows and the Spectrum of BPS D-branes on the Quintic," JHEP 0105 (2001) 012, hep-th/0101135;

F. Denef, "(Dis)assembling Special Lagrangians," hep-th/0107152. 
[185] M.R. Douglas, B. Fiol and C. Römelsberger, "The spectrum of BPS branes on a noncompact Calabi-Yau," hep-th/0003263.

[186] E. Witten, "Phases of N=2 theories in two dimensions," Nucl. Phys. B403 (1993) 159, hep-th/9301042.

[187] P. Aspinwall, B. Greene and D. Morrison, "Calabi-Yau moduli space, mirror manifolds and space-time topology change in string theory," Nucl. Phys. B416 (1994) 414, hep-th/9309097.

[188] T.-M. Chiang and B.R. Greene, "Phases of mirror symmetry," Strings '95 proceedings Future Perspectives in String Theory (World Scientific, New Jersey) 1996; hep-th/9509049.

[189] B. Greene, C. Vafa and N. Warner, "Calabi-Yau manifolds and renormalization group flows," Nucl. Phys. B324 (1989) 371;

E. Martinec, "Criticality, Catastrophes and Compactifications," in Brink, L. (ed.) et al., Physics and Mathematics of Strings 383.

[190] E. Silverstein and E. Witten, "Criteria for conformal invariance of $(0,2)$ models," Nucl. Phys. 444 (1995) 161, hep-th/9503212.

[191] K. Hori, A. Iqbal and C. Vafa, "D-Branes and mirror symmetry," hepth/0005247.

[192] S. Govindarajan, T. Jayaraman, and T. Sarkar, "On D-branes from gauged linear sigma models," hep-th/0007075.

[193] K. Becker, M. Becker and A. Strominger, "Five-branes, membranes and nonperturbative string theory," Nucl. Phys. B456 (1995) 130, hep-th/9507158.

[194] H. Ooguri, Y. Oz and Z. Yin, "D-branes on Calabi-Yau spaces and their mirrors," Nucl. Phys. B477 (1996) 407, hep-th/9606112.

[195] D.-E. Diaconescu and M.R. Douglas, "D-branes on stringy Calabi-Yau manifolds," hep-th/0006224.

[196] J. Distler and S. Kachru, "(0,2) Landau-Ginzburg Theory," Nucl. Phys. B413 (1993) 213, hep-th/9309110.

[197] Talk given by S. Kachru at Strings 2000, http://feynman.physics . lsa. umich.edu/cgibin/s2ktalk.cgi?kachru

[198] P. Mayr, "Phases of supersymmetric D-branes on Kaehler manifolds and the McKay correspondence," hep-th/0010223.

[199] K. Hori, "Linear models of supersymmetric D-branes," hep-th/0012179.

[200] S. Govindarajan, T. Jayaraman, "D-branes, exceptional sheaves and quivers on Calabi-Yau manifolds," Nucl. Phys. B600 (2001) 457, hep-th/0010196; "Boundary fermions, coherent sheaves and D-branes on Calabi-Yau manifolds," hep-th/0104126. 
[201] J. Distler, "Notes on $(0,2)$ superconformal field theories," Trieste HEP Cosmology 1994:0322-351, hep-th/9502012.

[202] P. Fendley, H. Saleur and N.P. Warner, "Exact solution of a massless scalar field with a relevant boundary interaction," Nucl. Phys. B430 (1994) 577, hep-th/9406125;

J.A. Harvey, D. Kutasov and E.J. Martinec, "On the relevance of tachyons," hep-th/0003101.

[203] D. Gepner, "Exactly Solvable String Compactifications on Manifolds of SU $(N)$ Holonomy," Phys. Lett. B199 (1987) 380.

[204] N. Seiberg, Phys. Lett. B318, 469 (1993), hep-ph/9309335.

[205] E. Witten, "On the Landau-Ginzburg description of $\mathrm{N}=2$ minimal models," Int. J. Mod. Phys. A9 (1994) 4783, hep-th/9304026.

[206] S. Kachru and E. Witten, "Computing the Complete Massless Spectrum of a Landau-Ginzburg Orbifold," hep-th/9307038.

[207] E. Witten, "Mirror manifolds and topological field theory," in Mirror Symmetry I, S.-T. Yau (ed.), American Mathematical Society (1998), hepth/9112056.

[208] W. Lerche, C. Vafa and N.P. Warner, "Chiral rings in $\mathcal{N}=2$ superconformal theories," Nucl. Phys. B324 (1989) 427.

[209] C. Lazariou, "String field theory and brane superpotentials," hep-th/0107162;

A. Tomasiello, "A-infinity structure and superpotentials," hep-th/0107195.

[210] D. Morrison and M.R. Plesser, "Summing the Instantons: Quantum Cohomology and Mirror Symmetry in Toric Varieties," Nucl. Phys. B440 (1995) 279, hep-th/9412236.

[211] S. Hellerman and J. McGreevy, work in progress.

[212] N. Warner, "Supersymmetry in boundary integrable models," Nucl. Phys. B450 (1995) 663, hep-th/9506064

[213] R. I. Nepomechie, "The boundary $\mathrm{N}=2$ supersymmetric sine-Gordon model," Phys. Lett. B 516, 376 (2001); hep-th/0106207, "Boundary S matrices with $\mathrm{N}=2$ supersymmetry," Phys. Lett. B 516, 161 (2001), hepth/0106223.

[214] J. Polchinski, String Theory, two vols., Cambridge (1999).

[215] M. Douglas, "D-branes, Categories and $\mathcal{N}=1$ Supersymmetry," hepth/0011017.

[216] P. Aspinwall and A. Lawrence, "Derived Categories and Zero-Brane Stability," hep-th/0104147.

[217] C. I. Lazaroiu, JHEP 0106, 052 (2001), hep-th/0102122; "Unitarity, D- 
brane dynamics and D-brane categories," hep-th/0102183; D.-E. Diaconescu, "Enhanced D-brane Categories from String Field Theory," hep-th/0104200; C. I. Lazaroiu, JHEP 0106, 064 (2001), hep-th/0105063; C. I. Lazaroiu, R. Roiban and D. Vaman, hep-th/0107063.

[218] P. Griffiths and J. Harris, Principles of Algebraic Geometry, John Wiley and Sons (1978) NY.

[219] D. Kutasov, G. Moore and M. Mariño, "Some exact results on tachyon condensation in string field theory," JHEP 0010 (2000) 045, hep-th/0009148.

[220] T. Gomez and E. Sharpe, "D-branes and Scheme Theory," hep-th/0008150.

[221] P. Seidel and R.P. Thomas, "Braid group actions on derived categories of coherent sheaves," math.AG/0001043; P.S. Aspinwall, "Some navigation rules for D-brane monodromy," hep-th/0102198; P. Horja, "Derived category automorphisms from mirror symmetry," math.AG/0103231.

[222] P. Candelas, X.C. de la Ossa, P.S. Green and L. Parkes, "A pair of Calabi-Yau manifolds as an exactly solvable superconformal field theory," Nucl. Phys. 359 (1991) 21.

[223] E. Witten and J. Bagger, "Quantization of Newton's Constant in Certain Supergravity Theories," Phys. Lett. B115 (1982) 202.

[224] E. Witten, "Small Instantons in String Theory," Nucl. Phys. B460 (1996) 541, hep-th/9511030.

[225] P. Fayet, "Higgs Model and Supersymmetry," Nuovo Cim. A31 (1976) 626.

[226] D. Joyce, "On counting special lagrangian homology three spheres," hepth/9907013.

[227] E. Sharpe, "Kähler cone substructure," Adv.Theor.Math.Phys. 2 (1999) 1441, hep-th/9810064.

[228] S. Mukai, "Symplectic structure of the moduli space of sheaves on an abelian or K3 surface," Invent. Math. 77 (1984) 101.

[229] M. Aganagic and C. Vafa, "Mirror Symmetry, D-branes and Counting Holomorphic Discs," hep-th/0012041;

M. Aganagic, A. Klemm and C. Vafa, "Disk Instantons, Mirror Symmetry and the Duality Web," hep-th/0105045.

[230] S. Govindarajan, T. Jayaraman and T. Sarkar, "Disc Instantons in Linear Sigma Models," hep-th/0108234; P. Mayr, "N = 1 mirror symmetry and open/closed string duality," hep-th/0108229.

[231] D. Eisenbud, Commutative Algebra with a View Toward Algebraic Geometry, Springer (1996).

[232] S. Hellerman, S. Kachru, A. E. Lawrence and J. McGreevy, "Linear sigma models for open strings," arXiv:hep-th/0109069. 
[233] M. Douglas, "D-branes, Categories, and $\mathcal{N}=1$ Supersymmetry," hepth/0011017.

[234] R. Hartshorne, "Algebraic Geometry," Springer (1977).

[235] J. Distler and S. Kachru, "(0,2) Landau-Ginzburg Theory," Nucl. Phys. B413 (1993) 213, hep-th/9309110.

[236] M. Kontsevich, "Homological Algebra of Mirror Symmetry," alg-geom/9411018.

[237] P. S. Aspinwall and R. Y. Donagi, "The heterotic string, the tangent bundle, and derived categories," Adv. Theor. Math. Phys. 2, 1041 (1998); hepth/9806094.

[238] E. R. Sharpe, "D-branes, derived categories, and Grothendieck groups," Nucl. Phys. B 561, 433 (1999); hep-th/9902116.

[239] e.g. E. Witten, "On background independent open-string field theory," Phys. Rev. D46(1992) 5467, hep-th/9208027; S. Shatashvili, "Comment on the background independent open string theory," Phys. Lett. B311 (1993) 83, hep-th/9303143; S. Shatashvili, "On the problems with background independence in string theory," Algebra and Anal. v. 6 (1994) 215, hep-th/9311177; A. Gerasimov, S. Shatashvili, "On exact tachyon potential in open string field theory," JHEP 0010 (2000) 034; J. Harvey, D. Kutasov and E. Martinec, "On the relevance of tachyons," hep-th/0003101; D. Kutasov, M. Marino and G. Moore, "Some exact results on tachyon condensation in string field theory," JHEP0010(2000) 045, hep-th/0009148.

[240] K. Hori, A. Iqbal and C. Vafa, "D-Branes and mirror symmetry"; hepth/0005247.

[241] D.-E. Diaconescu and M.R. Douglas, "D-branes on stringy Calabi-Yau manifolds"; hep-th/0006224.

[242] S. Govindarajan, T. Jayaraman, and T. Sarkar, "On D-branes from gauged linear sigma models"; hep-th/0007075.

[243] P. Mayr, "Phases of supersymmetric D-branes on Kaehler manifolds and the McKay correspondence," hep-th/0010223.

[244] K. Hori, "Linear models of supersymmetric D-branes," hep-th/0012179.

[245] H. Ooguri, Y. Oz and Z. Yin, "D-branes on Calabi-Yau spaces and their mirrors," Nucl. Phys. B477 (1996) 407; hep-th/9606112.

[246] D. Bigatti, L. Susskind, "Magnetic fields, branes and noncommutative geometry," Phys. Rev. D62 (2000) 066004, hep-th/9908056.

[247] J. Distler, "Notes on $(0,2)$ superconformal field theories," Trieste HEP Cosmology 1994:0322-351, hep-th/9502012.

[248] E. Silverstein and E. Witten, "Criteria for conformal invariance of $(0,2)$ models," Nucl. Phys. 444 (1995) 161; hep-th/9503212. 
[249] J.A. Harvey and G. Moore, "On the algebras of BPS states," Comm. Math. Phys. 197 (1998) 489, hep-th/9609017.

[250] T. Banks, W. Fischler, S. H. Shenker and L. Susskind, "M theory as a matrix model: A conjecture," Phys. Rev. D 55, 5112 (1997), arXiv:hep-th/9610043.

[251] M. Douglas, "D-branes, Categories and $\mathcal{N}=1$ Supersymmetry," arXiv:hepth/0011017.

[252] A. Losev, G. W. Moore and S. L. Shatashvili, "M \& m's," Nucl. Phys. B 522, 105 (1998) [arXiv:hep-th/9707250].

[253] A. Sen, "D0 branes on $T^{n}$ and matrix theory," Adv. Theor. Math. Phys. 2, 51 (1998) [arXiv:hep-th/9709220].

[254] S. Kachru, A. Lawrence and E. Silverstein, "On the matrix description of Calabi-Yau compactifications," Phys. Rev. Lett. 80 (1998) 2996, hepth/9712223.

[255] M. R. Douglas, H. Ooguri and S. H. Shenker, "Issues in (M)atrix model compactification," Phys. Lett. B 402, 36 (1997) [arXiv:hep-th/9702203].

[256] M. R. Douglas and H. Ooguri, "Why matrix theory is hard," Phys. Lett. B 425, 71 (1998) [arXiv:hep-th/9710178].

[257] T. Asakawa, S. Sugimoto and S. Terashima, "D-branes, matrix theory and K-homology," arXiv:hep-th/0108085.

[258] F. Cachazo, B. Fiol, K. A. Intriligator, S. Katz and C. Vafa, "A geometric unification of dualities," arXiv:hep-th/0110028.

[259] B. Feng, A. Hanany and Y. H. He, "D-brane gauge theories from toric singularities and toric duality," Nucl. Phys. B 595, 165 (2001).

[260] P. Seidel and R. P. Thomas, "Braid group actions on derived categories of coherent sheaves," arXiv:math.ag/0001043.

[261] W. I. Taylor, "D-brane field theory on compact spaces," Phys. Lett. B 394, 283 (1997) [arXiv:hep-th/9611042]. 